\title{
Gas-assisted focused electron beam and ion beam processing and fabrication
}

\author{
Ivo Utke ${ }^{\text {a) }}$ \\ EMPA, Swiss Federal Institute of Materials Testing and Research, Feuerwerkerstrasse 39, CH-3602 Thun, \\ Switzerland \\ Patrik Hoffmann \\ Advanced Photonics Laboratory, Ecole Polytechnique Fédérale de Lausanne, CH-1015 Lausanne, \\ Switzerland \\ John Melngailis \\ Department of Electrical and Computer Engineering, Institute for Research in Electronics and Applied \\ Physics, University of Maryland, College Park, Maryland 20742
}

(Received 16 January 2008; accepted 3 June 2008; published 11 August 2008)

Beams of electrons and ions are now fairly routinely focused to dimensions in the nanometer range. Since the beams can be used to locally alter material at the point where they are incident on a surface, they represent direct nanofabrication tools. The authors will focus here on direct fabrication rather than lithography, which is indirect in that it uses the intermediary of resist. In the case of both ions and electrons, material addition or removal can be achieved using precursor gases. In addition ions can also alter material by sputtering (milling), by damage, or by implantation. Many material removal and deposition processes employing precursor gases have been developed for numerous practical applications, such as mask repair, circuit restructuring and repair, and sample sectioning. The authors will also discuss structures that are made for research purposes or for demonstration of the processing capabilities. In many cases the minimum dimensions at which these processes can be realized are considerably larger than the beam diameters. The atomic level mechanisms responsible for the precursor gas activation have not been studied in detail in many cases. The authors will review the state of the art and level of understanding of direct ion and electron beam fabrication and point out some of the unsolved problems. (C) 2008 American Vacuum Society.

[DOI: $10.1116 / 1.2955728$ ]

I. INTRODUCTION. . . . . . . . . . . . . . . . . . . . . . . . . 1198

A. Electron and ion beams. ............. 1199

B. Electron and ion beam properties. . . . . . . 1200

1. Electron beams. . . . . . . . . . . . . . 1200

2. Focused ion beams. .............. 1200

C. Characteristics of incident primary electron and ion beams................ 1201

II. PRINCIPLES AND FUNDAMENTALS

OF GAS-ASSISTED FEB AND FIB

DEPOSITION

AND ETCHING. . . . . . . . . . . . . . . . . . . 1202

A. Nonlocal surface effects due to electron and ion interactions. . . . . . . . . . . . . . . 1204

1. Interaction mechanisms. . . . . . . . . . . . . 1204

2. Energy spectra of emitted electrons and of activated surface atoms. . . . . . 1205

3. Radial density distribution of surface interactions. . . . . . . . . . . . . . 1206

B. Electron interaction with molecules....... 1207

1. Electron interaction with gas phase molecules. . . . . . . . . . . . . . . 1207
2. Electron interaction with adsorbed molecules. . . . . . . . . . . . . . . . . . . . . 1208

3. Electron stimulated desorption. . . . . . . . 1209

4. Physical sputtering due to electrons. .... 1210

C. Ion interaction with molecules.......... 1210

1. Ion interaction with adsorbed molecules. . 1210

2. Physical sputtering due to ions........ 1211

D. Impinging precursor flux. . . . . . . . . . . 1214

1. Molecule flux in chambers and at tube exit..................... 1214

2. Spatial distribution of molecule flux..... 1215

3. Shadow effects. . . . . . . . . . . . . 1216

4. Gas phase related processes. . . . . . . . 1216

E. Precursor migration. . . . . . . . . . . . . 1217

1. Adsorption and desorption. .......... 1217

2. Surface diffusion. . . . . . . . . . . . 1218

3. Electrostatic field mediated phenomena... 1219

F. FEB/FIB heating. . . . . . . . . . . . . . . . 1220

1. Plane bulk geometry. . . . . . . . . . . . 1220

2. Pillar geometry. ................ 1220

3. Membranes. . . . . . . . . . . . . . . 1222 
III. FEB AND FIB CONTINUUM MODELS....... 1222

A. Steady state solutions.............. 1223

B. Parameter determination from steady state exposures. . . . . . . . . . . . . . . . 1225

C. Time dependent solutions for pulsed irradiation. .................... 1226

D. Parameter determination from raster scan exposures.......................

E. Conditions for the electron- or ion-limited regime......................

F. Models accounting for several species of adsorbates....................

IV. MONTE CARLO MODELS FOR GAS ASSISTED FEB INDUCED DEPOSITION......

A. Monte Carlo models without precursor dynamics. ......................

B. Monte Carlo models with precursor dynamics................... 1231

V. PRECURSOR MOLECULES. . . . . . . . . . . . 1232

A. General aspects................... 1233

1. The role of residual molecules in microscope chambers............. 1233

2. Precursor stability............. 1233

3. Vapor pressure and evaporation enthalpy.. 1233

B. Complexes for deposition............ 1233

1. Organic compounds for $\mathrm{C}$ deposition..... 1234

2. Hydrides. . . . . . . . . . . . . . . 1236

3. Halides. . . . . . . . . . . . . . . . . . . 1237

4. Carbonyls.................. 1237

5. Pure phosphines and halogenophosphines. 1240

6. Organometallics................ 1242

7. Acetylacetonates............... 1243

8. Alkoxides, nitrates, and amides........ 1243

9. Precursors for oxide deposition (dielectrics).................... 1244

10. Postdeposition treatments........... 1245

C. Precursors and additional reactive gases.... 1247

1. Metals, metal oxides, and metal nitrides. . 1247

2. Pure silicon dioxide $\left(\mathrm{SiO}_{2}\right) \ldots \ldots \ldots \ldots 1247$

D. Etchants. . . . . . . . . . . . . . . . . . . . 1248

1. FEB gas-assisted etching. . . . . . . . . . 1248

2. FIB gas enhanced etching........... 1249

E. Nonvolatile compounds.............. 1251

VI. PROCESS CONTROL AND

CHARACTERIZATION OF DEPOSITS. ...... 1251

A. Time-resolved process control. .......... 1251

1. Reflectometry.................. 1251

2. Monitoring of sample current and secondary electron signal............ 1253

3. Mass sensing. ................ 1254

4. In situ electrical resistance measurements. 1255

5. In situ observation studies............ 1255

B. Composition and substructure......... 1256

C. SEM integrated mechanical measurements... 1257

VII. APPLICATION FIELDS IN RESEARCH

AND INDUSTRY.................... 1257

A. Repair of photomasks................. 1258
B. Scanning probe sensors............. 1258

C. Circuit editing. .................... 1259

D. Nanophotonics. . . . . . . . . . . . . . . . 1259

E. Micro- and nanoelectronics. . . . . . . . . . . 1261

1. Insulators and resistors. . . . . . . . 1261

2. Electrical contacts............... 1261

3. Laboratory prototype devices......... 1262

F. Field emitters. . . . . . . . . . . . . 1263

G. Mask fabrication for pattern transfer...... 1263

H. Mechanical applications............... 1263

I. Biorelated applications. . ............... 1264

VIII. SUMMARY AND OUTLOOK. . . . . . . . . . 1264

A. FEB versus FIB. . . . . . . . . . . . . . 1264

B. Process regimes................ 1265

C. Precursor molecules and deposit purity..... 1265

D. Fragmentation channels and reaction paths... 1266

E. Resolution. . . . . . . . . . . . . . . . 1266

F. Models...................... 1266

G. Fundamental issues................. 1267

H. Future prospects................ 1267

1. Helium ion beam.............. 1267

2. Projection maskless patterning........ 1267

APPENDIX A: RANGES OF ELECTRONS AND

IONS. . . . . . . . . . . . . . . . . . . . . . . . 1268

APPENDIX B: ELECTRON AND ION ENERGY

LOSSES AND STOPPING POWERS. . . . . . . . . . . 1269

APPENDIX C: SKIRT DISTRIBUTION. . . . . . . . 1269

APPENDIX D: DEPOSITED ENERGY......... 1269 APPENDIX E: THE DIFFUSION PARAMETER

$C_{2} \ldots \ldots \ldots \ldots \ldots \ldots \ldots \ldots \ldots \ldots \ldots \ldots$

\section{INTRODUCTION}

The impressive development in micro-/nanofabrication tools and processes over the past half century has been closely linked to the integrated circuit (IC) industry. The drive has been to economically and reliably pattern as large an area as possible with ever finer dimensions. The minimum gate lengths in the most advanced chips are approaching the $32 \mathrm{~nm}$ regime, and the semiconductor industry projects that this figure will be cut at least in half before truly fundamental limits are reached. An enormous engineering effort over the past half century has resulted in the ability to "print" complex integrated circuits with nanometer dimensions cheaply and over large areas, albeit in factories that cost $\$ 2$ billion. The patterns on the surface of a wafer are defined in resist by, usually, optical lithography and then transferred to the substrate by some material alteration or removal technique. This is called "planar processing." We will consider two "nonplanar" processes of surface alteration: focused electron beams and focused ion beams.

Nonplanar techniques of fabrication have been developed, whether for research applications, for potentially novel manufacturing, or, in special cases, as aids to the IC manufacturing process. The techniques for modifying a surface locally at micron and submicron dimensions or fabricating in

\footnotetext{
$\overline{\left.{ }^{a}\right)}$ Electronic mail: ivo.utke@empa.ch
} 


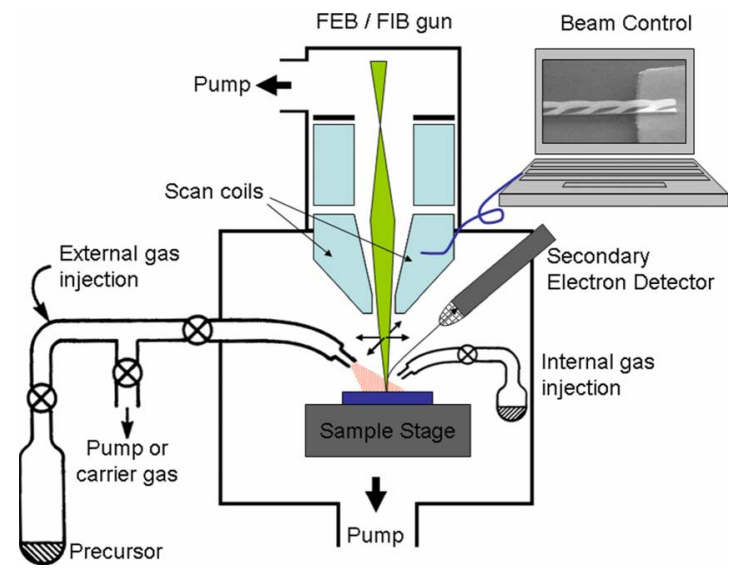

FIG. 1. Schematics of FIB and FEB nanofabrication systems. Gas injection systems (GIS) with external and internal precursor reservoirs are shown. Beam control is achieved by lithography software addressing pixels within the field of view for a defined dwell time.

three dimensions include laser beams, neutral atom beams, nanoprobes, as well as electron beams and ion beams. Nanoprobes are unique in that they have been used to move and place individual atoms as well as to locally modify a surface chemically or mechanically. On the other hand, photon, electron, and ion beams are generated remotely and impinge on the surface to deliver energy that directly removes or modifies the surface or, in the presence of an adsorbed precursor gas, induces a chemical reaction that deposits or removes material. The laser based processes were developed in the 1980s and are generally useful for patterning at dimensions above a micrometer. ${ }^{1}$

\section{A. Electron and ion beams}

Focused electron and focused ion beams can have diameters below $10 \mathrm{~nm}$ and occupy a range between individual atom manipulation with scanning tunneling microscope probes and laser beams, both in dimensions and writing speed. They also have commonalities in how they are generated and used.

The apparatus used in both cases resembles a scanning electron microscope. A column about $30-50 \mathrm{~cm}$ tall and 15-20 cm diameter contains a "point" source of electrons or ions, charged particle lenses that focus this point on the sample at some energy, and means of deflecting or blanking off the beam. The range of energies is usually between 1 and $50 \mathrm{keV}$. The column is mounted on a vacuum chamber that has an $x-y$ sample stage and in most cases has a means of introducing a precursor gas. A schematic of the configuration used for either electron or ion induced processes is shown in Fig. 1. The gas is introduced to the close proximity of where the beam is incident on the sample through a needle of $\sim 1 \mathrm{~mm}$ diameter.

Anyone using a scanning electron microscope (SEM) has probably observed that the surface becomes contaminated with a film during exposure to the beam. The film is presumed to form from hydrocarbons that are present in the vacuum system, such as pump oil, which adheres to surfaces and is altered by the electron beam. From the beginning of electron microscopy, the presence of contaminant gases and also the etching of such contamination in the presence of either water vapor or residual air had been observed. ${ }^{2-6}$ The contamination deposition phenomenon has, in fact, been exploited to write sub-100-nm features as early as $1976{ }^{7}$ Moreover, as early as 1960 gas (silicone oil vapor) was deliberately introduced into a vacuum system and bombarded with electrons to form a polymer film. ${ }^{8}$ It is surprising that only in the 1980s did researchers deliberately introduce gases other than pump oils into SEM chambers to deposit other materials. $^{9-11}$ Although in the following years numerous precursor gases were demonstrated and various structures fabricated, the practical applications of electron beam induced processing as a micro-/nanofabrication technique did not grow rapidly until recently. This is partly due to the introduction of focused ion beams that were rapidly accepted as milling tools for micro- and nanofabrication.

The usefulness of a focused beam of ions as a micronanofabrication tool was pointed out as early as $1973 .^{12,13}$ Using a conventional ion implanter as the source of ions, a $3 \mu \mathrm{m}$ diameter beam was achieved and some of the possible applications were demonstrated. Due to the limited brightness of this type of source, the current density at the sample was only about $10^{-4} \mathrm{~A} / \mathrm{cm}^{2}$ so that any processing was very slow. Although $10^{4}-10^{5}$ time brighter cryogenic sources were investigated soon thereafter, ${ }^{13,14}$ they were difficult to use and the practical focused ion beam (FIB) applications did not develop rapidly until the invention of the gallium liquid metal ion source. ${ }^{15,16}$ Resistless electron beam fabrication needs a precursor gas unless ultrahigh current densities are used, while ion beams can directly mill, implant, or damage material as well as deposit or etch in the presence of a gas ambient. Thus FIB applications were developed in the integrated circuit industry for photomask repair, fault diagnostics, circuit restructuring, and transmission electron microscopy (TEM) sample preparation. In research various other applications have been demonstrated, such as device fabrication by direct, maskless implantation, local damage, and high resolution secondary ion mass spectrometry (SIMS).

Recently electron beam direct fabrication has attracted increasing attention because of the unavoidable ion implantation and surface damage that accompanies any application of FIBs. In photomask repair, for example, $\mathrm{Ga}^{+}$ions are implanted when chromium or other masking material is milled off. This reduces the optical transmission through the underlying quartz. As the wavelengths of the exposing radiation have decreased to $193 \mathrm{~nm}$, and as the exposure tolerances have become more stringent, this loss of transmission is no longer acceptable. Consequently, electron beam induced etching and deposition using a precursor gas are being developed for mask repair. ${ }^{17,18}$ For other applications, such as circuit restructuring and fault diagnostics, FIB fabrication still plays an important role.

The review literature published so far comprises one review ${ }^{19}$ and one book ${ }^{20}$ on gas assisted electron beam nanofabrication and Monte Carlo (MC) simulations that were 
TABLE I. Properties of electron beam systems. The numbers quoted are very approximate. The minimum beam diameter in any system in practice depends also on electronic noise and vibration. As the beam acceptance angle is increased, the beam current and the beam diameter increase but over much of the range the current density incident on the sample remains approximately constant. Also as the beam energy is lowered, the beam diameter increases.

\begin{tabular}{|c|c|c|c|c|}
\hline & $\begin{array}{l}\text { Energy } \\
\text { of beam } \\
(\mathrm{keV})\end{array}$ & $\begin{array}{c}\text { Minimum } \\
\text { beam } \\
\text { diameter }(\mathrm{nm})\end{array}$ & $\begin{array}{l}\text { Current } \\
\text { density } \\
\left(\mathrm{A} / \mathrm{cm}^{2}\right)\end{array}$ & Remarks \\
\hline $\begin{array}{l}\text { SEM-field emission } \\
\text { electron source }\end{array}$ & $1-30$ & $1-10$ & 2000 & $\begin{array}{l}\text { Beam steering and blanking needs } \\
\text { to be added for all SEMs. }\end{array}$ \\
\hline $\mathrm{SEM}-\mathrm{LaB}_{6}$ source & $1-30$ & $3-10$ & 20 & $\begin{array}{l}\text { Processing results can be imaged } \\
\text { immediately. Stage tilt available. }\end{array}$ \\
\hline SEM-W source & $1-30$ & $5-10$ & 2 & \\
\hline E-beam lithography & $20-100$ & $5-10$ & 1000 & $\begin{array}{l}\text { No stage tilt. Sophisticated beam } \\
\text { placement and blanking. Planar } \\
\text { imaging. }\end{array}$ \\
\hline STEM & $200+$ & 0.2 & $\sim 10^{5}$ & Restricted sample insertion. \\
\hline
\end{tabular}

published recently. A number of recent reviews exist for ion beam nanofabrication ${ }^{21-32}$ and two books; ${ }^{33,34}$ however, they treat mostly the fundamentals and applications of physical sputtering (milling) and review gas-assisted processes at a technological level or just very briefly. ${ }^{35}$ The comparison of gas-assisted ion and electron beam processing also at a technological level was given in Ref. 36. Our review is different in that we focus on the fundamental aspects of gas-assisted material removal and addition in electron and ion beam processes. Because the applications of both beams often aim at similar goals and because a number of the fundamental aspects are similar, we treat both beams together.

\section{B. Electron and ion beam properties}

The sophisticated charged particle generation and manipulation apparatus in the electron and ion columns has been developed over many decades. We will not review this development here but rather concentrate on the properties of the beams incident on the samples, such as beam diameter, beam manipulation, current density, and energy. These parameters play a key role in the material removal, deposition, and alteration processes, which are our main focus.

\section{Electron beams}

The systems used to produce an incident electron beam of nanometer dimensions on a surface can be considered in three categories: SEMs, electron beam lithography systems, and scanning transmission electron microscopes (STEMs).

SEM's and electron beam lithography systems are in principle similar but have some different performance requirements. Both strive for minimum beam diameters below $10 \mathrm{~nm}$ and maximum current density in the beam. For lithography the beam energy is normally in the $20-100 \mathrm{keV}$ range and beam stability or beam placement accuracy on the sample is of paramount importance. Genuine e-beam lithography systems including precision stages have not been, but certainly could be, used for the kind of processing we are considering. They are too expensive and are usually dedicated to only resist exposure. For SEMs the beam energy is usually in the $1-30 \mathrm{keV}$ range and the minimum beam di- ameter is near $1 \mathrm{~nm}$. For the fabrication techniques of interest here, modified SEMs have been used because they are simpler, cheaper, and have easily accessible vacuum chambers. The modification needed is beam placement and scanning control that resembles e-beam lithography but is extended to cope with other exposure and blank strategies. STEMs operate at energies in hundreds of keV. The sample placement is usually quite restrictive since it has to be inside an electron lens. The properties of electron beam systems important to beam induced processing are summarized in Table I.

\section{Focused ion beams}

The FIB apparatus externally is very similar to the electron beam apparatus. In fact there are dual beam systems that have both an electron beam column and an ion beam column mounted on the same chamber.

A focused ion beam system can be regarded as composed of three parts: the source, the ion optical column, and the stage and beam control. The design and performance of these systems has been treated extensively elsewhere. ${ }^{34}$

The ion source most often used today is the liquid metal ion source. It consists of a reservoir of liquid metal, which feeds the liquid metal to a sharpened needle, usually tungsten. This source has a high brightness, typically $\sim 10^{6} \mathrm{~A} / \mathrm{cm}^{2} \mathrm{sr}$. The ion species used almost exclusively in the applications we are considering here is $\mathrm{Ga}^{+}$. Since the gallium ion has effects on the processing other than just energy delivery to the surface (damage, amorphization, and implantation), we need to mention other ion species that may turn out to be important in future ion beam processing.

Alloys can also be used, for example, $\mathrm{Au} / \mathrm{Si}, \mathrm{Au} / \mathrm{Si} / \mathrm{Be}$, or $\mathrm{Pd} / \mathrm{As} / \mathrm{B}$. The liquid alloy has to have a low enough vapor pressure to operate in vacuum, and not react with, yet wet, the needle. For the alloy a crossed-electric and magnetic-field filter is used in the column to select the desired ion species. Other species not available in liquid metal form, such as hydrogen or noble elements, may be desirable in many cases since they would not contaminate the sample the way Ga or other metal ions would. For these species the 
TABLE II. Comparison of various ion sources. Unnormalized brightness is most often quoted in the literature. To get normalized (reduced) brightness divide by the extraction voltage typically about $10 \mathrm{kV}$. The total beam current and the beam diameter (usually taken as FWHM see below) depend on the acceptance angle, but the current density remains constant over much of the range.

\begin{tabular}{|c|c|c|c|c|c|}
\hline $\begin{array}{l}\text { Type of } \\
\text { ion source }\end{array}$ & $\begin{array}{l}\text { Ion } \\
\text { species }\end{array}$ & $\begin{array}{c}\text { Unnormalized } \\
\text { brightness }\left(\mathrm{A} / \mathrm{cm}^{2} \mathrm{sr}\right)\end{array}$ & $\begin{array}{l}\text { Current density } \\
\text { on sample } \\
\left(\mathrm{A} / \mathrm{cm}^{2}\right)\end{array}$ & $\begin{array}{l}\text { Minimum beam } \\
\text { diameter }(\mathrm{nm}) \\
(\text { at } 30 \mathrm{kV})\end{array}$ & Ref. \\
\hline Liquid metal & $\mathrm{Ga}^{+}$ & $3 \times 10^{6}$ & 10 & 10 & 15 and 16 \\
\hline $\begin{array}{l}\text { Liquid } \\
\text { metal alloy }^{\mathrm{a}}\end{array}$ & $\begin{array}{c}\mathrm{Au} / \mathrm{Si} / \\
\mathrm{Be}\end{array}$ & $\begin{array}{l}\sim 10^{5} \text { depends on } \\
\text { the } \% \text { of the desired } \\
\text { species in beam }\end{array}$ & $0.1-1.0$ & 50 & 37 \\
\hline Gas field ion (supertip) & $\mathrm{He}^{+}$ & $5 \times 10^{9}$ & 1000 & 5 & 38 and 39 \\
\hline Gas field ion source & $\mathrm{He}^{+}$ & $4 \times 10^{9}$ & $\cdots$ & 0.6 & 40 and 41 \\
\hline Multicusp plasma & $\mathrm{Kr}^{+}$ & $0.55 \times 10^{3}$ & $1.2 \times 10^{-2}$ & 100 & 42 \\
\hline
\end{tabular}

${ }^{\mathrm{a}}$ For a table of other alloys and references, see Ref. 32.

gas field ion source and the gaseous plasma source have been considered. The former, although brighter than the liquid metal, has to operate at cryogenic temperatures and is hard to use. The plasma source is orders of magnitude less bright than the liquid metal but relatively robust and easy to use. The plasma source has the advantage that many species of ions can be used and, in special circumstances, for example, when an intense beam is needed at larger dimensions, it may outperform the liquid metal ion source in spite of the limited brightness. The sources and their properties are summarized in Table II.

The details of the surface alteration effects that a beam of electrons or ions produces are determined by the spatial dependence of the current density (or particle flux) in the beam. The most important factors determining the current density distribution are the source of the particle current (ideally a point source) and the charged particle optical column, which ideally focuses this point source on the sample.

\section{Characteristics of incident primary electron and ion beams}

According to Fig. 2, for an astigmatism and aberration- free incident beam, the central part of the radial flux distribution incident on a plane surface can be well described by a Gaussian,

$$
f(r)=\frac{\left(I_{P} / e_{0}\right)}{2 \pi a^{2}} \exp \left(-\frac{r^{2}}{2 a^{2}}\right)
$$

where $f(r)$ is in electrons or ions per unit area and time, $a$ is the standard deviation, $I_{P}$ is the beam current, and $e_{0}$ is the elementary charge. $I_{P}$ can be measured in a Faraday cage. The standard deviation can be derived from knife edge measurements, ${ }^{43,44}$ resolution measurements of $\mathrm{Au}$ spheres on $\mathrm{C}^{45}$ or from the frequency spectrum of specifically defined patterns. ${ }^{46,47}$ The latter method and the knife edge method have the advantage of being an operator independent measurement. The frequency spectrum of images with nonspecific patterns is also used but the relation of such determined beam size to the standard deviation depends on the (individual) signal to noise cutoff. ${ }^{48}$ Different definitions of beam size are in use. For any incident peak function, one can define the widths at $1 / e, 1 / e^{2}(e=$ Euler's constant), the full width at half maximum (FWHM), and full widths (FW) comprising $50 \%$ or $90 \%$ of all electrons or ions. For the
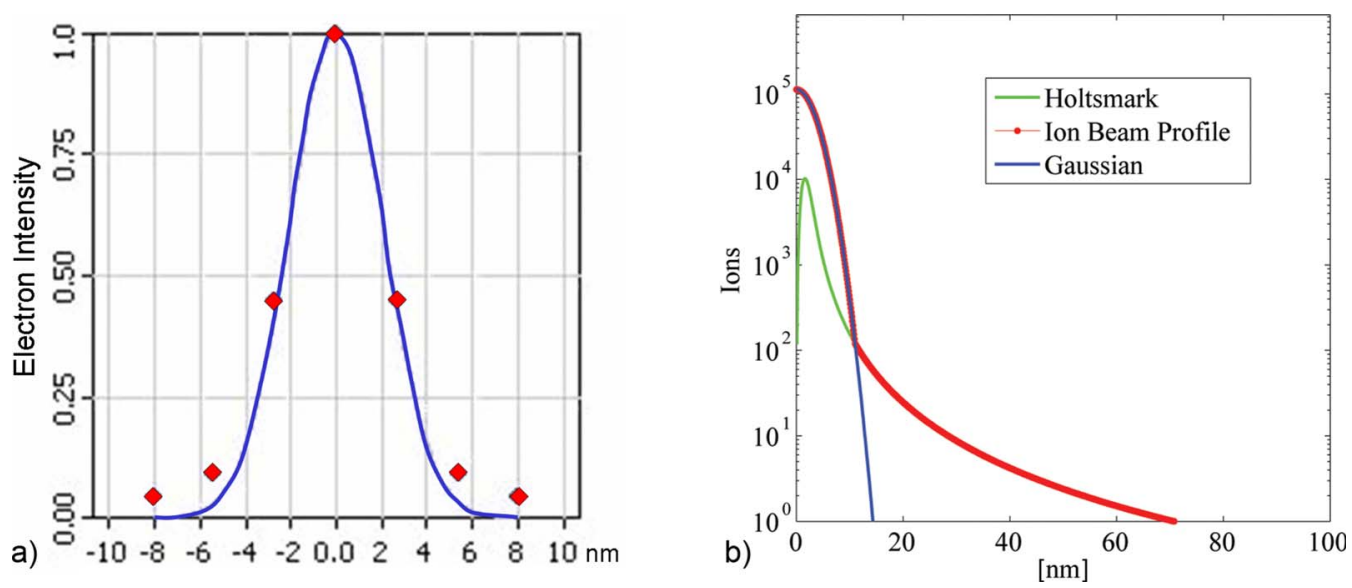

FIG. 2. Distribution of focused electron and ion beams. (a) Measured FEB distribution ( $20 \mathrm{keV}$ ): the solid line is a Gaussian with FWHM=4.4 nm. Courtesy of S. Babin, Abeam Technologies. (b) Simulated Holtsmark dìstribution for $30 \mathrm{keV} \mathrm{Ga}^{+}$ions at $1 \mathrm{pA}$. The FIB has a FWHM=7 nm but extends to $70 \mathrm{~nm}$. Based on Ref. 52, courtesy V. Callegari, Empa. 

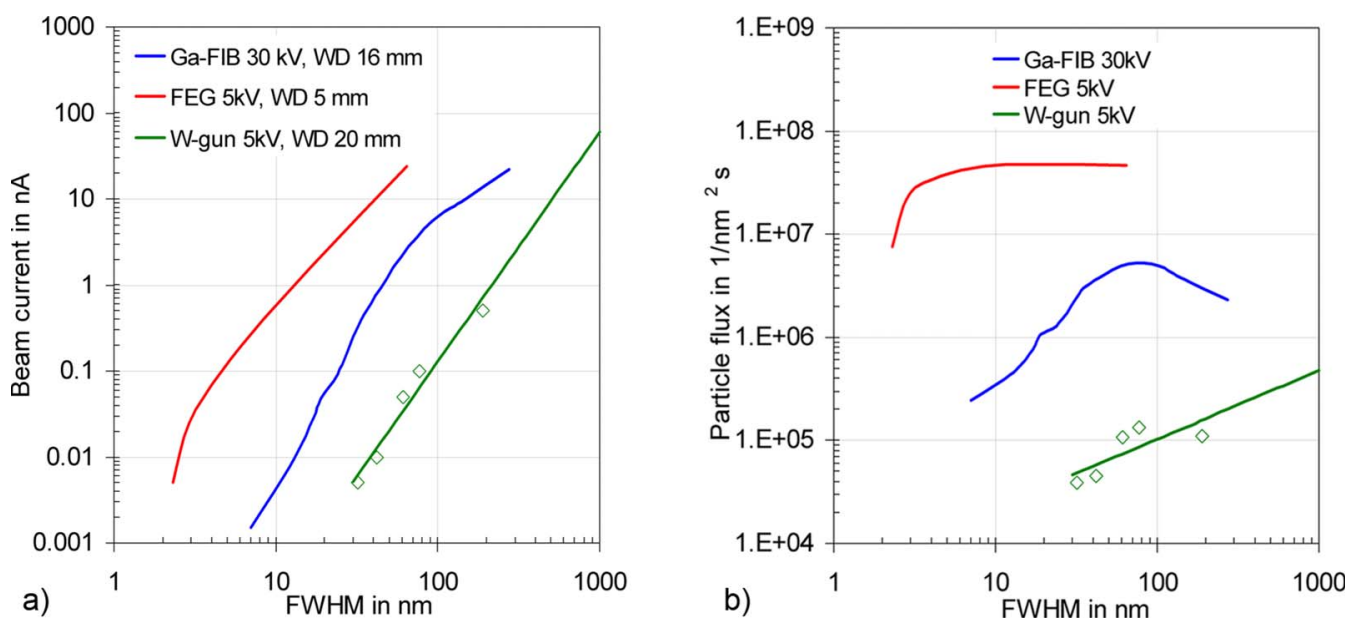

FIG. 3. Beam size of the FEI Novalab Dual Beam with a Schottky field emission gun (FEG) and a liquid Ga metal ion source (Ref. 54) and a Hitachi S3600 with a thermionic W filament. WD stands for working distance. (a) Maximum beam current vs beam FWHM. Symbols represent measured values (from frequency spectrum) extrapolated with a $I_{p}=d^{8 / 3}$ dependence. (b) Corresponding average incident particle flux.

Gaussian distribution [Eq. (1.1)] the widths become $W_{1 / e}$ $=2 a \sqrt{2}, \quad W_{1 / e^{2}}=4 a, \quad \mathrm{FWHM}=2 a(2 \ln 2)^{1 / 2} \approx 2.355 a$, FW50 $=\mathrm{FWHM}$, and $\mathrm{FW} 90=2 a(2 \ln 10)^{1 / 2} \approx 4.292 a$. The long low-intensity tails of the radial ion beam distribution ${ }^{49,50}$ [see Fig. 2(b)] can be modeled with a Holtsmark distribution arising from the Coulomb repulsion between charged particles. ${ }^{51}$ The FIB tails are responsible for the halo deposition and halo etching (overspray) around exposed areas and may affect the fabrication of closely spaced structures.

The main characteristics of charged particle beams are the acceleration (or landing) voltage, the current, and the beam diameter impinging on the substrate. The smallest beam diameter is obtained for the highest energy and smallest working distance. However, in typical particle beam machining setups, the working distance is larger to provide space for the precursor supply and thus not minimized to obtain the best resolution. Furthermore, the beam energy of choice corresponds to the best deposition or etching results, which is not necessarily the highest energy available from the optical columns. Thus the beam profile corresponding to the experiment should be measured with the above mentioned methods. Calculated examples for the influence of the beam acceleration voltage on beam diameter can be found in Refs. 34 and 53.

Figure 3(a) shows beam current versus beam diameter curves for a state of the art dual beam machine with field emission gun and liquid metal Ga ion source and for a tungsten filament SEM. Average fluxes of primary electrons or ions were calculated as $f=\left(I_{p} / e_{0}\right) /\left(\pi d^{2} / 4\right), d=\mathrm{FWHM}$, and are presented in Fig. 3(b). A typical range of $f_{0}=\left(10^{4}-5\right.$ $\left.\times 10^{7}\right) \mathrm{nm}^{-2} \mathrm{~s}^{-1}$ can be identified for scanning electron and scanning ion microscopes. The best FWHM for Ga-FIB is reported to be $5 \mathrm{~nm}$ at $35 \mathrm{keV}$ and $2 \mathrm{pA} .{ }^{55}$ For focused electron beam (FEB) the FWHM for $>100 \mathrm{keV}$ high-energy beams, usually used in STEMs, can be smaller than $1 \mathrm{~nm}$. The beam current defines the average time between the arrivals of two subsequent incident particles $\left(e_{0} / I_{p}\right)$; for $1 \mathrm{nA}$ it is about $0.2 \mathrm{~ns}$. The average time between particle arrivals on the surface may be important for both electrons and ions. If the surface activation produced by one particle has not decayed to equilibrium before the next particle arrives, one will likely observe dose rate effects.

\section{PRINCIPLES AND FUNDAMENTALS OF GAS-ASSISTED FEB AND FIB DEPOSITION AND ETCHING}

Conceptually, etching and deposition induced by FIB and FEB is considered as a reaction with surface adsorbed molecules having second order kinetics. Hence the dissociation rate is proportional to the surface density of both molecules and electrons or ions, see Fig. 4. The proportionality implies that any other possible elementary intermediate reactions leading to the final dissociation product occur on a time scale that is fast compared to the time of two successive charged particle impacts.

In a system with rotational symmetry, the vertical FEB deposition or etch rate $R(r)$ (in units of dimension per unit time) as a function of the distance $r$ from the center of the primary electron (PE) beam is for steady state, ${ }^{56}$

$$
R(r)=V n(r) \int_{0}^{E_{0}} \sigma(E) f(r, E) d E,
$$

where $V$ is the volume of the decomposed molecule or etched atom, $n(r)$ is the number of adsorbed molecules per surface unit, $\sigma(E)$ is the energy dependent electron impact dissociation cross section (leading to deposition of nonvolatile fragments or to volatile etch products depending on the molecule), and $E_{0}$ is the energy of the incident PEs. The flux distribution $f(E, r)$ is a convolution of the incident primary beam with the emitted secondary and backscattered electrons and their respective energy spectrum.

Similar considerations apply for FIB where the spatial distribution being responsible for molecule dissociation is a convolution of the primary beam distribution with the distribution of excited surface atoms generated by the collision 

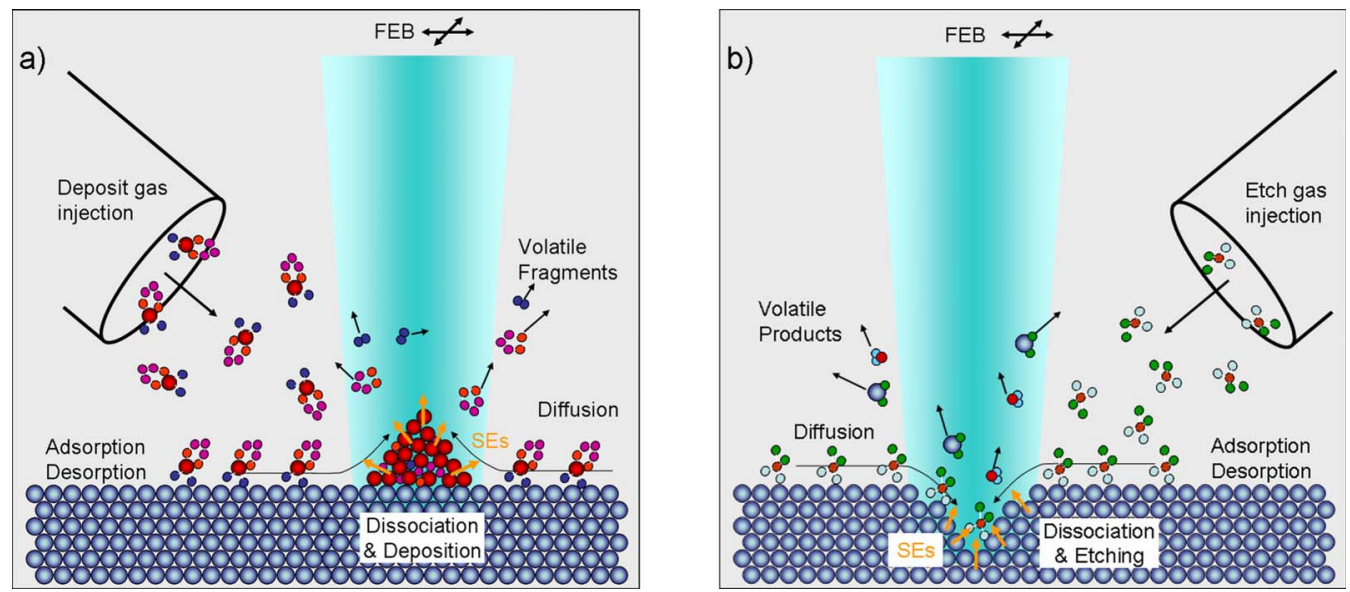

FIG. 4. Precursor depletion and replenishment in FEB induced processing: Molecules adsorb, desorb, and diffuse at the surface and are dissociated under electron impact. (a) FEB induced deposition: the nonvolatile dissociation products form the deposit growing coaxially into the beam. Volatile fragments are pumped away. (b) FEB induced etching: the surface adsorbed molecules dissociate under electron impact into reactive species and react to volatile compounds with the substrate material.

cascade $^{57}$ (see Sec. II A 1. As shown in Fig. 5, for the FIB deposition and etching rate an additional physical sputter term must be taken into account. Frequently the "FIB" notation of Eq. (2.1a) is in terms of yields,

$$
Y_{\text {net }}=Y_{\text {ch }} \pm Y_{S} \text {, }
$$

where $Y_{\text {net }}$ is the net deposition or etch yield, $Y_{\text {ch }}$ is the chemical deposition or etch yield due to dissociation of the molecule, and $Y_{S}$ is the physical sputter yield. The plus sign applies for gas-assisted FIB etching, whereas the minus sign holds for gas-assisted FIB deposition. The dependence on energy and radius is not explicitly noted anymore since the yields are given in units of dissociated or sputtered atoms per incident ions. The conversion of the sputter yield into a sput-

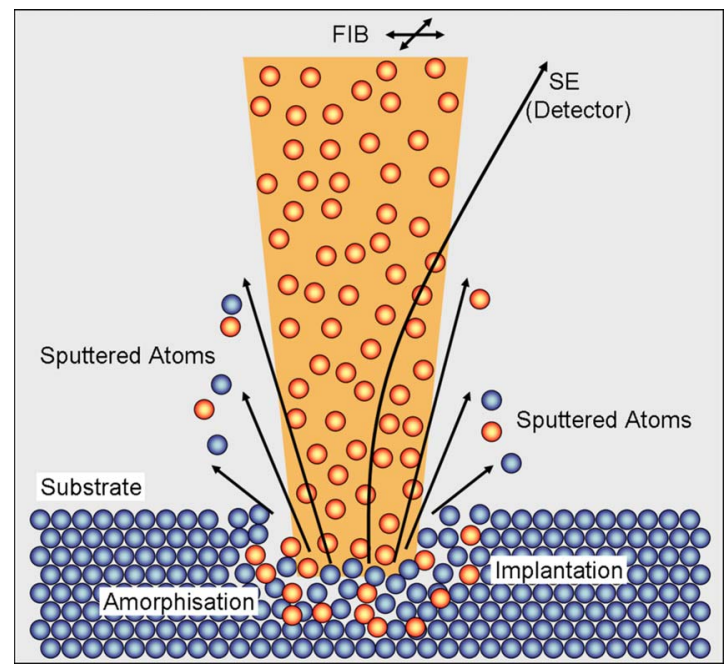

FIG. 5. Principle of FIB milling (or sputtering). Note the amorphized surface region containing implanted ions. This physical sputter contribution is also present during gas-assisted FIB etching and deposition, which was omitted here for clarity. For electrons physical sputtering is orders of magnitudes lower at the same charged particle energy due to the different masses of electrons and ions. ter rate $R_{S}$ is differently formulated in literature for FIB. In the case of FIB induced deposition, the physical sputter rate is assumed to be independent of the number of adsorbed precursor molecules ${ }^{58}$ and $R_{S}(r)=Y_{S} V f(r)$. In the case of gas enhanced etching, the physical sputter rate becomes adsorbate dependent and is inversely proportional to the adsorbate coverage ${ }^{59} R_{S}(r)=Y_{S} V f(r)\left(1-n(r) / n_{0}\right)$, where $n_{0}$ is the density of a complete monolayer. Conversion of the chemical deposition or etch yield into a chemical deposition or etch rate yields $R_{\mathrm{ch}}(r)=Y_{\mathrm{ch}} V f(r) \cdot n(r) / n_{0}$, i.e., the chemical rate is proportional to the adsorbate coverage. Using the relation $\sigma=Y_{\mathrm{ch}} / n_{0}$, the FEB notation $R_{\mathrm{ch}}(r)=\sigma V f(r) \cdot n(r)$ of Eq. (2.1a) is obtained (without explicit notation of energy). Of note is that the chemical reaction yield for deposition must override the physical sputter yield in order to get a (visible) net deposit. Otherwise material removal (sputtering) will occur.

The next conceptual point is a differential adsorption rate equation describing the behavior and surface density of molecules. Four key processes as shown in Fig. 4 are generally considered to determine the surface density $n(r, t)$ of adsorbed molecules: (a) adsorption from the gas phase governed by the precursor flux $J$, the sticking probability $s$, and coverage $n / n_{0}$; (b) surface diffusion from the surrounding area to the irradiated area governed by the diffusion coefficient $D$ and the concentration gradient; (c) spontaneous thermal desorption of physisorbed molecules after a residence time $\tau$; and (d) molecule dissociation governed by the product $\sigma f(r)$. For the molecule adsorption rate, $d n / d t$ follows

$$
\frac{\partial n}{\partial t}=\underbrace{s J\left(1-\frac{n}{n_{0}}\right)}_{\text {adsorption }}+\underbrace{D\left(\frac{\partial^{2} n}{\partial r^{2}}+\frac{1}{r} \frac{\partial n}{\partial r}\right)}_{\text {diffusion }}-\underbrace{\frac{n}{\tau}}_{\text {desorption }}-\underbrace{\sigma f n}_{\text {decomposition }} .
$$

The adsorption term in Eq. (2.2) describes a nondissociative Langmuir adsorption, where $n_{0}$ is the maximum monolayer 

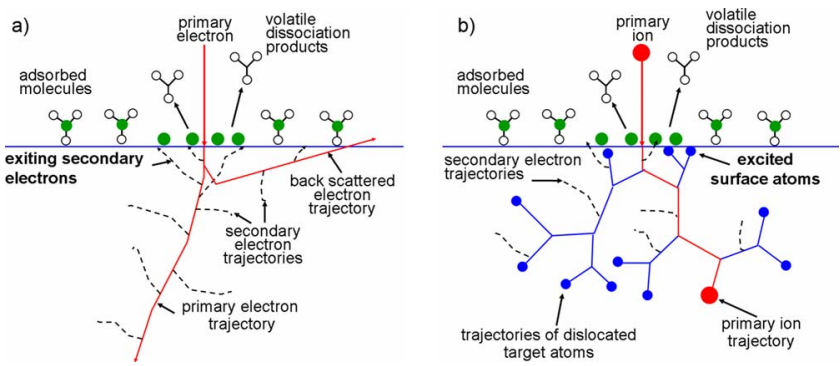

FIG. 6. (a) Scheme of interactions of primary electrons generating an emitted flux of secondary electrons and backscattered electrons. All electrons can dissociate surface adsorbed molecules via electronic excitation. (b) Scheme of interactions of primary ions generating secondary electrons and a collision cascade of substrate atoms. The nonsputtered target atoms remain as excited surface atoms contributing to molecule dissociation (modified from Ref. 57).

density given by the inverse of the molecule size. This adsorption type accounts for surface sites already occupied by nondissociated precursor molecules and limits the coverage to $n_{0}$. The parameters $n=n(r, t)$ and $f=f(r, t)$ are considered time and position dependent.

Solving Eqs. (2.1a), (2.1b), and (2.2) during FEB or FIB processing would allow predicting the shape evolution of deposits and etch holes or, in other words, spatial deposition and etch rates. The above sketched concept does not include irradiation processes in the bulk of deposits due to penetrating electrons nor any gas phase reactions. In this section we consider in detail the parameters and processes involved in Eqs. (2.1a), (2.1b), and (2.2) before we discuss their analytical and numerical solutions in Secs. III and IV.

\section{A. Nonlocal surface effects due to electron and ion interactions}

Besides the direct interaction with the beam, the dissociation of surface adsorbed molecules involves substrate medi- ated effects generated by the interaction with the incident beam. These nonlocal effects for FEB are due to the secondary and backscattered electrons, whereas for FIB the effects are due to excited surface atoms (see Fig. 6).

\section{Interaction mechanisms}

Secondary electrons (SEs) are produced between inelastic collisions of weakly bound outer shell valence electrons of semiconductors and insulators or weakly bound conduction band electrons of metals with the PEs. They are generated along the entire trajectory path of the primaries. However, only a few of these created secondary electrons can escape the substrate surface. They have exit energies situated around a peak of a few eV [see Fig. 7(a)]. The secondary electron escape depth depends on its initial energy transferred in an inelastic collision, the substrate material (composition), and the work function. It is in the order of a few nanometers. Backscattered electrons (BSEs) are reflected primary electrons due to elastic collisions having energy peaks situated near the primary electron energy and "escape" from a much larger range depending on energy given in Appendix A.

Incident primary ions also incur inelastic and elastic collisions, which are often referred to as electronic and nuclear collisions in the FIB community. Heavier ions such as gallium lose a significant fraction of their energy in nuclear collisions. As a consequence they have a very short range compared to electrons (see Appendix A) and dislocate many substrate atoms along their trajectory. The dislocated atoms themselves further collide with neighboring substrate atoms in nuclear collisions and initiate collision cascades giving rise to sputtering and a distribution of excited surface atoms around the incident primary ion beam [see Fig. 6(b)]. Secondary electrons are generated during inelastic collisions of primary ions. For $\mathrm{Ga}$ ions impinging with $1-30 \mathrm{keV}$ onto
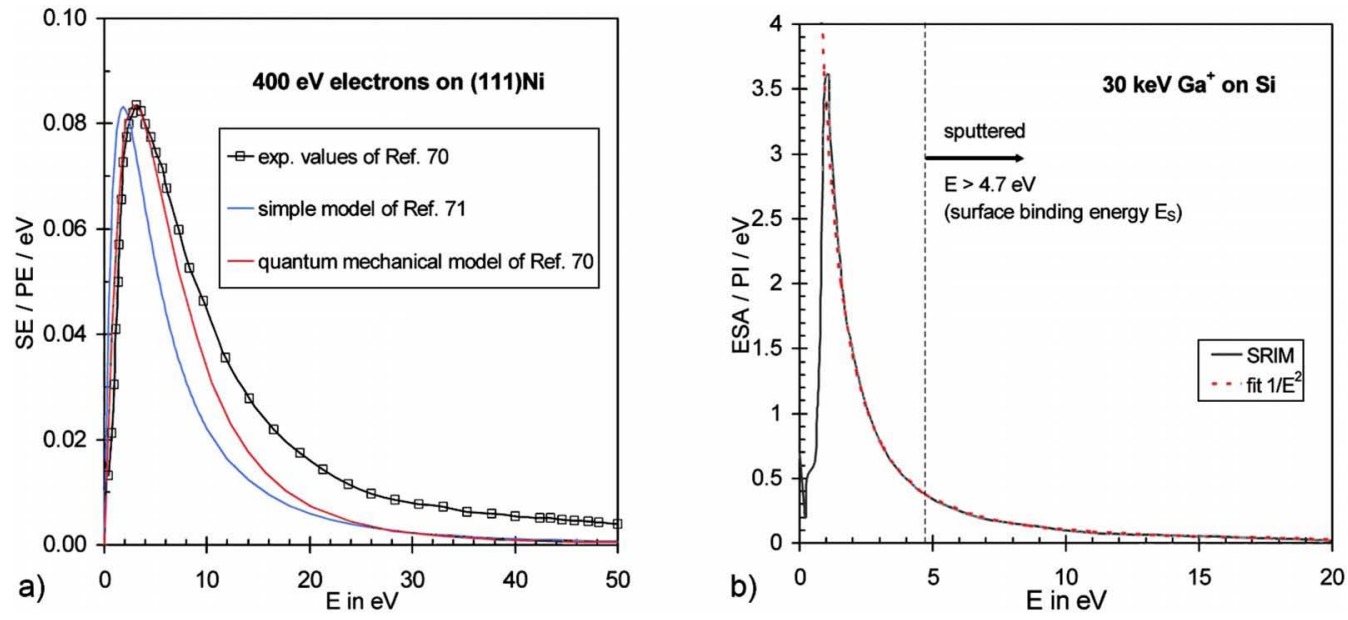

FIG. 7. Secondary electron (SE) energy spectrum per primary electron (PE). Comparison of experimental data of Ni (Ref. 70) with the quantum mechanical electron reflection model of Schaefer and Hoelzl (Ref. 70) and the model of Chung and Everhart (Ref. 71). The surface below the experimental curve was set to the secondary electron yield $=1.1$ of $\mathrm{Ni}$ at $E_{0}=400 \mathrm{eV}$. (b) Energy spectrum of excited surface atoms (ESA) per primary ion (PI). Monte Carlo simulation [SRIM (Ref. 73)] of the energy normal to the surface. The nonsputtered dislocated Si atoms constitute the excited surface atoms responsible for dissociation (surface below the curve, $E<4.7 \mathrm{eV}$ ). At $30 \mathrm{keV}$, the ESA yield is $Y_{\mathrm{ESA}}=5.5 \mathrm{Si} / \mathrm{Ga}$ ion and the sputter yield is $Y_{S}=2.6 \mathrm{Si} / \mathrm{Ga}$ ion. 
silicon, the electronic loss is about one order of magnitude smaller than the nuclear loss, whereas for He ions it is reverse (see Appendix B).

For gas-assisted FEB processing, it is still an open debate to which electrons the dissociation can be attributed. In certain experiments it is mostly attributed to the secondary electrons. ${ }^{60-65}$ However, also the primary electrons are reported in some cases to dominate or to contribute to the process. ${ }^{62,66}$ In our opinion, the dominance of secondary or primary electron interaction with molecules or, in other words, the dominance of the high-energy part of the spectrum against the low energy part of the spectrum in dissociating molecules must be verified from case to case.

For gas-assisted FIB processing, it was shown experimentally that the chemical deposition yield is linearly proportional to the number of surface atoms excited by the collision cascades. ${ }^{57,67}$ A proportionality of the secondary electron yield with the chemical deposition yield was also experimentally observed for FIB platinum deposition. ${ }^{60}$ However, an analysis of the data indicates that this mechanism is unlikely. To attribute magnitude of the dissociation yield to the secondary electrons emitted due to the ion incidence reported in this work, the yield per secondary electron from ions would have to be 23 times higher than the yield for secondary electrons emitted due to electron bombardment. Moreover, data available on the secondary electron yield of metals due to noble gas ion bombardment show that the secondary electron yield decreases with increasing ion mass. ${ }^{68}$ For example, in the case of silver, the secondary electron yield for $10 \mathrm{keV}$ xenon ions is about four to five times lower (depending on crystalline orientation) than for $10 \mathrm{keV}$ neon ions. The situation is similar in most metals. Nowhere in the tabulated cases does a secondary electron yield an increase going from neon to xenon. However, the dissociation yield for gold deposition has been shown to increase significantly in going from neon to xenon in the range of $2-10 \mathrm{keV} .{ }^{57}$

There is evidence that the mechanism of gas enhanced FIB etching is similar to the mechanism for FIB induced deposition. Measurements of the etch rate of $\mathrm{SiO}_{2}$ by all of the noble gas ions and $\mathrm{XeF}_{2}$ appear to correlate with the stopping power and the ion mass. ${ }^{69}$ Since both sputter rate and ion induced deposition rate also correlate with the stopping power, ${ }^{57}$ the gas-assisted ion etching mechanism is also likely to be explained by the same collision cascade model.

\section{Energy spectra of emitted electrons and of activated surface atoms}

Electrons incident on a substrate generate secondary electrons. An experimental secondary electron energy spectrum generated by $400 \mathrm{eV}$ primary electrons incident on $\mathrm{Ni}^{70}$ is shown in Fig. 7(a). The energy spectrum is characterized by its peak energy and FWHM. Experimentally, for clean metal surfaces the peak energy was shown to vary between 1 and $5 \mathrm{eV}$ and the energy FWHM within $3-15 \mathrm{eV} .^{70}$ A highly idealized model for the secondary electron energy distribution of Ref. 71 gives the simple relation $f_{\mathrm{SE}}(E) \sim E /(E$ $+\Phi)^{4}$, where $E$ is the energy of the secondary electrons and
$\Phi$ is the work function of the substrate. The $1 / E^{3}$ decay for higher energies fits the data well but a large mismatch with the experimental peak position is found. Better fits to experiments are obtained from the solution of the Boltzmann equation and the consideration of electron reflection ${ }^{70}$ [see Fig. $7(\mathrm{a})]$. The energy dependence of the secondary electron spectrum is independent of the PE energy for energies $>100 \mathrm{eV},{ }^{71,72}$ but the secondary electron yield changes. The secondary electron yield $Y_{\mathrm{SE}}$, in secondary electrons per primary electron [or often $\delta(\%)$ in "SEM" literature], is defined as the integral of the curve in Fig. 7(a) within the energy range $0 \leqslant E \leqslant 50 \mathrm{eV}$. The integral for $50 \mathrm{eV} \leqslant E \leqslant E_{0}$ (not shown in fig. 7(a).) gives the backscattered electron yield $Y_{\mathrm{BSE}}$ [often $\eta(\%)$ in "SEM" literature].

For electron impingement, the secondary electron yield versus incident energy follows a universal curve ${ }^{74}$ according to which the normalized yield has its maximum for most materials around $1 \pm 0.5 \mathrm{keV}$. This curve is frequently used to scale secondary electron yields in Monte Carlo simulations. However, it must be critically noted that agreement with secondary electron yields does not mean that the spectrum is correctly simulated. In fact, it strongly depends on the generation mechanism ${ }^{45,75}$ varying already for different pure bulk metals (inner shell excitation, plasmon losses, and conduction electron excitation). For typical FEB and FIB fabricated compound compositions and composite substructures like in Figs. 43 and 44, the status quo of the secondary electron generation and thus its energy spectrum is unknown. Furthermore, electrical charging can also alter the secondary electron spectrum drastically and suppress the low energy part. ${ }^{76,77}$ We stress these points here as it implies limits on the reliability of Monte Carlo (MC) simulations discussed in Sec. IV.

Secondary electron yields for electron irradiation increase with atomic number of the target material. For yields of secondary and back scattered electrons with specific targets and e-beam energies, we refer the reader to a comprehensive database. ${ }^{78}$ For in situ determinations of both yields, see Ref. 79.

Ions incident on a substrate generate (among others) excited surface atoms. The spectrum of excited surface atoms is characterized by an energy peak situated at a few $\mathrm{eV}$ and a $1 / E^{2}$ decay $^{57}$ with increasing energy [see Fig. 7(b)]. There are no experimental values available, which allow verifying the MC simulations. Excited surface atoms with energies higher than the surface binding energy $E_{S}$ (approximately the heat of sublimation) can leave the substrate and are thus sputtered. The area below the curve in Fig. 7(b) for $E>E_{S}$ $=4.7 \mathrm{eV}$ is equal to the sputter yield $Y_{S}$, and the area for $E$ $<E_{S}$ gives the yield of excited surface atoms $Y_{\mathrm{ESA}}$. Whereas the yields change with primary ion energy, the energy spectrum does not change considerably with incident energy according to SRIM simulations. ${ }^{73,80}$ In addition to excited surface atoms, secondary electrons are also produced by incident ions, which are used for imaging the samples. All yields are summarized for two incident energies on $\mathrm{Si}$ and compared for Ga ions and electrons in Table III. 
TABLE III. Comparison of yields for electron and ion irradiation of silicon with 5 and $30 \mathrm{keV}$ incident energies. Yields are in units per primary ion or per primary electron. $Y_{\mathrm{ESA}}=$ yield of excited surface atoms, $Y_{S}$ = physical sputter yield, $Y_{\mathrm{SE}}=$ secondary electron yield, and $Y_{\mathrm{BSE}}=$ backscattered electron yield.

\begin{tabular}{lccllc}
\hline \hline & $E_{0}(\mathrm{keV})$ & $Y_{\mathrm{ESA}}{ }^{\mathrm{a}}$ & \multicolumn{1}{c}{$Y_{S}{ }^{\mathrm{a}}$} & \multicolumn{1}{c}{$Y_{\mathrm{SE}}$} & $Y_{\mathrm{BSE}}$ \\
\hline $\mathrm{Ga}^{+}$ & 30 & 5.5 & 2.6 & $3.3^{\mathrm{b}}$ & $\ldots$ \\
$e^{-}$ & 30 & $\cdots$ & 0 & $0.06-0.19^{\mathrm{c}}$ & $0.15-0.18^{\mathrm{c}}$ \\
$\mathrm{Ga}^{+}$ & 5 & 4.3 & 1.7 & $2.5^{\mathrm{b}}$ & $\ldots$ \\
$e^{-}$ & 5 & $\cdots$ & 0 & $0.18-0.54^{\mathrm{c}}$ & $0.09-0.19^{\mathrm{c}}$ \\
\hline \hline
\end{tabular}

${ }^{\mathrm{a}}$ Calculated from SRIM simulations (Ref. 73).

${ }^{\mathrm{b}}$ From Ref. 60

${ }^{\mathrm{c}}$ From Ref. 78

Table III shows that for silicon secondary electron yields by ion irradiation are about a factor of 5 to 50 higher than by electron irradiation.

\section{Radial density distribution of surface interactions}

For both electron beam and ion beam induced processes, the minimum dimensions of the structures that can be produced are larger than the incident beam diameters. The radial flux distributions of the emitted secondary and backscattered electrons and of the excited surface atoms determine the minimum dimensions of the structures that can be fabricated. Calculations of these radial distributions rely on Monte Carlo simulations. Figure 8(a) shows the radial flux distributions for electron bombardment of bulk silicon with a zero diameter beam. The emitted secondary electron distribution comprises all electrons with an exit energy $\leqslant 50 \mathrm{eV}$ and the backscattered distribution all electrons with energies $\geqslant 50 \mathrm{eV}$ until the incident electron energy. The $\mathrm{MC}$ program MOCASIM (Ref. 81) was used and works with small-angle and large-angle Mott cross sections for inelastic energy losses and with Gryzinski and Moller cross sections (inner shell excitation $^{82-85}$ for inelastic collisions. It applies a straight line approximation with exponential decrease for secondary electron generation and parametrized escape depths and exit energies. ${ }^{86,87}$ The radial density of secondary electrons is one to two orders of magnitude larger than backscattered elec- trons near the beam center. The radial distribution of backscattered electrons extends to the range $R_{\mathrm{BSE}}$ as defined in Appendix A. A broad contribution of secondary electrons being generated from backscattered electrons is also visible. Note that the long tails of the distributions are not generated when using membrane substrates. According to Fig. 8(b), the emitted secondary electron flux contains a very peaked contribution at $r=0$ with a FWHM in the order of $0.1 \mathrm{~nm}$ due to secondary electrons generated by incident primary electrons. The strong confinement is due to the small average exit depth limiting the exit cone of secondary electrons to small angles around the penetrating primary electron beam. At the full width containing 50\% of secondary electrons, the flux decays by almost two orders of magnitude. The central region of the distribution can be fitted, for instance, with $f_{\mathrm{SE}}(r)=A \exp (-B r-C /(r+D)) .{ }^{56}$ Convolution of curves in Fig. 8 with an incident beam distribution gives the real emitted radial distribution.

Analogous MC simulations for excited surface atoms generated by incident primary ions could be performed but we would like to mention an experimental approach to estimate the extent of this distribution proposed by Ref. 67: from measured chemical FIB deposition yields $Y_{D}$ [deposited metal atoms/ion], the diameter of the excited surface atom distribution is obtained by calculating how much surface the number of decomposed molecules initially covered, namely,
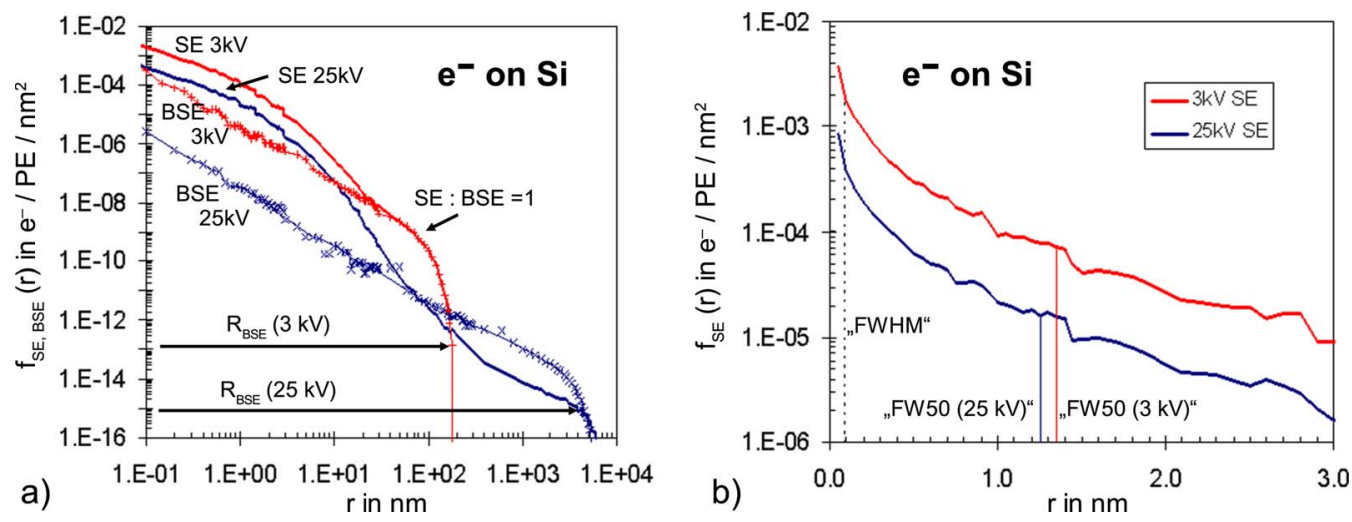

FIG. 8. MC-simulated radial distributions of emitted secondary electrons (SEs) and backscattered electrons (BSEs) per primary electron (PE): Zero diameter electron beam impinging normal with 25 and $3 \mathrm{keV}$ at $r=0$ on planar bulk silicon. The SE exit depth was taken as $5.2 \mathrm{~nm}$ and the mean exit energy as $70 \mathrm{eV}$. (a) Double-log plot showing the SE distribution tails extending to the range of backscattered electrons $R_{\mathrm{BSE}}$. (b) Log plot of the secondary electron flux. Comparison of the FWHM and FW50 (full width containing 50\% secondary electrons). 
TABLE IV. Estimates of nonlocal effect ranges due to emitted secondary electrons $\left(d_{\mathrm{SE}}\right)$ and excited surface atoms $\left(d_{\mathrm{ESA}}\right)$ according to Eq. (2.3) using measured deposition yields $Y_{D}$ or chemical etch yields $Y_{\text {etch. }}$. The molecule diameter $\delta_{m}$ is taken from table VI. The deposition yield $Y_{D}$ is not uniquely define in literature. Here we adapt the definition of Dubner (Ref. 57), which corrects for the simultaneous sputtering of the deposit.

\begin{tabular}{lcccccc}
\hline \hline Particles & $\begin{array}{c}E \\
(\mathrm{keV})\end{array}$ & Molecule & $m$ & $\begin{array}{c}\delta_{m} \\
(\mathrm{~nm})\end{array}$ & $Y_{D}, Y_{\text {etch }}$ & $\begin{array}{c}d_{\mathrm{ESA}}, d_{\mathrm{SE}} \\
(\mathrm{nm})\end{array}$ \\
\hline $\mathrm{Kr}+$ & 50 & $\left(\mathrm{CH}_{3}\right)_{2} \mathrm{Au}(\mathrm{tfa})$ & 1 & 0.35 & $24^{\mathrm{a}}$ & 1.7 \\
$\mathrm{Ga}+$ & 42 & $\mathrm{~W}(\mathrm{CO})_{6}$ & 1 & 0.33 & $5.3^{\mathrm{b}}$ & 0.8 \\
$\mathrm{Ga}+$ & 25 & $\mathrm{I}_{2}(\mathrm{Si} \mathrm{etch})$ & 2 & 0.5 & $40^{\mathrm{c}}$ & 6.3 \\
$e^{-}$ & 5 & MeCpPt(Me) & 1 & 0.4 & $0.001^{\mathrm{d}}$ & $<0.1$ \\
$e^{-}$ & 25 & $($ hfac)CuVTMS & 1 & 0.8 & $0.02^{\mathrm{e}}$ & 0.1 \\
\hline \hline
\end{tabular}

${ }^{\mathrm{a}}$ Reference 67.

${ }^{\mathrm{b}}$ Reference 68

${ }^{\mathrm{c}}$ Reference 89

${ }^{\mathrm{d}}$ Reference 90

${ }^{\mathrm{e}}$ Reference 91.

$$
d_{\mathrm{ESA}} \cong m^{-1} Y_{D}^{1 / 2} \delta_{m},
$$

where $\delta_{m}$ is the molecule diameter and $m$ is the metal stoichiometry in the molecule. This approach assumes one complete adsorbed monolayer. If this is not the case, the range would become larger. This approach can be extended to both gas-assisted FIB etching (where $\mathrm{m}^{-1}$ would now denote the number of molecules involved in the etch reaction) and FEB deposition and etching (see Table IV).

From Table IV and Eq. (2.3) follows that the size of the excited surface atom distribution is in the sub-10-nm range; however, to our knowledge this resolution has not yet been experimentally verified by gas-assisted FIB experiments. Of note is that sub-5-nm resolution was obtained for FIB milling (without gas) on membranes. ${ }^{55}$ The size of the emitted secondary electron flux is in the sub-1-nm range, which is in accordance with the FWHM value obtained from MC simulations [see Fig. 8(b)]. The subnanometer-resolution for gasassisted FEB was already demonstrated with deposition from $\mathrm{W}(\mathrm{CO})_{6}{ }^{92}$

\section{B. Electron interaction with molecules}

There are numerous interaction mechanisms during electron impact on molecules, such as dissociation, stimulated desorption, polymerization, and sputtering. For most mechanisms further submechanisms can be identified. Each is described by an energy dependent cross sections $\sigma(E)$. However, irradiation data of relevant molecules used for FEB and FIB deposition and etching are very rare.

On the other hand, the knowledge of the cross section is crucial in gaining full control over the purity, the throughput, and the precision of the FEB and FIB gas-assisted nanoscale fabrication process. It depends on the cross section's energy dependence which part of the energy spectrum and its respective radial distribution will determine the dissociation rate, ultimate resolution, and purity of the deposit or etch process.

Due to the lack of data mainly two practical approaches are pursued:
(1) The first approach is to determine one constant deposition or etch cross section from FEB or FIB experiments at a given incident energy. Thereby the wealth of irradiation induced processes due to the emitted energy spectrum is condensed into one measurable physical parameter. Evidently, the unique identification of the governing interaction channel for deposition or etching is not answered from this approach. Nevertheless, a reproducible material system parameter under specific irradiation conditions is obtained. This approach is discussed in more detail in Secs. III B and III D.

(2) The second approach adapts the very few obtainable energy dependencies from gas phase measurements and melds them into a generic type of energy dependence for use in MC simulations. However, it remains an open question if the chosen interaction channel with its energy dependence is characteristic for the deposition or etch experiment.

In this section we briefly review energy dependencies of cross sections from gas phase measurements and adsorbed molecules. Gas phase interactions with charged particles can occur above the substrate (see Sec. II D 4) and have been studied in detail. Interaction channels with adsorbed molecules are similar but often reduced due to additional relaxation paths with the surface.

\section{Electron interaction with gas phase molecules}

The electronically excited dissociation of a few halogenated etch gas molecules relevant in semiconductor plasma processing by electron impact was reviewed by Christophorou and Olthoff. ${ }^{93}$ Figure 9 shows three dissociation mechanisms for a $\mathrm{CF}_{4}$ gas phase molecule: (a) The dissociative electron attachment is a resonant interaction in an energy window close to the emitted secondary electron intensity peak. It has its peak value when the electron energy matches the energy of the lowest unoccupied molecular orbital. It was shown for more complex alcohol molecules that dissociative 


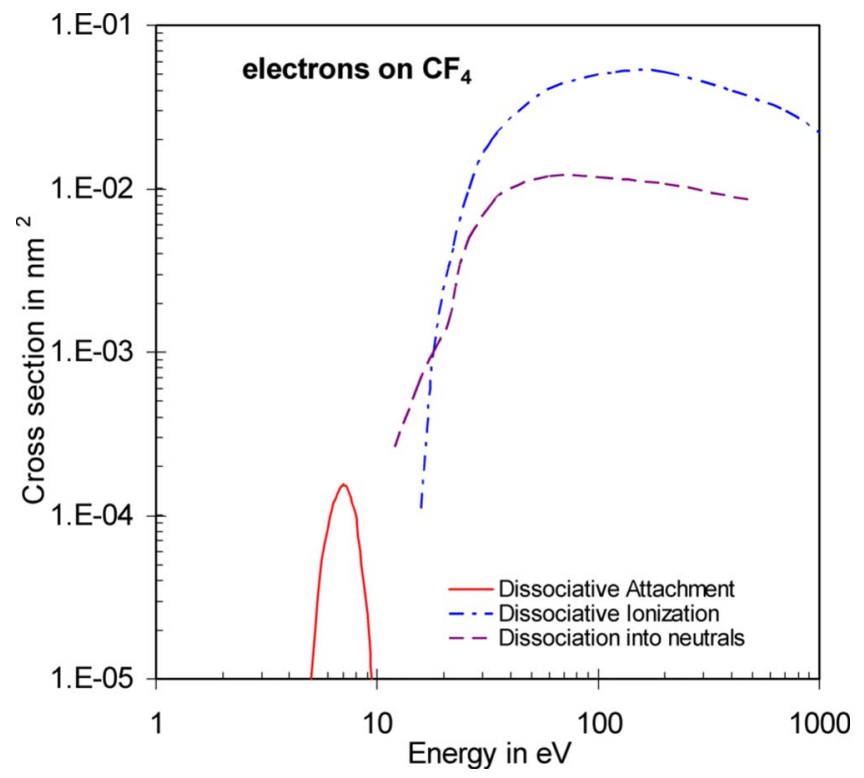

FIG. 9. Electron impact total cross sections for gas phase $\mathrm{CF}_{4}$ (reproduced from Ref. 92).

electron attachment is state selective, i.e., depending on the incident electron energy the hydroxyl group is dissociated $(6.5 \mathrm{eV})$ or the alkyl groups $(8-9 \mathrm{eV}) .^{94}$

(b) The cross section for direct dissociation into ions has a shape with threshold energy at $>10 \mathrm{eV}$ corresponding to the ionization energy. The peak value is situated around $70-100 \mathrm{eV}$ and decays with increasing energy. (c) The cross section for dissociation into neutrals has the same features. The threshold energy is related to the molecule bond enthalpy (or dissociation energy) being situated at around $10 \mathrm{eV}$. Each of the electron impact dissociation mechanisms can lead to several dissociation products (or can have several dissociation channels), which were also studied in detail for the molecule $\mathrm{CF}_{4}{ }^{95}$ The sum over all channels for one mechanism gives the total cross section of this mechanism.

The energy dependence of the non resonant cross sections shown in Fig. 9 has been described by different models, such as the binary-encounter-Bethe model for ionization ${ }^{96}$ or by generic equations for dissociation of hydrocarbons. ${ }^{97}$ Both are mainly used for MC simulations. ${ }^{20,66}$

The variety of fragments due to dissociative ionization as function of energy of a relevant metal containing molecule, $\mathrm{Co}_{2}(\mathrm{CO})_{8}$, is shown in Fig. 10 and was obtained from mass spectrometry measurements. ${ }^{98}$ It becomes evident that it is difficult to generate a simple generic cross section that adequately simulates the deposition process.

Other spectra for other carbonyls can be found in Refs. 98-102 and for $\mathrm{Ni}\left(\mathrm{PF}_{3}\right)_{4}$ in Ref. 103. The onset of each ionic fragment curve corresponds to its appearance potential (ionization energy threshold).

Mass spectra taken at constant energy (mostly $70-100 \mathrm{eV}$ ) of most molecules can be accessed in databases. They figure relative abundances of ionic fragment species. As an estimate which deposit composition can be expected from dissociative ionization, we propose to sum up the com-

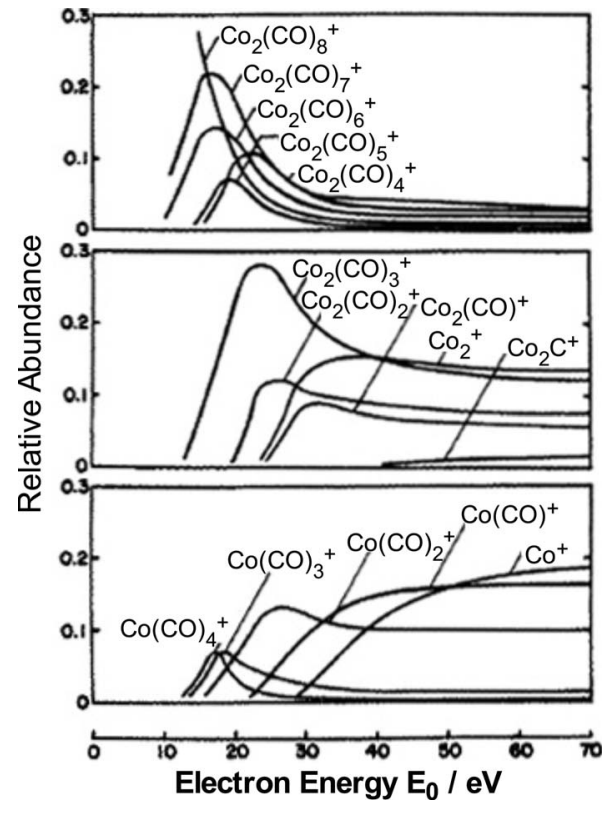

FIG. 10. Relative abundance of positively charged ionization fragments from a gaseous $\mathrm{Co}_{2}(\mathrm{CO})_{8}$ parent molecule vs electron impact energy $E_{0}$. The decomposition of $\mathrm{Co}_{2}(\mathrm{CO})_{8}$ proceeds in a series of successive $\mathrm{CO}$ group removals with specific threshold and peak energies. For better visibility the fragments are grouped into three graphs starting with the successive removal of $\mathrm{CO}$ groups from $\mathrm{Co}_{2}(\mathrm{CO})_{8}$ (top and middle graph). The bottom graph shows successive $\mathrm{CO}$ group removal from the monomer ion $\mathrm{Co}(\mathrm{CO})_{4}^{+}(\mathrm{re}-$ produced from Ref. 98).

positions of all nonvolatile species from the mass spectrum weighted by their respective relative abundances. For example, this approach would suggest for $\mathrm{Co}_{2}(\mathrm{CO})_{8}$ that the FEB deposit contains 22 at. \% Co and 39 at. \% C and $\mathrm{O}$ or has a stoichiometry of about $\mathrm{Co}_{2}(\mathrm{CO})_{4}$. The metal content is close to experimental findings at low beam currents and lowaspect-ratio deposits where beam heating effects can be excluded. ${ }^{104}$ Also for FEB deposition using $\left[\mathrm{RhCl}\left(\mathrm{PF}_{3}\right)_{2}\right]_{2}$ as a precursor, an accordance of the mass spectrum and the composition is found. ${ }^{105}$ However, this estimate will not work for molecules, the dissociation channel of which is not dominated by dissociative ionization.

\section{Electron interaction with adsorbed molecules}

A major difference that distinguishes electron irradiation processes at surfaces from gas phase dissociative processes is that the underlying material provides additional channels for electronic relaxation that are not available in the gas phase. ${ }^{106}$ Dissociation cross sections of molecules for irradiation might be much smaller for adsorbed molecules due to fast surface relaxation processes taking place. ${ }^{107} \mathrm{~A}$ second major difference is that in the condensed phase, electron scattering phenomena are dominated by the effects of multiple scattering; an electron may scatter many times before it passes through the film, is reflected back into vacuum, or becomes trapped. Consequently, a determination of an absolute cross section per scatter event is not easily possible. Effective cross sections that quantitatively describe the scattering of the ensemble of molecules within condensed 

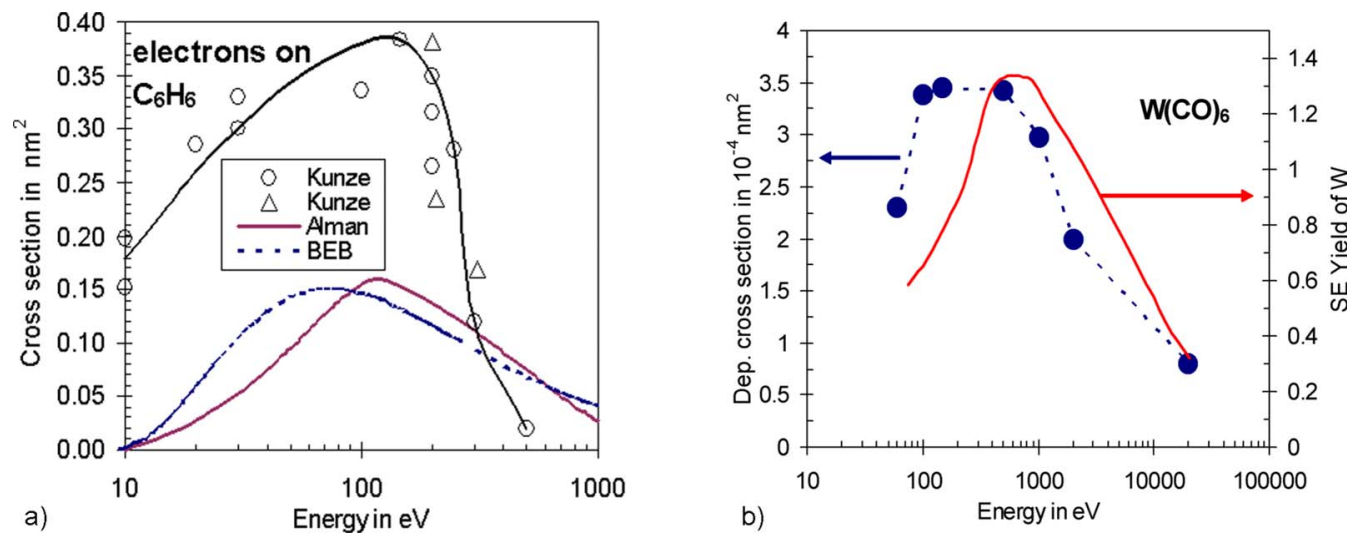

Fig. 11. (a) Electron impact deposition cross section of benzene [reproduced from Kunze (Ref. 110)]. For comparison the generic ionization cross sections according to Alman (Ref. 97) and the BEB theory (Ref. 96) are shown. (b) Electron impact deposition cross section for W(CO) 6 and secondary electron (SE) yield of pure W [reproduced from Hoyle (Ref. 64)].

halogeno-methane films and water films were reviewed. ${ }^{108} \mathrm{~A}$ third major difference to gas phase dissociation is that additional relaxation channels are also provided inside the condensed film and that new channels become operative, for instance, when dissociation products react with neiboring molecules. ${ }^{109}$

Apart from the scarce data on gas phase electron impact cross sections for relevant molecules, equivalent data for adsorbed molecules are also very few. Figure 11(a) compares cross section energy dependencies obtained from electron beam induced deposition experiments with benzene ${ }^{110}$ with the generic cross section of $\mathrm{Alman}^{97}$ and the binaryencounter-Bethe (BEB) cross section. ${ }^{96}$ The differences with the experiment are obvious and can be probably attributed to the fact that the dominant channel for deposition is a polymerization reaction and not (only) dissociation into ions. At high energies the decay is much stronger than anticipated by the models for dissociative ionization.

Figure 11(b) shows a comparison of the $\mathrm{W}(\mathrm{CO})_{6}$ FEB deposition cross section with the secondary electron yield. The discrepancy at low electron energies was attributed to possible gas reactions that might have contributed to the deposition. ${ }^{64}$ The $\mathrm{W}(\mathrm{CO})_{6}$ deposition cross section is a factor 1000 smaller than the deposition cross section of $\mathrm{C}_{6} \mathrm{H}_{6}$. This might be related to different efficiencies of deposition channels: $\mathrm{W}(\mathrm{CO})_{6}$ must be dissociated to tungsten or tungsten-rich nonvolatile fragments, whereas $\mathrm{C}_{6} \mathrm{H}_{6}$ molecules become nonvolatile by polymerization triggered by electron induced radical formation.

There are a few data sets for dissociative electron attachment of adsorbed molecules measured in ultrahigh vacuum conditions and well defined surfaces. For the molecule (hfac)Cu-VTMS adsorbed on (111)Si, a threshold energy of $4 \pm 0.5 \mathrm{eV}$ and the inset of a possible direct ionization mechanism at around $23 \mathrm{eV}$ were observed. ${ }^{111}$ Due to the existence of many possible resonant energies in the $\mathrm{Cu}$ precursor molecule, there is no sharp resonance maximum. An observed shift to lower binding energy for fluorine is attributed to rebonding to different atoms in the electron impact deposit. Oxidation of hydrogen-passivated $\mathrm{Si}(111)$ sur- faces induced by dissociative electron attachment of physisorbed $\mathrm{H}_{2} \mathrm{O}$ showed a threshold at around $5 \mathrm{eV}$ and a peak of about $5 \times 10^{-17} \mathrm{~cm}^{2}$ at $11 \mathrm{eV}$. The use of hydrogenated silicon as a prototype for resistless e-beam lithography with linewidths below $60 \mathrm{~nm}$ was demonstrated. ${ }^{112}$ The resolution was found to depend on the electron beam diameter and the range of secondary electrons generated by the incident electrons.

The dissociation of $\mathrm{Ni}(\mathrm{CO})_{4}$ on $\mathrm{Ag}(111)$ by $100-300 \mathrm{eV}$ electrons showed desorption of positive ions and excited neutrals together with the conversion into unidentified $\mathrm{Ni}_{x}(\mathrm{CO})_{y}$ species on the surface. ${ }^{113}$ Carbonyl groups thermally desorb from these surface bound species at 200-400 K, leaving a Ni deposit on the surface. The reported total cross section for $\mathrm{Ni}(\mathrm{CO})_{4}$ dissociation is about $2 \times 10^{-16} \mathrm{~cm}^{2}$ in this energy range. It remains an open question of how applicable these data are on "ill-defined" surfaces, such as surfaces of deposits or amorphized top layers, being formed while FEB and FIB gas-assisted processing.

\section{Electron stimulated desorption}

Electron stimulated desorption (ESD) is initiated by an electronic excitation. Bonds of surface species are excited and can result in desorption of ions, neutrals, or vibrationally excited (metastable) species from the surface. Madey ${ }^{114}$ resumed the related cross sections as follows: the maximum cross sections from desorption of ions from surfaces $\left(10^{-23}-10^{-20} \mathrm{~cm}^{2}\right)$ are generally smaller than those for desorption of neutral species $\left(10^{-20}-10^{-18} \mathrm{~cm}^{2}\right)$; both are smaller than typical cross sections for gas phase dissociative ionization $\left(10^{-16} \mathrm{~cm}^{2}\right.$ for $100 \mathrm{eV}$ electrons). Threshold energies for desorption can be as low as $5 \mathrm{eV}$ (neutral molecules); for ion desorption via valence and shallow-core electrons, excitations have thresholds of $15 \mathrm{eV}$ or more. Electron induced desorption cross sections depend on the surface and the adsorbed molecule. We give as an example the following desorption cross sections at an electron energy of $100 \mathrm{eV}$ and 
a surface temperature of $350 \mathrm{~K}$ : $\sigma\left(\mathrm{O}_{2}\right.$ on $\left.\mathrm{Si}\right)=6 \times 10^{-17}$ $\mathrm{cm}^{2} \sigma(\mathrm{CO}$ on $\mathrm{Pd})=1 \times 10^{-17} \mathrm{~cm}^{2}$, and $\sigma\left(\mathrm{Cl}_{2}\right.$ on $\left.\mathrm{Pd}\right)=1.6$ $\times 10^{-17} \mathrm{~cm}^{2}$. 115

The related models describing electron stimulated desorption are the Knotek-Feibelman model ${ }^{116,117}$ for ionic substrates and the Menzel-Gomer-Redhead model for covalent adsorbates. ${ }^{118,119}$ According to the Knotek-Feibelman model, the materials most likely to decompose are maximal valency compounds in which the cation and anion have large Pauling electronegativity differences: $\mathrm{TiO}_{2}, \mathrm{~V}_{2} \mathrm{O}_{5}, \mathrm{SiO}_{2}, \mathrm{Al}_{2} \mathrm{O}_{3}$, $\mathrm{WO}_{3}$, and $\mathrm{MoO}_{3}$. Halides lose halogen during electron bombardment and have been shown to work as high resolution inorganic resists. ${ }^{120,121}$ Electron stimulated desorption mechanisms of hydrogen and fluorine from organic molecules was investigated in Ref. 122. The role of Auger electron cascades leading to electron vacancies and successive displacements in covalent crystals or stimulated desorption in ionic species was reviewed in Ref. 123. Electron nanoetching was demonstrated, for example, on membranes of $\mathrm{Al}_{2} \mathrm{O}_{3}, \mathrm{MgO}, \mathrm{Si}$, and $\mathrm{Al}$, resulting in holes with nanometer resolution in diameter using $40-100 \mathrm{keV}$ electrons. ${ }^{124}$ The same paper demonstrates e-beam milling of $4 \mathrm{~nm}$ diameter holes in $\mathrm{AlF}_{3}$.

Excellent review articles ${ }^{107,125,126}$ on desorption induced by electronic transition and ESD give further information for the interested reader. However, compared to "surface science" conditions (crystallographically defined surfaces and ultrahigh vacuum conditions), the situation is kind of ill defined for most gas-assisted FEB and FIB processes since the deposit surface is neither crystallographic nor "clean." In fact, the dissociation process very probably leads to complex surface situations with ligands partially fragmented or intact ligands adsorbed on partially decomposed surfaces. Thus we think that at best trends can be identified from above surface science experiments.

\section{Physical sputtering due to electrons}

Here we discuss a purely mechanical process of material removal by electron bombardment. In contrast to the above discussed mechanisms, no electronic excitation is involved. The maximum kinetic energy that can be transferred to an atom with mass $m_{a}$ in a collision with an electron having an energy $E$ and mass $m_{e}$ is $(E<200 \mathrm{keV})$

$$
E_{\max }=4 E \frac{m_{e} m_{a}}{\left(m_{e}+m_{a}\right)^{2}} \cong 4 E m_{e} / m_{a} .
$$

The momentum transfer involved in this high-angle collision is mainly in the incident direction. ${ }^{127}$ In the case of electron "transparent" structures (e.g., membranes, nanotubes, and nanowires), it is believed that the sputtering crater forms predominantly at the beam-exit surface. Sputtering occurs when the energy transfer $E_{\max } \geqslant E_{s}$, where $E_{s}$ is the surface binding energy often inferred from the sublimation energy per atom. Thus the incident electron energy $E_{0}$ must be larger than $E_{0} \geqslant\left(E_{s} / 4\right)\left(m_{a} / m_{e}\right)$ to cause physical sputtering. For example, a $\mathrm{Si}$ atom has a surface binding energy of $E_{s}$ $=4.7 \mathrm{eV}$ and can be removed with an incident electron en- ergy of $E_{0} \geqslant 57 \mathrm{keV}$. The sputter cross section derived from a nonrelativistic Rutherford-scattering model is ${ }^{128}$

$$
\sigma=3.54 \times 10^{-3} \frac{Z^{2}}{A E_{0}}\left(1 / E_{S}-1 / E_{\max }\right),
$$

with $E_{0}$ and $E_{s}$ in $\mathrm{eV}, \sigma$ in $\mathrm{nm}^{2}$, and $A$ is the atomic weight of the target atom. Incident energies larger than a few tens of kilovolts are needed to overcome the threshold energies in the range of about $5 \mathrm{eV}$. The cross section for sputtering of a carbon membrane by $80 \mathrm{keV}$ electrons is $1.8 \times 10^{-22} \mathrm{~cm}^{2} .{ }^{128}$ At incident electron energies $E_{0}>100 \mathrm{kV}$, the energy transfer reaches the magnitude of displacement energies $E_{D}$ for atoms in crystals, which can be used to crystallize amorphous silicon, ${ }^{129}$ to reinforce carbon nanotubes bundles, ${ }^{130,131}$ or to sculpt FEB deposited Si-O-C nanowires. ${ }^{132}$

\section{Ion interaction with molecules}

Energetic ions incident on a surface on which gas molecules are adsorbed can produce a number of effects: for example, desorption of the molecules, dissociation of the molecule, or reaction of the molecules with the substrate material. The latter two effects are exploited in FIB applications. If ions are incident on the surface that has some $\mathrm{W}(\mathrm{CO})_{6}$ adsorbed, the molecules dissociate, forming conducting deposits of a mixture of tungsten and carbon. On the other hand if xenon difluoride is absorbed on, say, silicon, then the fluorine will react with the substrate and cause it to be removed as $\mathrm{SiF}_{4}$ or other gaseous species. Here we will briefly review a mechanistic approach describing the interactions with molecules.

\section{Ion interaction with adsorbed molecules}

For ion-impact dissociation an energy dependence of the cross section was proposed by Dubner ${ }^{57}$ based on a simple kinetic energy transfer model between an excited surface atom, of mass $m_{\mathrm{ESA}}$, and the atoms of the adsorbed molecule. The maximum kinetic energy that can be transferred to an atom with mass $m_{a}$ in a (nonrelativistic) collision is

$$
E_{\mathrm{max}}=4 E_{\mathrm{ESA}} \frac{m_{\mathrm{ESA}} m_{a}}{\left(m_{\mathrm{ESA}}+m_{a}\right)^{2}},
$$

where $E_{\mathrm{ESA}}$ is the energy of an excited surface atom due to a collective collision cascade terminating at the surface (see Sec. II B). The energy dependence of the dissociation cross section for a given atom of the molecule according to Ref. 57 in our notation is

$$
\sigma(E)=\sigma_{0}\left(1-E_{\mathrm{diss}} / E_{\max }\right),
$$

where $E_{\text {diss }}$ is taken as the dissociation or bond energy of the precursor. The total cross section for the molecule is obtained by summing the individual cross sections of the constituting atoms according to their stoichiometry and their relative area covered by each atom in the molecule (see Fig. 12). For excited surface atom energies $E<E_{\max }$, the molecule is not dissociated and $\sigma(E)=0$. The value $\sigma_{0}$ is asymptotically reached with increasing energy of the excited surface atom 


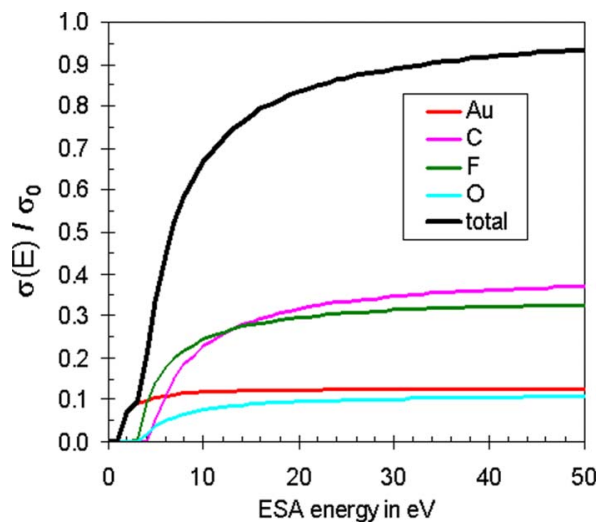

FIG. 12. Plot of $\sigma(E) / \sigma_{0}$ vs energy of excited surface atom according to the kinetic energy transfer model. The excited surface atom is gold and the precursor molecule $\mathrm{Me}_{2} \mathrm{Au}(\mathrm{hfa})$. The dissociation energy of this molecule was assumed to be $1 \mathrm{eV}$ (adapted from Ref. 57).

and represents a constant for a given incident energy. We note here the analogy with the cross section for electron sputtering in Eq. (2.5).

The energy dependence of the cross section from Fig. 12 and the energy distribution of excited surface atoms from Fig. 12(b) allow the energy integral in Eq. (2.1a) and (2.1b) to be evaluated for ions. The weak point in this model is that it does not answer the question how the energy transfer from the excited surface atom to the molecule proceeds. The postulated atom-atom collision is surely oversimplified but good agreement with experimental yield measurements was found. ${ }^{57}$ An alternative model describing the ion impact as a thermal spike was also discussed but does not fit the data as well as the binary collision model.

\section{Physical sputtering due to ions}

Of all of the processes we are discussing, focused ion beam milling is probably the one most widely used. The gallium ion beam is used to remove material of any type and does not require chemical reactions. In the semiconductor industry FIB milling is used in failure analysis, circuit restructuring, and TEM sample preparation. A number of other applications range from scanning probe tip trimming to nanohole fabrication in gold films used to observe effects of light interaction with surface plasmons, see Sec. VII.

Sputtering occurs when the energy transfer $E_{\max } \geqslant w E_{s}$, where $E_{s}$ is the surface binding energy often inferred from the sublimation energy per atom. Heats of sublimation of all elements are summarized in Ref. 133. From experiments the prefactor was found to vary between $w=1$ and 6.7 according to the mass ratio of ion and target atom. ${ }^{134}$ Accordingly, the threshold incident energy is $E_{0}=w E_{s} / E_{\max }=0.25 w E_{s}\left(m_{\text {ion }}\right.$ $\left.+m_{a}\right)^{2} /\left(m_{\mathrm{ion}} m_{a}\right)$. For $\mathrm{Si}$ the surface binding energy $E_{s}$ $=4.7 \mathrm{eV}$ and, consequently, an incident $\mathrm{Ga}$ ion with an incident energy of $36.2 \mathrm{eV}$ can already cause sputtering. Since FIB processing is predominantly performed at $\mathrm{keV}$ energies, the maximum energy transfer also exceeds largely the bulk displacement energy ( $\mathrm{Si}: 14 \mathrm{eV}$ ), which causes collision cascades of dislocated target atoms along the primary ion tra-
TABLE V. Sputter yields for normal incidence, different ions, incident energies, and materials.

\begin{tabular}{|c|c|c|c|c|}
\hline Substrate & Ion & $\begin{array}{c}\text { Energy } \\
(\mathrm{keV})\end{array}$ & $\begin{array}{c}\text { Yield } \\
\text { (atoms/ion) }\end{array}$ & Ref. \\
\hline $\mathrm{Si}$ & $\mathrm{Ga}^{+}$ & 30 & $3.1 \pm 0.8$ & 151 \\
\hline $\mathrm{Si}$ & $\mathrm{Ga}^{+}$ & 30 & $1.92 \pm 0.16$ & 139 \\
\hline $\mathrm{Si}$ & $\mathrm{Ga}^{+}$ & 25 & 2.6 & 152 \\
\hline $\mathrm{Si}$ & $\mathrm{Ga}^{+}$ & 25 & $3.9 \pm 0.4$ & 136 \\
\hline $\mathrm{Si}$ & $\mathrm{Kr}^{+}$ & 25 & 3.1 & 150 \\
\hline $\begin{array}{l}\text { Diamond } \\
(100)\end{array}$ & $\mathrm{Ga}^{+}$ & 20 & 2 & 153 \\
\hline Diamond & $\mathrm{Ga}^{+}$ & 50 & 2.3 & 154 \\
\hline $\begin{array}{l}\text { Hard amorphous } \\
\text { carbon }\end{array}$ & $\mathrm{Ga}^{+}$ & 50 & 2.6 & 154 \\
\hline \multirow[t]{4}{*}{$\mathrm{GaN}$} & \multirow[t]{4}{*}{$\mathrm{Ga}^{+}$} & 15 & 5.2 & \multirow[t]{4}{*}{155} \\
\hline & & 30 & 6.2 & \\
\hline & & 50 & 6.8 & \\
\hline & & 70 & 6.7 & \\
\hline $\mathrm{InP}$ & $\mathrm{Ga}^{+}$ & 30 & 7.5 & 155 \\
\hline GaAs & $\mathrm{Ga}^{+}$ & 30 & 4.8 & 155 \\
\hline $\mathrm{SiC}$ & $\mathrm{Ga}^{+}$ & 30 & 2.5 & 155 \\
\hline $\mathrm{Al}_{2} \mathrm{O}_{3}$ & $\mathrm{Ga}^{+}$ & 30 & 1.9 & 155 \\
\hline $\mathrm{Au}$ & $\mathrm{Ga}^{+}$ & 100 & 32 & 139 \\
\hline $\mathrm{Au}$ & $\mathrm{Ga}^{+}$ & 40 & $15.7 \pm 1.3$ & 58 \\
\hline $\mathrm{Au}$ (plated) & $\mathrm{Ga}^{+}$ & 25 & $18 \pm 3$ & 136 \\
\hline Au (evap.) & $\mathrm{Ga}^{+}$ & 25 & $23 \pm 5$ & 136 \\
\hline $\mathrm{Au}$ & $\mathrm{Kr}^{+}$ & 25 & 20 & 150 \\
\hline W (RF-sputt.) & $\mathrm{Ga}^{+}$ & 25 & $5 \pm 0.7$ & 136 \\
\hline $\mathrm{W}$ & $\mathrm{Kr}^{+}$ & 22 & 4.1 & 150 \\
\hline $\mathrm{Al}$ & $\mathrm{Ga}^{+}$ & 30 & $1.1-4.5$ & 139 \\
\hline \multirow[t]{2}{*}{$\mathrm{Al}$} & \multirow[t]{2}{*}{$\mathrm{Si}^{+}$} & 30 & -0.26 & \multirow[t]{2}{*}{156} \\
\hline & & 120 & -0.09 & \\
\hline $\mathrm{SiO}_{2}$ & $\mathrm{Ga}^{+}$ & 68 & 2.0 molec./ion & 157 \\
\hline $\mathrm{SiO}_{2}$ & $\mathrm{Ga}^{+}$ & 25 & 0.84 & 136 \\
\hline $\mathrm{SiO}_{2}$ & $\mathrm{Ga}^{+}$ & 30 & 0.85 & 158 \\
\hline $\mathrm{SiO}_{2}$ & $\mathrm{Ga}^{+}$ & 30 & 2.0 & 155 \\
\hline
\end{tabular}

jectory (amorphization). When they reach the surface with a normal energy component larger than $4.7 \mathrm{eV}$, they can leave the surface as sputtered atom [see also Fig. 7(b)].

The fundamental quantity that describes the milling rate at a given ion current is sputter yield, i.e., the average number of substrate atoms (or molecules in the case of compound substrates) removed from the surface by each incident ion. The sputter yield of a number of materials is shown in Table $\mathrm{V}$; the yield for $\mathrm{Kr}^{+}$ions is also included for comparison since $\mathrm{Kr}^{+}$is the noble gas ion closest to $\mathrm{Ga}^{+}$in mass. Note that the yield of $\mathrm{Al}$ milled with $\mathrm{Si}$ is negative, i.e., the material swells due to irradiation. The relation of the sputter yield $Y_{S}$ in units of removed atoms per ion and the sputter rate $R_{S}$ in units of removed volume per charge (often given in $\left.\mu \mathrm{m}^{3} / \mathrm{nC}\right)$ is $R_{S}=Y_{S} M /\left(\rho N_{A} e_{0}\right)$, where $N_{A}$ is Avogadro's constant, $e_{0}$ is the elementary charge, and $M$ and $\rho$ are the molar mass and density of the sputtered material. While the sputter yield or rate would seem to be a straightforward quantity that is easily measured, this is not always the case, particularly with focused ion beams. Possible complications are as follows. 


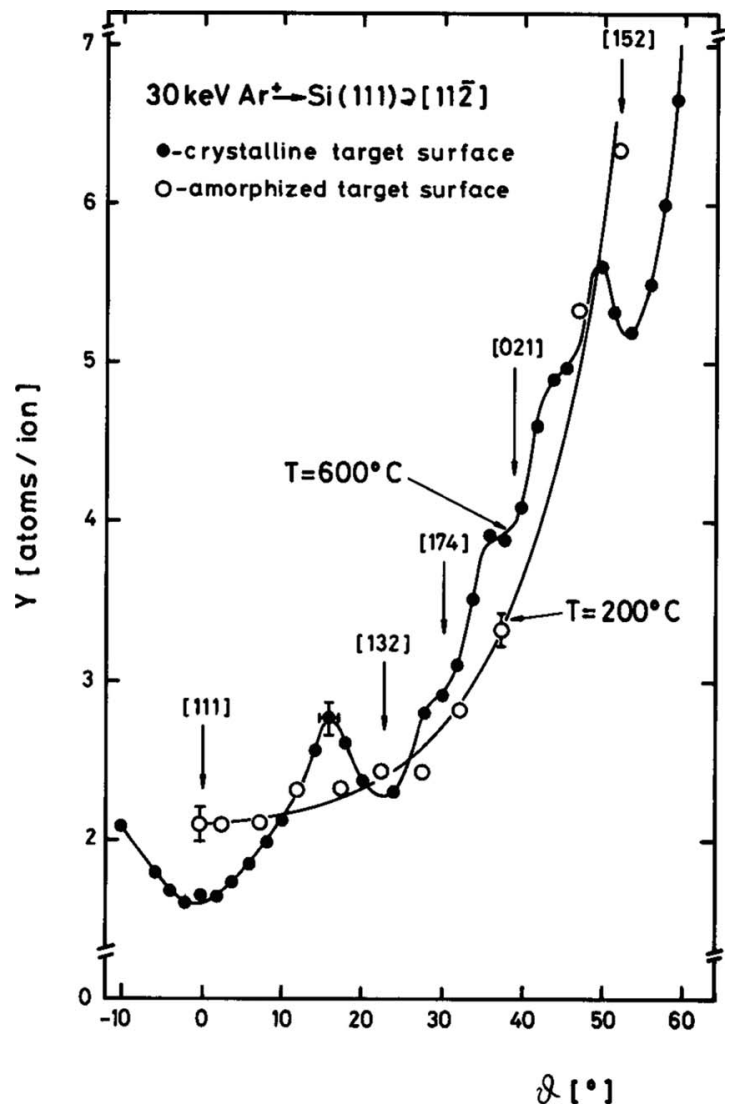

FIG. 13. Milling yield as a function of angle of incidence of $30 \mathrm{keV}$ Ar ions on a (111) surface of $\mathrm{Si}$, rotated about the (112) axis. The milling yield is seen to drop when the ions are incident along a crystal symmetry direction. For an amorphized target the curve is smooth (from Ref. 135).

As a result of ion channeling, the sputter yield depends on the orientation of the crystal axis with respect to the incident ion beam (see Fig. 13). Thus, in a polycrystalline sample, each grain may mill at a different rate and a milled surface may become very rough as illustrated in Fig. 14. This adds a complication to the measurement of yield for metal films, which are usually polycrystalline.

(2) Surface reaction with residual gas atoms may play a role, particularly if a large area is scanned with a low current beam. For example, at $10^{-6}$ mbar, which is a typical pressure in FIB chambers, $1 \mathrm{ML}$ can form in $1 \mathrm{~s}$. In other words the impingement rate of molecules

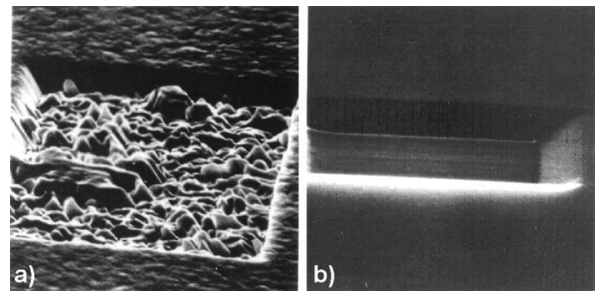

FIG. 14. (a) Box of $10 \times 10 \mu \mathrm{m}^{2}$ milled in polycrystalline gold using a repetitive serpentine scan such that each pass removed only a fraction of a monolayer. Ion beam at $30^{\circ}$ from normal at $25 \mathrm{keV}$ and $283 \mathrm{pA}$. (b) A similar box milled in single crystal silicon (from Ref. 136).

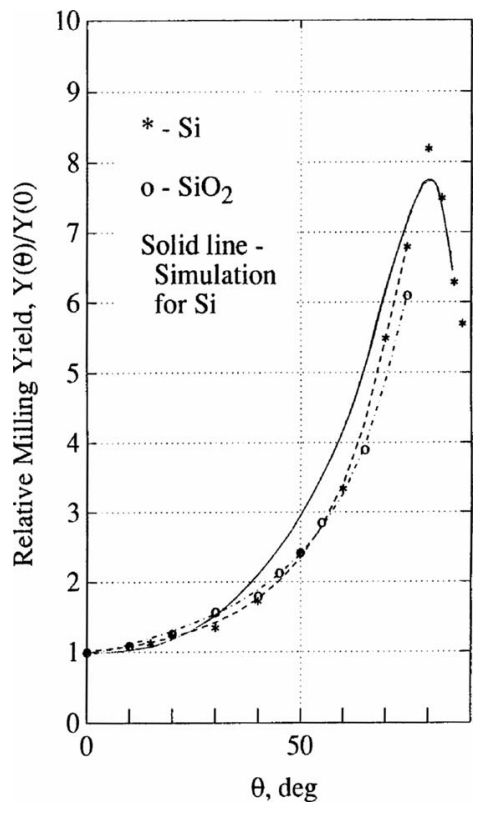

FIG. 15. Relative milling yield as a function of angle from normal for silicon dioxide and for silicon. Note that both experiment and simulation show a decrease in yield as the angle approaches $90^{\circ}$ (from Ref. 136).

is about $5 \times 10^{14} \mathrm{~cm}^{-2} \mathrm{~s}^{-1}$, according to Eq. (2.9). On the other hand, if an area of $10 \times 10 \mu \mathrm{m}^{2}$ is milled with a current of $100 \mathrm{pA}$, the impingement rate of ions is $6 \times 10^{14} \mathrm{~cm}^{-2} \mathrm{~s}^{-1}$. Thus the milling rate may be seriously affected. Moreover, when an atmosphere of water is deliberately introduced, the milling rate of reactive materials such as $\mathrm{Si}$ is decreased. ${ }^{137}$ In fact, the negative milling yield of $\mathrm{Al}$ by $\mathrm{Si}$ ions (see Table $\mathrm{V})$ may be partly due to the fact that $\mathrm{Al}$ rapidly reacts with the ambient gas. In addition there is some evidence that surface diffusion of adsorbates such as hydrocarbons may play a role at low doses by causing deposition of material. ${ }^{138}$

(3) The angle of ion incidence on the surface affects the sputter yield. Thus a surface that has some topography may mill at a different rate than a flat surface. The increase in milling yield can be several-fold in going from normal toward grazing incidence as shown in Fig. 15. However, at grazing incidence the yield again falls. This is due to the fact that some of the incident ions are reflected. This can lead to unwanted milling due to these reflected ions. This is seen in Fig. 16(a) where we see trenches along the edge of the bottom of a pit which we would have expected to have a flat bottom.

(4) The yield may depend on the focused ion beam scan rate. If the beam is scanned slowly and the thickness of material removed per scan is comparable to the beam diameter, then locally under the beam the angle of incidence will not be normal. This leads to an enhanced milling rate and, in addition, ions are reflected and may result in additional milling. ${ }^{35,139-141}$. This effect may increase the observed sputter yield by as much as a factor of 2 .

(5) The sputtered material may redeposit on the substrate. 

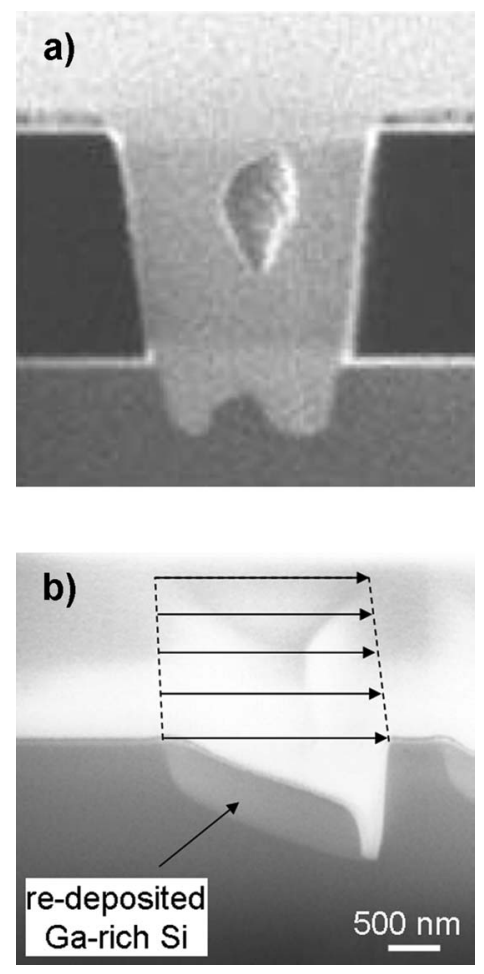

FIG. 16. (a) Milling with a stationary FIB. Self-focus effect due to reflection of ions at the steep sidewalls. The reflected ions mill at the periphery of the pit bottom and temporarily form a nonplanar bottom (from Ref. 35). (b) Redeposition during FIB milling of silicon with Ga ions. The milling was performed by a series of single scans from left to right. The increasing depth is due to the angular dependence of the sputter yield (adapted from Ref. 28).

The sputtered atoms have been shown to be emitted from the surface in a cosine squared distribution, which is maximum normal to the surface and going to zero at grazing angles. ${ }^{28,142,143}$ If a substantial thickness is removed per scan, then redeposition may decrease the milling rate and the measured sputter yield. This is illustrated by an extreme case of redeposition in Fig. 16(b).

(6) Reaction of gallium with the substrate occurs in some materials. The effects we have discussed so far, which determined the material removal rate, do not specifically depend on the fact that $\mathrm{Ga}$ ions are used. Approximately the same results would be obtained, for example, with krypton ions that have close to the same mass. This is not the case with some III-V compounds such as GaAs, GaSb, or GaN or antimony. Anyone who has milled GaAs with Ga ions has seen droplet formation on the surface. In focused ion beam irradiation of $\mathrm{GaSb}$, microcavities and filaments are observed, ${ }^{144}$ nanoblisters and agglomeration of Ga are observed in the irradiation of $\mathrm{GaN},{ }^{145}$ while the irradiation of antimony produces a porous nanowire network. ${ }^{146}$ These effects are complicated and poorly understood. We will not discuss them further here, even though they may be important and may be exploited in interesting ways in the future.

(7) Ion-surface interaction can be expected to be temperature dependent. For example, raising the temperature of Si to $400{ }^{\circ} \mathrm{C}$ greatly reduces channeling effects in implantation. ${ }^{147}$ One would expect substrate temperature to play a role in FIB milling. Under some circumstances, e.g., low thermal conductivity samples, high beam currents, and slow scans, the beam may heat the sample locally (see Sec. II F). The milling rate of crystalline $\mathrm{Si}$ has been observed to decrease by $40 \%$ when the temperature of the substrate is increased to $350{ }^{\circ} \mathrm{C}$, while the milling rate of amorphous $\mathrm{Si}$ is unchanged. No material swelling due to amorphization at low overall doses was observed at a target temperature of $350{ }^{\circ} \mathrm{C}$. At room temperature this swelling is observed. A dose rate dependent effect, namely, beam induced recrystallization that depends on temperature, may affect milling rates. ${ }^{148}$

To avoid the effects in (4) and (5) in either measuring the normal incidence milling yield or in milling a sample to a desired predictable depth, the scan speed of the beam should be high enough so that these effects do not come into play. To avoid reaction with residual gas, low current scans over large areas should not be used. A way to mitigate the roughness of the surface of a focused ion beam milled polycrystalline metal has been demonstrated. ${ }^{149}$ The surface to be milled was first patterned into a dense array of cones, called the egg crate pattern. As a result in further milling, there was a large variation of angles of incidence so channeling effects were averaged out and pits could be milled in copper with a flat bottom. In some cases a reactive gas can be used. We will discuss this later in Sec. V D 2.

Apart from the complications listed, the milling yield, of course, depends in general on the substrate, the ion mass, and the ion energy. These effects have been studied in detail experimentally and theoretically. ${ }^{150}$ The milling yield increases with energy and, depending on the ion/substrate combination, peaks in the 50-150 keV range.

Since $\mathrm{Ga}$ and $\mathrm{Kr}$ have almost the same mass, one might expect the milling yields to be the same. However, $\mathrm{Ga}$ is a solid and $\mathrm{Kr}$ is a gas. So the $\mathrm{Ga}$ that is implanted in the substrate has to be sputtered off while $\mathrm{Kr}$ may leave the surface spontaneously.

Monte Carlo and molecular dynamics approaches of FIB sputtering were reviewed in detail in Ref. 159. Semiempirical formulas of sputter yields for $\mathrm{Ar}, \mathrm{Ne}$, and $\mathrm{Xe}$ ions are given in Refs. 133, 160, and 161. For free MC programs simulating ion interactions with amorphous solids we refer to SRIM (Refs. 73 and 80) or Geant 4. ${ }^{162}$ A commercial software tool including redeposition and self-focus effects is IONSHAPER. ${ }^{163}$

In many practical FIB milling situations, one may need to sculpt a particular profile, for example, a blazed grating. The FIB is particularly well suited for this since most other micro-nanofabrication techniques remove or add material uniformly. Because of the effects discussed above, in particular, the dependence of milling rate on angle of incidence and the effect of redeposition, FIB sculpting is in many cases not straightforward and computer models of the process have been developed. ${ }^{142,143,164,165}$ 
TABLE VI. Summary of vapor pressures $\left(P_{\text {vap }}\right)$ and molecule diameters $\left(\delta_{m}\right)$ of selected precursors. The mean free path $\lambda$ was calculated at vapor pressure according to Eq. (2.8a). The monolayer density was calculated according to $n_{0}=1.154 \delta_{m}^{-2}$. The Knudsen number $(\mathrm{Kn}=\lambda /$ diameter $)$ was calculated for a typical tube diameter of $0.6 \mathrm{~mm}$.

\begin{tabular}{lcccccc}
\hline \hline Precursor & $P_{\text {vap }}(\mathrm{Pa}) / T$ & $\delta_{m}(\AA)$ & $n_{0}\left(\mathrm{~nm}^{-2}\right)$ & $\lambda(\mu \mathrm{m})$ & \multicolumn{1}{c}{$\mathrm{Kn}$} & $P_{\text {vap }}$ Ref. \\
\hline $\mathrm{Cu}(\mathrm{hfa})_{2}$ & $0.4 / 25^{\circ} \mathrm{C}$ & $8.0^{\mathrm{a}}$ & 1.0 & 3614 & 6 & 168 \\
$\mathrm{Me}{ }_{2} \mathrm{Au}(\mathrm{tfa})$ & $7.3 / 23^{\circ} \mathrm{C}$ & $3.5^{\mathrm{b}}$ & 9.6 & 1067 & 1.8 & 169 \\
$\mathrm{Mo}(\mathrm{CO})_{6}$ & $14.9 / 23^{\circ} \mathrm{C}$ & $3.3^{\mathrm{b}}$ & 10.9 & 589 & 1 & 169 \\
{$\left[\left(\mathrm{PF}_{3}\right)_{2} \mathrm{RhCl}\right]_{2}$} & $7.5 / 23^{\circ} \mathrm{C}$ & $5.7^{\mathrm{b}}$ & 3.6 & 385 & 0.6 & 169 \\
$\mathrm{Co}_{2}(\mathrm{CO})_{8}$ & $13.4 / 27^{\circ} \mathrm{C}$ & $7.0^{\mathrm{b}}$ & 2.4 & 139 & 0.2 & 170 \\
$\mathrm{MeCpPt}(\mathrm{Me})_{3}$ & 7.7 & $7.8^{\mathrm{b}}$ & 1.9 & 199 & 1.3 & 171 \\
$(\mathrm{hfa}) \mathrm{Cu}-\mathrm{VTMS}$ & $10 / 25^{\circ} \mathrm{C}$ & $8.6^{\mathrm{a}}$ & 1.6 & 126 & 0.2 & 172 \\
$\mathrm{H}_{2} \mathrm{O}$ & $2330 / 20^{\circ} \mathrm{C}$ & $3.5^{\mathrm{a}}$ & 9.4 & 3.3 & 0.0054 & 173 \\
$\mathrm{TEOS}$ & $172 / 25^{\circ} \mathrm{C}$ & $8.1^{\mathrm{a}}$ & 1.8 & 8.3 & 0.014 & 174 \\
$\mathrm{TMOS}$ & $23097 / 20^{\circ} \mathrm{C}$ & $7.0^{\mathrm{a}}$ & 2.4 & 0.08 & 0.00014 & 175 \\
$\mathrm{O}_{2}$ & $\cdots$ & $3.7^{\mathrm{c}}$ & 8.7 & $\cdots$ & $\cdots$ & $\cdots$ \\
$\mathrm{H}_{2}$ & $\cdots$ & $2.7^{\mathrm{c}}$ & 16.1 & $\cdots$ & $\cdots$ & $\cdots$ \\
$\mathrm{XeF}_{2}$ & $507 / 25^{\circ} \mathrm{C}$ & $6.7^{\mathrm{b}}$ & 2.6 & 4.1 & 0.007 & 175 \\
$\mathrm{XeF}_{2}$ & $507 / 25^{\circ} \mathrm{C}$ & $4.5^{\mathrm{a}}$ & 5.6 & 9 & 0.015 & 175 \\
\hline \hline
\end{tabular}

${ }^{\mathrm{a}}$ From density and molar mass [Eq. (2.8b)].

${ }^{\mathrm{b}}$ Longest dimension of molecule.

${ }^{\mathrm{c}}$ Apparent diameters from Ref. 176. Note the different diameters for $\mathrm{XeF}_{2}$ obtained from the different methods.

\section{Impinging precursor flux}

The supply of molecules and the knowledge of their distribution and supply density at the place of irradiation are obviously of utmost importance in gas-assisted FEB and FIB processing. Before we enter into the quantitative description of the molecule flux delivered by tube-based gas injection systems, we would like to point out that care should be taken to minimize molecule reactions with the walls of the precursor reservoir and the tube system. In the specific case of $\mathrm{Fe}(\mathrm{CO})_{5}$ under ultrahigh vacuum conditions, such wall reactions were observed and led to a high carbon monoxide concentration in the flux since the iron was bound to the walls. ${ }^{166}$ Evidently, any deposit composition will depend on the impinging flux composition and, furthermore, the uptake of precursor molecules at the tube walls will change the impinging flux distribution.

The mathematical framework necessary to adequately describe the gas flow depends on the Knudsen number, which is the ratio of the mean free path (MFP) between molecule collisions and a characteristic dimension of the gas injection system under consideration. The mean free path is given by

$$
\lambda=\frac{k T}{\sqrt{2} \pi \delta_{m}^{2} P},
$$

where $P$ is the pressure, $T$ is the absolute temperature, and $k$ is the Boltzmann constant. Assuming that molecules are closely packed as in a face-centered cubic lattice, the molecule diameter $\delta_{m}$ can be estimated from ${ }^{167}$

$$
\delta_{m} \cong 1.122\left(\frac{M}{\rho N_{A}}\right)^{1 / 3},
$$

with $\rho$ as the density of the bulk or liquid and $M$ as the molar mass of the molecule. $N_{A}=6.02 \times 10^{23} \mathrm{~mol}^{-1}$ is Avogadro's constant. Another approach uses the maximum bond length of the molecule from crystallographic data. Both approaches can differ within several tens of percent for the same molecule according to its geometric configuration (planar, linear, etc.). Often the gas injection system has tube geometry and the characteristic dimension is its diameter. Knudsen numbers can thus be calculated and examples are given in Table VI.

Molecular flow where no intramolecular collisions occur is obtained when the MFP is larger than the diameter of the gas injection tube, i.e., the Knudsen number $\mathrm{Kn}>1$. A transient flow regime, where intramolecular collisions occur, is established for $0.1<\mathrm{Kn}<1$. Laminar or viscous flow holds for $\mathrm{Kn}<0.1$. Since vapor pressures of molecules vary over order of magnitudes (see Table VI), all flow regimes can be established; each requiring a specific mathematical framework. However, the situation simplifies due to the fact that a pressure gradient installs along the gas injection tube. This means for a molecule coming from the precursor reservoir and approaching the tube exit that its MFP becomes steadily (and considerably) larger. This is due to expansion into the microscope chamber, which is constantly pumped down to a given background pressure generally smaller than $10^{-4} \mathrm{mbar}$ (corresponding to roughly $\mathrm{MFP}=10 \mathrm{~cm}$ ). Thus the local pressure at tube exits and above the substrate can mostly be considered as molecular and we can use the framework of rarefied kinetic gas theory to derive some useful relations in the next sections.

\section{Molecule flux in chambers and at tube exit}

The uniform molecular flux (in units of molecules per unit area and unit time) incident on a surface placed in a chamber with pressure $P$ is, according to the kinetic theory of gases, 
a)

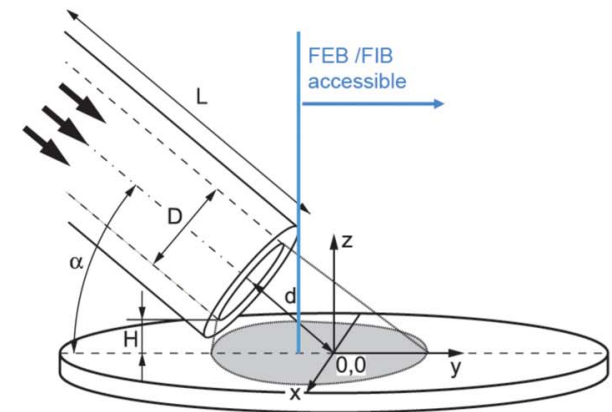

b)

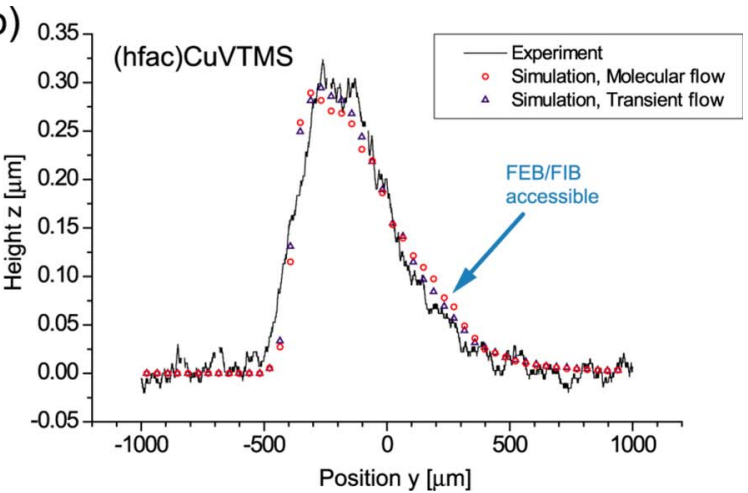

FIG. 17. (a) Tube-based precursor supply. The flux distribution impinging on the substrate depends on the supply angle $a$, the distance $d$, the height $H$, and the inner diameter $D$ of the tube. The access region for FIB/FEB is indicated. (b) Measurement and simulation of impinging precursor distribution. Tube inclination of $60^{\circ}$ and inner tube diameter $0.5 \mathrm{~mm}$. The precursor flux peak is covered by the tube geometry (from Ref. 179).

$$
J=P N_{A}(2 \pi M R T)^{-1 / 2},
$$

with $N_{A}$ as the Avogadro constant, $R=8.314 \mathrm{~J} \mathrm{~mol}^{-1} \mathrm{~K}^{-1}$ the universal gas constant, $T$ the absolute temperature, and $M$ the molar mass of the gas molecule. Equation (2.9) is equivalent to the well known expression for Knudsen effusion. As an example we calculate the flux of water molecules in an environmental SEM, $J=7.2 \times 10^{6} \mathrm{~nm}^{-2} \mathrm{~s}^{-1}$ at $P=2 \mathrm{mbar}$ (vapor pressure of water at $-13{ }^{\circ} \mathrm{C}$ ) and $J=7.2 \mathrm{~nm}^{-2} \mathrm{~s}^{-1}$ at $P$ $=2 \times 10^{-6} \mathrm{mbar}$ (typical background pressure of SEM); in units of monolayer per second $J=7.6 \times 10^{5} \mathrm{ML} / \mathrm{s}$ and $0.76 \mathrm{ML} / \mathrm{s}$, respectively. This result is obtained by dividing $J$ by the monolayer density $n_{0}$ of water from Table VI.

The molecule flux at the tube exits is easily measurable via changes in evaporated mass $\Delta m$ (solid precursor) or volume $\Delta V$ (liquid precursor) during the injection period $\Delta t$,

$$
J=\frac{\Delta m}{\Delta t} \frac{N_{A}}{M} \frac{1}{A_{\text {exit }}},
$$

where $A_{\text {exit }}$ is the tube exit surface. Injecting molecules changes the chamber pressure. Using the pump speed $S$ (volume per unit time) and assuming zero loss due to condensation at the chamber wall, the molecule flux at the tube exit can be calculated as

$$
J=P_{\text {chamber }} N_{A} \frac{1}{A_{\text {exit }}} \frac{S}{R T} .
$$

However, the pump speed $S$ should be known for the specific gas molecule and the setup, which includes pressure losses due to protection grids and pipes connecting the pump with the chamber. Compared to Eq. (2.10), Eq. (2.11) should be considered as rough order of magnitude estimation. Calibrated mass flow meters allow adjusting the molecule flux for gaseous precursors.

An analytical estimate for the molecular flux at the exit of straight long pipes with uniform circular cross section is given by ${ }^{177}$

$$
J_{\text {tube }}=P N_{A}(2 \pi M R T)^{-1 / 2} \frac{4}{3} \frac{D}{L},
$$

where $D$ is the tube diameter and $L$ is the tube length. For $D / L<1 / 20$ this estimate is better than $5 \%$ precise compared to exact calculations by Ref. 178 . The factor $4 D /(3 L)$ is the transmission probability of long tubes and defines the pressure drop between the tube entry at pressure $P$ and the tube exit. For $D / L=1 / 10$ the theoretical pressure drop becomes $11 \%{ }^{178}$ In real gas injection systems the geometry is often curved and it is unknown whether the precursor vapor pressure is constant within the reservoir and maintained until the tube entry. For the same $D / L=1 / 10$ ratio and elbow reservoir connection pipes, we measured pressure drops of $3 \%-8 \%$ for different metal organic precursors (back calculated from mass loss measurements). Nevertheless, Eq. (2.12) illustrates the scaling of precursor exit flux with diameter and length of straight tube-based gas injection systems at molecular flow conditions.

The average precursor flux impinging on the substrate as a function of microtube-substrate distance $[H$ in Fig. 17(a)] was measured using a stagnation tube.$^{58}$ The impinging flux decayed roughly inversely with the height of the tube that was placed over the substrate.

\section{Spatial distribution of molecule flux}

The impinging precursor flux distributions of FEB and FIB relevant gas injection systems and precursors were experimentally determined from deposit shapes obtained by using a heating stage in an electron microscope, which allowed us to thermally decompose all impinging precursor molecules on the substrate. ${ }^{179}$ Since the growth rate is proportional to the impinging flux, the deposit shape directly measures the spatial distribution of the incident molecules. Good agreement was found with MC-simulated molecule distributions [see Fig. 17(b)]. It also shows that the spatial distribution within a $100 \mu \mathrm{m}^{2}$ writing field cannot be necessarily 

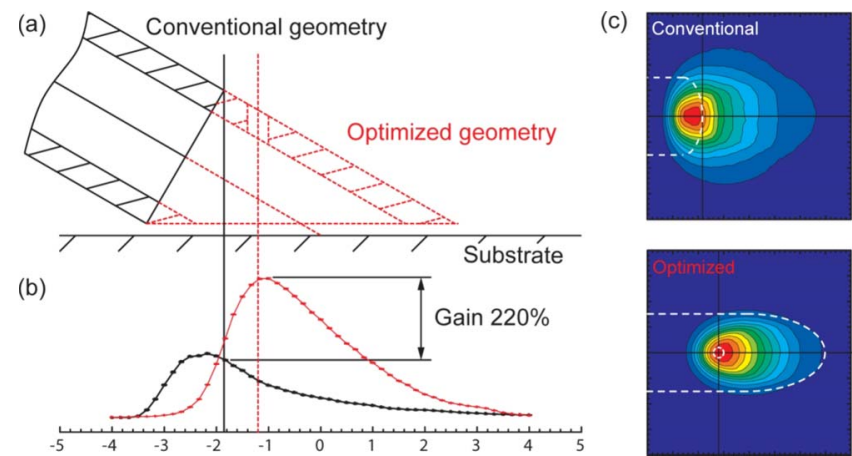

FIG. 18. MC-simulated molecule flux impinging on the substrate for different tube geometries. (a) Cross section of tube geometries along the tube axis. (b) Molecule flux distribution taken along the tube axis. The $x$-axis is in units of the inner tube radius. (c) Molecule flux distribution from top view. White dashed lines represent the tube periphery. The color code is red (highest flux) to blue (lowest flux=0) (from Ref. 181)

considered as constant. The distribution of water molecules was measured on a cryocooled substrate inside a dual beam system. $^{180}$

To obtain high and localized molecule flux, the tube should be as close as possible to the substrate. For a given height of the tube above the substrate, the angle between the injection tube and the substrate strongly determines the maximum precursor flux on the substrate surface accessible for the FEB and FIB. Tube angles around $30^{\circ}$ give a good compromise between FEB and FIB access and large flux. Smaller tube angles distribute the molecules over a larger area and result in smaller fluxes, whereas larger tube angles prohibit the FEB and FIB access to the peak of the flux distribution. Introducing an additional pinhole into the tube wall allows increasing the molecule flux by a factor of 2 and permits access of the FEB and FIB to the peak flux value (see Fig. 18).

\section{Shadow effects}

Shadow effects arise inevitably when the precursor flux is directed and the deposit or etch features become a highaspect ratio. The deposition rate is higher when the electron beam is scanned toward the flux, and lower when scanned with the flux as illustrated in Fig. 19. The contributions of precursor molecules in shadowed regions are supposed to mainly arise from local molecule gas phase collisions, from molecule desorption from surfaces next to the growing deposit, and, to a minor extent, from surface diffusion. ${ }^{182}$

\section{Gas phase related processes}

Within the gas phase volume above the substrate, collisions between molecules with incident electrons and ions as well as emitted electron and sputtered atoms occur, which leads to scattering, ionization, and dissociation [see Fig. 20(a)]. Scattering in the gas phase above the substrate becomes significant when the mean free path of electrons or ions $\lambda_{e, i}$ in a gas with density $\rho=M^{*} P /(R T)$,

$$
\lambda_{e}=M /\left(N_{A} \rho \sigma\right),
$$

becomes smaller than the distance traversed by the primary beam in the gas phase. For simulation purposes the scattering cross sections can be taken as for solids (but much diluted). ${ }^{45}$ The electron mean free path scales inversely with gas pressure, which can range from $10^{-6}$ mbar (background pressure) to about 1 mbar (local injection gas pressure or background pressure in environmental and variable pressure microscopes). The fraction of gas phase scattered incident electrons or ions becomes

$$
N_{S}=1-\exp \left(-L / \lambda_{e}\right),
$$

where $L$ is the length traversed by the beam in the gas phase. For tube-based gas injection systems, the local pressure must be taken into account [see Fig. 20(a)].
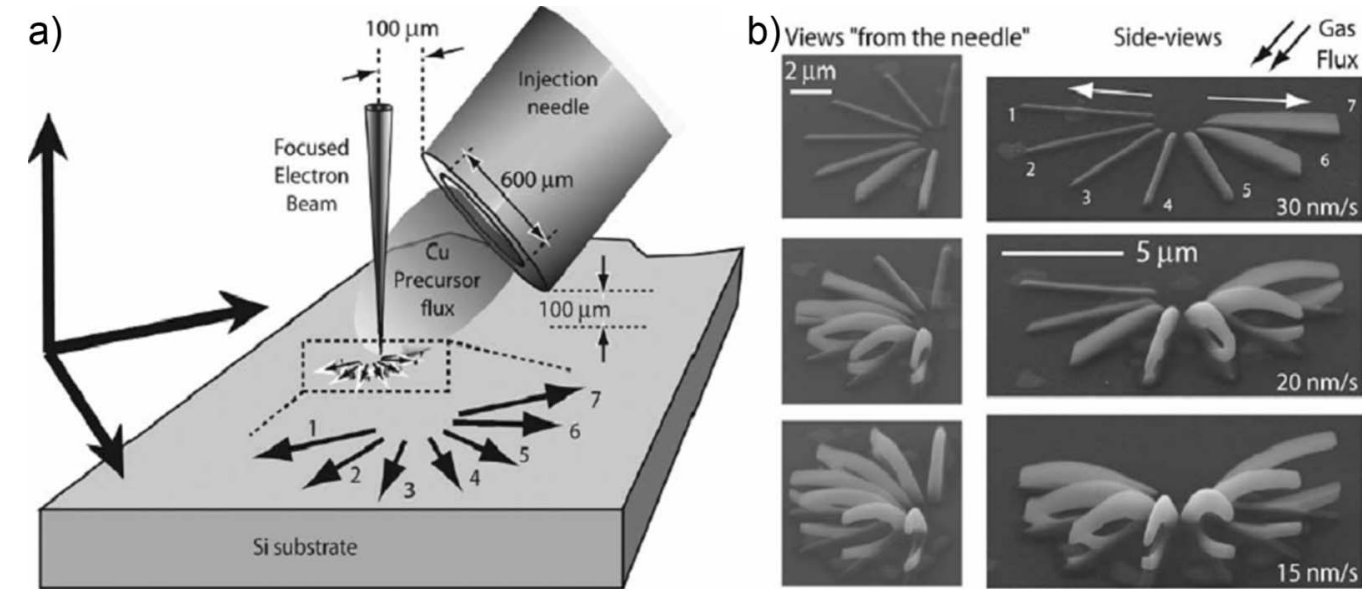

FIG. 19. Lines deposited with (hfa)Cu-VTMS precursor and varying scan direction with respect to the gas flux. Note that the variation of gas pressure over the scanned field is negligible. Lines 1-7 are scanned with the same speed. Line 1 is scanned away from the gas feed tube and line 7 is scanned toward the gas feed tube. (a) Schematics of the experimental setup and example of writing sequence. (b) SEM micrographs, tilted views (70 $\left.{ }^{\circ}\right)$ of the resulting structures, at three different scan speeds. Left: front views; right: side views (from Ref. 182). 


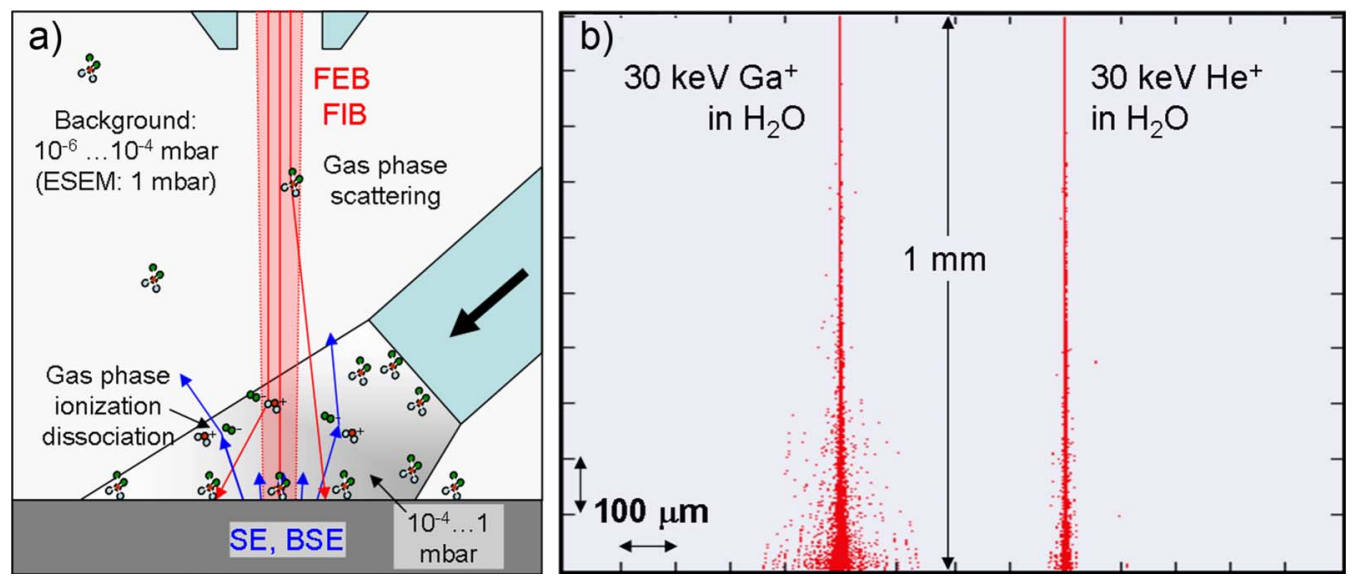

FIG. 20. (a) Gas phase effects. Typically the incident beam traverses about $5-10 \mathrm{~mm}$ gas phase at background pressure and roughly $0.5-1 \mathrm{~mm}$ at locally increased pressure before impinging on the substrate. (b) MC-simulated trajectories (SRIM) of incident ions traversing $1 \mathrm{~mm}$ water vapor at $1.4 \mathrm{mbar}$.

A Gaussian fit was proposed to the measured distribution of scattered electrons under certain conditions. ${ }^{183} \mathrm{MC}$ simulations show that scattered incident electrons and ions have a non-Gaussian skirt distribution (see Appendix C).

Table VII and Fig. 20(b) show that for gas phase scattered incident electrons and ions, the skirt full widths comprising $50 \%$ of all electrons are in the micrometer range. Since the flux distribution of the scattered electrons and ions scales with the inverse square of the full widths, a low background flux is obtained, meaning that the resolution of the primary beam is not lost even at relatively high collision percentages. Part of the gas phase collisions can produce ionized molecules at an amount given by the ionization cross section and its energy dependence (compare to Sec. II B 1). However, it is the "fast" emitted secondary electrons that can ionize molecules with the highest efficiency in the gas phase just above the substrate since the ionization cross section peak is at around $100 \mathrm{eV}$. The same holds for the other low-energy dissociation mechanisms discussed above. At $\mathrm{keV}$ incident energies the efficiency for all these mechanisms is considerably lower. This is why gas phase initiated reactions within the incident charged particle beam and within the backscattered electron "cloud" might be mostly neglected. This is in contrast to some local photon (laser) beam deposition experiments. ${ }^{184}$

However, the role of secondary electron emission on gas phase ionization, or dissociation in general, has not yet been studied systematically in this context to our knowledge. A better resolution in focused electron beam induced deposition of dots with reduced or even closed precursor supply was noticed, ${ }^{185,186}$ which might hint to an additional supply mechanism mediated by a secondary electron-gas phase reaction. A way to distinguish between surface or gas phase controlled deposition (etching) is to change the substrate temperature. For surface controlled dissociation a decrease in process rate with increasing temperature is detected, which allows us to determine the desorption energy (see Sec. III B). In the case of gas phase determined molecule dissociation, substrate heating should result in negligible changes of process rates.

\section{E. Precursor migration \\ 1. Adsorption and desorption}

A molecule may bind at the surface as a chemisorbed or as a physisorbed species. The reversible physisorption is often a weak, dipole induced dipole interaction, while chemisorption implies the formation of a chemical bond to the surface and is typically about $1 \mathrm{eV}$ (about $100 \mathrm{~kJ} / \mathrm{mol}$ ) strong and nonreversible (except when the reaction product is volatile $=$ spontaneous etching). Typical adsorption energies for a physisorption are $0.1 \mathrm{eV}$ and up to $0.5 \mathrm{eV}$ for larger organic molecules. They are comparable to heats of evaporation/sublimation or even larger when a long range image charge potential on a conductive surface is involved. Heats of evaporation/sublimation of numerous precursors are summarized in the tables in Sec. V. Chemisorption is unwanted since deposition and etching would occur nonlocalized on the entire gas-exposed substrate area instead of just inside the focused spot of the electron or ion beam.

The dynamic equilibrium between physisorption (adsorption) and desorption of gas molecules leads to an average constant molecule coverage on the surface. Measuring the

TABLE VII. Skirt MC simulations [Mocasim (Ref. 81)] of incident electrons (zero diameter beam, 30 and $3 \mathrm{keV}$ ) traversing $1 \mathrm{~mm}$ water vapor at a pressure of $1.4 \mathrm{mbar}$ (density: $\left.1 \times 10^{-6} \mathrm{~g} / \mathrm{cm}^{3}\right)$. Mean free electron paths $\left(\lambda_{e}\right)$, the fraction of scattered incident primary beam electrons $\left(N_{S}\right)$, and skirt full widths comprising $10 \%$ and $50 \%$ of all scattered electrons are given.

\begin{tabular}{lcc}
\hline \hline $\begin{array}{l}\text { Molecule } \\
\text { (energy) }\end{array}$ & $\begin{array}{c}\mathrm{H}_{2} \mathrm{O} \\
(30 \mathrm{keV})\end{array}$ & $\begin{array}{c}\mathrm{H}_{2} \mathrm{O} \\
(3 \mathrm{keV})\end{array}$ \\
\hline$\lambda_{e}(\mathrm{~mm})$ & 23.9 & 2.8 \\
$N_{S}(\%)$ & 4.2 & 30 \\
FW10 $(\mu \mathrm{m})$ & 0.07 & 0.6 \\
FW50 $(\mu \mathrm{m})$ & 1.8 & 9.2 \\
\hline \hline
\end{tabular}


coverage at constant temperature for varying gas pressures gives the so-called adsorption isotherms, which are almost exclusively reported for ultrahigh vacuum conditions, solidvapor equilibrium, and well defined crystallographic oriented surfaces of single crystals. All these conditions are violated by the nature of the FEB and FIB processes. The pragmatic approach in FEB and FIB modeling assumes a nondissociative Langmuir adsorption isotherm according to which the molecule coverage $\theta=n / n_{0}$ depends on the impinging flux $J$ (or pressure),

$$
\frac{n}{n_{0}}=\theta=\frac{b J}{1+b J}=\frac{s J \tau / n_{0}}{1+s J \tau / n_{0}},
$$

where $b$ is a (temperature dependent) thermodynamic parameter $b=s \tau / n_{0}, \tau$ is the residence time, $s$ is the sticking probability, and $J$ is the impinging precursor flux. Equation (2.14a) is obtained when setting the diffusion and decomposition term to zero in Eq. (2.2). The maximum monolayer coverage $n_{0}$ is given by the number of available adsorption sites but often taken as the inverse of the molecule size (see Table VI) since the physisorption potential is relatively insensitive to the site or orientation of the adsorbate. Of note is that this adsorption type accounts for already occupied adsorption sites by the gas molecules and limits the maximum surface density to $n_{0}$. This property of Langmuir adsorption is meaningful in view of the high volatility of the precursor molecules used. Multilayer adsorption (condensation) is not covered with this adsorption isotherm but also not expected if the substrate is not excessively cooled with respect to the precursor reservoir. ${ }^{187,188}$ Multilayer condensation starts when the impinging gas pressure becomes larger than the vapor pressure of the precursor molecule on the cooled substrate. Under this condition no steady state surface concentration will be achieved but a constantly growing film of condensed precursor. When now irradiated the situation is similar to resist exposure, i.e., lithography (see also Sec. V E).

Furthermore, the Langmuir isotherm states that adsorption is reversible, i.e., all molecules desorb in average after the residence time $\tau$. However, in the few measurements reported the formation of an initial chemisorbed monolayer is found: for $\mathrm{Me}_{2}-\mathrm{Au}(\mathrm{tfa})$ on clean $\mathrm{Si}$ wafers, ${ }^{188}$ for $(\mathrm{hfa}) \mathrm{Cu}-$ VTMS on $\mathrm{Si}(111),{ }^{111}$ and tetraethyllead on sapphire. ${ }^{184}$ Subsequent adsorption onto the chemisorbed precursor molecules was reversible so that the Langmuir adsorption isotherm can be applied. The temperature dependence of the residence time is given by the well known relation $\tau(T)$ $=\tau_{0} \exp \left(E_{\mathrm{des}} / k T\right)$, with $E_{\mathrm{des}}$ as the activation energy for desorption. The attempt periods $\tau_{0} \approx 10^{-12} \mathrm{~s}$ obtained from classical transition state theory ${ }^{189}$ do not apply for the molecules used in gas-assisted beam processing (see Sec. III B).

The residence time and the sticking probability of molecules on irradiated materials (and deposits) are generally unknown parameters. In Secs. III B and III D we discuss how the residence time can be determined from the FEB and FIB deposition or etch experiments. A general rule was found experimentally: when the precursor molecules are po- lar or easily polarizable, the adhesion forces are high, i.e., the average residence time $\tau$ is large, and the sticking probability, too. ${ }^{190}$ Generally, ions or radicals do not desorb readily from uncharged surfaces. Easily desorbing units are intact uncharged and nonpolar molecules.

As we mentioned at the beginning of this section, the nondissociative Langmuir adsorption is a pragmatic approach for gas-assisted FEB and FIB, which holds true for simple molecules. For large molecules this is not necessarily the case. The above mentioned (hfa)Cu-VTMS molecule, for example, shows dissociative adsorption and desorption behavior. ${ }^{191}$ As a result the molecule coverage $\theta$ can be described by the dissociative Langmuir isotherm

$$
\theta=\frac{\sqrt{b J}}{1+\sqrt{b J}}
$$

Modeling in the field of gas-assisted FEB and FIB processing including such adsorption behavior or even more complicated ones has not yet been performed. For instance, the most general isotherm is the Brunauer-Emett-Teller isotherm, ${ }^{192}$ which can account for multilayer adsorption; however, it requires the knowledge of further interaction parameters, which are a priori unknown for the molecules used in FEB and FIB gas-assisted processing.

\section{Surface diffusion}

Generally in discussing gas-assisted focused ion beam or electron beam processes, one frequently assumes that only adsorption and desorption play a role in the surface coverage by the reacting gas. However, from experiments there is a reason to believe that surface diffusion can also play a significant role. Data on surface diffusion obtained from focused beam induced deposition and etching experiments will be discussed in Sec. III D. Independent surface diffusion measurements at the microscopic scale rely, for instance, on scanning tunneling microscopy or cold field emission microscopy. In the latter method the field emitter surface is displayed on a monitor and the motion of adsorbates on the emitter is viewed through a contrast in emission due to the change in work function. For a review on more measurement methods, see Ref. 193. In surface diffusion of adsorbed gases on metal surfaces, it is stated that diffusion at low coverages can be considered as random motion, whereas at large coverages lateral interactions with neighboring adsorbates make diffusion a collective process. ${ }^{194}$ For instance, the diffusion coefficient of $n-\mathrm{C}_{4} \mathrm{H}_{10}$ on single crystal ruthenium at a temperature of $125 \mathrm{~K}$ varied from $D=3 \times 10^{-10}$ to 2 $\times 10^{-7} \mathrm{~cm}^{2} / \mathrm{s}$ for coverages of 0.2 and 1 , respectively. The mean diffusion path the molecule can travel during a given time $t$ is roughly $(D t)^{1 / 2}$. For the above molecule the diffusion paths traveled within $1 \mathrm{~s}$ are $170 \mathrm{~nm}$ and $4.5 \mu \mathrm{m}$, respectively.

Surface diffusion is a thermally activated process and the diffusion coefficient is written as $D(T)=D_{0} \exp \left(-E_{\text {diff }} / k T\right)$, with $D_{0}$ in the order of $10^{-2}-10^{-3} \mathrm{~cm}^{2} / \mathrm{s}$. There is an often quoted similarity between molecule desorption and diffusion: desorption is the rupture of a bond between an adsorbed 
molecule and the surface, and migration is the rupture of the same bond with simultaneous formation of an analogous bond with an adjacent adsorption site. From this consideration a general relation between the activation energies for diffusion and adsorption was experimentally found: $E_{\mathrm{des}} / E_{\mathrm{diff}}=3-10$. The major differences to surface diffusion in gas-assisted FEB and FIB processing is that molecules are physisorbed and that the underlying (deposited) material has no defined periodic surface potential. In fact, the molecules are not supposed to form a chemical bond with the substrate; otherwise the local beam resolution of the whole process would be lost. As a consequence, relations and values established for $D_{0}$ and $E_{\text {des }} / E_{\text {diff }}$ from well defined surfaces and ultrahigh vacuum conditions are not straightforwardly applicable to gas-assisted FEB and FIB processing and need to be proven.

An example of the influence of how hydrophobicity can influence diffusion is given in Ref. 195. The surface diffusion coefficient of the polycyclic aromatic hydrocarbon naphthacene $\mathrm{C}_{18} \mathrm{H}_{12}$ adsorbed on silica gel was determined to be $D=(2.3-2.8) \times 10^{-10} \mathrm{~cm}^{2} / \mathrm{s}$. There was no change in surface diffusion between dry and $2 \%$ water covered surfaces. However, changing the surface to hydrophobic using the saturated fatty acid $\mathrm{CH}_{3}\left(\mathrm{CH}_{2}\right)_{12} \mathrm{COOH}$ (myristic acid) increased surface diffusion by a factor of 10 .

\section{Electrostatic field mediated phenomena}

Due to local electrical charging of insulating material irradiated with focused ion or electron beams, considerable electrostatic fields can be generated. Visualization of electrical fields on planar quartz was achieved by toner powder ${ }^{196}$ and by objective lens defocus in a TEM, as shown in Fig. 21(b) for high-aspect-ratio carbon filaments. The exponential decay of such fields due to electron-hole pair combination can last over several days in the case of quartz. Various electrostatic field enhanced phenomena are reported.

(1) Coulomb attraction of ionized molecules: As discussed in Sec. II D 4, gas phase collisions of electrons with molecules produce ionized fragments, which in turn can be attracted in the electrostatic fields generated locally by the impinging beam on the substrate. In fact, this phenomenon is widely used for charge neutralization with deliberately introduced $\mathrm{H}_{2} \mathrm{O}$ molecules when observing insulating samples.

(2) Polarization of molecules: Molecules can be polarized in strong fields and are consequently attracted along the field gradient. Accordingly, during FEB or FIB deposition and etching, the local precursor molecule density can be drastically changed in the irradiated region: filamentlike ramified deposits due to attracted ionized or polarized molecules by the local electrostatic field generated at the end of contamination deposits $\left(\approx 10^{6}-10^{7} \mathrm{~V} / \mathrm{m}\right)$ were observed ${ }^{197}$ (see Fig. 21).

(3) Field enhanced diffusion and molecule decomposition: These phenomena are reported for STM deposition with (hfa)Cu-VTMS. ${ }^{199-201}$ The physisorbed mol-

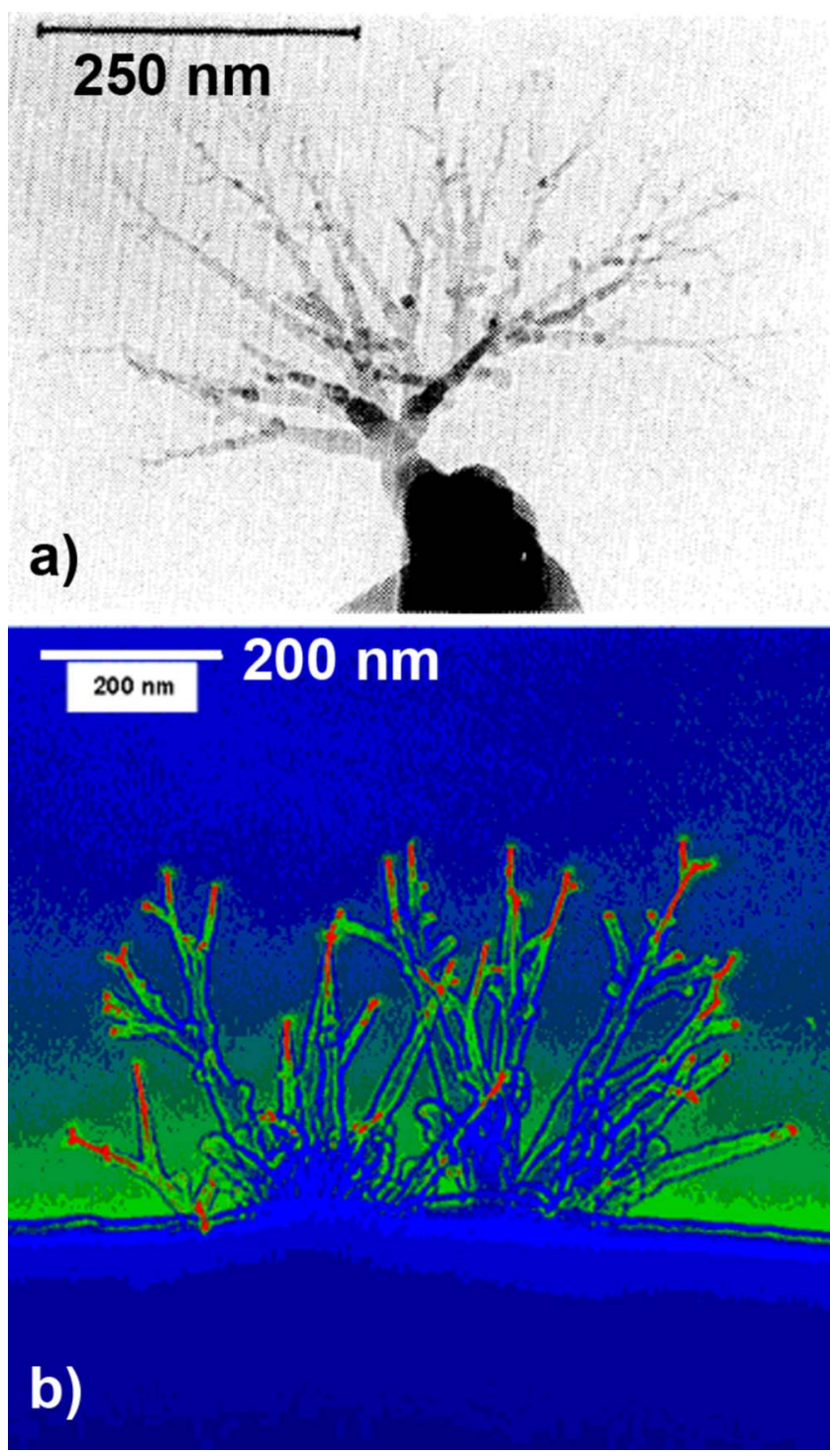

FIG. 21. (a) Example of fractal branch electron contamination deposition from background pressure hydrocarbons in the presence of large electrostatic fields. Scale bar, $250 \mathrm{~nm}$ (from Ref. 197). (b) Local electric field around amorphous carbon filaments due to irradiation, imaged by defocus of the objective lens in the TEM. Colors represent image brightness: red: high field (bright); blue: low field (dark). Local field highest at the tips: hydrocarbon molecules aggregate there (from Ref. 198).

ecules experience a force due to the electric field gradient that arises from the proximity of the STM tip to the sample. The rate of deposition was found to exceed by approximately $20-40$ times the rate of gas phase precursor molecule delivery under the tip. The critical electrostatic field for molecule dissociation was $2 \times 10^{9} \mathrm{~V} / \mathrm{m}$ and the deposit shapes were narrow conelike structures. Field-induced decomposition was also observed for STM deposition with $\mathrm{Fe}(\mathrm{CO})_{5}{ }^{202}$

(4) Solid disintegration: According to Egerton ${ }^{127}$ and Cazeaux, ${ }^{77,203}$ a typical field emission probe $(d$ $=1 \mathrm{~nm}, I=0.4 \mathrm{nA}$ ) can lead to an electric field at the edges of the illuminated area in excess of $10^{10} \mathrm{~V} / \mathrm{m}$. An estimation of the corresponding net charge showed that about $16 \%$ of the solid atoms are ionized, 
which are likely to result in disintegration by electrostatic repulsion.

The ejection of charged fragments from the surface by Coulomb forces might also play an important role in gas-assisted FEB or FIB induced processes. An example is the ejection of $\mathrm{O}^{+}$ions after dissociative decomposition of adsorbed $\mathrm{CO}$ on metal surfaces. ${ }^{115}$

The gas phase molecule ionization discussed in Sec. II D 4 and the above actions of electrostatic fields might be the reason why the smallest dot deposits were observed for a closed precursor supply ${ }^{185}$ and at lowest precursor pressure. ${ }^{186}$

\section{F. FEB/FIB heating}

Heat is generated in FEB and FIB processing by the energy loss of incident electrons or ions along their trajectories in matter. This can be modeled at steady state by a three dimensional heat source term $H(r, z)$ in the diffusion equation in cylindrical coordinates for a beam incident at the point $r=0$,

$$
\kappa\left(\partial^{2} T / \partial r^{2}+(1 / r) \partial T / \partial r+\partial^{2} T / \partial z^{2}\right)+H(r, z)=0 .
$$

Here $\kappa$ is the thermal conductivity, $T$ is the temperature, $r$ is the radial position, and $z$ is the axial position. We have assumed a stationary beam. The heat source term for a planar bulk substrate is shown in Appendix D (normalized to one primary incident electron). However, in general the beam is scanned. This would add a first order derivative of the temperature with respect to time and make the source term time dependent, $H(r, z, t)$. The major problem is that the thermal conductivity is unknown for FEB or FIB deposited material, unless pure material is deposited. Estimates of the thermal conductivity of carbon/metal nanocomposites can range between polymers $0.01 \mathrm{~W} / \mathrm{K} \mathrm{m}$ (PMMA) to metals $395 \mathrm{~W} / \mathrm{K}$ m (copper).

For planar bulk, pillar, and membrane geometry, the heat source term can be approximated and analytic estimates of the temperature rise given. This is less precise with respect to MC simulations but gives a more fundamental insight into the energy and geometry dependence of heating. The definitions of the stopping power and the electron and ion ranges involved in these formulas are summarized in Appendixes A and $\mathrm{B}$. The time between two successive electron or ion excitations should be shorter than the electron-phonon relaxation time scale around $10^{-1}-10^{-3} \mathrm{~ns}$; otherwise the temperature is not well defined. ${ }^{32}$ The time between two successive electron or ion impingements is given by $\left(e_{0} / I_{p}\right)$; thus $2 \mathrm{nA}$ beam current is needed for $0.1 \mathrm{~ns}$. This value represents an overestimate since the primary beam generates a cascade of additional secondary (charged) particles in matter.

\section{Plane bulk geometry}

The temperature increase $\Delta T$ at the center of an irradiated sample surface can be estimated by assuming that the heat of the primary beam is homogeneously dissipated inside a hemisphere of radius $R_{H}$ being equivalent to half the range of electrons $R_{E} / 2$ or the projected range of ions (see Appendix A). Heat dissipation occurs three dimensionally by heat conduction into the semi-infinite substrate. The analytic estimate is given by ${ }^{32}$

$$
\Delta T_{\text {bulk }}(r=0)=U I_{P}(1-\eta) /\left(2 \pi \kappa R_{H}\right),
$$

where $U$ is the acceleration voltage, $I_{p}$ is the charged particle current, and $\kappa$ is the thermal conductivity of the bulk substrate. The impinging beam diameter is assumed smaller than the dimension of the excitation volume. For electron irradiation $\eta$ represents the backscattered electron yield. A negligibly small part of the energy is lost to exiting SEs, $x$ rays, and Auger electrons. Increasing the incident electron energy results in a temperature decrease since the range $R_{E} \sim E^{5 / 3}$ (see Appendix A). The temperature increase using beam currents in the microampere range is in the order of $100{ }^{\circ} \mathrm{C}$ according to the substrate used. This can trigger the thermal dissociation of adsorbed molecules ${ }^{204}$ or local recrystallization, for example, in amorphous $\mathrm{TiO}_{2}$ films. ${ }^{205}$

The same formula applies for ion irradiation. With $\mathrm{Ga}$ ions at $1-50 \mathrm{keV}$ energy losses due to sputtering (mean energy around $50 \mathrm{eV}$ ), backscattered ions $(=0)$, secondary electrons (mean energy around few eV), and substrate amorphization are negligible. ${ }^{206}$ The range of Ga ions is $10-1000$ times smaller than for electrons at the same energy (see Fig. A1 in Appendix A). This means heating with FIB can become quite substantial for substrate with low heat conductivity and easily reach temperature values above the temperature of thermal dissociation of molecules. The range of $\mathrm{He}$ ions on the other hand shows approximately the same magnitude as the range of electrons.

For analytical solutions of temperature profiles in planar layered structures, we refer to Ref. 207. Measurements with nanosized thermocouples are reported in Ref. 208. The structures investigated were a $300 \mathrm{~nm}$ thick PMMA layer and a $1 \mu \mathrm{m}$ thick oxide layer on silicon. Irradiation by a $15 \mathrm{keV}$, $150 \mathrm{nA}$ electron beam of $1.7 \mu \mathrm{m}$ radius for $100 \mathrm{~ms}$ yielded a temperature rise at the resist bottom surface of approximately $18 \mathrm{~K}$. The temperature rise achieved a steady state value within $10 \mu \mathrm{s} .{ }^{209}$

\section{Pillar geometry}

The local temperature rise $\Delta T$ at the apex of a cylindrical pillar with length $L_{\text {dep }}$, diameter $D_{\text {dep }}$, and heat conductivity $\kappa_{\text {dep }}$ connected to a heat sink at fixed (room) temperature can be estimated by ${ }^{45}$

$$
\Delta T_{\text {pillar }}=\left(\frac{d E}{d s} \Delta s \frac{I_{P}}{e_{0}}\right) \frac{4 L_{\mathrm{dep}}}{\pi \kappa_{\mathrm{dep}} D_{\mathrm{dep}}^{2}} .
$$

It is assumed that the heat is absorbed at the very end of the pillar and is dissipated one dimensionally by conduction into the heat sink. The term $(d E / d s) \Delta s\left(I_{p} / e_{0}\right)$ represents the irradiation energy absorbed and is the product of the energy loss $(d E / d s)$ (see Appendix B) and the average trajectory path $\Delta s$ of electrons or ions inside the cylinder (see the inset in Fig. 22). Although this definition does not account for the change of stopping power with energy along the trajectory, it 


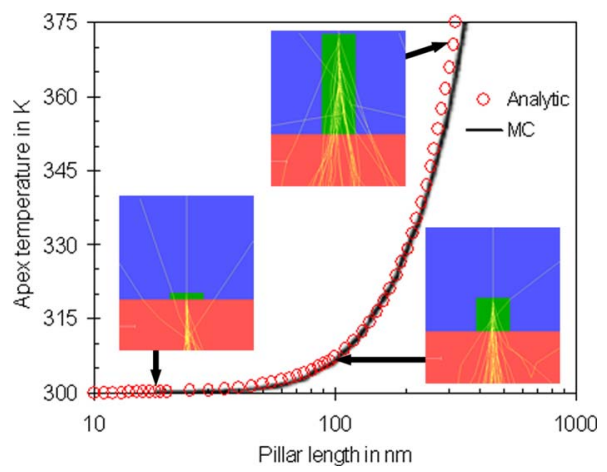

FIG. 22. Comparison of simulations from Ref. 210 with analytical estimation $\Delta T=$ const $\times L^{2}$. The $\mathrm{SiO}_{2}$ pillar with $100 \mathrm{~nm}$ diameter was irradiated with a $20 \mathrm{keV}, 500 \mathrm{pA}$ beam of electrons. Insets show 30 electron trajectories at pillar lengths of 20,100, and $300 \mathrm{~nm}$ and illustrate the shift of the interaction volume from the substrate into the pillar. (The conical apex shape is omitted.)

is a good estimate that can be readily applied to pillars and freestanding rods. Equation $(2.16 \mathrm{~b})$ can be rewritten as $\Delta T_{\text {pillar }}=$ const $\Delta s L_{\text {dep }}$. For coaxial alignment of pillar and incident beam, the average trajectory path can be taken as the observable apex cone length. If the pillar is smaller than the cone length, part of the trajectories still penetrates into the bulk substrate; the average pillar trajectory path becomes $\Delta s \approx L_{\text {dep }}$ and thus $\Delta T_{\text {pillar }} \approx$ const $L_{\text {dep }}^{2}$. The square dependence shows good agreement with a combined Monte Carlo/ finite elements simulation ${ }^{210}$ (see Fig. 22). Generally, the lower the incident electron energy, the higher is the stopping power and the smaller is the electron range (interaction volume). Thus the heat source is concentrated in the pillar apex and leads to higher temperatures for smaller incident electron and ion energies. For freestanding rods perpendicularly oriented to the incident beam, the average trajectory path becomes comparable to the rod diameter $\Delta s \approx D_{\text {dep }}$.

MC simulations performed by Weber ${ }^{211}$ conclude that no considerable temperature increase is to be expected for electron irradiation of pure metallic nanowires. Since often the deposits consist of polymerlike carbon matrix with a low heat conductance embedding the metal nanocrystals, temperatures for thermal decomposition of some precursor molecules can be reached by beam heating (see Fig. 23). Metal contents close to 100 at. \% are then obtained by thermal decomposition for $\mathrm{Co}_{2}(\mathrm{CO})_{8}$ (Ref. 104) and (hfa)Cu-VTMS. ${ }^{212}$ The metallic nanocrystals itself manifest size dependent melting behavior, which can lower the melting temperature by $600 \mathrm{~K}$ compared to bulk. ${ }^{213}$ The possibility of sintering of nanocrystals at room temperature under electron irradiation due to strong electronic excitations and plasmonic states was pointed out in Ref. 214: the plasmon energy of a nanocrystal entirely released into phonons would correspond to a temperature of several hundred degrees.

The resolution, however, is now determined by the temperature distribution, being apparently less confined than the

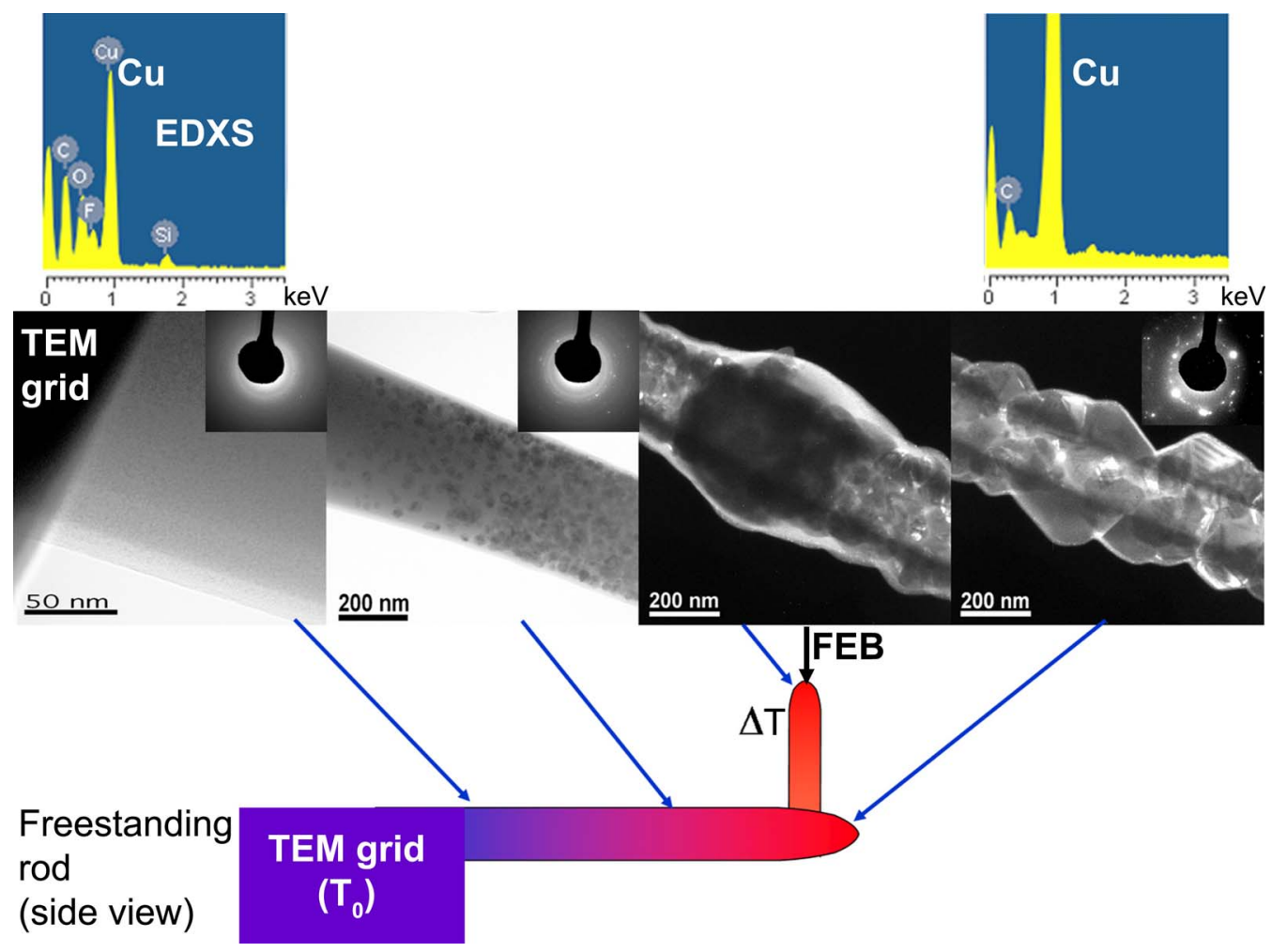

FIG. 23. Compositional and morphological changes along a horizontal freestanding rod deposit due to a temperature gradient generated while depositing a vertical tip. The precursor was (hfa)Cu-VTMS and contains the elements $\mathrm{Cu}, \mathrm{C}, \mathrm{F}, \mathrm{O}$, and $\mathrm{Si}$, which are detected by EDXS at the cold base part ( $T_{0}$ =room temperature) as a result of pure irradiative dissociation. Hydrogen is also present but cannot be detected. At the rod end Cu is predominantly detected by EDXS (at same scale with base part EDXS) while the other elements did mostly disappear due to thermal dissociation of the molecule. The metal nanocrystal size also increases from a few nanometers to several $100 \mathrm{~nm}$ with increasing temperature. 
focused beam. Cooling due to the impinging precursor flux might also play a role for inhomogeneous axial composition gradients observed in cross section investigations of pillar deposits. $^{204}$

\section{Membranes}

For membranes the temperature increase can be estimated with $^{45}$

$$
\Delta T_{m}=\left(\frac{d E}{d s} \Delta s \frac{I_{P}}{e_{0}}\right) \frac{\ln \left(2 r_{m} / r_{B}\right)}{2 \pi \kappa_{m} t_{m}},
$$

where $r_{B}$ is the beam radius and $r_{m}$ is the distance to a heat sink (e.g., the $\mathrm{Cu}$ grid for TEM supports). Heat dissipates in two dimensions inside the membrane to the heat sink. Typically, TEM membranes are very thin (around $10 \mathrm{~nm}$ ), so that electrons penetrate for incident electron energies $>1 \mathrm{keV}$, i.e., the average trajectory path becomes equal to the membrane thickness $\Delta s=t_{m}$. In this case the temperature increase becomes independent of membrane thickness and stays relatively low. However, when depositing pillars on membranes, the average trajectory path increases to the vertical size of the deposit and $\Delta s=L_{\text {dep }}$. Also the energy loss $d E / d s$ of the deposit material should be used. Since the heat dissipation in membranes is reduced to two dimensions, the temperature increase in pillars on membranes becomes more important than on bulk substrates. This can explain experimental observations that pure iron nanocrystals ${ }^{215,216}$ and gold nanocrystals $^{217}$ were found as a result of FEB induced deposition on membranes using $\mathrm{Fe}(\mathrm{CO})_{5}$ and $\mathrm{Me}_{2} \mathrm{Au}(\mathrm{acac})$ as precursors, respectively. Assuming a thermal decomposition of these molecules, temperatures of around $60{ }^{\circ} \mathrm{C}$ to $100{ }^{\circ} \mathrm{C}$ were achieved by beam heating in this configuration.

\section{FEB AND FIB CONTINUUM MODELS}

What is generally of most practical interest is how much material is removed or how much material is deposited under given circumstances. Although general trends can be predicted from FEB and FIB models, these models are still far from being quantitative since the input parameters are rarely known as was reviewed in Sec. II. Since deposition or etch rates can be measured straightforwardly, FEB and FIB continuum models generally attempt to determine the unknown parameters entering into Eqs. (2.1a), (2.1b), and (2.2). The parameters needed to be determined are the precursor molecule flux $J$, the sticking probability $s$, the volume $V$ of the deposited molecule (for etching this volume is defined by the etched material), the residence time $\tau$, the integral cross section $\sigma$, and the surface diffusion coefficient $D$ [see Fig. 24(a)]. The incident electron and ion flux distribution $f(r)$ can be measured independently (see Sec. I C). Recently, a method to independently determine the impinging precursor flux from a nozzle supply was published. ${ }^{179}$ The (maximum) monolayer density $n_{0}$ is sometimes determined experimentally or taken as the inverse of the molecule size. This makes in total six to seven independent parameters, thus requiring an equivalent number of independent experiments for their determination.
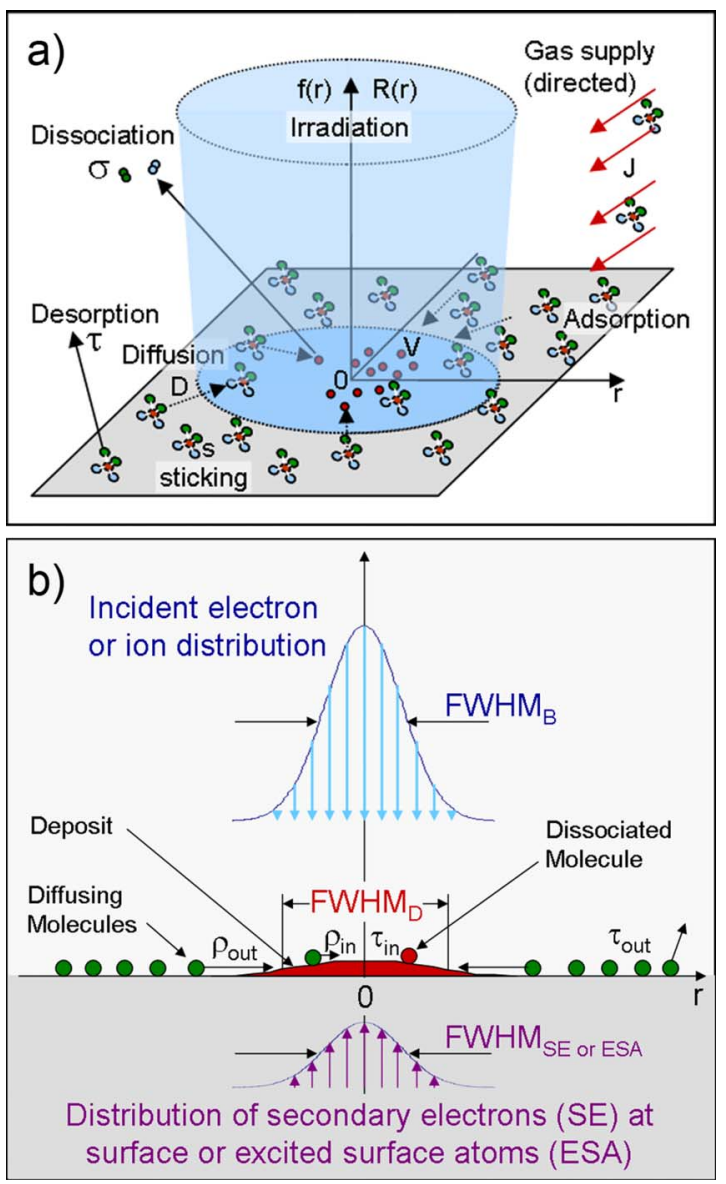

FIG. 24. Reference system and processes involved in FEB induced deposition. Inside the irradiated area precursor molecules are depleted by dissociation. Replenishment occurs by gas phase transport and by diffusion. The symbols $J, s, \tau, D$, and $\sigma$ denote the molecule flux, the sticking probability, the residence time, diffusion coefficient, and the dissociation cross section. $V$ is the volume of the decomposed molecule. (b) Schematics of incident beam size, size of generated secondary distributions, and deposit size in terms of the full width at half maximum (FWHM). Additionally, the effective residence times $\tau$ and the diffusion paths $\rho$ inside and outside the irradiated area are indicated.

One should keep in mind that cross sections calculated from measured deposition or etch rates are integral values over the entire energy spectrum and all fragmentation channels responsible for fixation or etching. Consequently, they are reaction parameters adequately reflecting the gasassisted FEB or FIB process with a given material system under given reaction conditions. Depending on the domination of the one or other fixation mechanisms, the energy dependence of such obtained cross section might condense into one of the types described in Secs. II B or II C. Furthermore, the volatile reaction products will have their specific surface residence times, which might be different from the residence time of the intact molecule.

The continuum model formalism can be applied to both FIB and FEB gas-assisted processing. The energy integral in Eq. (2.1a) and (2.1b) is approximated by the product $\sigma f(r)$ so that the deposition or etch rate $R$ (in units of dimension per unit time) becomes 


$$
R(r)=V n(r) \int_{0}^{E_{P E}} \sigma(E) f(r, E) d E \cong V n(r) \sigma f(r)
$$

For ion beams the physical sputter term has to be included (see Sec. II A). The spatial distribution of the emitted energy spectrum [see Fig. 24(b)] due to secondary electrons at the surface or excited surface atoms is assumed to equal the incident beam distribution. This is the case for low-aspect ratio deposits or etch holes and when the incident beam size is large compared to the size of the emitted spectrum, as discussed in Sec. II A 3 and summarized in Table IV. Furthermore, precursor depletion and replenishment is calculated solving the adsorption rate given in Eq. (2.2) for $n(r)$ assuming a planar surface.

We will start reviewing the steady state solutions of Eq. (2.2) with and without diffusion, which results in the important definition of (a) scaling laws for resolution of lowaspect-ratio deposits (etch holes) in terms of fundamental dimensionless variables and (b) in the definition of three FEB and FIB gas-assisted process regimes.

Subsequently, the time dependent solution of Eq. (2.2) is presented and the use of such solutions for extraction of molecule and interaction parameters is discussed. Finally, we specify the conditions for the electron or ion flux limited regime and briefly introduce models involving several different molecule species on the surface. Of note is that the concepts developed for focused charged particle deposition and etching can also be applied to focused beams of photons, i.e., laser induced deposition and etching. ${ }^{189}$

\section{A. Steady state solutions}

Solving Eq. (2.2) for steady state $d n / d t=0$ and neglecting the diffusion term, we obtain $n(r)=s J \tau_{\text {eff }}(r)$ with the effective residence time of the molecules $\tau_{\text {eff }}(r)=\left(s J / n_{0}+1 / \tau\right.$ $+\sigma f(r))^{-1}$. The deposition or etch rate becomes

$$
R(r)=s J \tau_{\mathrm{eff}}(r) \operatorname{V\sigma f} f(r)
$$

and represents the deposit or etch shape at a given time (see Fig. 25). For any peak function $f(r)$ with a peak value $f_{0}$ $=f(r=0)$, an effective residence time in the center of the electron or ion beam is defined: $\tau_{\text {in }}=\tau_{\text {eff }}(r=0)=1 /\left(s J / n_{0}\right.$ $\left.+1 / \tau+\sigma f_{0}\right)$. The effective residence time far away from the electron beam center becomes $\tau_{\text {out }}=\tau_{\text {eff }}(r \rightarrow \infty)=1 /\left(s J / n_{0}\right.$ $+1 / \tau)$. Hence, the molecule density outside the irradiated area is $n_{\text {out }}=s J \tau_{\text {out }}$ and at the beam center $n_{\text {in }}=s J \tau_{\text {in }}$. Inserting $\tau_{\text {in }}$ into Eq. (3.2) gives $R(r=0)=V \sigma f_{0} \cdot s J /\left(s J / n_{0}+1 / \tau+\sigma f_{0}\right)$, which is also the solution for a homogeneous incident beam already derived in the 1950s (Refs. 4 and 8) (sometimes differing by the term $s J / n_{0}$ according to the adsorption model used).

We now define three fundamental dimensionless parameters characterizing the gas-assisted FEB or FIB process. The residence time ratio,

$$
\tilde{\tau}=\tau_{\text {out }} / \tau_{\text {in }}=1+\sigma f_{0} /\left(1 / \tau+s J / n_{0}\right),
$$

represents a measure for depletion of precursor molecules due to dissociation at the center of the beam. The value $\tilde{\tau}$

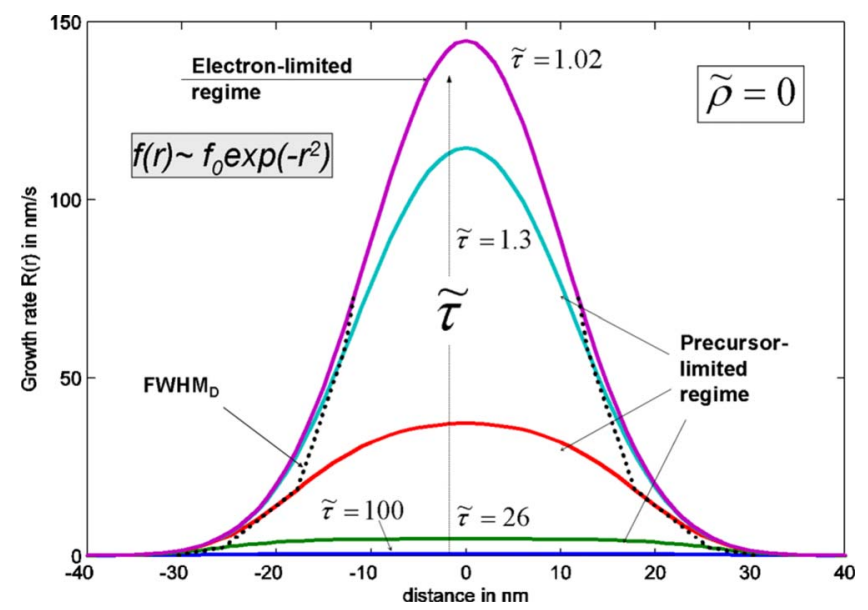

FIG. 25. Calculated deposit shapes according to Eq. (3.2) for differing molecule depletion $\widetilde{\tau}$ (no diffusion, $\widetilde{\rho}=0$ ). The incident Gaussian beam has a FWHM of $24 \mathrm{~nm}$. The FWHM of the deposit $\left(\mathrm{FWHM}_{D}\right)$ is increasing with increasing depletion.

$=1$ holds for a zero intensity beam or zero interaction with the molecule. Furthermore, we define the dimensionless deposit or etch resolution as the FWHM ratio of deposit (etch hole) and incident beam,

$$
\widetilde{\varphi}=\mathrm{FWHM}_{D} / \mathrm{FWHM}_{B} \text {. }
$$

$\mathrm{FWHM}_{D}$ and $\mathrm{FWHM}_{B}$ are given by the FWHM of $R(r)$ $=s J \tau_{\text {eff }}(r) \operatorname{Vof} f(r)$ and $f(r)$, respectively [see Fig. 24(b)]. The amount of replenishment by surface diffusion is given by the molecule diffusion path inside the irradiated area $\rho_{\text {in }}$ $=\left(D \tau_{\text {in }}\right)^{1 / 2}$ with respect to the beam size,

$$
\tilde{\rho}=2 \rho_{\text {in }} / \mathrm{FWHM}_{B} .
$$

For completeness we mention that the diffusion path outside the irradiated area is $\rho_{\text {out }}=\left(D \tau_{\text {out }}\right)^{1 / 2}$. By means of the dimensionless parameters defined in Eq. (3.3a), (3.3b), and (3.3c), the scaling law of resolution as a function of irradiative depletion $\widetilde{\varphi}(\widetilde{\tau})$ is conveniently expressed as ${ }^{218}$

$$
\widetilde{\varphi}=\left\{\log _{2}(1+\widetilde{\tau})\right\}^{m},
$$

with $m=\frac{1}{2}$ for a Gaussian distribution $f(r)=f_{0} \exp \left(-r^{2} / 2 a^{2}\right)$.

Taking the diffusion term in Eq. (2.2) into account and solving for $d n / d t=0$ and rotational symmetry, an analytical solution can be obtained for a cylindrical flat top distribution $f(r)=f_{0}$ for $|r| \leqslant \mathrm{FWHM}_{B} / 2$. The molecule density becomes (in our notation) $)^{219}$

$$
n(r)=n_{\text {out }}\left\{\widetilde{\tau}^{-1}+C_{2} I_{0}\left(r / \rho_{\text {in }}\right)\right\},
$$

where $I_{0}$ is the modified Bessel function. The diffusion contribution $C_{2}$ is given in Appendix E. A one dimensional solution for large rectangular scan windows is given by Ref. 220. The solution of $n(r)$ in Eq. (3.5) is represented graphically in Fig. 26.

Three important FEB and FIB process regimes can be distinguished: diffusion enhanced, precursor limited, and 

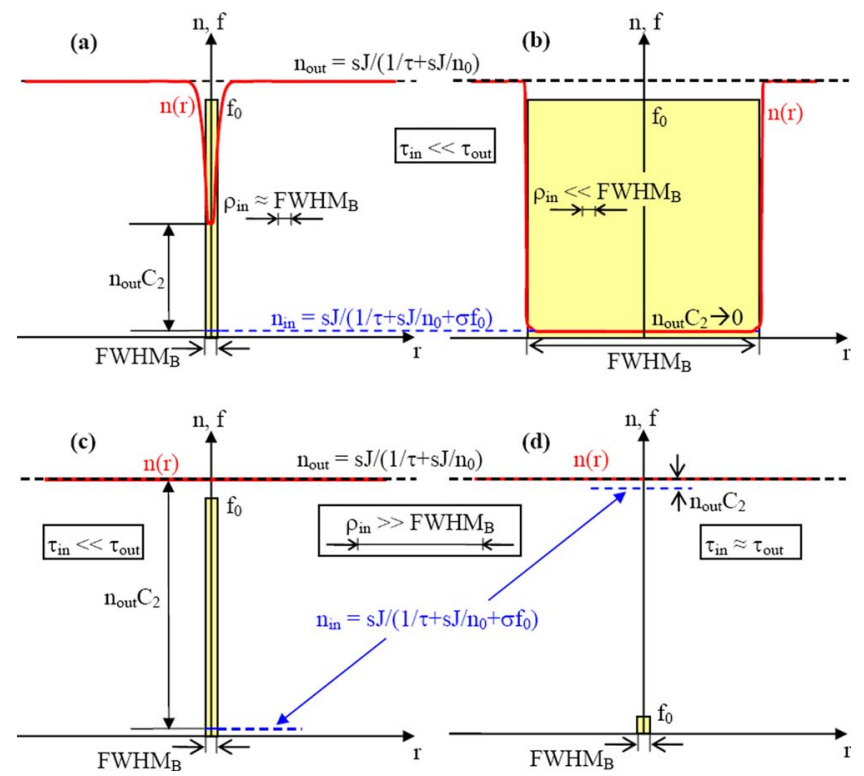

Fig. 26. Molecule density $n(r)$ in different FEB and FIB process regimes (red line). The beam size $\left(\mathrm{FWHM}_{B}\right)$ and beam flux intensity $\left(f_{0}\right)$ is highlighted in yellow (for simplicity the beam flux distribution is flat top and the $\mathrm{FWHM}_{B}$ thus corresponds to the cylinder diameter). The density $n_{\text {out }}$ is established outside the irradiated area and $n_{\text {in }}$ (blue dashed line) represents the density inside the irradiated area without diffusion. The degree of depletion depends on the ratio of the effective residence times $\tau_{\text {in }}$ and $\tau_{\text {out }}$ defined in the context of Eq. (3.2). The replenishment by diffusion in the center is given by $n_{\text {out }} C_{2}$. It increases with increasing diffusion path inside the irradiated area $\rho_{\text {in }}$ and decreasing size of the irradiated area. (a) Diffusionenhanced regime. The diffusive replenishment recovers part of the irradiative depletion. (b) Precursor-limited regime. The diffusion contribution is negligible since the diffusion path is small compared to the size of the irradiated area (beam diameter). (c) Electron- or ion-limited regime. The diffusive replenishment fully compensates irradiative depletion due to the large ratio of diffusion path vs size of irradiated area. (d) Electron- or ionlimited regime at low irradiative depletion.

electron or ion limited. Respectively, the terms mixed regime, precursor mass limited, and reaction limited can be found in the literature.

(1) The diffusion-enhanced regime is characterized by a dominance of molecule replenishment by surface diffusion with respect to precursor adsorption from the gas phase supply.

(2) The precursor-limited regime is characterized by negligible amount of surface diffusion and the process rate is determined by adsorption from the gas phase. The electron or ion limited regime is characterized by a fast replenishment rate compared to the molecule dissociation rate. This can be due to low dissociation efficiency (small dissociation cross section), a low incident ion or electron flux, a high molecule adsorption rate from the gas phase, or high molecule supply by diffusion on the surface.

Sometimes it is practical to pool the diffusion-enhanced regime and the precursor-limited regime in a more general molecule-limited regime. However, the diffusion-enhanced regime deserves a specific consideration since it is beam size dependent, i.e., it becomes operative when molecules reach the depleted center of irradiation by surface diffusion, $\tilde{\rho} \approx 1$. With increasing diffusive replenishment the deposition or etch rate increases from

$$
R=n_{\text {in }} V \sigma f_{0} \text { for } \tilde{\rho} \ll 1
$$

to

$$
R=n_{\text {out }} V \sigma f_{0} \text { for } \tilde{\rho} \gg 1 .
$$

Within these limits the scaling law for the FEB or FIB deposition or etch rate as a function of the FWHM of the beam was derived to ${ }^{219}$

$$
R(\mathrm{FWHM}) \propto 1 / \mathrm{FWHM}_{B}^{2},
$$

for a flat top beam distribution, but also approximately holding for a Gaussian incident beam profile. The maximum diffusion enhancement in deposition rate becomes $R(\tilde{\rho}$ $\rightarrow \infty) / R(\widetilde{\rho}=0)=\widetilde{\tau}$ at $r=0$. Experimentally, a variation of the contamination rate over four orders of magnitude was shown. ${ }^{219}$

Solving Eqs. (2.2) and (3.1) for a Gaussian distribution reveals all shapes observed in experiments: from flat top shapes (precursor-limited regime) to indented and round shapes (diffusion-enhanced regime) to Gaussian peaked shapes (electron-ion-limited regime), see Fig. 27(a). The indented shapes are due to diffusing precursor molecules fixed at the periphery of the impinging beam. ${ }^{221}$ Both deposition rate and resolution increase with increasing diffusive replenishment. For $\tilde{\rho} \gg 1, R(r)=s J \tau_{\text {out }} V \sigma f(r)$ since any depletion is entirely compensated by diffusion. In other words, the electron-limited regime is established and the deposit shape corresponds to the electron or ion beam distribution $f(r)$. A universal graph relating the dimensionless resolution to irradiative depletion and diffusive replenishment, $\widetilde{\varphi}(\widetilde{\tau}, \widetilde{\rho})$, is shown in Fig. 27(b) holding independently of how diffusive replenishment is experimentally achieved: either via the beam size $\mathrm{FWHM}_{B}$ (using the focus of the beam) or via the diffusion path $\rho_{\text {in }}$ (changing precursor diffusion).

The scaling law of resolution versus diffusive replenishment $\widetilde{\varphi}(\widetilde{\rho})$ is ${ }^{218}$

$$
\widetilde{\varphi} \cong\left\{\log _{2}\left(2+1 / \widetilde{\rho}^{2}\right)\right\}^{m},
$$

with $m=\frac{1}{2}$ for a Gaussian distribution $f(r)=f_{0} \exp \left(-r^{2} / 2 a^{2}\right)$. For high-aspect-ratio structures the diffusion becomes one dimensional along the length dimension. Aristov ${ }^{222}$ and Kislov $^{223}$ presented an analytical solution of the molecule density for a freestanding rod being deposited by a horizontally moving electron beam. The expression is similar to Eq. (3.5) but the diffusion contribution term contains now the dependence on the rod length, being inversely proportional to it. Formally, this solution should be applicable also to high-aspect cylinders deposited or etched coaxially to the beam provided that beam heating effects can be neglected. Summarizing, the continuum model scaling laws predict that the highest the resolution and deposition or etch rate, the better the precursor is replenished in the irradiated area or, in other words, the better molecule depletion is avoided. The 

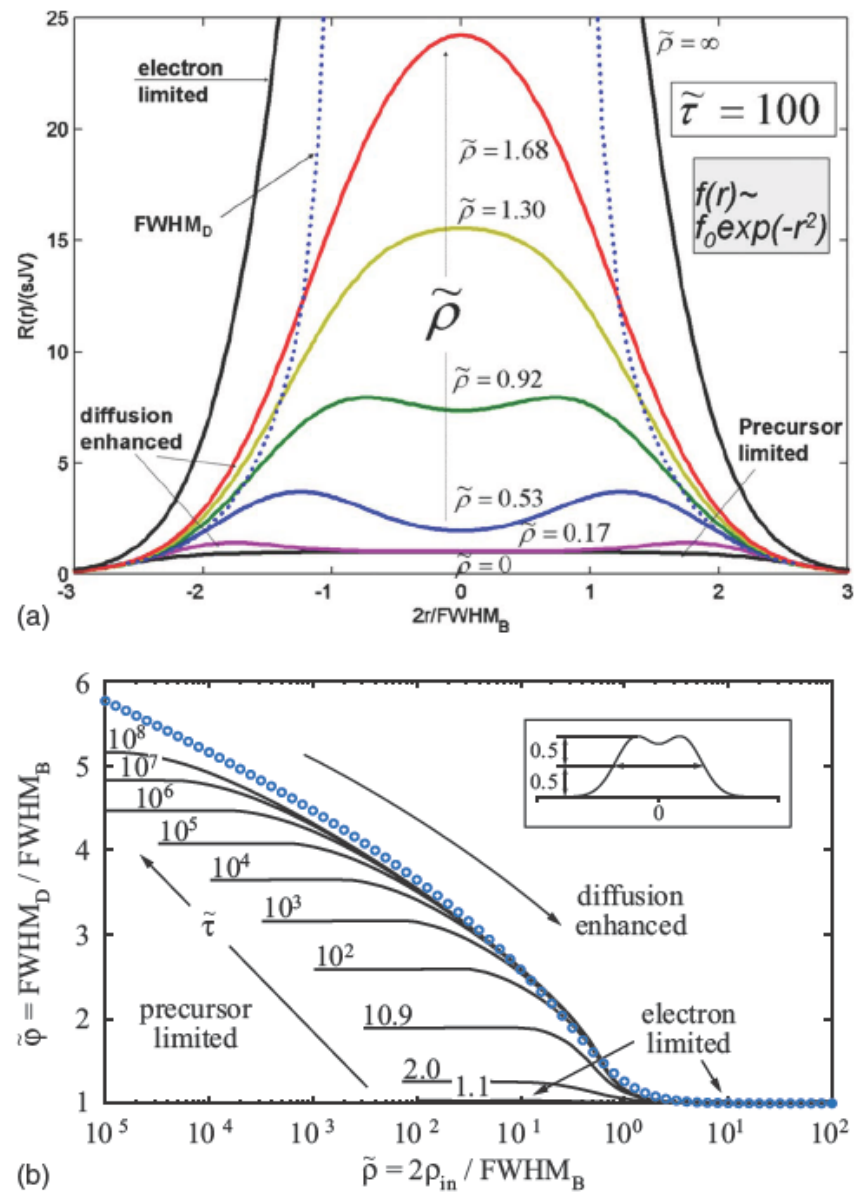

FIG. 27. (a) Normalized steady state deposition rate at indicated depletion representing the deposit shape. Note the shape transition from flat top, $\tilde{\boldsymbol{\rho}}$ $=0$, indented, $\tilde{\rho}=0.17$, round, $\tilde{\rho}=1.3$, to Gaussian, $\tilde{\rho}=\infty$, and the related decrease of the deposit FWHM (FWHM $\left.{ }_{D}\right)$ with increasing diffusive replenishment $\tilde{\rho}$. (b) Normalized deposit size vs normalized diffusion path for varying depletion (indicated). At $\tilde{\boldsymbol{\rho}}=2$ the diffusion path equals the beam size. Circles represent the scaling law in Eq. (3.8). The inset shows the $\mathrm{FWHM}_{D}$ definition of indented deposits (from Ref. 218).

smaller the beam size, the better molecule replenishment can be achieved by surface diffusion.

\section{B. Parameter determination from steady state exposures}

Generally, Eqs. (2.1a), (2.1b), and (2.2) contain six unknowns for FEB deposition, namely, $s, J, V, \sigma, \tau$, and $D$. Surely, $V$ could be considered as known in etch experiments. Sometimes $n_{0}$ is also unknown when the molecule physisorbs only on specific adsorption sites. To get a fully determined system for parameter extraction, six to seven independent experiments would be needed. Exposures using defocused beams with uniform irradiation $f$ were frequently performed since the diffusion contribution is only peripherical and does not affect the central irradiation zone [see Fig. 26(b)]. The deposition rate then becomes
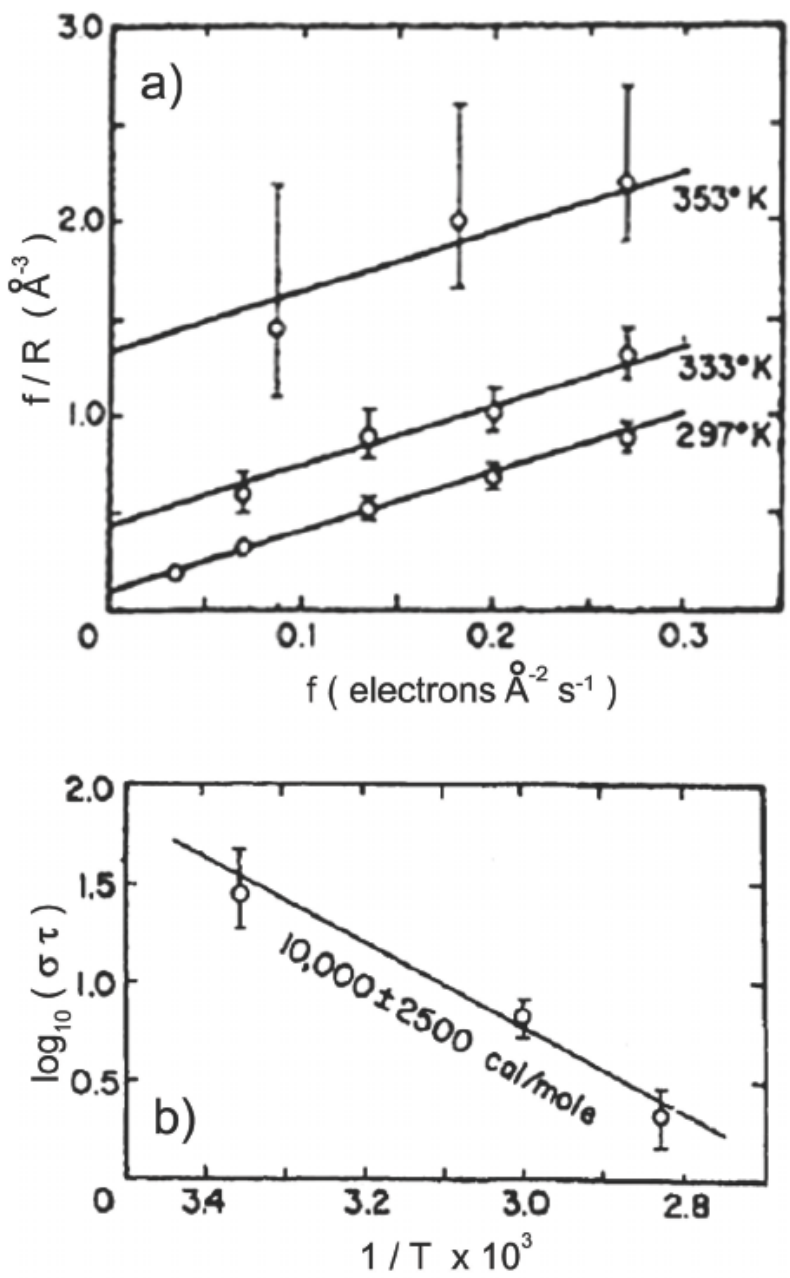

Fig. 28. (a) $f / R$ vs $f$ plot for electron beam induced deposition using methylphenylpolysiloxane (pump oil DC 704) and a $225 \mathrm{eV}$ beam (from Ref. 8). (b) Product of the electron impact cross section with the residence time of the oil molecule on the deposit vs reciprocal absolute temperature. The slope gives the desorption enthalpy of $0.43 \mathrm{eV}$ (from Ref. 8).

$$
R=n V \sigma f=\frac{s J}{s J / n_{0}+1 / \tau+\sigma f} V \sigma f .
$$

Varying the electron or ion flux $f$ allows us to test the fundamental assumption of a second order kinetics relating $R$ proportionally to the product $n f$. Generally this proportionality is valid except for a square root relation that was found for the polymerization of butadiene molecules by electron irradiation. ${ }^{224}$ Expressing Eq. (3.9) as ${ }^{8,187}$

$$
\frac{f}{R}=\frac{s J / n_{0}+1 / \tau}{V s J \sigma}+\frac{1}{V s J} f
$$

allows determining the product $s J V$ in a Cartesian plot from the slope of $f / R$ vs $f$ [see Fig. 28(a)]. The precursor flux $J$ and the specific volume of the fixed decomposed molecule must be known to determine the sticking probability $s$. The intersection point at $f=0$ is $\left(V n_{0} \sigma\right)^{-1}$ for $s J / n_{0} \gg 1 / \tau$. This condition must be validated when publishing cross sections $\sigma$ for FEB or deposition yields $Y_{D}=n_{0} \sigma$ for FIB and can be achieved by cooling the substrate. ${ }^{187}$ FEB cross sections 


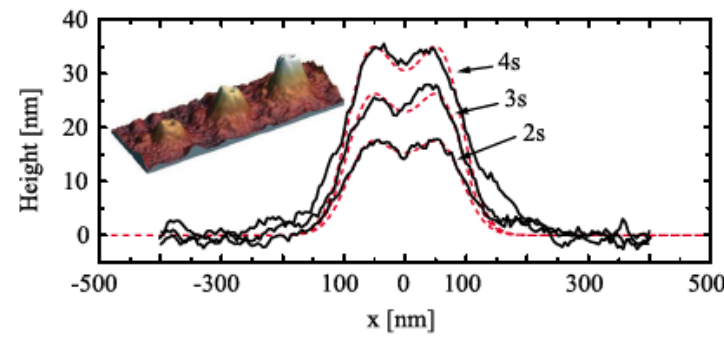

FIG. 29. Shape fitting with continuum model. AFM image and line scans of FEB deposits from $\mathrm{Cu}(\mathrm{hfa})_{2}$ precursor. Exposure times are indicated. Fits (dashed lines) were obtained with $\sigma=0.6 \mathrm{~nm}^{2}$ (=molecule size), $\tau=10^{-3} \mathrm{~s}$ (typical value), and $D=4 \times 10^{-7} \mathrm{~cm}^{2} \mathrm{~s}^{-1}$. Other deposition parameters: Gaussian beam with FWHM $=110 \mathrm{~nm}(5 \mathrm{keV})$ and $f_{0}=9 \times 10^{4} \mathrm{~nm}^{-2} \mathrm{~s}^{-1}$. Precursor supply with $s J / n_{0}=10 \mathrm{ML} / \mathrm{s}$ (from Ref. 218).

were measured, for instance, for $\mathrm{Ru}_{3}(\mathrm{CO})_{12} \quad(\sigma=2$ $\left.\times 10^{-3} \mathrm{~nm}^{2}, 50 \mathrm{keV}\right)\left(\right.$ Ref. 187) and benzol $\left(\sigma=0.35 \mathrm{~nm}^{2}\right.$, $200 \mathrm{eV}) .{ }^{110}$ For FIB examples of cross sections include $\mathrm{W}(\mathrm{CO})_{6} \quad\left(\sigma=10 \mathrm{~nm}^{2}, 42 \mathrm{keV} \mathrm{Ga}^{+}\right) \quad$ (Ref. 88) and $\mathrm{Me}_{2}-\mathrm{Au}(\mathrm{hfa})\left(\sigma=52 \mathrm{~nm}^{2}, 40 \mathrm{keV} \mathrm{Ga}\right)^{+}{ }^{58}$ For $s J / n_{0} \ll 1 / \tau$, the intersection point becomes $(s J V \sigma \tau)^{-1}$ and only the product $\sigma \tau$ can be determined correctly. ${ }^{8}$ Increasing the temperature will leave the cross section unaltered but decrease the residence time according to $\tau=\tau_{0} \exp \left(E_{\mathrm{des}} / k T\right)$, so that desorption energies $E_{\text {des }}$ of molecules (or molecule fragments) on the irradiated deposit can be determined from $\sigma \tau$ [see fig. 28(b)], representing the real experimental conditions. As a rule of thumb, roughly one-fifth of the desorption energy can be considered as the activation energy for surface diffusion (see Sec. II E 1). The deposited molecule volume is determined by density and composition measurements (see Sec. VI A 3).

When focused beams are employed for deposition and etching, diffusion should be taken into account. If the distribution $f(r)$ and the local precursor flux are known, already a simple dot deposition experiment allows making lower limit estimations of the cross section and diffusion coefficient from the FWHM ratio of the beam and the deposit. ${ }^{218}$ According to Fig. 27(b) the same FWHM ratio of deposit to beam can be achieved for larger depletion and larger diffusive replenishment or, in other words, larger $\sigma$ and $D$. Additional experiments are needed for their unique determination.
The shapes of deposits as shown in Fig. 29 give further details, like indents, to match which further limits the fit range of the parameters.

The shape fits shown in Fig. 29 and their parameters are summarized in Table VIII together with other experiments.

From Table VIII follows that the measured adsorption energy is in the range of reversible physisorption (compared to energies of chemisorption in the $1 \mathrm{eV}$ range). It was noted that extraordinarily high attempt periods $\tau_{0}$ result from the relation $\tau=\tau_{0} \exp \left(E_{\text {des }} / k T\right)$ compared to $\tau_{0} \approx 1 \times 10^{-12} \mathrm{~s}$ obtained from the classical transition state theory. ${ }^{189}$ They might be due to the large degrees of internal vibrational states for large molecules or it might be due to a possible ionization of the molecule that prevents it from normal desorption. Instead it will stay in contact with the (un)charged surface. ${ }^{226} \mathrm{An}$ increase of the molecule desorption (adsorption) energy with decreasing electron beam current and decreasing beam energy was found in $\mathrm{WF}_{6}$ deposition ${ }^{227}$ [whereas Fig. 28(a) shows no dependence for methylphenylpolysiloxane]. It was suggested that secondary electron stimulated desorption of fluorine species dominated at high beam currents and low beam energies over natural thermal desorption. Generally, published values of diffusion coefficients, residence times, and cross sections (yields) determined from FEB and FIB deposition or etch experiments are very limited and show a large scatter (see also Sec. III D). For these reasons it is difficult to draw firm conclusions about possible irradiation enhancement effects. Further well defined experiments (excluding process regime dependencies) are definitely needed to better quantify the diffusion, adsorption, and dissociation behavior of molecules under irradiation.

\section{Time dependent solutions for pulsed irradiation}

In the case of negligible diffusion (broad beams or large scan fields), the adsorption rate equation (2.2) can be solved analytically and gives the general time dependent solution

$$
n(t)=C e^{-k t}+B .
$$

Denoting "beam on" (depletion) and "beam off" (replenishment) with subscripts $d$ and $r$, respectively, the constants $k$, $B$, and $C$ can be obtained from $n_{d}(t=0)=n_{\text {out }}, n_{d}(t=\infty)=n_{\text {in }}$,

TABLE VIII. Parameters determined from focused electron, ion, and photon induced deposition and etching experiments using the continuum model. The diffusion coefficient and the residence time are given at room temperature.

\begin{tabular}{lcccccc}
\hline \hline $\begin{array}{l}\text { Beam; } \\
\text { precursor }\end{array}$ & $\begin{array}{c}\text { Incident } \\
\text { energy }\end{array}$ & $\begin{array}{c}\text { Cross } \\
\text { section } \\
\left(\mathrm{nm}^{2}\right)\end{array}$ & $\begin{array}{c}\text { Residence } \\
\text { time }\end{array}$ & $\begin{array}{c}\text { Diffusion } \\
\text { coefficient } \\
\left(\mathrm{cm}^{2} / \mathrm{s}\right)\end{array}$ & $\begin{array}{c}\text { Desorption } \\
\text { energy } \\
(\mathrm{eV})\end{array}$ & Ref. \\
\hline $\begin{array}{l}\mathrm{FEB} ; \mathrm{Cu}(\mathrm{hfa})_{2} \\
\mathrm{Ga}-\mathrm{FIB} ;\end{array}$ & $5 \mathrm{keV}$ & $0.1-0.6$ & $\ldots$ & $(0.3-4) \times 10^{-7}$ & $\ldots$ & 218 \\
$\begin{array}{l}\mathrm{Si} \text { etch with } \mathrm{Cl}_{2} \\
\text { FEB; } \\
\text { contamination }\end{array}$ & $10 \mathrm{keV}$ & 6.5 & $80 \mathrm{~ms}$ & $8 \times 10^{-6}$ & $0.2-0.3$ & 225 \\
$\begin{array}{l}\text { Ar-laser; } \\
\text { Tetraethyl lead }\end{array}$ & $100 \mathrm{keV}$ & $0.007-0.02$ & $\ldots$ & $6.4 \times 10^{-10}$ & $\ldots$ & 223 \\
\hline \hline
\end{tabular}




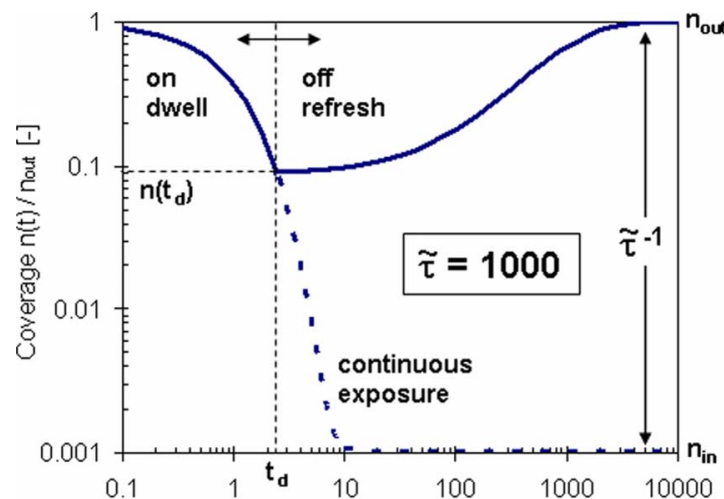

(a)

Time in $k_{d} \cdot t[-]$

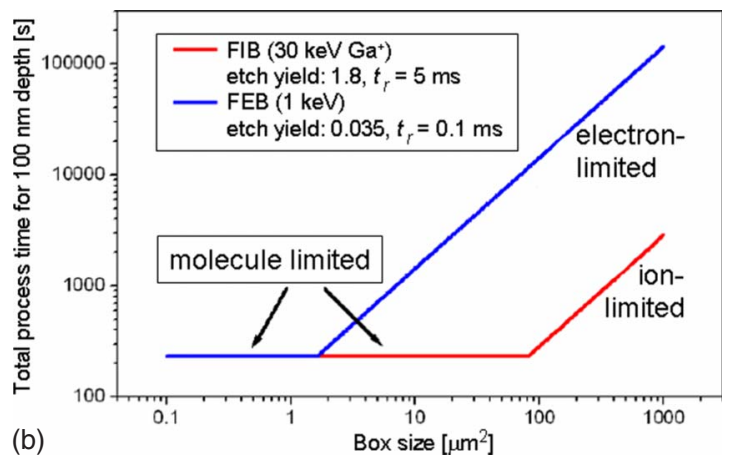

FIG. 30. (a) Normalized molecule density $n(t) / n_{\text {out }}$ for one on/off cycle and a continuous exposure. Typical dwell times are in the microsecond range whereas the refresh time range is around several milliseconds. Here we have chosen $t_{d}=1 \times 10^{-4} \cdot t_{r}$ and an irradiative depletion $\tilde{\tau}=1000$. Note that depletion can be effectively reduced due to small dwell times and long replenishment (refresh) cycles. (b) Comparison of the total process time for gasassisted FEB and FIB etching of a $100 \mathrm{~nm}$ deep box into $\mathrm{SiO}_{2}$. For large boxes the total process time becomes electron and ion limited. At small box sizes the process (and the throughput) becomes molecule limited (courtesy of K. Edinger, NaWoTec GmbH).

$n_{r}(t=0)=n_{\text {in }}$, and $n_{r}(t=\infty)=n_{\text {out }} \cdot n_{\text {in }}$ and $n_{\text {out }}$ were defined in Sec. III A giving,

$$
k= \begin{cases}k_{d}=1 / \tau_{\text {in }}=s J / n_{0}+1 / \tau+\sigma f & \text { on } \\ k_{r}=1 / \tau_{\text {out }}=s J / n_{0}+1 / \tau & \text { off }\end{cases}
$$

and

$$
B= \begin{cases}B_{d}=s J / k_{d}\left(=n_{\text {in }}\right) & \text { on } \\ B_{r}=s J / k_{r}\left(=n_{\text {out }}\right) & \text { off. }\end{cases}
$$

The constants $C$ become $C_{d}=-C_{r}=B_{r}-B_{d}$. The solutions of Eq. (3.11a) for a pulsed beam are found by imposing the corresponding periodic steady state conditions $n_{d}(0)=n_{r}\left(t_{r}\right)$ and $n_{r}(0)=n_{d}\left(t_{d}\right), t_{d}$ being the dwell time (depletion) and $t_{r}$ the refresh time (replenishment). This yields for $C$,

$$
C= \begin{cases}C_{d}=\left(B_{r}-B_{d}\right)\left(e^{-k_{r} t_{r}}-1\right)\left(e^{-k_{r} t_{r}} e^{-k_{d} t_{d}}-1\right)^{-1} & \text { on } \\ C_{r}=\left(B_{d}-B_{r}\right)\left(e^{-k_{d} t_{d}}-1\right)\left(e^{-k_{r} t_{r}} e^{-k_{d} t_{d}}-1\right)^{-1} & \text { off. }\end{cases}
$$

Figure 30 shows the normalized adsorbate coverage for one on/off cycle.
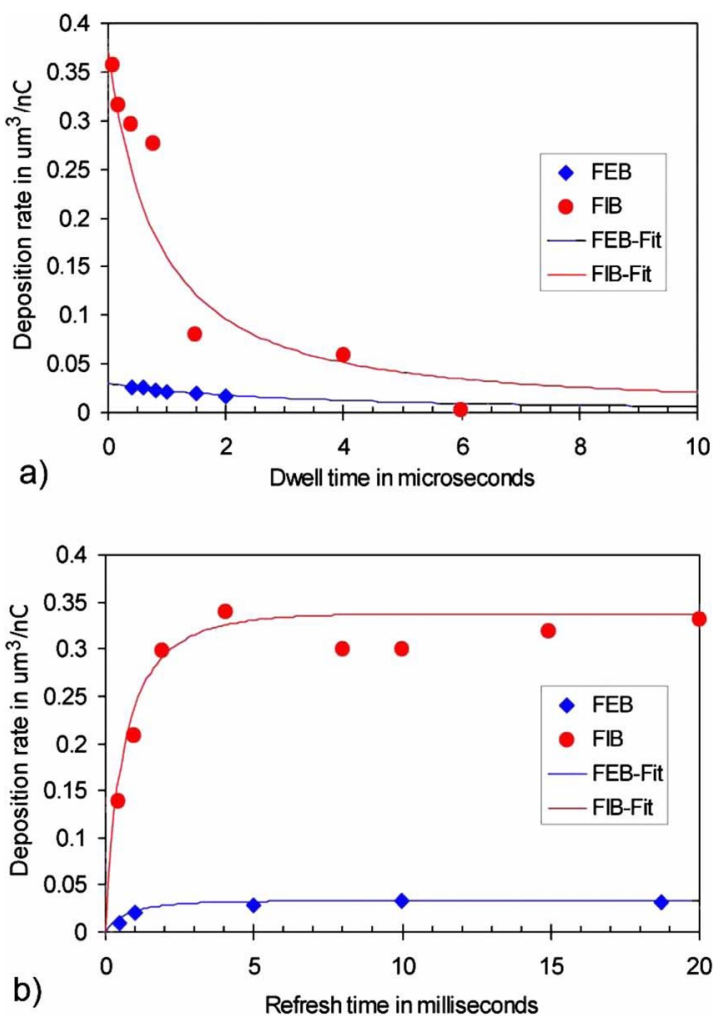

FIG. 31. Comparison of FEB (10 keV) and FIB (30 keV $\left.\mathrm{Ga}^{+}\right)$deposition rates with $\mathrm{Si}\left(\mathrm{OCH}_{3}\right)_{4}$ precursor (TMOS). Solid lines represent the analytical solution of Eq. (3.13) fitted to the experimental data. (a) The dwell time variation was performed at $t_{r}=10 \mathrm{~ms}$. (b) The refresh time variation was performed at $t_{d}=200 \mathrm{~ns}$. Experimental data and FIB fits were reproduced from Ref. 231.

Often $n_{\text {in }} \ll n_{\text {out }}$ and $n(t) \approx n_{\text {out }} \exp \left(-k_{d} t\right)$ for $t \leqslant k_{d}^{-1} \ln (\widetilde{\tau})$, which is about $t<7 k_{d}^{-1}$ for $\tilde{\tau}=10^{3}$ [see Fig. 30(a)]. The scaling law relating the dwell time to deposit or etch resolution is found making use of Eq. (3.4) and the exponential increase of depletion with dwell time, ${ }^{218}$

$$
\widetilde{\varphi}=\left\{\log _{2}\left(1+\exp \left(k_{d} t_{d}\right)\right\}^{m},\right.
$$

with $m=\frac{1}{2}$ for a Gaussian distribution and $t \leqslant k_{d}^{-1} \ln (\widetilde{\tau})$. Explicitly, the condition $t_{d} \leqslant 0.27 k_{d}^{-1}$ is found when the lateral deposit or etch size should not exceed $10 \%$ of the $\mathrm{FWHM}_{B}$ of a Gaussian incident beam.

The chemically driven deposition or etch rates during the exposure interval $t_{d}$ is given by

$$
\begin{aligned}
R= & \frac{V \sigma f}{t_{d}} \int_{0}^{t_{d}} n(t) d t \\
= & \operatorname{V\sigma f}\left\{\frac{\left(B_{r}-B_{d}\right)}{k_{d} t_{d}} \frac{\left[1-\exp \left(-k_{r} t_{r}\right)\right]\left[1-\exp \left(-k_{d} t_{d}\right)\right]}{1-\exp \left(-k_{r} t_{r}\right) \exp \left(-k_{d} t_{d}\right)}\right. \\
& \left.+B_{d}\right\},
\end{aligned}
$$

in units of unit dimension per unit time. Similar expressions with differing notations can be found in Refs. 59, 88, and 228-232. The solutions $R\left(t_{d}\right)$ and $R\left(t_{r}\right)$ are shown graphically in Fig. 31. Here $R$ is given in units of volume per 


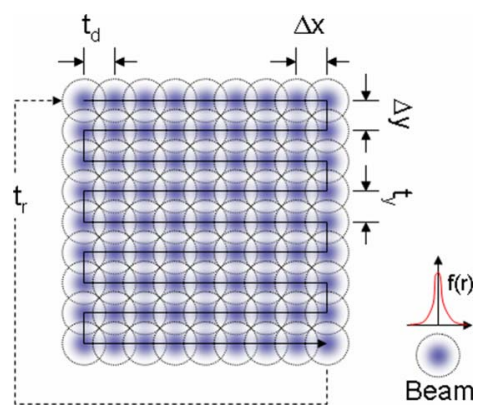

FIG. 32. Digital serpentine raster scan scheme with overlap. The (Gaussian) beam dwells for a time $t_{d}$ at $(x, y)$ and is then moved by $\Delta x$ and $\Delta y$ to the next exposure spot. The scan is repeated after a refresh time $t_{r}$.

incident charge $\left(\mu \mathrm{m}^{3} / \mathrm{nC}\right)$, which is obtained by dividing $R$ in Eq. (3.13) by $e_{0} f$. Basically, decreasing the pixel dwell time avoids molecule depletion to proceed down to its steady state value [see Fig. 30(a)]. The deposition or etch rate increases and saturates for dwell times smaller than the effective residence time inside the irradiated area $t_{d} \ll \tau_{\text {in }}$ [which is not visible on the linear scale in Fig. 31(a)]. Increasing the refresh time results in molecule replenishment of all the irradiated pixels before the next irradiation cycle begins. The deposition and etch rate increase and finally saturate for refresh times larger than the effective residence time outside the irradiated area $t_{r} \gg \tau_{\text {out }}$. Obviously, for a dwell time $t_{d}$ $\ll \tau_{\text {in }}$ and a refresh time $t_{r}>\tau_{\text {out }}$, the maximum rate $R$ $=n_{\text {out }} V \sigma f$ is achieved due to negligible depletion. This is why the higher efficiency in deposition with ions compared to electrons levels off in the total process time for small scan windows [see Fig. 30(b)]. Within the electron- or ion-limited regime, there is a direct proportionality between total process time $t$ and box size $A$ for a given thickness $z$ to be etched or deposited. This follows from $t=z / R=z /(V \sigma n f)$ $=z A e_{0} /\left(V \sigma n I_{P}\right)$. The molecule density $n$ can be regarded as constant in the electron- or ion-limited regime.

\section{Parameter determination from raster scan exposures}

Often exposure of boxes is achieved via a serpentine scan shown in Fig. 32. The beam is moved in increments along $\Delta x$ and $\Delta y$ on the surface exposing each increment with a dwell time $t_{d}$. After finishing one raster scan, the beam repeats the pattern after a refresh time $t_{r}$, sometimes also called as loop time. Evidently, the minimum refresh time that can be realized in such a raster scan is given by the number of pixel exposures in the scan window times the dwell time for each pixel. Each pixel is exposed during a dwell time $t_{d}$ and, in case of overlap, also from adjacent pixels after multiples of $t_{d}$ and $t_{y}$ (see Fig. 32). For zero overlap and flat top distribution $f(r)$, the deposition or etch rate of this scan is given by Eq. (3.13). Experiments and theory are compared in Fig. 31. for FEB and FIB deposition. From the $R\left(t_{d}\right)$ and $R\left(t_{r}\right)$ curves the exponential exponents $k_{r}$ and $k_{d}$ can be fitted, hence the effective residence times $\tau_{\text {in }}$ and $\tau_{\text {out }}$ [see Eq. (3.11b)]. If $\sigma f \gg s J / n_{0}+1 / \tau$, the cross section can be determined $\sigma$ $\approx 1 /\left(\tau_{\text {in }} f\right)$, and if $s J / n_{0} \ll 1 / \tau$, the residence time can be estimated, $\tau \approx \tau_{\text {out. }}$. However, independent measurements must be performed to prove these relations. The bulk of published measurements for FEB and FIB shows that refresh times in the order of milliseconds are needed to achieve full replenishment of the exposed regions, thus implying that the residence time of molecules on the (irradiated) deposit is also in the millisecond range or even larger or that the incident gas flux $s J / \mathrm{n}_{0}$ was roughly $10^{3}$. Fitting the experimental data from Ref. 231 in Fig. 31 with Eq. (3.13) shows that the FIB deposition cross section is $\sigma=20 \mathrm{~nm}^{2}$ (Ref. 231) for TMOS and the FEB cross section is $\sigma=2 \mathrm{~nm}^{2}$ assuming as deposited density pure $\mathrm{SiO}_{2}$. The FEB cross section is comparatively high probably due to surface charge effects or polymerization. The energy dependence of the FEB deposition cross section for $\mathrm{W}(\mathrm{CO})_{6}$ shown in Fig. 11(b) was determined using the raster scan model and different incident electron energies. ${ }^{64}$

The numerical solution taking into account overlaps and Gaussian distributed beams ${ }^{59,89}$ could reproduce the shoulder effect seen in experiments: this effect is related to the serpentine raster scan, which delivers portions of the ion dose to a given pixel at different times. While in the fast horizontal scan direction the beam passes over neighboring pixels in a continuous way, successive exposures of the given pixel due to vertically adjacent pixels are separated by the time it takes the beam to complete one row. During this line refresh time, $t_{y}$ in Fig. 32, the pixel is replenished with precursor molecules. For $\mathrm{Cl}_{2}$-assisted etching of $\mathrm{Si}$ with $50 \mathrm{keV} \mathrm{Ga}^{+}$ions, two $\mathrm{Cl}_{2}$ molecules react with one $\mathrm{Si}$ atom, $m=2$, the chemical etch yield is $q=45$ atoms/ion, and the monolayer coverage is $n_{0}=6.5 \times 10^{14} \mathrm{~cm}^{-2} .{ }^{59}$ The conversion into the corresponding cross section is

$$
\sigma=m q / n_{0},
$$

giving $\sigma=14 \mathrm{~nm}^{2}$. The same paper gives for $\mathrm{I}_{2}$-assisted etching of $\mathrm{Si}$ with $25 \mathrm{keV} \mathrm{Ga} \mathrm{Ga}^{+}$ions the values $m=2, q=40$ atoms/ion, and $n_{0}=1.5 \times 10^{14} \mathrm{~cm}^{-2}$; hence $\sigma=53 \mathrm{~nm}^{2}$. The number $m$ of reacting precursor molecules is known from the chemical reactions $2 \mathrm{I}_{2}+\mathrm{Si} \rightarrow \mathrm{SiI}_{4}$ and $2 \mathrm{Cl}_{2}+\mathrm{Si} \rightarrow \mathrm{SiCl}_{4}$. The yield enhancement in gas-assisted FIB etching is obtained with respect to physical sputtering (without gas assistance). It represents a characteristic value for a materials system (substrate, precursor molecule, and the incident ions with a given energy). Gas-assisted enhancement factors in FIB sputter rates are comprehensively summarized in Sec. V D 2. Large differences in yield enhancements for the same material system are reported in different literature sources. This is primarily due to the fact that often replenishment of the precursor in the irradiated area was not fully achieved. In this case the enhancement factors rather characterize the depletion process than the materials system!

Summarizing, raster scan exposures can be used to determine parameters related to FEB and FIB gas-assisted processing. Surface diffusion is generally not considered in ras- 
TABLE IX. Calculated minimum diffusion coefficients $D$ and minimum exposure dwell times $t_{d}$ for the electron- or ion-limited regime. Typical ranges of incident peak flux $f_{0}$ and beam size $\mathrm{FWHM}_{B}$ are taken for a focused electron beam (5 keV, field emission filament) and an ion beam (30 kV, Ga). Ranges for the cross section $\sigma$ were chosen from literature results discussed in Sec. III B.

\begin{tabular}{lcccccc}
\hline \hline & & & & & & \\
& $f_{0}\left(1 / \mathrm{nm}^{2} \mathrm{~s}\right)$ & $\begin{array}{c}\mathrm{FWHM}_{B} \\
(\mathrm{~nm})\end{array}$ & $\sigma\left(\mathrm{nm}^{2}\right)$ & $\sigma f_{0}(1 / \mathrm{s})$ & $D\left(\mathrm{~cm}^{2} / \mathrm{s}\right)$ & $t_{d}(\mathrm{~s})$ \\
\hline FEB & $8 \times 10^{6}$ & 2.5 & $2 \times 10^{-4}$ & $1.6 \times 10^{3}$ & $1 \times 10^{-10}$ & $1.6 \times 10^{-4}$ \\
& $5 \times 10^{7}$ & 100 & 0.2 & $1 \times 10^{7}$ & $2.6 \times 10^{-8}$ & $1 \times 10^{-3}$ \\
FIB & $2 \times 10^{5}$ & 7 & 10 & $2 \times 10^{6}$ & $9.8 \times 10^{-7}$ & $1.3 \times 10^{-7}$ \\
& $5 \times 10^{6}$ & 100 & 50 & $2.5 \times 10^{8}$ & $2.5 \times 10^{-2}$ & $1.0 \times 10^{-9}$ \\
\hline \hline
\end{tabular}

ter scan models probably due to the enormous computation effort when solving the appropriate differential equation. Basically, the number of molecules inside the irradiated area is increased due to diffusion, which would be equivalent to an increased effective residence time. An analytic estimate of the increase in residence time due to diffusion gives

$$
\tau_{\text {diff }} \approx C_{2} \tau_{\text {out }},
$$

where $\mathrm{C}_{2}$ was introduced in Eq. (3.5) and Appendix E. The effective residence time inside the irradiated area including diffusion is thus $\tau_{\text {eff }}=\tau_{\text {in }}+\tau_{\text {diff }}$ and results in values of $k_{\text {eff }}$ $=1 / \tau_{\text {eff }}$ being lower than the $k$ values defined in Eqs. (3.11b). Thus determination of the cross section and the residence time from Eq. (3.11b) is prone to overestimation. Furthermore, the accuracy of the parameter determination depends on whether molecule replenishment is really achieved while decreasing the dwell time (and increasing the refresh time). Only then the conditions for the electron- or ion-limited regime are established and the yield determination becomes independent of the molecule replenishment.

\section{E. Conditions for the electron- or ion-limited regime}

It was shown that this regime, also referred to as reactionlimited regime, gives the highest resolution as well as the highest deposition or etch rate of the gas-assisted FEB/FIB process. This is why here we derive the conditions under which this regime is operative. We start with a compilation of maximum and minimum values of FIB and FEB cross sections found in literature together with typical beam sizes of field emitter SEMs and Ga-ion FIB columns shown in Table IX. This gives the range of molecule dissociation frequencies $\sigma f_{0}$ due to irradiation. If $\sigma f_{0}$ determines the effective residence time inside the irradiated area, then $\tau_{\text {in }}$ $=\left(k_{d}\right)^{-1}=\left(\sigma f_{0}\right)^{-1}$, and we can readily estimate ranges of diffusion coefficients and exposure times needed for establishing the electron-/ion-limited regime.

Compensation of depletion by surface diffusion requires a molecule diffusion path at least comparable to the beam size, $\tilde{\rho} \geqslant 2$, i.e., $\rho_{\text {in }} \geqslant \mathrm{FWHM}_{B} \quad\left[\right.$ see Fig. 27(b)]. With $\rho_{\text {in }}$ $=\left(D \tau_{\text {in }}\right)^{1 / 2} \approx\left(D / \sigma f_{0}\right)^{1 / 2}$ we obtain the condition for the diffusion coefficient $D \geqslant \sigma f_{0} \mathrm{FWHM}_{B}^{2}$ to satisfy the electron- or ion-limited regime.
When the condition $\rho_{\text {in }} \geqslant \mathrm{FWHM}_{B}$ cannot be met in a continuous exposure experiment, pulsed beams can be employed to stay in the electron-limited regime. The exposure (dwell) time ranges can be estimated from $\tau_{\text {in }}$ $\sim \tau_{\text {out }} \exp \left(-k_{d} t\right)=\tau_{\text {out }} \exp \left(-\sigma f_{0} t\right)$. Defining the criterion that the deposit or etch size should not much exceed the beam size, i.e., $\tilde{\varphi} \leqslant 1.1$, translates into a low depletion $\tilde{\tau}=\tau_{\text {out }} / \tau_{\text {in }}$ $\leqslant 1.3$ according to Eq. (3.4). Thus for the exposure time the condition $t \leqslant \ln (\widetilde{\tau}) / k^{-1}=0.26\left(\sigma f_{0}\right)^{-1}$ is obtained to satisfy the electron- or ion-limited regime. The low end of these time scales (see Table IX), has not yet been explored in focused particle beam induced processing.

\section{F. Models accounting for several species of adsorbates}

Different surface adsorbed molecule species arise when intermediate reaction products are formed or when two different precursor molecules are deliberately introduced into the vacuum chamber. In any of such cases the equilibrium surface density of each adsorbate species is obtained from a set of coupled differential adsorption rate equations. As a consequence, the surface density of a given adsorbate will depend on all adsorbate species present on the surface (in addition to the adsorbate's own set of parameters: dissociation cross section, residence time, diffusion coefficient, sticking probability, and gas phase supply). Of most interest are such "systems" under irradiation conditions for which the production of one species saturates due to depletion while the other species are still sufficiently replenished. This is equivalent to a regime transition from electron or ion limited to molecule limited of a given adsorbate species. Only under such conditions the composition among adsorbate species will change and, most importantly, also the deposit or process properties related to the physical or chemical action of this specific adsorbate. A few examples are reported in literature.

The formation of an intermediate product during $\mathrm{W}(\mathrm{CO})_{6}$ deposition with FEB was introduced by Ref. 233 in order to explain the irradiation dose dependency of resistance, thickness, and tungsten content of the deposit. At low irradiation doses (low number of electrons per area) the intermediate product was predominantly formed having a higher resistivity, larger molecule volume, and lower tungsten content com- 
pared to the final product. At higher irradiation doses the tungsten-rich final product formed with lower resistivity. In terms of process regimes the behavior can be explained as follows: at low doses (flux) the reaction is electron limited and proceeds to the intermediate product. At high doses the reaction is molecule limited, i.e., each molecule undergoes a larger number of electron collisions while at the surface, which will dissociate the intermediate product into the final product by releasing more $\mathrm{CO}$ ligands and leaving purer tungsten.

Another example is the competing mechanism of hydrocarbon contamination deposition and $\mathrm{H}_{2} \mathrm{O}$ etch molecules both adsorbed on the same surface. ${ }^{234}$ The switching between deposition (contamination pillar) and etching (substrate surface attacked) with increasing electron dose (flux) was observed. Again, the process regimes can conceptually explain this behavior. With increasing electron flux the contamination deposition rate becomes molecule limited (due to its low supply flux from the contaminated surface only) and saturates while the etching still proceeds in the electronlimited regime (sufficient $\mathrm{H}_{2} \mathrm{O}$ supply provided), i.e., the etch rate increases and takes over. A theoretical treatment of these competing processes in a stationary electron beam profile is given in Ref. 235. Since the deposition in the central irradiated region with highest electron flux is molecule limited, the competing etching process is dominantly active here. The final deposit is a ringlike structure with a wall resolution in the order of nanometers. These ringlike shapes have an analogy with the indented structures in Figs. 27 and 29. In both cases the central irradiated region is molecule limited. The analogy to ringlike structures obtained by stationary focused ion beam deposition is evident.

A model describing the composition of deposits obtained during $500 \mathrm{eV} \mathrm{Ar}^{+}$beam induced deposition from (hfa)CuVTMS is given by Ref. 236. The processes that could be quantified and modeled are (a) dissociative precursor adsorption, (b) ion induced $\mathrm{Cu}$ deposition and removal, (c) ion induced carbon deposition and removal, and (d) desorption of an intermediate carbon containing product. They prove that a faster deposition rate (higher ion flux) results in higher carbon impurity fraction due to the finite desorption rate of the carbon intermediate. This implies a dependency of electrical resistance on incident charged particle flux opposite to the one found by Hoyle discussed above. Obviously, such a dependency seems to be molecule specific. It can be seen that the concepts of process regimes, especially the electron and ion flux limited regime and the material (precursor) limited regime, introduced in the previous sections are very helpful to understand the "switching" in deposit properties such as electrical conductance or shape.

\section{MONTE CARLO MODELS FOR GAS ASSISTED FEB INDUCED DEPOSITION}

The strength of this approach is that all primary electron trajectories and generated secondary electrons can be traced together with their corresponding energies as they traverse any surface and material (see Fig. 33). When they encounter

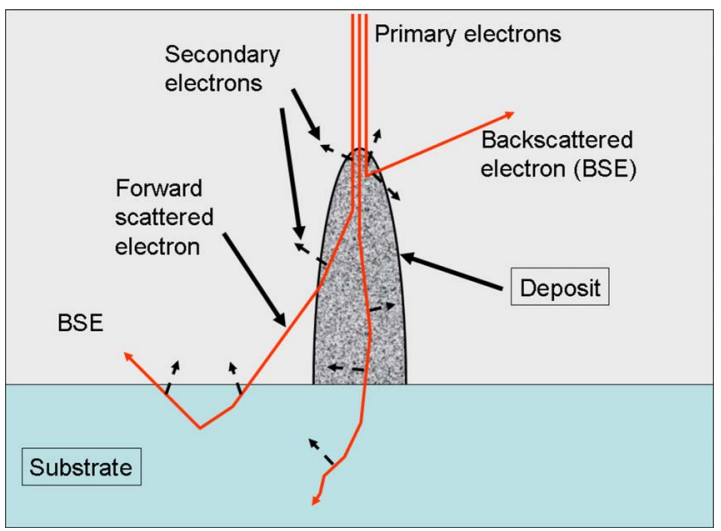

(a)

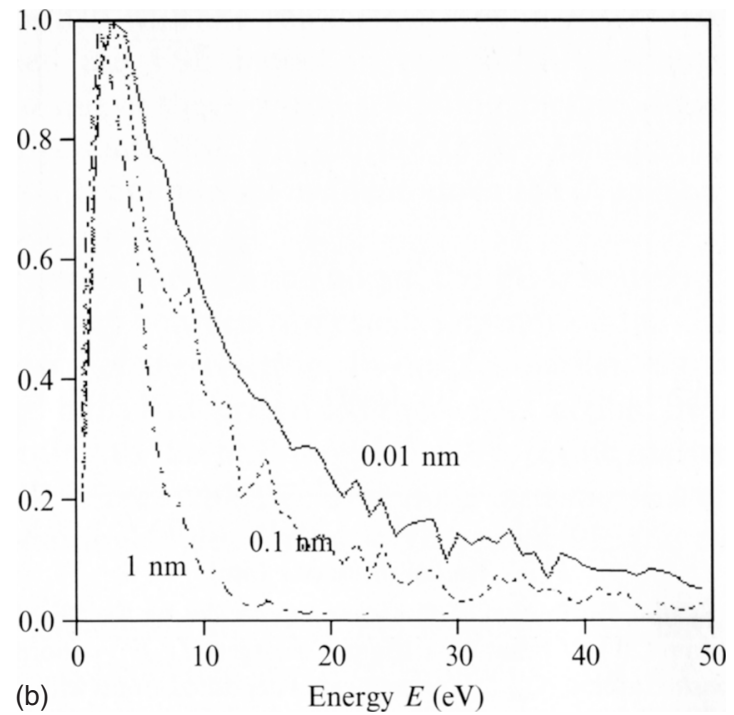

FIG. 33. (a) FEB deposition events on the surface of the deposit or substrate, which can be traced by MC trajectory simulation. (b) Simulated variation in secondary electron energy spectra (normalized) as a function of radical distance from the center of a zero diameter $20 \mathrm{keV}$ incident electron beam for a $80 \mathrm{~nm}$ thick planar $\mathrm{Cu}$ membrane (from Ref. 20).

an adsorbed molecule a reaction proceeds according to the energy dependent cross section. Presently, due to the lack of experimental data, simple generic cross sections and parametric secondary electron generation are implemented, imposing severe limits of this approach with respect to its predictive relevance for gas-assisted FEB (or FIB) experiments.

Presently, there are no $\mathrm{MC}$ simulations reported for gasassisted FIB processing. MC models for physical sputtering (without gas injection) are published (see Sec. II C 2).

\section{A. Monte Carlo models without precursor dynamics}

Monte Carlo models described in this section can be used to estimate the ultimate resolution of deposits (or etched holes) obtainable with FEB. The models assume steady state conditions and that no precursor depletion occurs, $n(r, t)$ $=n_{\text {out }}=$ const. The number of dissociated molecules per unit area and unit time becomes $f_{\text {diss }}(r)=n_{\text {out }} \int f(r, E) \sigma(E) d E$ and will determine the deposit or etch shape. Note that $R(r)$ $=V f_{\text {diss }}(r)$. Fig. 33(b) visualizes the emitted secondary elec- 
TABLE X. Summary of MC-simulated full widths containing $50 \%$ of emitted secondary electrons ( $\mathrm{FW} 50_{\mathrm{SE}}$ ) and $50 \%$ of dissociated hydrocarbon molecules $\left(\mathrm{FW} 50_{D}\right)$ (compiled from Refs. 237 and 238). Energy and size of the primary electron $(\mathrm{PE})$ beam are indicated.

\begin{tabular}{lcccc}
\hline \hline $\begin{array}{c}\text { Substrate, } \\
\text { thickness }\end{array}$ & $\begin{array}{c}\text { PE energy } \\
(\mathrm{keV})\end{array}$ & $\begin{array}{c}\text { PE diameter } \\
(\mathrm{nm})\end{array}$ & $\begin{array}{c}\mathrm{FW}^{\mathrm{FW}}{ }_{\mathrm{SE}}{ }^{\mathrm{a}} \\
(\mathrm{nm})\end{array}$ & $\mathrm{FW} 0_{D}(\mathrm{~nm})$ \\
\hline $\mathrm{Cu}, 1 \mu \mathrm{m}$ & 200 & 0 & 4.7 & 1 \\
$\mathrm{Cu}, 10 \mathrm{~nm}$ & 200 & 0 & 3.0 & 0.86 \\
$\mathrm{Cu}, 80 \mathrm{~nm}$ & 20 & 0.2 & 2.8 & $0.23^{\mathrm{b}}$ \\
$\mathrm{Cu}, 80 \mathrm{~nm}$ & 20 & 2 & 2.8 & $2^{\mathrm{b}}$ \\
\hline
\end{tabular}

${ }^{a}$ The $\mathrm{FW} 50_{\mathrm{SE}}$ is always for zero diameter PE beam.

${ }^{\mathrm{b}}$ The $\mathrm{FW} 50_{D}$ includes convolution with the PE beam diameter.

tron energy spectrum at discrete distance from a zero diameter incident beam entering into $f(r, E)$. The lateral size of the simulated emitted secondary electron flux can be characterized by the full width containing $50 \%$ of the distribution (FW50). Table X compares the FW50 of the emitted secondary electron flux with the FW50 of the deposit [being equivalent to the FW50 of $f_{\text {diss }}(r)$ defined above].

Several interesting observations can be noted from Table $\mathrm{X}$.

(1) For zero diameter incident electron beams $\mathrm{FW} 50_{\mathrm{SE}}$ $>$ FW $50_{D}$, which is a direct result of the generic cross section energy dependence used in Refs. 237 and 238. This highlights the need for accurately measured energy dependencies of cross sections for relevant molecules used in FEB or FIB processing in order to obtain reliable values for ultimate resolution.

(2) The FW50 for membrane and "bulk" substrates is very close and points to the fact that membranes must not necessarily be used for high resolution deposits. ${ }^{237}$ Using a $0.2 \mathrm{~nm}$ diameter beam results in a $\mathrm{FW}_{50}$ $=0.23$, i.e., the contribution of the emitted secondary energy flux to resolution loss is only $15 \%$ or, in other words, for the $2 \mathrm{~nm}$ diameter beam the deposit resolution is dominated by the primary electron beam size. This observation validates the applicability of the continuum approach and the scaling laws in Sec. III A down to the nanometer scale.

MC simulations show the radial growth of cylindrical structures (pillars) especially its saturation into a final cylinder diameter with increasing exposure time (see Fig. 34). Lateral growth occurs due to SE scattering in the freshly deposited structure, ${ }^{238}$ giving an ultimate resolution limit in FEB processing for the desired aspect ratio of a deposit or etch structure. A resolution of around $1 \mathrm{~nm}$ was observed for dots with an assumed aspect ratio of $0.5 .^{92}$

While in FEB contamination deposits emitted secondary electrons are exclusively responsible for the pillar shape evolution, ${ }^{238}$ it is concluded that for $\mathrm{W}$ deposition with $\mathrm{WF}_{6}$ molecules, primary electrons determine the deposition rate whereas the secondary electrons determine the pillar volume evolution. ${ }^{66}$ The reason for the difference between both simulations resides in the different decay of the dissociation cross sections with energy. This highlights the sensitivity and

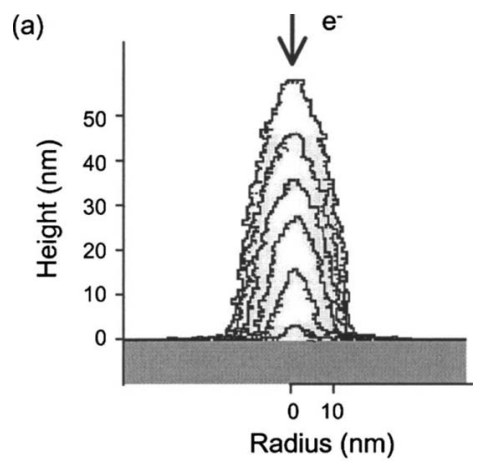

(b)

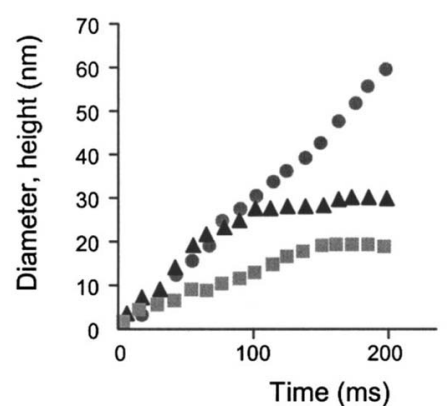

FIG. 34. (a) Sequence of simulated profiles of a contamination pillar grown by a $200 \mathrm{keV}$, zero diameter PE beam on a $10 \mathrm{~nm}$ carbon foil. (b) Evolution of pillar geometry: height (circles), base diameter (triangles), and diameter at half its height (squares) (from Ref. 238). Of note is that the apex diameter is constant and about $6 \mathrm{~nm}$.

limits of the MC simulation in predicting shapes: as long as there are no reliable data on the dominant deposition channel(s) and the energy dependence of its cross section, such simulations should not be overinterpreted. Furthermore, MC simulations neglecting precursor depletion and replenishment fail to predict a decreasing deposition rate with increasing exposure time (increasing pillar length), which is generally observed, e.g., Refs. 239 and 240. For the same reason the prediction of the correct pillar apex shape is not possible, except for the electron-limited regime.

Experimental work related to ultimate resolution deposits can be found in Refs. 186 and 241-250.

\section{B. Monte Carlo models with precursor dynamics}

Additionally to the solution of the energy integral in Eq. (2.1a), these models solve the adsorption rate equation [Eq. (2.2)] on the surface of the deposit or etch hole. This can be achieved by a full MC approach ${ }^{251}$ or by a combination of $\mathrm{MC}$ trajectory simulation and a curvilinear transformation of Eq. (2.2). ${ }^{252}$ These approaches allow for high-aspect ratio structure modeling as opposed to the continuum models discussed in Sec. III.

Figure 35 shows a recently published simulation of pillar shapes in different FEB process regimes. It confirms the trend of resolution loss with transition from electron-limited to precursor-limited regimes (increasing depletion), which was also found with the continuum approach (see Fig. 27). MC simulations additionally output the contribution of secondary electron to lateral deposit growth, which becomes 


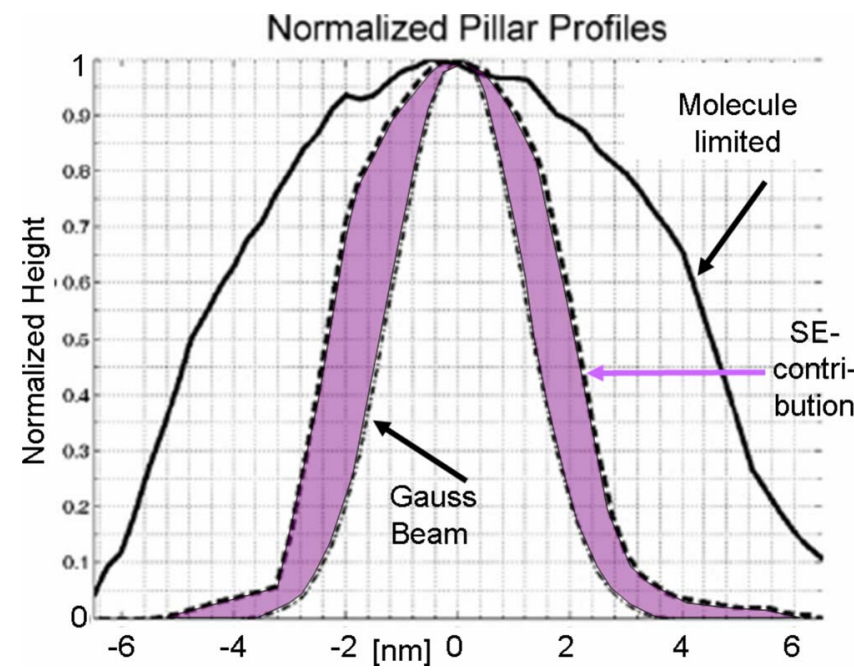

FIG. 35. MC-simulated pillar shapes $(12 \mathrm{~nm}$ tall) for differing deposition regimes including precursor kinetics (precursor: $\mathrm{WF}_{6}$ ) (adapted from Ref. 251). The secondary electron contribution to deposition for the electronlimited regime is highlighted (without secondary electrons the pillar would have the Gaussian shape of the beam). The molecule-limited regime leads to loss of deposit resolution due to depletion in the central irradiated area. The FWHM of the incident $1 \mathrm{keV}$ Gaussian electron beam is $2.7 \mathrm{~nm}$.

important for small incident beam sizes. In Fig. 35 the secondary electron emission from the pillar surface leads to around $0.8 \mathrm{~nm}$ deposited thickness along the sides of the deposits in addition to the Gaussian beam shape. This means an increase in the FWHM diameter for the deposit by about $50 \%$ (for an aspect ratio of about 4 ). Of course, the larger the incident beam size, the less important becomes the relative contribution of the secondary electron contribution to the FWHM deposit diameter, so that the scaling laws in Eqs. (3.4), (3.7), (3.8), and (3.12) can be applied also to structures having a higher aspect ratio than 1 .

Another important result these MC simulations can supply is the decay of vertical pillar deposition rates with time, i.e., with increasing pillar length. At least two reasons can be found for this dependence: increased molecule desorption due to beam heating (see Sec. II F 2) or decreasing diffusion. Since the surface diffusion changes from two dimensional (planar substrate) during the initial stages of deposition (or etching) to one dimensional (pillar mantle), the amount of molecule diffusion to the tip apex reduces. Converting the time dependence of the vertical deposition rate into a dependence on pillar length $R(L)$ makes experiments and simulations comparable. Differing relations are obtained from MC simulations considering diffusion: the relation $R(L) \sim L^{\approx-0.4}$ was simulated for $\mathrm{WF}_{6}$, etc. [from Fig. 10(c) in Ref. 251] and the relation $R(L) \sim L^{\approx-0.2}$ was simulated for hydrocarbons; ${ }^{252}$ however, both references miss a comparison to experiments. Evidently, each experiment (or simulation) with a specific parameter set (cross section, residence time, diffusion, beam size, thermal conductivity of the pillar, etc.) will result in a differing relation (see, for instance, the experimental results of Fig. 36, where power laws with exponents ranging from -0.47 to -0.88 were found).

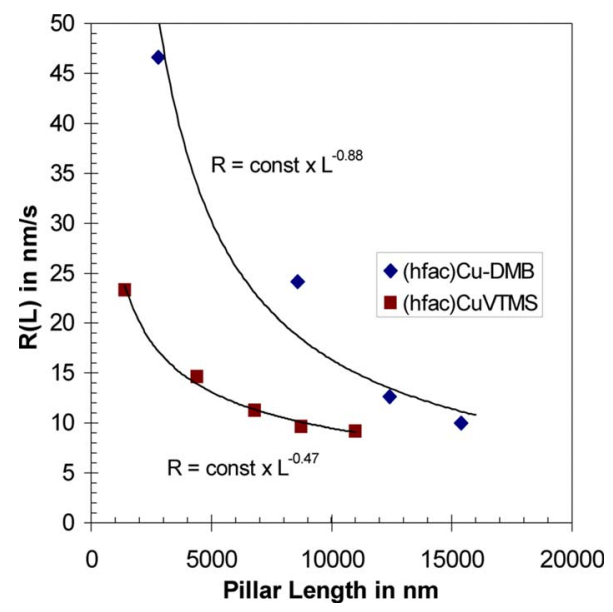

FIG. 36. Experimental relation of vertical deposition rate $R$ vs pillar length $L$ obtained from data of Ref. 240. Deposition with $25 \mathrm{keV}$ and $500 \mathrm{pA}$ electron beam and two copper precursors. The data were fitted with a power law.

Summarizing, the development of MC models with gas kinetics is still very recent. When the enormous lack of data on the adsorption, desorption, scattering, and dissociation behavior of relevant molecules can be overcome, they have the potential to derive scaling laws for the pillar apex and cylinder geometry as a function of the incident electron energy and the degree of molecule depletion. For a comprehensive overview on numerous experimental findings on tip geometries and deposition rates, see a recent review ${ }^{253}$ and Refs. 239 and 254-258.

\section{PRECURSOR MOLECULES}

The precursors for FEB and FIB induced processing (deposition and etching) are of paramount importance. Therefore we dedicated this section to an overview of the applied precursors ordered according to the precursor ligands. For deposition of metals, chemical vapor deposition precursors are often applied, and for a general overview of the thermal and photolytic chemical vapor deposition literature reviewed until 1994, we refer to Kodas and Hampden-Smith. ${ }^{168}$ We include and discuss the data from the lists in already published review papers. ${ }^{29,259}$ The choice of precursor gas determines what material is deposited or how a material is etched. The precursor gases for FIB and FEB induced reactions are the same: ideally, they should stick to and reside long on the surface to be activated/dissociated for etching or for deposition. On the other hand, the volatile compound formed in the etching process should desorb readily, as well as the nondesired fragments formed upon irradiation of precursors for decomposition. Since the ligands originate from the same "mother" molecule that should be reasonably volatile, these two requirements are conflictive, meaning that an optimum must be found. 


\section{A. General aspects}

\section{The role of residual molecules in microscope chambers}

The influence of other molecules present in the system has often been overlooked. Herein water plays a key role, and then follows hydrocarbons. As these two contaminants are in most cases not measured nor estimated, the results of deposition with any precursor will strongly be influenced by the presence of these gases. For focused electron beam induced etching (FEBIE), the presence of water might strongly enhance the process; the presence of carbonaceous contaminants on the other hand will completely stop the process. Already early work of FEBID pointed out the importance of vacuum quality that explained the limited purity of the obtained deposits. ${ }^{9}$ Exemplary sample cleaning and microscope cleaning processes are presented by Crozier's group from the Arizona State University ${ }^{260}$ as described in the report about deposition of germanium ${ }^{261}$ and the UHV electron microscope studies by Tanaka and Furuya. ${ }^{186}$ For most of the shown results in this section, however, the influence of water (or with lower importance hydrocarbons) on the deposition process should be kept in mind. Water, being the main residual gas component in SEMs and SIMs, ${ }^{262}$ is also known to chemisorb on certain surfaces and can then only be removed by baking. In a recent study the water desorption behavior of stainless steel and aluminum surfaces are studied in air and vacuum. ${ }^{263}$ It was found that water desorption from stainless steel and aluminum surfaces in vacuum is slower than in atmosphere with the residual coverage described approximately by a $1 / t^{\alpha}$ law, with $\alpha \approx 0.14$ for stainless steel and $\alpha \approx 0.17$ for Al. Starting from a monolayer of water $\left(\approx 10^{15}\right.$ molecules $/ \mathrm{cm}^{2}$, see Table VI) adsorbed on stainless steel at room temperature, it will take 2 min to decrease to half of a monolayer, but 160 days to reduce to one-tenth of a monolayer. As most of the vacuum chambers of microscopes posess more than $1000 \mathrm{~cm}^{2}$ of surface, there are $10^{15}-10^{17}$ molecules of water desorbing from the walls during most processing cycles. The omnipresence of water is confirmed with mass spectrometers such as residual gas analyzers attached to electron microscopes representing in most cases more than half of the residual gas in the unheated vacuum systems pumped down to $10^{-6} \mathrm{mbar}$ total pressure. According to Sec. II D 1 this corresponds to an impinging flux of water molecules of roughly $0.1 \mathrm{ML} / \mathrm{s}$ or $10^{14}$ molecules $/ \mathrm{cm}^{2} \mathrm{~s}$.

\section{Precursor stability}

After synthesis purification of the compound has to be carried out in order to minimize potential contaminants. During storage transformation of compounds might take place. Important parameters influencing the degree of degradation of compounds are the container materials (stainless steel, glass, aluminum, and polymers), the storage time, and the temperature. Furthermore for halogens or halogen containing gases, mechanical shocks of the metal containers should be avoided because of metal halogenide particles detaching from the metal walls of the containers. As an example the storage of iron carbonyl $\mathrm{Fe}(\mathrm{CO})_{5}$ in a closed container might decompose partially to result in higher clustering by exposure to UV light, ${ }^{264}$ ultrasonication, ${ }^{265}$ or thermal decomposition to pure iron particles by heating up the container. Interesting is the fact that the products of the different exposure types are different compounds. Light exposure of $\mathrm{Fe}(\mathrm{CO})_{5}$ adsorbed on silica (for example, a glass container) results in the trinuclear complex $\mathrm{Fe}_{3}(\mathrm{CO})_{12}$, whereas irradiation in the gas phase or in liquid solution results in $\mathrm{Fe}_{2}(\mathrm{CO})_{9}$ the dinuclear compound.

Pumping at room temperature on precursors might change the chemical composition of the substance by slow but constant ligand loss and therefore higher clustering. Another phenomenon, the loss of one water ligand, has been observed for the hexafluoroacetylacetonato-copper precursor, the light green $\mathrm{Cu}(\mathrm{hfa})_{2} \cdot \mathrm{H}_{2} \mathrm{O}$. This air stable compound has a different vapor pressure and evaporation enthalpy than the dried water-free blue gray compound $\mathrm{Cu}(\mathrm{hfa})_{2} \cdot{ }^{266}$ The water-free compound can be obtained by pumping at room temperature on the precursor reservoir. Clustering of precursors with or without ligand loss is postulated for several precursor cases, but not easily measured.

\section{Vapor pressure and evaporation enthalpy}

The precursor's vapor pressure is an important property of a compound as it determines the amount of molecules evaporated from a precursor reservoir to be transported to the substrate. Furthermore, the evaporation enthalpy of a compound is an important property for FEB and FIB induced deposition and etching because it is a measure for the energetics of adsorption and desorption phenomena of those compounds on substrates. Determination of the gas phase composition (vapor pressure) of volatile compounds (precursors) and the potential residue is often carried out by thermo gravimetrical analysis. The dynamic regime applying a carrier gas does not necessarily result in identical results as the pure pumping on a precursor. Alternative methods for measuring vapor pressures and evaporation enthalpies were applied also, resulting in different values. Chickos and Acree collected and tabulated a large number of vapor pressure measurements and evaporation and sublimation enthalpies published in the past 100 years. ${ }^{267,268}$ They emphasize the variations of the values, especially for the evaporation/sublimation enthalpy that might vary by more than one order of magnitude due to polymorphism and insufficient measurement accuracy. ${ }^{268}$ For the evaporation enthalpy of $\mathrm{Al}(\mathrm{acac})_{3}$, for example, values can be found in literature ranging from 43.7 to 126 $\mathrm{kJ} / \mathrm{mol}^{266}$

\section{B. Complexes for deposition}

This section is organized in the following way. A special subsection is dedicated to the deposition of carbon because it has some interesting peculiarities. The classification takes place according to the ligands around the central atom of the molecules for "deposition" precursors. The simplest ligands are hydrogen $\mathrm{H}$ or the halogens $\mathrm{F}, \mathrm{Cl}, \mathrm{Br}$, and $\mathrm{I}$. If on one central atom there are different ligands, a so-called hetero- 
leptic complex is formed. These mixed ligand complexes are classified here always in the class of the more difficult to get rid of ligand. We present also compounds composed of an uncharged central atom with four or more molecular uncharged ligands such as $\mathrm{CO}$ and $\mathrm{PF}_{3}$. Then heteroleptic compounds with low charged central atoms neutralized with halogeno ligands and $\mathrm{CO}$ and $\mathrm{PF}_{3}$ follow. Further some metal organic complexes are mentioned, especially compounds that where used for FEBID by adding reactive gases. In a final part of deposition compounds, the post-treatment of deposits are discussed briefly.

Generally precursors are complexes composed of central atoms/ions and ligands. To be volatile, the precursor complex must be uncharged, not possessing a strong dipole moment, and not be too easily polarizable. High sticking probability on a surface would need the opposite properties. Purely empirically it turns out that deposition processes of neutral or low charged central atoms result in higher metal contents than highly charged central ions. The need to reduce the central ion in the deposition processes for obtaining pure metals could be interpreted to be a limiting factor. However, the electrostatic bond strength that increases the stability of highly charged central ion complexes is a much better argument. However, finally the presence of elements that easily polymerize like carbon seems to be the most important argument. The latter reasoning is based on the fact that the charged particle beam induced deposition process (FEBID/ FIBID) is accompanied with unspecific multiple site fragmentation of adsorbed precursor molecules. The latter explains the easy desorption of recombining atoms of volatile elements, but the unsuccessful desorption of elements that are solids in their elementary form such as carbon, phosphorus, and metals. Pure materials can be obtained in cases that the electrons or ions initiate a chemical reaction but do not fragment the reaction products before desorption of the volatile species. This can be obtained at relatively low electron densities and with precursors that dissociate easily (at low temperature or autocatalytically) or that a chemical reaction such as an oxidation is initiated by the electrons. Further detailed analysis of these thoughts should be carried out in the future.

\section{Organic compounds for $C$ deposition}

Carbon deposits are the most common FEBID products as they appear as contamination results in electron microscopy. From a chemist point of view, alkanes $\left(\mathrm{C}_{n} \mathrm{H}_{2 n+2}\right)$ are nonpolar relatively inert molecules that do not undergo chemical reactions at room temperature. The small alkanes from $n=1$ to 5 are gases, from hexane $(n=6)$ to heptadecane $(n=17)$, decreasingly volatile liquids, and from octadecane $\left(\mathrm{C}_{18} \mathrm{H}_{38}\right)$ to higher alkanes, solids. Branched alkanes are alkanes with the same ratio of $\mathrm{C}$ and $\mathrm{H}$ but $Y$ junctions or crosses in the $\mathrm{C}-\mathrm{C}$ backbone chains that possess slightly lower boiling points than the $n$-alkanes. Generally mineral oils are mixtures of liquid and solid linear and branched alkanes. Synthetic oils are divided in two groups, the polyphenylethers that are aromatic phenyl rings linked with $\mathrm{O}$ bridges
$\mathrm{C}_{6} \mathrm{H}_{5}-\left(-\mathrm{O}-\mathrm{C}_{6} \mathrm{H}_{4}-\right)_{n}-\mathrm{O}-\mathrm{C}_{6} \mathrm{H}_{5}$ and silicone oils $\left.\left(\mathrm{H}_{3} \mathrm{C}\right)_{3} \mathrm{Si}-\left(-\mathrm{O}-\mathrm{Si}\left(\mathrm{CH}_{3}\right) \mathrm{C}_{6} \mathrm{H}_{5}\right)-\mathrm{O}-\right)_{n}-\mathrm{Si}\left(\mathrm{CH}_{3}\right)_{3}$ that are chemically completely different as they contain an- $\left(-\mathrm{O}_{-}\right.$ $\mathrm{Si}-)$-backbone chain that reacts at high temperatures with oxygen to $\mathrm{SiO}_{2}$, a solid. The silicone oils will be discussed with the $\mathrm{Si}$ containing precursors. The contamination of vacuum systems depends on a variety of factors (type of pumps and lubricants used) and therefore no details are reported in literature about the exact chemical composition or exposure rate by such compounds. Furthermore the pump oil suppliers do not specify the exact composition of their products. The vapor pressures of the different compounds range from $<10^{-9}-10^{-3} \mathrm{mbar}$ at room temperature. Continuous pumping on such compounds changes the chemical composition continuously by evaporating more volatile compounds at higher rate. Cracking and partial oxidation of the oils are determined by the temperature, the contact surfaces, exposed gases (air, water, halogens, etc.), and traces of metals dissolved in the oil that might act as catalysts for chemical reactions. The degradation products of mineral oils or synthetic pure organic oils are organic compounds either saturated (only single bonds) or partially unsaturated, or aromatic systems, including oxidation products such as alcohols, ketones, aldehydes, or organic acids. Such systems are not ideal for studying FEBIP parameters.

For this reason the systematic study of selected precursors for $\mathrm{C}$ deposition that has recently been carried out ${ }^{190,269}$ was needed. The list of tested organic precursors is tabulated in Table XI. The most important message from Bret's work is that FEBID of organic compounds including organic acids such as acetic acid, formic acid, maleic acid, through aldehydes and ketones, alcohols to hydrocarbons, and unsaturated aromatic precursors all result in the same final composition of $\mathrm{C}_{9} \mathrm{H}_{2} \mathrm{O}$ in a classical dry pumped scanning electron microscope without any special care about residual water contamination. The difference of the precursors is mainly the growth rate of the deposit that could be explained by the different residence times of the precursors on the substrate surface. The simplest alkane is methane. The latter was used together with hydrogen gas for deposition of conductive emitters of carbon. ${ }^{270}$ The low deposition rate of $1 \mu \mathrm{m}^{3}$ in $30 \mathrm{~min}$ is due to the low sticking coefficient of the methane on the substrate and partially to the etching of carbon in the presence of hydrogen that might have taken place. Heating a silicon substrate to $800{ }^{\circ} \mathrm{C}$ under electron irradiation in the presence of $1 \%$ of methane in hydrogen results in local deposition of diamond. ${ }^{271}$ Methyl-isobutylketone with isopropanol in a ratio 1:3 was applied to do FEBID of $C^{259}$ In this context styrene as a precursor was used by $\mathrm{Ochiai}^{272}$ and a deposition yield of $0.001 \mathrm{C}$ atoms per electron was obtained. This was lower than deposits from other metalorganic precursors and explained by the authors with the low sticking coefficient of styrene on the substrate surface. Of note is that, often the deposition rate is given in units of volume per incident charge (see Table XI). When converting into yields 
TABLE XI. FEB and FIB induced deposition of carbon containing material. The precursors, vapor pressures $P_{\text {vap }}$, deposition rates, and the deposition

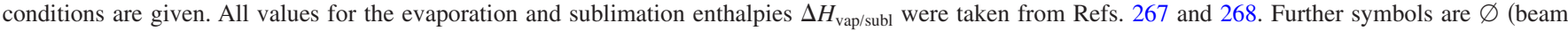
diameter), $P_{\text {res }}$ (residual vacuum pressure), and $P_{\text {dep }}$ [back pressure during deposition or estimated local pressure on sample surface (mbar)].

\begin{tabular}{|c|c|c|c|c|c|c|}
\hline Precursor & $\begin{array}{l}\text { Compound } \\
\text { name }\end{array}$ & $\begin{array}{l}P_{\text {vap }} \\
\text { (mbar) } \\
\text { at RT }\end{array}$ & $\begin{array}{c}\Delta H_{\mathrm{vap} / \mathrm{subl}} \\
(\mathrm{kJ} / \mathrm{mol})\end{array}$ & $\begin{array}{l}\text { Dep. rate } \\
\left(\mu \mathrm{m}^{3} \mathrm{nA}^{-1} \min ^{-1}\right)\end{array}$ & $\begin{array}{l}\text { Deposition } \\
\text { conditions }\end{array}$ & Ref. \\
\hline $\mathrm{CH}_{4}$ & Methane & $\begin{array}{l}45900 \text { at } T_{\text {crit }} \\
\left(-82.7^{\circ} \mathrm{C}\right)^{\mathrm{a}}\end{array}$ & $8.6(175 \mathrm{~K})$ & $\begin{array}{l}3.3 \\
0.1 \mu \mathrm{m} / \mathrm{h}\end{array}$ & $\begin{array}{l}4 \mathrm{keV} ; 10^{-11} \mathrm{~A},<0.3 \mu \mathrm{m} \varnothing ; \\
10 \mathrm{keV} ; 30 \mathrm{nA}, \\
2.6 \mu \mathrm{m} \varnothing ;\end{array}$ & 270 and 271 \\
\hline $\mathrm{C}_{2} \mathrm{H}_{4}$ & Ethylene & $\begin{array}{l}50760 \text { at } T_{\text {crit }} \\
\left(-9.5^{\circ} \mathrm{C}\right)^{\mathrm{a}}\end{array}$ & $14.0(267 \mathrm{~K})$ & $\cdots$ & $\begin{array}{l}P_{\text {res }}=5 \times 10^{-10} \mathrm{mbar} \\
2 \mathrm{keV} ; 400 \mathrm{~mA} / \mathrm{cm}^{2} \\
300 \mu \mathrm{m} \varnothing \\
5.7 \times 10^{-2} \mathrm{C} / \mathrm{cm}^{2} \mathrm{~s} ; 400 \mathrm{kV} \\
4 \times 10^{-4} \mathrm{C} / \mathrm{cm}^{2} \mathrm{~s}\end{array}$ & 274 and 275 \\
\hline $\mathrm{C}_{3} \mathrm{H}_{8}$ & Propane & $8700^{\mathrm{a}}$ & $18.8(293 \mathrm{~K})$ & $\cdots$ & $\begin{array}{l}P_{\text {dep }}=1.3 \times 10^{-4} \\
10^{-8} \text { Torr; } \\
1 \mathrm{keV} \text { and } 6 \mu \mathrm{A} \\
3 \mathrm{kV} \text { and } 35 \mu \mathrm{A}\end{array}$ & 276 \\
\hline $\mathrm{C}_{8} \mathrm{H}_{8}$ & Styrene & 6.65 & $\begin{array}{l}43.5 \pm 0.4 \\
(298 \mathrm{~K})\end{array}$ & $\begin{array}{l}(0.001 \\
\text { atom/electron }) \\
0.05 \mathrm{~nm} /(\mu \mathrm{C} / \mathrm{cm})\end{array}$ & $\begin{array}{l}P_{\text {dep }}=1.33 \times 10^{-5} \\
P_{\text {res }}=10^{-9} \text { Torr; } \\
50 \mathrm{keV} ; 1000 \mathrm{~mA} / \mathrm{cm}^{2}, \\
11 \mathrm{~nm} \varnothing ; 1 \mathrm{nA}\end{array}$ & 272 \\
\hline $\mathrm{C}_{8} \mathrm{H}_{8}$ & Styrene & 6.65 & Same & 0.021 & $\begin{array}{l}P_{\text {dep }}=4.5 \times 10^{-5} \\
3.1 \times 10^{19} \text { molecules } / \mathrm{s} \\
25 \mathrm{keV}, 2-5 \mathrm{nA}\end{array}$ & 190 and 269 \\
\hline $\mathrm{C}_{10} \mathrm{H}_{16} \mathrm{O}$ & Camphor & 0.65 & $54.4-55.2(298 \mathrm{~K})$ & 0.0324 & $\begin{array}{l}P_{\text {dep }}=3 \times 10^{-5} \\
25 \mathrm{keV}, 2-5 \mathrm{nA}\end{array}$ & 269 \\
\hline $\mathrm{C}_{10} \mathrm{H}_{20} \mathrm{O}$ & Menthol & $1\left(20^{\circ} \mathrm{C}\right)$ & $58.2(344 \mathrm{~K})$ & 0.034 & $\begin{array}{l}P_{\mathrm{dep}}=2 \times 10^{-5} \\
25 \mathrm{keV}, 2-5 \mathrm{nA}\end{array}$ & 269 \\
\hline $\mathrm{C}_{7} \mathrm{H}_{6} \mathrm{O}_{2}$ & $\begin{array}{l}\text { Benzoic } \\
\text { acid }\end{array}$ & $1 \times 10^{-3}-5.32 \times 10^{-3}$ & $78.9(289 \mathrm{~K})$ & $<0.013$ & $\begin{array}{l}P_{\mathrm{dep}}=1.5 \times 10^{-5} \\
25 \mathrm{keV}, 2-5 \mathrm{nA}\end{array}$ & 269 \\
\hline $\mathrm{C}_{2} \mathrm{H}_{6} \mathrm{SO}$ & $\begin{array}{l}\text { Dimethylsulfoxide } \\
\text { (DMSO) }\end{array}$ & 0.6 & $52.4(308 \mathrm{~K})$ & 0.014 & $\begin{array}{l}P_{\mathrm{dep}}=3 \times 10^{-5} \\
25 \mathrm{keV}, 2-5 \mathrm{nA}\end{array}$ & 269 \\
\hline $\mathrm{C}_{6} \mathrm{H}_{12}$ & Cyclohexane & 100 & $32.9(298 \mathrm{~K})$ & 0.01 & $\begin{array}{l}P_{\mathrm{dep}}=7 \times 10^{-4} \\
25 \mathrm{keV}, 2-5 \mathrm{nA}\end{array}$ & 269 \\
\hline $\mathrm{C}_{6} \mathrm{H}_{10} \mathrm{O}$ & Cyclohexanone & 11.3 & $45.1-46.9(298 \mathrm{~K})$ & $<0.007$ & $\begin{array}{l}P_{\mathrm{dep}}=7 \times 10^{-4} \\
25 \mathrm{keV}, 2-5 \mathrm{nA}\end{array}$ & 269 \\
\hline $\mathrm{C}_{9} \mathrm{H}_{19}-\mathrm{CN}$ & Cyanononane & $\cdots$ & $\begin{array}{l}66.8 \pm 0.4 \\
(298 \mathrm{~K})\end{array}$ & $<0.012$ & $\begin{array}{l}P_{\text {dep }}=7 \times 10^{-5} \\
25 \mathrm{keV}, 2-5 \mathrm{nA}\end{array}$ & 269 \\
\hline $\mathrm{CH}_{2} \mathrm{Cl}_{2}$ & Dichloromethane & 447 & $30.6(298 \mathrm{~K})$ & 0.019 & $\begin{array}{l}P_{\text {dep }}=7 \times 10^{-4} \\
25 \mathrm{keV}, 2-5 \mathrm{nA}\end{array}$ & 269 \\
\hline $\mathrm{C}_{3} \mathrm{H}_{6} \mathrm{O}$ & Acetone & 526 & $31.9(300 \mathrm{~K})$ & 0.04 & $\begin{array}{l}P_{\text {dep }}=7 \times 10^{-4} \\
25 \mathrm{keV}, 2-5 \mathrm{nA}\end{array}$ & 269 \\
\hline $\mathrm{CH}_{3} \mathrm{OH}$ & Methanol & 128 & $37.3(298 \mathrm{~K})$ & 0.014 & $\begin{array}{l}P_{\mathrm{dep}}=7 \times 10^{-4} \\
25 \mathrm{keV}, 2-5 \mathrm{nA}\end{array}$ & 269 \\
\hline $\mathrm{CH}_{3} \mathrm{CN}$ & Acetonitrile & 96 & $33(298 \mathrm{~K})$ & 0.034 & $\begin{array}{l}P_{\mathrm{dep}}=7 \times 10^{-4} \\
25 \mathrm{keV}, 2-5 \mathrm{nA}\end{array}$ & 269 \\
\hline $\mathrm{CH}_{3} \mathrm{NO}_{2}$ & Nitromethane & 37 & $\begin{array}{l}37.2-38.3 \\
(298 \mathrm{~K})\end{array}$ & 0.034 & $\begin{array}{l}P_{\mathrm{dep}}=8 \times 10^{-4} \\
25 \mathrm{keV}, 2-5 \mathrm{nA}\end{array}$ & 269 \\
\hline $\mathrm{HCOOH}$ & Formic acid & 46 & $\begin{array}{l}19.9-46.3 \\
(298 \mathrm{~K})\end{array}$ & 0.02 & $\begin{array}{l}P_{\mathrm{dep}}=8 \times 10^{-5} \\
6.1 \times 10^{20} \text { molecules } / \mathrm{s} \\
25 \mathrm{keV}, 2-5 \mathrm{nA}\end{array}$ & 190 \\
\hline $\mathrm{CH}_{3}-\mathrm{COOH}$ & Acetic acid & 15 & $\begin{array}{l}23.3-51.6 \\
(298 \mathrm{~K})\end{array}$ & 0.021 & $\begin{array}{l}P_{\text {dep }}=5 \times 10^{-5} \\
2 \times 10^{20} \text { molecules } / \mathrm{s} \\
25 \mathrm{keV}, 2-5 \mathrm{nA}\end{array}$ & 190 \\
\hline $\mathrm{CH}_{3} \mathrm{CH}_{2}-\mathrm{COOH}$ & $\begin{array}{l}\text { Propionic } \\
\text { acid }\end{array}$ & $\begin{array}{l}4.4 \\
\left(27.6{ }^{\circ} \mathrm{C}\right)\end{array}$ & $\begin{array}{l}31.1-55 \\
(298 \mathrm{~K})\end{array}$ & 0.019 & $\begin{array}{l}\mathrm{P}_{\mathrm{dep}}=4 \times 10^{-6} \\
7 \times 10^{18} \text { molecules } / \mathrm{s} \\
25 \mathrm{keV}, 2-5 \mathrm{nA}\end{array}$ & 190 \\
\hline $\mathrm{CH}_{2}=\mathrm{H}-\mathrm{COOH}$ & Acrylic acid & 3.8 & $\begin{array}{l}53.1 \pm 4.2 \\
(298 \mathrm{~K})\end{array}$ & $0.076-0.112$ & $\begin{array}{l}\mathrm{P}_{\mathrm{dep}}=5 \times 10^{-5} \\
3.5 \times 10^{19} \text { molecules } / \mathrm{s} \\
25 \mathrm{keV}, 2-5 \mathrm{nA}\end{array}$ & 190 \\
\hline $\mathrm{C}_{14} \mathrm{H}_{10}$ & Phenanthrene & & & $\begin{array}{l}\text { Total yield: } 8 \\
\text { atoms/ion }\end{array}$ & $\begin{array}{l}3.8 \times 10^{8} \mu \Omega \mathrm{cm}, \\
600 \mathrm{GPa} \\
\text { (Young's } \\
\text { modulus) }\end{array}$ & 277 and 36 \\
\hline
\end{tabular}

ahttp://encyclopedia.airliquide.com 
TABLE XII. FEB and FIB induced deposition using hydride precursors. The precursors, vapor pressures $P_{\text {vap}}$, deposition rates, and the deposition conditions are given. Other symbols are $\varnothing$ (beam diameter), $P_{\text {res }}$ (residual vacuum pressure), $P_{\text {dep }}$ (pressure during deposition or estimated local pressure on sample surface), and $t_{\text {dep }}$ (deposition time).

\begin{tabular}{|c|c|c|c|}
\hline $\begin{array}{l}\text { Precursor } \\
\text { irradiation }\end{array}$ & $\begin{array}{l}P_{\text {vap }} \\
(\mathrm{mbar})\end{array}$ & Conditions & $\begin{array}{l}\text { Refs. and } \\
\text { remarks }\end{array}$ \\
\hline $\begin{array}{l}\mathrm{Si}_{2} \mathrm{H}_{6} \\
500 \mathrm{eV} \text { electrons }\end{array}$ & $3300^{\mathrm{a}}$ & $\begin{array}{l}\mathrm{Si}(100) 2 \times 1 \text { reconstructed, } t_{\mathrm{dep}}: 300 \mathrm{~s}, \\
12 \mu \mathrm{A} \text {. }\end{array}$ & 281 \\
\hline $\begin{array}{l}\mathrm{Si}_{2} \mathrm{H}_{6} \\
5 \mathrm{keV} \text { electrons }\end{array}$ & $3300^{\mathrm{a}}$ & $\begin{array}{l}\mathrm{Si}, \mathrm{Ge} \text { preheated to } 1200 \text { and } 600{ }^{\circ} \mathrm{C} \\
\text { for } 1 \mathrm{~min} \text { before deposition; } \\
P_{\text {res }}: 4 \times 10^{-10} \text { Torr, } P_{\mathrm{dep}}: 2 \times 10^{-5} \mathrm{Torr} \text {; } \\
7 \mu \mathrm{A}, 3 \mathrm{~mm} \varnothing ; 400 \mathrm{~min} \text {, in situ Auger. }\end{array}$ & $\begin{array}{l}\text { All system } \\
\text { bakeable; heated } \\
\text { for } 24 \mathrm{~h} \text {, } \\
\text { (Ref. 279) }\end{array}$ \\
\hline $\begin{array}{l}\mathrm{Ge}_{2} \mathrm{H}_{6} \\
200 \mathrm{keV} \text { electrons }\end{array}$ & $737^{\mathrm{b}}$ & $\begin{array}{l}\mathrm{Si}_{3} \mathrm{~N}_{4} \text { membranes, } P_{\text {res: }}: 10^{-7} \text { Torr, } P_{\mathrm{dep}}: \\
10^{-5} \text { Torr, } 1-5 \mathrm{pA}, 1-4 \mathrm{~nm} \varnothing ; t_{\mathrm{dep}}: 12 \mathrm{~min} \text {, } \\
\text { EELS }\end{array}$ & 261 \\
\hline $\begin{array}{l}\mathrm{D}_{2} \mathrm{GaN}_{3} \\
200 \mathrm{keV} \text { electrons }\end{array}$ & $\begin{array}{l}0.27 \text { at } \\
40{ }^{\circ} \mathrm{C}^{\mathrm{c}}\end{array}$ & $\begin{array}{l}\text { Thinned Si with amorphous oxide layer, } \\
\text { heated to } 70^{\circ} \mathrm{C}, P_{\text {res }}: 2 \times 10^{-7} \text { Torr, } P_{\text {dep }} \text { : } \\
10^{-5} \text { Torr, } 20 \mathrm{pA}, 0.2 \mathrm{~nm} \varnothing, t_{\text {dep }}: 12 \mathrm{~min} \text {, } \\
\text { EELS }\end{array}$ & 185 \\
\hline
\end{tabular}

ahttp://encyclopedia/airliquide.com

bhttp://metaloids.com/docs/diger_msds

${ }^{c}$ References 283 and 284.

(in units of deposited atom per electron or ion) the deposit's density and molar mass should be exactly known [see Eq. (6.1)].

Another interesting aspect, a chemical argument, the polymerizability of the precursor was discussed for aromatic alternated double or triple bond containing compounds under FIBID conditions. ${ }^{273}$ Styrene deposited well, whereas benzene and toluene resulted in material removal by the focused ion beam.

During FEBID or FIBID, the precursor molecules have to adsorb on the "just deposited" material. Only at the very beginning of the deposition process, studies of the chemisorption of molecules on the ultra-clean well characterized substrates are important. Examples of such extensive works are the report about ethylene $\left(\mathrm{C}_{2} \mathrm{H}_{4}\right)$ adsorbed on $\mathrm{Si}(001)$ and irradiated with electrons from an Auger gun $(2 \mathrm{keV}, 300 \mu \mathrm{m}$ diameter) or from a heated $\mathrm{W}$ filament resulted in amorphous hydrogenated carbon deposits. ${ }^{275}$ These experiments were carried out in an UHV system with a residual pressure without precursor gas injected of $5 \times 10^{-10}$ mbar. Ethylene chemisorbs at $300^{\circ} \mathrm{C}$ on this $\mathrm{Si}(001)$ substrate until a given coverage is reached. The FEBID growth rates in the initial stage were very fast, then after having deposited some carbon, ethylene adsorbed on the just deposited carbon material and the decreased deposition rate reached a constant value.

Resuming the $\mathrm{C}$ deposition experiments reported so far, $\mathrm{C}$ can be deposited from different precursors and the residual gases present in the vacuum system. The deposit materials range from amorphous carbon to graphite; under special gas composition conditions and irradiated parameters, diamond or at least diamond like carbon (as a mixture of $\mathrm{sp}^{2}$ and $\mathrm{sp}^{3}$ hybrized Carbon) was also claimed. ${ }^{271}$

\section{Hydrides}

The only hydrides that have been studied for deposition experiments are the volatile hydrides of the group IV ele- ments, C, Si, and Ge (see Table XII). Carbon has already been discussed above. Another interesting new type of precursor Ga hydrides-azides that contains the azide as ligand is included here also, even though nitrogen might be a contaminant found as nitride in the deposit.

(a) Silicon ( $\mathrm{Si}$ ). The application of monosilane $\mathrm{SiH}_{4}$ for $\mathrm{SiO}_{2}$ deposition by parallel-to-the-substrate electron beam induced activation of the 5\% silane in argon gas with $10 \%$ $\mathrm{N}_{2} \mathrm{O}$ was realized for lowering the substrate deposition temperature. $^{278}$ This kind of gas activation is not directly comparable with FEBID due to the strong differences concerning the cross sections of molecules in the gas phase as compared to the adsorbed phase molecules. ${ }^{107}$

FEB silicon $(\mathrm{Si})$ deposition from disilane $\left(\mathrm{Si}_{2} \mathrm{H}_{6}\right)$ was reported in an UHV system with $5 \mathrm{keV}$ electrons and a very large electron beam ( $2 \mathrm{~mm}$ beam diameter). ${ }^{279}$ The study shows that surface stimulated processes are predominant as compared to gas phase reactions. Crystalline $\mathrm{Si}$ deposits were obtained on $\mathrm{Si}$ and Ge substrates. Adding oxygen to the disilane resulted in deposition of relatively good quality of $\mathrm{SiO}_{2}{ }^{280}$ The same disilane precursor was studied in depth and the dissociation into hydrogen-terminated silicon species was shown to occur in an UHV system above $700 \mathrm{~K}$ on pure Si (100) $2 \times 1$ reconstructed surfaces. ${ }^{281}$

(b) Germanium $(\mathrm{Ge}$ ). Germanium deposition from digermane $\left(\mathrm{Ge}_{2} \mathrm{H}_{6}\right)$ (Ref. 261) in a high pressure subchamber equipped environmental transmission electron microscope at $200 \mathrm{kV}$ was carried out on $\mathrm{Si}_{3} \mathrm{~N}_{4}$ membranes. The deposit content was mainly Ge as confirmed by EELS, less than $18 \%$ $\mathrm{C}$ contamination. The dimensions of the deposits were larger than the electron beam, explained by secondary electron escape depth in the deposit.

(c) Gallium nitride $(\mathrm{GaN})$. Another group of materials consists of III-V semiconductor compounds. Pure GaN FEBID was obtained by using the volatile mixed dinuclear pre- 
cursor $\mathrm{D}_{2} \mathrm{GaN}_{3} \cdot{ }^{185}$ This compound decomposes already at $150{ }^{\circ} \mathrm{C}$ to result in crystalline $\mathrm{GaN}$ as shown in an UHVCVD study. ${ }^{282}$ The precursor can be distilled at $40{ }^{\circ} \mathrm{C}$ at 200 Torr pressure and exists in the gas phase as trimer. The deposit dimensions were determined by the secondary electron escape dimensions and increase with increased deposition time.

\section{Halides}

Metal halides are toxic and corrosive and are therefore not the preferred choice of precursors for FEB induced deposition. On the other hand they can result in relatively pure deposits. As in the general case discussed before, adsorbed halogeno-precursor molecules might partially fragment on the substrate or deposit surface and the purity of the deposit will be determined by the desorption of the remaining halogen ligands. Generally the electronic excitation into an antibonding electronically excited state leads to desorption. Alternatively, thermally induced recombination of halogen ligands to diatomic halogen molecules and subsequent desorption from the surface will result in deposition of clean metals. A special example revealing the potential complexity of the deposition and/or etching processes has recently been discussed for the case of bromine desorption from $\mathrm{Si}(001)$ surfaces. ${ }^{285}$ Here the electronic excitation results in phonon excitation in the substrate that results finally in the desorption of molecular bromine. For in-depth understanding of the deposition processes of above materials, the desorption literature of halogens from these compounds should be checked.

(a) Tungsten $(W)$. Matsui tested FEBID with tungsten hexachloride $\mathrm{WCl}_{6}$ (Ref. 10) resulting in about 60 at. \% W. We think that the 18 at. \% Oxygen contamination in the deposit stem from residual water vapor. The most prominent metal halide used for FEBID is probably tungsten hexafluoride $\mathrm{WF}_{6}$. The first studies revealed very interesting deposition versus etching behaviors. The chemical reaction between $\mathrm{WF}_{6}$ and $\mathrm{Si}$ in the presence or absence of $\mathrm{SiO}_{2}$ has been used in thermal chemical vapor deposition (CVD) processes for the production of tungsten silicides in a selflimiting tungsten deposition process. ${ }^{286}$ Comparable reactions were induced locally by irradiation of adsorbed $\mathrm{WF}_{6}$ layers on $\mathrm{SiO}_{2}$ on $\mathrm{Si}$. With $\mathrm{SiO}_{2}$ as substrate, above $50{ }^{\circ} \mathrm{C}$ etching occurs, whereas below this temperature deposition of $60 \%$ pure $\mathrm{W}$ was obtained. ${ }^{10}$ Further investigation in an adapted Auger electron microscope allowed Matsui et al. to determine that $\beta$-tungsten crystals were deposited. ${ }^{287}$ The electrical properties of such deposits revealed electrical resisitivities of $4 \times 10^{-3} \Omega \mathrm{cm}$ at room temperature corresponding to resistivity ratios (resistivity of deposit over pure metal) of about $100 .^{288,289}$ The importance of the absence of contamination on the samples prior to FEBID of $\mathrm{WF}_{6}$ has been demonstrated by an $\mathrm{O}_{2}$ plasma cleaning process carried out at $300{ }^{\circ} \mathrm{C}$ substrate temperature. ${ }^{290-292}$ Increasing the "deposition rate" was realized by adsorption of a layer of precursor prior to electron beam irradiation and therefore increased the efficiency of the deposition process. ${ }^{293,294}$ (b) Tin ( $\mathrm{Sn}$ ). Tin chloride $\left(\mathrm{SnCl}_{4}\right)$ condensed on silicon as a substrate was irradiated with different ions $\left(25 \mathrm{keV} \mathrm{H}_{2}^{+}\right.$, $50 \mathrm{keV} \mathrm{H}_{2}^{+}$, and $30 \mathrm{keV} \mathrm{Ar}^{+}$) and also $2 \mathrm{keV}$ electrons resulting in films that contained up to 52 at. \% Sn and 48 at. \% chlorine. Unfortunately, the relatively well conducting layers peeled off the substrate and, exposed to air, changed strongly due to hydrolysis of the chloride. ${ }^{295}$

(c) Titanium (Ti). $\mathrm{TiCl}_{4}$ was used by Kim and Somorjai to produce $\mathrm{TiCl}_{x}$ as active catalysts. After several low temperature condensation and thermal desorption processes, an increase in $\mathrm{Ti}$ in the gold substrate was detected. The latter results from desorption of chlorine from the surface. ${ }^{296}$ The same precursor also resulted in etching of PMMA resist and not in the wanted deposition of higher index of refraction $\mathrm{TiO}_{2}$ in the presence of residual water in an electron microscope. $^{297}$

(d) Indirect Si and $C$ from $\mathrm{AuCl}_{3}$. Using $\mathrm{AuCl}_{3}$ or $\mathrm{AlCl}_{3}$ as precursors in a scanning transmission electron microscope at $200 \mathrm{keV}$ on carbon and silicon substrates resulted in the deposition of either carbon or silicon pillars. ${ }^{246}$ The interpretation is an electron beam induced dissociation of the precursor resulting in the production of chlorine that reacts with substrate, forming volatile $\mathrm{SiCl}_{4}$ and $\mathrm{CCl}_{4}$. These compounds are then decomposed by FEBID to result in the observed $\mathrm{C}$ and $\mathrm{Si}$ deposits. As $\mathrm{AuCl}_{3}$ is not a very volatile compound, present in the gas phase as $\mathrm{Au}_{2} \mathrm{Cl}_{6}$ with an estimated vapor pressure of less than $1 \times 10^{-6}$ mbar, ${ }^{298}$ it might decompose to gold and $\mathrm{Cl}_{2}$ already in the precursor reservoir and only $\mathrm{Cl}_{2}$ leaves the nozzle. The observed final result would be identical.

(e) Hydrogen as coligand to halogeno precursors. Hydrogen as ligand is considered as a low contaminant ligand. For deposition of silicon in a transmission electron microscope at $120 \mathrm{kV}$ acceleration voltage and a spot size of $3 \mathrm{~nm}$, Ichihashi used $\mathrm{SiCl}_{2} \mathrm{H}_{2}$ as precursor. ${ }^{299}$ Dichlorosilane $\left(\mathrm{SiCl}_{2} \mathrm{H}_{2}\right)$ is an important intermediate compound in the purification of silicon and therefore commercially available with excellent purity. The deposition rate is given for a pressure of $10^{-5}$ Torr and the size of the obtained amorphous Si particles of $2 \mathrm{~nm}$ was presented. The study showed an increase of film growth with increasing precursor flow (measured indirectly with the pressure in the chamber). Additionally, it is mentioned that increasing the current density increases also the deposition rate (Table XIII). In a related work with the same precursor, but carried out in an Auger analysis system $\left(10 \mathrm{kV}, 0.14 \mathrm{~A} / \mathrm{cm}^{2}\right)$ revealed about $2 \% \mathrm{Cl}$ and $9 \% \mathrm{O}$ contamination; therefore 89 at.\% Si was measured. ${ }^{300}$

\section{Carbonyls}

Toxic, but often used precursors are metal carbonyls. The most prominent carbonyls used as precursors for FEBID or FIBID are $\mathrm{Ni}(\mathrm{CO})_{4}, \mathrm{Fe}(\mathrm{CO})_{5}, \mathrm{Fe}_{3}(\mathrm{CO})_{12}$, and $\mathrm{W}(\mathrm{CO})_{6}$. Carbonyl compounds are complexes with a central atom that is generally uncharged. The $\sigma$-donor- $\pi$-acceptor bond type between the uncharged ligands and the electrons in the $d$ orbitals of the central atom result in a configuration of the $\mathrm{CO}$ ligand with the carbon atom linked to the central metal atom 
TABLE XIII. FEB and FIB induced deposition with halogen based precursors. Enthalpies of evaporation and sublimation $\Delta H_{\text {vap/subl }}$, deposition conditions, deposit composition, and electrical resistivity are reported. Further symbols are $\varnothing$ (beam diameter), $P_{\text {res }}$ (residual vacuum pressure), $P_{\text {dep }}$ (pressure during deposition or estimated local pressure on sample surface), and $t_{\text {dep }}$ (deposition time).

\begin{tabular}{|c|c|c|c|c|}
\hline $\begin{array}{l}\text { Precursor } \\
\text { irradiation }\end{array}$ & $\begin{array}{l}\Delta H_{\mathrm{vap} / \mathrm{subl}} \\
\text { at RT } \\
(\mathrm{kJ} / \mathrm{mol})\end{array}$ & Conditions & $\begin{array}{l}\text { Composition } \\
\text { resistivity }\end{array}$ & Ref. \\
\hline $\begin{array}{l}\mathrm{WF}_{6} \\
20 \mathrm{keV} \text { electrons }\end{array}$ & $25.8^{\mathrm{a}}$ & $\begin{array}{l}\text { FEBID system: variable } \\
\text { currents, deposition at different temperatures. }\end{array}$ & $\cdots$ & 227 \\
\hline $\begin{array}{l}\mathrm{WF}_{6} \\
\text { electrons }\end{array}$ & $\cdots$ & $\begin{array}{l}\text { Auger system: } P_{\text {res }}: 10^{-10} \text { Torr } \\
10 \mathrm{keV}, 8 \times 10^{-4} \mathrm{~A} / \mathrm{cm}^{2} ; 2 \mathrm{C} / \mathrm{cm}^{2} \text {. } \\
\text { FEBID system: } 15 \mathrm{keV} ; 40 \mathrm{pA} \\
\text { deposition below } 50{ }^{\circ} \mathrm{C} \text {, etching } \\
\text { of } \mathrm{SiO}_{2} \text { above } 50^{\circ} \mathrm{C}\end{array}$ & $\begin{array}{l}56-66 \text { at. } \% \mathrm{~W}, \\
19 \text { at. } \% \mathrm{C} ; 14 \text { at. } \% \\
\mathrm{O} ; 1 \text { at. } \% \mathrm{~F}\end{array}$ & 10 \\
\hline $\begin{array}{l}\mathrm{WF}_{6} \\
\text { electrons }\end{array}$ & $\cdots$ & $\begin{array}{l}\text { Auger system: } \varnothing: 30 \mu \mathrm{m}, P_{\text {res }}: \\
2 \times 10^{-9} \text { Torr, } P_{\text {dep }}: 5 \times 10^{-7} \text { Torr, } \\
10 \mathrm{kV} ; 1 \mu \mathrm{A} ; 0.14 \mathrm{~A} / \mathrm{cm}^{2} ; \Delta T< \\
10 \mathrm{~K} \text {, substrate }(100) \mathrm{Si} \text {. } \\
\text { TEM system: } P_{\text {res }}: 3 \times 10^{-8} \text { Torr, } \\
P_{\text {dep }}: 0.1 \text { Torr, } 120 \mathrm{keV}, 100 \mathrm{~A} / \mathrm{cm}^{2} \text {, } \\
\text { substrate: Si particles }< \\
100 \text { nm diameter with } 1-3 \mathrm{~nm} \\
\text { SiO }_{2} \text { layer on } \mathrm{C} \text { grid (substrate } \\
\text { temperatue }<50^{\circ} \mathrm{C} \text { ). }\end{array}$ & $\begin{array}{l}85 \text { at. } \% \mathrm{~W}, 7.5 \text { at. } \% \\
\mathrm{~F} ; 7.5 \text { at. } \% \mathrm{O} \\
\beta-\mathrm{W} \text { cluster } \\
\text { coalescence } \\
\text { observed in situ }\end{array}$ & 287 and 299 \\
\hline $\begin{array}{l}\mathrm{WF}_{6} \\
0.5 \text { and } 2 \mathrm{keV} \\
\mathrm{Ar}^{+}\end{array}$ & $\cdots$ & $0.1 \mu \mathrm{A} / \mathrm{cm}^{2}$, substrate: $\mathrm{Si}_{3} \mathrm{~N}_{4}, \mathrm{Si}$ & $\begin{array}{l}\mathrm{W}: \mathrm{F}: \mathrm{C} \\
93.3: 4.4: 2.3 \\
15 \mu \Omega \mathrm{cm}\end{array}$ & 301 and 302 \\
\hline $\begin{array}{l}\mathrm{WCl}_{6} \\
\text { electrons }\end{array}$ & $\cdots$ & $\begin{array}{l}\text { Auger system: } 10 \mathrm{kV}, 8 \times 10^{-4} \mathrm{~A} / \mathrm{cm}^{2} ; 2 \mathrm{C} / \mathrm{cm}^{2} \\
\text { FEBID system: } 15 \mathrm{kV} ; 40 \mathrm{pA}\end{array}$ & $\begin{array}{l}58 \text { at. } \% \mathrm{~W} ; 16 \text { at. } \% \\
\mathrm{Cl} ; 8 \text { at. } \% \\
\mathrm{C} ; 18 \text { at. } \% \mathrm{O}\end{array}$ & 10 \\
\hline $\begin{array}{l}\mathrm{TiCl}_{4} \\
500 \text { eV electrons }\end{array}$ & $\cdots$ & $\begin{array}{l}100 \mathrm{~K} \text { substrate temperature, } \\
P_{\text {res }}: 9 \times 10^{-8} \text { Torr, } t_{\mathrm{dep}}: 8 \mathrm{~min} \text {, } \\
<10^{14} \text { electrons } / \mathrm{cm}^{2}\end{array}$ & $\begin{array}{l}\mathrm{TiCl}_{x}, x \text { not } \\
\text { specified }\end{array}$ & 296 \\
\hline $\begin{array}{l}\mathrm{SiH}_{2} \mathrm{Cl}_{2} \\
120 \mathrm{keV} \text { electrons }\end{array}$ & $22.2 \pm 0.7^{\mathrm{a}}$ & $\begin{array}{l}\text { Si particles }<100 \mathrm{~nm} \text { diameter } \\
\text { with nat oxide }(1-3 \mathrm{~nm}) \text { on } \mathrm{C} \\
\text { grid, } P_{\text {res }}: 5 \times 10^{-8} \text { Torr; pressure } \\
\text { measured at } 200 \mathrm{~mm} \text { from the } \\
\text { specimen, } 100 \mathrm{~A} / \mathrm{cm}^{2}\end{array}$ & Not speciefied & 299 \\
\hline $\begin{array}{l}\mathrm{SiH}_{2} \mathrm{Cl}_{2} \\
10 \mathrm{keV} \text { electrons }\end{array}$ & $\cdots$ & $\begin{array}{l}\varnothing: 30 \mu \mathrm{m}, \mathrm{Ge}(100) \text { substrate; } \\
P_{\text {res }}: 2 \times 10^{-9} \text { Torr, } P_{\text {dep }}: 0.1 \text { Torr, } \\
1 \mu \mathrm{A}, 0.14 \mathrm{~A} / \mathrm{cm}^{2}, \mathrm{MFC} \\
\text { precursor supply }\end{array}$ & $\begin{array}{l}9.1 \text { at. } \% \mathrm{O} ; 1.9 \text { at. } \% \\
\mathrm{Cl} ; 89 \text { at. } \% \\
\mathrm{Si}\end{array}$ & 300 \\
\hline $\begin{array}{l}\mathrm{CrO}_{2} \mathrm{Cl}_{2} \\
3 \mathrm{kV} \text { electrons }\end{array}$ & $\cdots$ & $\begin{array}{l}P_{\text {res }}: 2 \times 10^{-9} \text { Torr, } P_{\text {dep }}: 10^{-5} \text { Torr, } \\
t_{\text {dep }}: 5 \mathrm{~min}\end{array}$ & $\begin{array}{l}\mathrm{Cr}: \mathrm{O}: \mathrm{Cl} \\
1: 2.2: 1.1\end{array}$ & 303 \\
\hline
\end{tabular}

${ }^{\mathrm{a}}$ Reference 268.

due to the localization of the antibonding $\pi$ orbital into which the electron densities from the transition metal $d$ electrons are back donated on the $\mathrm{C}$ atom. The carbonyls are therefore complexes not needing any redox reaction in order to liberate atomic (uncharged) metals. On the other hand the special donor-acceptor bonds are relatively strong and a dissociation of a $\mathrm{CO}$ ligand by breaking the $\mathrm{Me}-\mathrm{C}$ bond from the metal atom is not easy. Furthermore, the dissociation of a carbonyl complex $\mathrm{Me}(\mathrm{CO})_{n}$ into the pure metal Me needs the separation from all the $\mathrm{CO}$ ligands. If such a complex is partially dissociated close to the surface of a metal, the metal carbonyl fragment will be attached to the metal surface and the CO ligands will have to be dissociated from the central metal atom. As the latter might be attached or even integrated into a metal lattice, the full dissociation of a carbonyl complex into pure metal corresponds to desorption of the $\mathrm{CO}$ ligands from the central metal bulk material. For this reason we will discuss briefly the electron beam induced desorption of molecules from metal surfaces. Accompanying this electron beam induced desorption is the low-energy electron induced dissociation of the $\mathrm{CO}$ on the metal surface that leads to carbon deposits and the liberation of $\mathrm{O}^{+} .{ }^{304}$ The desorption of CO from tungsten (211) was studied and revealed also the production of $\mathrm{O}^{+}$ions; ${ }^{305}$ the amount of neutral desorbed CO's, however, are not mentioned. In the case of molybdenum, the electron induced $\mathrm{CO}$ desorption is $10^{5}$ times more efficient than the dissociation and liberation of $\mathrm{O}^{+} .{ }^{306} \mathrm{Com}-$ parisons of the electron induced desorption of $\mathrm{CO}$ from Mo and $\mathrm{W}$ is not quantitative and not appropriate according to a later publication. ${ }^{115}$ On iridium (111) the dissociation is considered to present only $1 \%-2 \%$ of the electron induced desorption of $\mathrm{CO}^{307}$ In general with $\mathrm{CO}$ adsorbed on transition metal surfaces, several processes are going on with different yields. There is electron induced desorption of $\mathrm{CO}$, dissocia- 
tion into adsorbed carbon $\mathrm{C}_{\mathrm{ad}}$ and desorbed $\mathrm{O}^{+}$, and dissociation into adsorbed $\mathrm{C}_{\mathrm{ad}}$ and adsorbed $\mathrm{O}_{\mathrm{ad}}$. The latter might react with $\mathrm{CO}_{g}$ to produce $\mathrm{CO}_{2}$ and result in the $\mathrm{C}$ uptake on the surface of metals. Which path is most probable depends on several parameters such as $\mathrm{CO}$ coverage, partial pressure of $\mathrm{CO}$, transition metal, crystalline orientation of the metal, temperature, and irradiation conditions. We do not want to enter into details of these studies here, but want to show the variety of processes that might go on in FEB induced deposition experiments.

(a) Iron (Fe) from $\mathrm{Fe}(\mathrm{CO})_{5}$. Iron carbonyl $\left[\mathrm{Fe}(\mathrm{CO})_{5}\right]$ was used for FEBID by several groups resulting in different results. Early work resulted electron beam induced deposition on $\mathrm{Si}$ at room temperature and autocatalytic $\mathrm{Fe}$ film growth on $\mathrm{Si}$ heated to $250^{\circ} \mathrm{C}$. At an intermediate substrate temperature of $125^{\circ} \mathrm{C}$, selective autocatalytic growth of Fe on silicon occurred only in the electron beam irradiated region. ${ }^{308}$ The formation of iron subcarbonyls $\left[\mathrm{Fe}(\mathrm{CO})_{4}\right]$ on iron surfaces by transfer of carbonyl ligands from the adsorbed precursor to the substrate and the formation of subcarbonyl ions $\left[\mathrm{Fe}(\mathrm{CO})_{4}^{-} ; \mathrm{Fe}(\mathrm{CO})_{3}^{-}\right]$that had a high cross section for dissociative electron attachment reactions are mentioned and used for an explanation of the obtained results. ${ }^{309}$ The beam initiated and further thermal growth was studied also at higher temperatures, and the thermal deposition activation energy of $0.14 \mathrm{eV}$ could be obtained. ${ }^{310}$ For more details we refer to the original literature. Pure crystals of Fe could also be obtained by irradiation of a $10 \mathrm{~nm}$ thick $\mathrm{C}$ membrane around the irradiated region. ${ }^{311}$ The authors state that the process of $\mathrm{Fe}$ crystal growth differs from that observed by Kunz and Mayer. ${ }^{308,309}$ We think that catalytic decomposition of the iron carbonyl is responsible for the crystal growth and probably also beam heating effects. Another research team used iron carbonyl and decomposed it by doing FEBID in an UHV system, by writing at room temperature perpendicular into vacuum at $2-3 \mathrm{~nm} / \mathrm{s}$. It resulted in freestanding 50-30 nm diameter deposits. The deposits were amorphous and contained Fe and carbon. Pure $\alpha$-iron was obtained after annealing at $600{ }^{\circ} \mathrm{C}$ for $1 \mathrm{~h} .{ }^{312}$ Under slightly different depositon conditions with the same precursor, the composition of the deposit after $1 \mathrm{~h}$ annealing at $600{ }^{\circ} \mathrm{C}$ in vacuum is a mixture of $\alpha$-iron and several iron carbides. Carrying out the deposition with a $200 \mathrm{keV}$ electron beam on pure $7 \times 7 \mathrm{Si}$, heated to $850-900 \mathrm{~K}$, resulted in the growth of $\mathrm{FeSi}_{2} \cdot{ }^{313,314}$ Pure iron nanocrystal FEB deposition in an UHV system was obtained recently. ${ }^{315}$

Other higher nuclei number iron carbonyls $\left[\mathrm{Fe}_{3}(\mathrm{CO})_{12}\right]$ as precursor resulted in nanocomposite deposits, comparable to the simpler carbonyls. ${ }^{316}$ The approach of increasing the metal content by increasing the number of metal to metal bonds and decreasing the number of ligands were already developed in focused laser beam $^{317}$ or focused ion beam ${ }^{318,319}$ induced decomposition of metal organic compounds for multichip interconnects. It did improve the purity of the lines obtained in pyrolytic laser direct writing, but in FIB writing, reasonable electrical properties could only be obtained after reactive thermal annealing. In the case of pure gas phase processing, the increased number of nuclei generally increases the intermolecular van der Waals binding forces and therefore decreases the vapor pressure of the compound. Only for the reason of higher volatility small and simple mononuclear complex molecules even with large numbers of ligands (up to six) are generally taken for FEBID. The studies of UV irradiation of adsorbed $\mathrm{Fe}(\mathrm{CO})_{5}$ on Fe surfaces might help to understand the decomposition reactions. ${ }^{320}$

(b) Chromium ( $\mathrm{Cr}$ ) from $\mathrm{Cr}(\mathrm{CO})_{6}$. Chromium hexacarbonyl $\left[\mathrm{Cr}(\mathrm{CO})_{6}\right]$ was used by Kunz and Mayer for FEB induced deposition. ${ }^{310}$ They present the selective deposition of chromium at substrate temperatures higher than for the iron carbonyl deposition and determined the activation energy for thermal decomposition of $1.02 \mathrm{eV}$. The deposits carried out at substrate temperatures of 280 and $330{ }^{\circ} \mathrm{C}$ resulted in films with electrical resistivities of 141 and $79 \mu \Omega \mathrm{cm}$, respectively. The latter value is only six times higher than bulk chromium; the quality of the deposits was confirmed by the lack of any contamination measured by Auger spectroscopy. The comparison of the $\mathrm{Cr}$ deposition with the Fe deposition from the corresponding carbonyls was used for developing a generalized deposition model based on nucleation theory.

(c) Tungsten $(W)$ from $\mathrm{W}(\mathrm{CO})_{6}$. Tungsten hexacarbonyl $\left[\mathrm{W}(\mathrm{CO})_{6}\right]$ is used by numerous groups for FEB induced deposition. Very early publications reported compositions of typically 55 at. $\% \mathrm{~W}, 30$ at. $\% \mathrm{C}$, and 15 at. \% O. Some of the deposits contained even up to 75 at. $\%$ of W. The electrical resistivity with lines of $0.2 \Omega \mathrm{cm}$ was reported. ${ }^{11}$ More recently, the reported electrical conductivity of lines was improved (resistivities: $2.7 \times 10^{-2}$ and $4.5 \times 10^{-3} \Omega \mathrm{cm}$ ). ${ }^{64,65}$ Concerning the deposition mechanism, a large variation of the conditions was carried out and a two step deposition model was developed. ${ }^{233}$ After a first fragmentation of the precursor, resulting in an intermediate molecular fragment, a further fragmentation of the latter results in the final deposition product. The effect of electron dose and chemical composition was explained by this model. ${ }^{65}$ The $\mathrm{W}, \mathrm{O}$, and $\mathrm{C}$ containing deposits remain amorphous under almost all the deposition conditions; with high doses and low electron energies, the deposit density increases and a tendency of increased crystal size (still remaining very small) is observed. ${ }^{65}$ As discussed in more detail for the cobalt carbonyl, the dissociation mechanism might be even more complicated as the one described by Hoyle et al. ${ }^{233}$

(d) Cobalt (Co) from $\mathrm{Co}_{2}(\mathrm{CO})_{8}$. Cobalt carbonyl $\mathrm{Co}_{2}(\mathrm{CO})_{8}$ was applied for deposition of magnetic tips ${ }^{321,322}$ and electrically conductive lines for Hall sensors. ${ }^{323}$ The in depth study ${ }^{104}$ of the materials composition as a function of FEBID current revealed the variation of $18 \%-80 \%$ Co in the deposits explained by a thermal effect and the catalytic decomposition of the precursor on Co. The deposition of free space arches that are electrically conducting $(159 \mu \Omega \mathrm{cm})$ was achieved and the importance of the sufficiently short dwell time was explained by a low electrically conducting deposit at low electron densities. ${ }^{324}$ The variation in the deposit composition and properties with varying direct param- 
eters (electron current, acceleration voltage, and beam diameter) or indirect parameters (local temperature, local precursor coverage, presence, and amount of water vapor) is a puzzling story due to the multitude of processes going on during the deposition process. The adsorption of the precursor might already result in partial dissociation of the precursor or clustering to bigger $\mathrm{Co}-\mathrm{Co}$ bond containing units. Alternatively the complete integration into the metal surface might also happen, with the remaining adsorbed $\mathrm{CO}$ groups becoming surface adsorbed $\mathrm{CO}$ groups. The latter might dissociate into $\mathrm{C}$ and $\mathrm{O}$ adsorbates due to the increased local temperature from the metal bonds formed. Above $300 \mathrm{~K}$ dissociative adsorption with an activation energy for dissociation of $85 \pm 10 \mathrm{~kJ} / \mathrm{mol}$ competes with molecular adsorption on Co (1120). ${ }^{325}$ The molecular adsorbed CO might desorb from the surface by thermally induced desorption. On other planes of $\mathrm{Co}, \mathrm{CO}$ chemisorbs and dissociates under heating. The adsorbed oxygen diffuses into the $\mathrm{Co}$ and carbon forms a $\mathrm{Co}_{3} \mathrm{C}$ carbide. ${ }^{326}$ Adding electron irradiation increases the possibilities of the system to react. Electron induced desorption or electron induced dissociation is also reported in literature, or mentioned as side products of the analysis methods used for determining the thermal desorption processes. ${ }^{327}$ The authors present the dissociation and desorption of $\mathrm{CO}$ from polycrystalline cobalt. The dissociation takes place at $350 \mathrm{~K}$ and results in a 1:1 $\mathrm{C}$ to $\mathrm{O}$ coverage of the Co surface. From the dissociation rate constants, the dissociation activation energy of $71 \pm 8 \mathrm{~kJ} / \mathrm{mol}$ could be determined. The $\mathrm{CO}$ dissociation efficiency on polycrystalline cobalt differs from monocrystalline cobalt surfaces. Bridge measured the chemisorption of $\mathrm{CO}$ on $\mathrm{Co}(0001)$ and determined the electron $(80-100 \mathrm{eV})$ desorption cross section of $\mathrm{CO}$ on this surface to be $\geqslant 10^{-16} \mathrm{~cm}^{2}$. No dissociation was observed under the applied conditions. ${ }^{328}$ The dissociation probability seems to be a function of surface kinks and/or defects that will clearly appear during FEBID processing. Co undergoes rapid oxidation if exposed to $\mathrm{O}_{2}$. As water is one important residual gas in vacuum systems, measuring the effect of water on clean or $\mathrm{CO}$ covered Co surface would be of interest for further understanding the FEB induced deposition of Co.

\section{Pure phosphines and halogenophosphines}

Trifluorophosphine is an excellent $\sigma$-donor- $\pi$-acceptor ligand, resulting in complexes that are even more stable than the corresponding carbonyls. This is due to the strongest $\pi$-acceptor force of trifluorophosphine $\left(\mathrm{PF}_{3}\right)$ of all neutral ligands. ${ }^{329}$ It stabilizes therefore low charged transition metals in stable complexes. The stability of the complex would need a slightly higher substrate temperature for complete thermal dissociation than the corresponding carbonyls, as, for example, for nickel. For metals such as $\mathrm{Pt}$ and $\mathrm{Pd}$, the carbonyl is thermally not sufficiently stable at room temperature and therefore the corresponding trifluorophosphine complexes are dedicated precursors for FEB and FIB induced deposition. Furthermore the tendency of polymerization of phosphorus as compared to Carbon could be smaller and therefore the $\mathrm{PF}_{3}$ complexes looked very promising. (a) Pure trifluorophosphines. The first compound of this family is $\mathrm{Ni}\left(\mathrm{PF}_{3}\right)_{4}$ that has been used for FEB induced deposition recently. ${ }^{330}$ As compared to the carbonyls, the electron interaction cross section of the precursors should be higher. Unfortunately, the fragmentation of the trifluorophosphine ligands takes place under electron irradiation and nonnegligible amounts of $\mathrm{P}$ are codeposited with the metals. The $\mathrm{P}$ content in the deposits could be reduced from almost 60 at. $\%$ P to about 40 at. $\%$ by adding $\mathrm{O}_{2}$ in a 1:1 ratio. Also the temperature increase of the substrate decreased the P content in the deposits. However, both efforts did not result in pure Ni deposition. Another trifluorophosphine complex, tetrakistrifluorophosphine platinum $\mathrm{Pt}\left(\mathrm{PF}_{3}\right)_{4}$, has been used for deposition of Pt. This compound with a melting point of $-15^{\circ} \mathrm{C}$ and a boiling temperature at atmospheric pressure of $86{ }^{\circ} \mathrm{C}$ decomposes thermally at $90{ }^{\circ} \mathrm{C}$ according to textbooks of inorganic chemistry. Again codeposition of phosphorus was observed during FEB induced deposition ${ }^{331}$ probably due to the electron induced fragmentation of the adsorbed $\mathrm{PF}_{3}$ ligands that competes with the desorption rate of intact $\mathrm{PF}_{3}$ ligands from the deposits. Carrying out deposition under conditions where the desorption rate is higher than the fragmentation rate, higher purity can be obtained. A local temperature increase by several tens of degrees might explain the achieved carbon nanotube contacts that could be realized with this precursor. ${ }^{332}$

(b) Halogenotrifluorophosphines. The combination of the trifluorophosphine ligand with halogens $(\mathrm{Cl})$ opens another class of complexes. Herein the most interesting compound is the gold(I)trifluorophosphinochloride $\mathrm{AuClPF}_{3}$. The latter is a thermally fragile compound that undergoes decomposition reactions on the walls of metallic containers already at room temperature. The compound might evaporate as dimer or higher aggregate, but unfortunately there is not much information available about this compound. Applying $\mathrm{AuClPF}_{3}$ for FEB induced deposition resulted in one of the few deposits of pure metal by focused electron beam irradiation. ${ }^{333,334}$ This result can be compared to Kunz and Mayers' work on autocatalytic decomposition of $\mathrm{Fe}(\mathrm{CO})_{5}$ at $125^{\circ} \mathrm{C}$ and $\mathrm{Cr}(\mathrm{CO})_{6}$ at $330{ }^{\circ} \mathrm{C} .{ }^{310}$ In the case of the $\mathrm{AuClPF}_{3}$, the temperature window for selective deposition includes room temperature. Due to the very limited stability of the compound (it should be stored and always kept at temperatures below $-10{ }^{\circ} \mathrm{C}$ in order to avoid thermal decomposition), an industrial use of the compound does not appear easily feasible.

The rhodium and iridium chlorotrifluorophosphino complexes $\mathrm{RhCl}\left(\mathrm{PF}_{3}\right)_{2}$ exist as dimers: $\mathrm{Rh}_{2} \mathrm{Cl}_{2}\left(\mathrm{PF}_{3}\right)_{4}$ and $\mathrm{Ir}_{2} \mathrm{Cl}_{2}\left(\mathrm{PF}_{3}\right)_{4}$ were also successfully used for FEBID. ${ }^{335,336}$ The Rh compound and the Ir compound are both thermally much more stable than the $\mathrm{AuClPF}_{3}$ compound as shown for the $\mathrm{Rh}$ compound by the vapor pressure measurements. ${ }^{169}$ This might be the reason for the codeposition of amorphous $\mathrm{P}$ and other contaminants during FEBID growth (Table XIV). As already observed for the earlier mentioned precursors, the chemical composition of the $\mathrm{Rh}$ deposits did not vary strongly by varying the electron beam conditions. This includes also the water and partial oxygen content of the re- 
TABLE XIV. FEB and FIB induced deposition using carbonyl and trifluorophosphine precursors. Vapor pressures $P_{\text {vap}}$, deposit composition, and electrical resistivity are given. Values for the evaporation and sublimation enthalpies $\Delta H_{\text {vap/subl }}$ were collected from Refs. 169, 267, 268, and 337. Other symbols are $\varnothing$ (beam diameter), $P_{\text {res }}$ (residual vacuum pressure), $P_{\text {dep }}$ (pressure during deposition or estimated local pressure on sample surface), and $t_{\text {dep }}$ (deposition time).

\begin{tabular}{|c|c|c|c|c|c|}
\hline $\begin{array}{l}\text { Precursor } \\
\text { irradiation }\end{array}$ & $\begin{array}{l}P_{\text {vap }} \text { at } \\
\text { RT } \\
(\mathrm{mbar})\end{array}$ & $\begin{array}{l}\Delta H_{\mathrm{vap} / \mathrm{subl}} \\
\text { at RT } \\
(\mathrm{kJ} / \mathrm{mole})\end{array}$ & $\begin{array}{l}\text { Substrate } \\
\text { conditions }\end{array}$ & $\begin{array}{c}\text { Composition } \\
\text { resistivity }\end{array}$ & Ref. \\
\hline $\begin{array}{l}\mathrm{Fe}(\mathrm{CO})_{5} \\
0.5-3 \mathrm{keV} \\
\text { electrons }\end{array}$ & 47 & $38-40$ & $\begin{array}{l}\text { Si substrate, } 25-60 \mu \mathrm{A} / \mathrm{cm}^{2} \text {, } \\
\varnothing: 100 \mathrm{~nm}\end{array}$ & $\begin{array}{l}\mathrm{Fe} \text { crystals by } \\
\text { autocatalysis at } \\
125^{\circ} \mathrm{C}\end{array}$ & 308 and 338 \\
\hline $\begin{array}{l}\mathrm{Fe}(\mathrm{CO})_{5} \\
30 \mathrm{keV} \\
\text { electrons }\end{array}$ & $\cdots$ & $\cdots$ & $8 \times 10^{-10} \mathrm{~A}, \varnothing: 4 \mathrm{~nm}$ & Fe crystals & 312 \\
\hline $\begin{array}{l}\mathrm{Fe}(\mathrm{CO})_{5} \\
30 \mathrm{keV} \\
\text { electrons }\end{array}$ & $\cdots$ & & $\begin{array}{l}10 \mathrm{~nm} \text { thin } \mathrm{C} \text { grid, } P_{\text {res }}: \\
2 \times 10^{-6} \mathrm{~Pa} \text {; UHV-SEM, } \\
8 \times 10^{-10} \mathrm{~A} ; \varnothing: 4 \mathrm{~nm}\end{array}$ & Fe crystals & 339 and 340 \\
\hline $\begin{array}{l}\mathrm{Fe}(\mathrm{CO})_{5} \\
300 \mathrm{keV} \\
\text { electrons }\end{array}$ & $\cdots$ & & $\begin{array}{l}\mathrm{Si}(111) ; 850-900 \mathrm{~K}, \\
P_{\text {res }}: 5 \times 10^{-8} \mathrm{~Pa} \text { UHV- } \\
\mathrm{TEM}, 5 \times 10^{-3}-5 \times 10^{-4} \mathrm{~A} / \mathrm{cm}^{2}\end{array}$ & $\mathrm{FeSi}_{2}$ crystals & 313 and 341 \\
\hline $\begin{array}{l}\mathrm{Fe}(\mathrm{CO})_{5} \\
15 \mathrm{keV} \\
\text { electrons }\end{array}$ & & & $\mathrm{Si}(001), \mathrm{UHV} 10^{-10} \mathrm{mbar}$ & Fe crystals & 315 \\
\hline $\begin{array}{l}\mathrm{Cr}(\mathrm{CO})_{6} \\
\text { electrons }\end{array}$ & $0.18^{\mathrm{b}}$ & $\begin{array}{l}70-62.5 \\
(324 \mathrm{~K})\end{array}$ & $\begin{array}{c}p_{\text {res }}: 8 \times 10^{-7} \mathrm{Torr} \\
\mathrm{Si}, 25^{\circ} \mathrm{C}-400^{\circ} \mathrm{C} \\
10-100 \mathrm{microA} / \mathrm{cm}^{2}\end{array}$ & $10-10^{8} \mu \Omega \mathrm{cm}$ & 310 and 342 \\
\hline $\begin{array}{l}\mathrm{Mo}(\mathrm{CO})_{6} \\
\text { electrons }\end{array}$ & $0.15^{\mathrm{a}, \mathrm{b}}$ & 77.7 & $\cdots$ & $\begin{array}{l}2 \times 10^{-3} \Omega \mathrm{cm} \\
10 \text { at. } \% \mathrm{Mo}\end{array}$ & 343 \\
\hline $\begin{array}{l}\mathrm{W}(\mathrm{CO})_{6} \\
3 \mathrm{keV} \\
\text { electrons }\end{array}$ & $0.023 / 23{ }^{\circ} \mathrm{C}$ & 77.7 & $\begin{array}{l}P_{\text {res }}: 1.2 \times 10^{-6} \mathrm{mbar} \\
P_{\text {dep }}: 3.5 \times 10^{-6} \mathrm{mbar}, 150 \\
\text { and } 590 \mathrm{pA} \text {, dwell } \\
\text { time } 30-300 \mathrm{~ms}\end{array}$ & $\cdots$ & 344 \\
\hline $\begin{array}{l}\mathrm{W}(\mathrm{CO})_{6} \\
25 \mathrm{keV} \\
\text { electrons }\end{array}$ & $\cdots$ & $\cdots$ & $\begin{array}{l}\mathrm{Si}_{3} \mathrm{~N}_{4} \text { membrane at }-3{ }^{\circ} \mathrm{C} \\
10^{8} \mathrm{~A}, \varnothing: 0.15 \mu \mathrm{m}, \\
P_{\text {dep }}: 2 \text { mTorr }\end{array}$ & $\begin{array}{l}55 \text { at. } \% \mathrm{~W}, 30 \text { at. } \% \mathrm{C}, \\
15 \text { at. } \% \mathrm{O} ; \\
75 \text { at. } \% \mathrm{~W} \text { (best), } \\
0.2 \Omega \mathrm{cm}\end{array}$ & 11 \\
\hline $\begin{array}{l}\mathrm{W}(\mathrm{CO})_{6} \\
5 \mathrm{keV} \\
\text { electrons }\end{array}$ & $\cdots$ & $\cdots$ & $\begin{array}{l}\text { GaAs at } 22^{\circ} \mathrm{C}, \\
P_{\text {res }}: 3 \times 10^{-6} \text { Torr; } \\
P_{\text {dep }}: 9 \times 10^{-6} \text { Torr; } \\
0.94-1 \mathrm{nA}, \varnothing: 400 \mathrm{~nm}, \\
\text { loop time varied, } \\
9 \times 10^{15} \text { molecules } / \mathrm{cm}^{2} \mathrm{~s}\end{array}$ & $3 \times 10^{-3} \Omega \mathrm{cm}$ & 233 \\
\hline $\begin{array}{l}\mathrm{W}(\mathrm{CO})_{6} \\
0.06-20 \mathrm{keV} \\
\text { electrons }\end{array}$ & $\cdots$ & $\cdots$ & $\begin{array}{l}\text { GaAs at } 22^{\circ} \mathrm{C} \\
P_{\text {res }}: 3 \times 10^{-6} \text { Torr; } \\
P_{\text {dep }}: 9 \times 10^{-6} \text { Torr; } \\
4 \mathrm{nA}, \varnothing: 0.3-0.5 \mu \mathrm{m}, \\
\text { loop times } 6 \text { and } 60 \mathrm{~s} \\
1.4 \times 10^{16} \text { molecules } / \mathrm{cm}^{2} \mathrm{~s}\end{array}$ & $6 \times 10^{-4} \Omega \mathrm{cm}$ & 64 and 65 \\
\hline $\begin{array}{l}\mathrm{W}(\mathrm{CO})_{6} \\
25 \mathrm{keV} \mathrm{Ga}^{+}\end{array}$ & $\cdots$ & $\cdots$ & Total yield: 2 atoms/ion & $\begin{array}{l}\mathrm{W}: \mathrm{C}: \mathrm{Ga}: \mathrm{O} \\
75: 10: 10: 5 \\
150-225 \mu \Omega \mathrm{cm}\end{array}$ & 345 \\
\hline $\begin{array}{l}\mathrm{W}(\mathrm{CO})_{6} \\
30 \mathrm{keV} \mathrm{Ga}^{+}\end{array}$ & $\cdots$ & $\cdots$ & $\cdots$ & $\begin{array}{l}\text { W:C:Ga } \\
51: 37: 12 \\
200 \mu \Omega \mathrm{cm}, \\
\text { superconducting } \\
\text { for } T<5.5 \mathrm{~K}\end{array}$ & 346 \\
\hline $\begin{array}{l}\mathrm{Co}_{2}(\mathrm{CO})_{8} \\
25 \mathrm{keV} \\
\text { electrons }\end{array}$ & $0.4^{\mathrm{b}}$ & $\begin{array}{l}84 \\
65\end{array}$ & $\begin{array}{l}\mathrm{SiO}_{2} \text { on } \mathrm{Si} \text { at } 22{ }^{\circ} \mathrm{C} \\
P_{\text {res }}: 1 \times 10^{-6} \mathrm{Torr}, \\
0.5 \mathrm{nA}, \varnothing: 200 \mathrm{~nm}\end{array}$ & $7 \times 10^{-6} \Omega \mathrm{cm}$ & 323 \\
\hline $\begin{array}{l}\mathrm{Co}_{2}(\mathrm{CO})_{8} \\
25 \mathrm{keV} \\
\text { electrons }\end{array}$ & $\cdots$ & $\cdots$ & $\begin{array}{l}25 \mathrm{keV} ; 0.5 \mathrm{nA} \\
\varnothing: 200 \mathrm{~nm} \\
30 \mathrm{~nm} / \mathrm{s}\end{array}$ & $\begin{array}{l}34 \text { at. } \% \mathrm{Co} \\
51 \text { at. } \% \mathrm{C} \\
14 \text { at. } \% \mathrm{O} \text {, } \\
2-5 \mathrm{~nm} \text { crystals of cubic Co }\end{array}$ & 322 \\
\hline $\begin{array}{l}\mathrm{Co}_{2}(\mathrm{CO})_{8} \\
25 \mathrm{keV} \\
\text { electrons }\end{array}$ & $\cdots$ & $\cdots$ & $\begin{array}{l}25 \mathrm{keV}, 2-5 \mathrm{nA}, \\
25 \mathrm{keV} ; \\
20 \mathrm{pA}-3 \mu \mathrm{A} ; 2-45 \mathrm{~nm} / \mathrm{s}\end{array}$ & $12-80$ at. $\% \mathrm{Co}$ & 104 and 204 \\
\hline
\end{tabular}


TABLE XIV. (Continued.)

\begin{tabular}{|c|c|c|c|c|c|}
\hline $\begin{array}{l}\text { Precursor } \\
\text { irradiation }\end{array}$ & $\begin{array}{l}P_{\text {vap }} \text { at } \\
\text { RT } \\
(\mathrm{mbar})\end{array}$ & $\begin{array}{l}\Delta H_{\mathrm{vap} / \mathrm{subl}} \\
\text { at RT } \\
(\mathrm{kJ} / \mathrm{mole})\end{array}$ & $\begin{array}{l}\text { Substrate } \\
\text { conditions }\end{array}$ & $\begin{array}{c}\text { Composition } \\
\text { resistivity }\end{array}$ & Ref. \\
\hline $\begin{array}{l}\mathrm{Co}_{2}(\mathrm{CO})_{8} \\
15-30 \mathrm{keV} \\
\text { electrons }\end{array}$ & $\cdots$ & $\begin{array}{l}\text { Heated } \\
\text { precursor } \\
\text { reservoir }\end{array}$ & $\begin{array}{l}P_{\text {res }}: 10^{-6} \mathrm{mbar}, \\
P_{\text {dep }}:(3-6) \times 10^{-5} \mathrm{mbar} ; \\
180 \mathrm{pA}-13.5 \mathrm{nA}, t_{\mathrm{dep}}: 3-180 \mathrm{~s}\end{array}$ & $\begin{array}{l}30-50 \text { at. } \% \text { Co } \\
45 \mu \Omega \mathrm{cm} ; \\
\text { bridges: } 159 \mu \Omega \mathrm{cm}\end{array}$ & 324 \\
\hline $\begin{array}{l}\mathrm{Co}(\mathrm{CO})_{3}(\mathrm{NO}) \\
15-30 \mathrm{keV} \\
\text { electrons }\end{array}$ & $30 / 45^{\circ} \mathrm{C}$ & $\begin{array}{l}\text { Heated } \\
\text { precursor } \\
\text { reservoir }\end{array}$ & $\begin{array}{l}P_{\text {res }}: 10^{-6} \mathrm{mbar}, \\
P_{\text {dep }}:(3-6) \times 10^{-5} \mathrm{mbar} ; \\
180 \mathrm{pA}-13.5 \mathrm{nA}, t_{\mathrm{dep}}: 3-180 \mathrm{~s}\end{array}$ & $\begin{array}{l}\text { Pure catalytic } \\
\text { Co deposits }\end{array}$ & 324 \\
\hline $\begin{array}{l}\mathrm{Os}_{3}(\mathrm{CO})_{12} \\
40 \mathrm{keV} \\
\text { electrons }\end{array}$ & $7 \times 10^{-5 b}$ & $104.6 \pm 20$ & $\begin{array}{l}\mathrm{Si}(100) \text { at }-40^{\circ} \mathrm{C} \\
P_{\text {dep }}: 5 \times 10^{-6} \mathrm{mbar} \\
0.8-15 \mathrm{~mA} / \mathrm{cm}^{2}\end{array}$ & $\begin{array}{l}\text { Up to } 20 \mathrm{~nm} \\
\text { sized grains }\end{array}$ & 187 \\
\hline $\begin{array}{l}\mathrm{Ru}_{3}(\mathrm{CO})_{12} \\
40 \mathrm{keV} \\
\text { electrons }\end{array}$ & $\cdots$ & $\cdots$ & $\begin{array}{l}\mathrm{Si}(100)-40^{\circ} \mathrm{C} \\
P_{\mathrm{dep}}: 5 \times 10^{-6} \mathrm{mbar} \\
0.8-15 \mathrm{~mA} / \mathrm{cm}^{2}\end{array}$ & $\begin{array}{l}\text { Up to } 20 \mathrm{~nm} \\
\text { sized grains }\end{array}$ & 187 \\
\hline $\begin{array}{l}\mathrm{Fe}_{3}(\mathrm{CO})_{12} \\
15 \text { and } 20 \mathrm{keV} \\
\text { electrons }\end{array}$ & $1 \times 10^{-3}$ & $\cdots$ & $\begin{array}{l}5-200 \mathrm{nA} \\
\varnothing: 0.2-0.5 \mu \mathrm{m}\end{array}$ & $4 \times 10^{-2}-1 \times 10^{4} \Omega \mathrm{cm}$ & 316 \\
\hline $\begin{array}{l}\mathrm{Rh}_{2} \mathrm{Cl}_{2}(\mathrm{CO})_{4} \\
25 \mathrm{keV} \\
\text { electrons }\end{array}$ & $0.0025^{\mathrm{c}}$ & 84 & $0.5-5 \mathrm{nA}$ & $\begin{array}{l}56 \text { at. } \% \mathrm{Rh}, 34 \text { at. } \% \mathrm{C}, \\
5 \text { at. } \% \mathrm{Cl} ; \\
2 \text { at. } \% \mathrm{O}, 3 \text { at. } \% \mathrm{~N}\end{array}$ & 347 \\
\hline $\begin{array}{l}\mathrm{Ni}\left(\mathrm{PF}_{3}\right)_{4} \\
10-25 \mathrm{keV} \\
\text { electrons }\end{array}$ & $\begin{array}{l}160 \\
147^{\mathrm{d}}\end{array}$ & $\cdots$ & $\begin{array}{l}2-250 \mathrm{nA}, \varnothing: 0.1-5 \mu \mathrm{m}, \\
P_{\text {res }}: 10^{-6} \mathrm{mbar}, \\
P_{\mathrm{dep}}: 3 \times 10^{-5} \mathrm{mbar}\end{array}$ & $\begin{array}{l}36 \text { at. } \% \mathrm{Ni}, 22 \text { at. } \% \mathrm{O}, \\
14 \text { at. } \% \mathrm{C}, \\
17 \text { at. } \% \mathrm{P}, 11 \text { at. } \% \mathrm{~F}, \\
\rho / \rho_{0}=140\end{array}$ & 330 \\
\hline $\begin{array}{l}\mathrm{Pt}\left(\mathrm{PF}_{3}\right)_{4} \\
10 \mathrm{keV} \\
\text { electrons }\end{array}$ & $\cdots$ & 15 Torr $/ 0^{\circ} \mathrm{C}$ & $\begin{array}{l}\text { FEI } 620 \text { dual beam } \\
0.6-46 \mathrm{nA}\end{array}$ & $\begin{array}{l}81 \% \mathrm{Pt}, 17 \% \mathrm{P} \\
1 \% \mathrm{~F} \\
\rho / \rho_{0} \sim 3\end{array}$ & 332 \\
\hline $\begin{array}{l}\mathrm{AuClPF}_{3} \\
25 \mathrm{keV} \\
\text { electrons }\end{array}$ & $\cdots$ & $\cdots$ & $100 \mathrm{pA}-1 \mathrm{nA}$ & $\begin{array}{l}>95 \text { at. } \% \mathrm{Au} \\
43 \mu \Omega \mathrm{cm}\end{array}$ & 333 and 349 \\
\hline $\begin{array}{l}\mathrm{AlH}_{3}-\mathrm{N}\left(\mathrm{CH}_{3}\right)_{3} \\
20 \mathrm{keVGa}^{+}\end{array}$ & $\cdots$ & $\cdots$ & Total yield: 5 atoms/ion & $\begin{array}{l}\mathrm{Al}: \mathrm{Ga}: \mathrm{C}: \mathrm{N} \\
29: 12: 31: 28 \\
900 \mu \Omega \mathrm{cm}\end{array}$ & 350 \\
\hline $\begin{array}{l}\mathrm{Rh}_{2} \mathrm{Cl}_{2}\left(\mathrm{PF}_{3}\right)_{4} \\
25 \mathrm{keV} \\
\text { electrons }\end{array}$ & $0.075^{\mathrm{a}}$ & 90.8 & $\begin{array}{l}1-5 \mathrm{nA} \\
2 \times 10^{17} \text { molecules } / \mathrm{cm}^{2} \mathrm{~s} \\
20 \mathrm{~nm} / \mathrm{s}\end{array}$ & $\begin{array}{l}\mathrm{Rh}_{0.66} \mathrm{P}_{0.33}, \\
60 \text { at. } \% \mathrm{Rh}, 18 \text { at. } \% \mathrm{P}, \\
7 \text { at. } \% \mathrm{Cl}, \\
8 \text { at. } \% \mathrm{O}, 7 \text { at. } \% \mathrm{~N}\end{array}$ & 258 and 347 \\
\hline $\begin{array}{l}\mathrm{Ir}_{2} \mathrm{Cl}_{2}\left(\mathrm{PF}_{3}\right)_{4} \\
25 \mathrm{keV} \\
\text { electrons }\end{array}$ & $\cdots$ & $\cdots$ & $\begin{array}{l}0.5-5 \mathrm{nA}, \\
7 \times 10^{16} \text { molecules } / \mathrm{cm}^{2} \mathrm{~s}\end{array}$ & $\begin{array}{l}\mathrm{Ir}: \mathrm{Cl}: \mathrm{P} \\
1: 1: 1\end{array}$ & 336 \\
\hline
\end{tabular}

${ }^{\mathrm{a}}$ Reference 169 .

${ }^{\mathrm{b}}$ Reference 170.

${ }^{\mathrm{c}}$ Reference 337.

${ }^{\mathrm{d}}$ Reference 348.

sidual gas. The deposits contained 60 at. \% metal in an amorphous matrix that contained mainly $\mathrm{P}$ but also some $\mathrm{C}, \mathrm{O}$, and $\mathrm{N}^{335}$

\section{Organometallics}

Organometallic molecular compounds are in literature also sometimes called metal organic compounds or metalloorganic compounds without a clear definition of the words. Here we use organometallics for compounds with carbon to metal bonds. According to this definition, metal carbonyls would enter also into this group, but as they form a large group of precursors used in FEBID, we treated them independently above.

The organometallic compounds differ in their bond structure depending on the difference in electronegativity and type of bonding. Simple $\sigma$-type bonds are involved in light main group metals and transition metals with alkyl groups, delocalized bonds involving double bonds, triple bonds, and aromatic systems are generally weaker ligand to metal bonds. As an example the bisbenzene chromium $\left(\mathrm{C}_{6} \mathrm{H}_{6}\right)_{2} \mathrm{Cr}$ is considered to be unstable at room temperature. ${ }^{351}$ More complicated is the bond cleavage of $\mathrm{Si}-\mathrm{C}$ in alkylsilanes or $\mathrm{Al}-\mathrm{C}$ in alkylalanes.

(a) Metal alkyls $\left[\mathrm{Si}\left(\mathrm{CH}_{3}\right)_{4}, \mathrm{Al}\left(\mathrm{CH}_{3}\right)_{3}, \mathrm{Sn}\left(\mathrm{CH}_{3}\right)_{4}\right]$. FEBID of simple metal alkyls result generally in metal and carbon containing materials. The irradiation of tetramethylsilane (TMS) in a SEM results in the deposition of partially oxidized amorphous silicon and carbon rich deposits. ${ }^{352,353}$ With increasing precursor flow, the oxygen uptake in the deposit decreases. This was explained by the decreasing ratio of re- 
sidual water vapor as compared to the precursor molecules. The water vapor clearly determined the oxygen uptake during the FEB induced deposition. Intentional addition of molecular oxygen resulted in the deposition of pure $\mathrm{SiO}_{2}$ and will be discussed in the corresponding section below. Trimethylaluminum was irradiated with focused electrons and lines were obtained. The EDX measurements revealed Al and C; no in-depth analysis of the deposits was carried out. ${ }^{354}$

About $2 \mu \mathrm{m}$ thick films of tetramethyltin $\left[\mathrm{Sn}\left(\mathrm{CH}_{3}\right)_{4}\right]$ condensed at $120 \mathrm{~K}$ on silicon as substrate were irradiated with different ions and also $2 \mathrm{keV}$ electrons resulting in films that contained unspecified amounts of Sn. The remaining C (and partially also hydrogen) in the deposit does not desorb and therefore explained the low measured electrical conductivity. Hydrogen is lost mainly due to fragmentation of the methyl ligands and not due to $\mathrm{Sn}-\mathrm{C}$ bond braking. The electron dissociation cross section of tetramethyltin at $2 \mathrm{keV}$ is between 1.8 and $3.4 \AA^{2}$ and is very close to the electron dissociation cross section of methane in the gas phase of $2.9 \AA^{2}$ for electrons of $0-600 \mathrm{eV} .{ }^{355}$ Higher electrically conducting films $\left(\rho / \rho_{0}=50\right)$ could be obtained by irradiation with $\mathrm{Ar}^{+}$ions, explained by a selective sputtering process.

(b) Sandwich aromatic or pseudoaromatic complexes. The stability of the aromatic sandwich complex bisbenzene chromium(0) $\left(\mathrm{C}_{6} \mathrm{H}_{6}\right)_{2} \mathrm{Cr}$, first synthesized in $1955,{ }^{356}$ as reported in literature varies from room temperature ${ }^{351}$ to stable at $100{ }^{\circ} \mathrm{C}$ for evaporation. ${ }^{357}$ The bisbenzene chromium was tested for FEB induced deposition, ${ }^{10}$ and the cyclopentadienyl complexes ferrocene, nickelocene, cobaltocene, $\left(\mathrm{C}_{5} \mathrm{H}_{5}\right)_{2} \mathrm{Met}$ with $\mathrm{Met}=\mathrm{Fe}, \mathrm{Ni}, \mathrm{Co}$, respectively, were proposed for FEB induced deposition of magnetic deposits in a patent. ${ }^{358}$ Ferrocene was furthermore used for deposits obtained by STM-CVD, but no chemical composition is stated. $^{359}$

All FEBID experiments from metal organic compounds reported in literature with quantitative information about the chemical composition fall into a range between 10 and 25 at. \% of metal content in the deposits. Relatively large amounts of carbon are generally codeposited (Table XV).

\section{Acetylacetonates}

According to a review article about the oxygen-metal bond energetics in $\beta$-diketonates, ${ }^{369}$ the other name for acetylacetonates, these compounds are known already since 1887. They are an important class of volatile metal atom containing compounds and therefore widely used as precursors in CVD processes. The $\beta$-diketonate ligands are very stable ligands, forming a six membered flat aromatic ring system including the central (metal) atom as one of the hexagon edges. The charge is delocalized between all the six atoms and the rigid structure protects the central atom efficiently against chemical reactions. Variation in the $\beta$-diketonates from the simple acetyl acetonate by replacing the hydrogen atoms of one (both) methyl group(s) by three (six) fluorine atoms results in the more volatile trifluoroacetylacetonate (hexafluoroacetylacetonate) compounds.
This substitution appears to be more efficient for increasing volatility than the increase of the asymmetry of the ligands or the increase of steric hindrance by replacing the methyl group by bulkier tertiary butyl groups. The acetylacetonate groups are relatively stable ligands that do have to be dissociated, fragmented, or chemically reacted, for example, by oxidation or reduction in order to get rid of them from a metal surface. The latter takes place generally at temperatures above $200{ }^{\circ} \mathrm{C}$. The differences in thermal decomposition of copper acetylacetonates differ for $\mathrm{Cu}(\mathrm{I})$ and $\mathrm{Cu}(\mathrm{II})$ acetylacetonates, and the photolytical decomposition differs from the thermal one according to the literature. ${ }^{168}$ The disproportionation reaction of the $\mathrm{Cu}(\mathrm{I})$ compounds after having lost the weakly attached uncharged ligands results in clean copper deposits at sufficiently high temperature of the substrate $\left(>120^{\circ} \mathrm{C}\right)$ and desorption of the bisacetylacetonato$\mathrm{Cu}$ (II) complex. For decomposition of the $\mathrm{Cu}$ (II) compounds, substrate temperatures of $200{ }^{\circ} \mathrm{C}$ or higher and additional reactive gases are needed for obtaining pure copper deposits. ${ }^{168}$ The group of dimethylgold acetylacetonato compounds decompose thermally under the formation of ethane and a fragmentation of the acetylacetonato ligands at temperatures above $200{ }^{\circ} \mathrm{C} .{ }^{168}$ The ethane is produced by forming a $\mathrm{C}-\mathrm{C}$ bond between the two carbon atoms of the methyl groups (Table XVI). A mechanism explaining the carbon insertion into the deposits from the decomposition of the acetylacetonato ligand on a copper surface is presented in literature. ${ }^{168}$

\section{Alkoxides, nitrates, and amides}

The most popular precursors for oxide deposition are the alkoxides. Hydrolysis results in alcohols, which are much less corrosive as compared to the hydrolysis product of halides. Alkoxides contain organic ligands with oxygen bonds to the central atom. Well known are the silicon alkoxide tetraethoxysilane (TEOS) also called tetra-ethyl-ortho-silicate and the corresponding methyl analog tetramethoxysilane (TMOS). Both contain four alkoxy ligands. For titanium, the highest volatile alkoxide is the titanium tetraisopropoxide (TTIP); for tantalum the tantalum pentaethoxide $\left[\mathrm{Ta}(\mathrm{OEt})_{5}\right]$ is commonly used for tantalum oxide deposition. Alkoxides are water sensitive and undergo hydrolysis reactions at room temperature, liberating the corresponding alcohols and forming hydroxyl groups attached to the central atom. These undergo wanted or unwanted condensation reactions and result in oligomers in a three dimensional network of metal oxide. This is a wanted reaction in the case of sol-gel processes. For FEBID and FIBID the presence of water either in the precursor reservoir or in the vacuum chamber is strictly to be avoided if working with alkoxides due to the above mentioned chemical reactions. They result in very low volatile and sticky oligomers that are eternally contaminating vacuum systems.

The depositions of Si containing materials from precursors that contain already oxygen, nitrogen, or carbon bonds to $\mathrm{Si}$ are very unlikely to produce pure silicon. Si could be produced from halogen and/or hydrogen containing silanes 
TABLE XV. FEB and FIB induced deposition using organometallic precursors. Vapor pressures $P_{\text {vap }}$, deposition conditions, deposit composition, and electrical resistivity are given. Values for the evaporation and sublimation enthalpies $\Delta H_{\mathrm{vap} / \mathrm{subl}}$ were collected from Refs. 268 and 360 . Other symbols are $\varnothing$ (beam diameter), $P_{\text {res }}$ (residual vacuum pressure), $P_{\text {dep }}$ (pressure during deposition or estimated local pressure on sample surface), and $t_{\text {dep }}($ deposition time).

\begin{tabular}{|c|c|c|c|c|c|c|}
\hline Precursor & Name & $\begin{array}{l}P_{\text {vap }} \text { at } \\
\text { RT } \\
\text { (mbar) }\end{array}$ & $\begin{array}{l}\Delta H_{\mathrm{vap} / \mathrm{subl}} \\
\text { at } \mathrm{RT} \\
(\mathrm{kJ} / \mathrm{mol})\end{array}$ & Conditions & $\begin{array}{l}\text { Composition } \\
\text { (at \%) } \\
\text { resistivity }\end{array}$ & Ref. \\
\hline $\begin{array}{l}\mathrm{Si}\left(\mathrm{CH}_{3}\right)_{4} \\
10 \mathrm{keV} \\
\text { electrons }\end{array}$ & $\begin{array}{l}\text { Tetramethylsilane } \\
\text { (TMS) }\end{array}$ & $804 / 20{ }^{\circ} \mathrm{C}$ & $\cdots$ & 125 pA, Diam: 5.8 micrometers & $\mathrm{SiO}_{1.8} \mathrm{C}_{3.7}$ & 352 and 353 \\
\hline $\begin{array}{l}\mathrm{Al}\left(\mathrm{CH}_{3}\right)_{3} \\
6 \mathrm{keV} \\
\text { electrons }\end{array}$ & Trimethylalane & $\cdots$ & $\begin{array}{l}39.8 \\
(351 \mathrm{~K})\end{array}$ & $\begin{array}{l}\text { GaAs, } \varnothing: 8 \mathrm{~nm}, P_{\text {res }}: \\
5 \times 10^{-8} \text { Torr, } \\
P_{\text {dep }}: 1 \times 10^{-6} \text { Torr, } 10^{-11} \mathrm{~A}\end{array}$ & $\begin{array}{l}\text { Al with some } \\
\mathrm{C} \text {; not } \\
\text { quantified }\end{array}$ & 354 \\
\hline $\begin{array}{l}\mathrm{Sn}\left(\mathrm{CH}_{3}\right)_{4} \\
2 \mathrm{keV} \\
\text { electrons }\end{array}$ & $\begin{array}{l}\text { Tetramethyl } \\
\text { tin }\end{array}$ & 132 & $30-33$ & $\begin{array}{l}\text { Precursor } \\
\text { condensation at } 120 \mathrm{~K}, \\
\text { irradiation } 3.2 \times 3.2 \mathrm{~mm}^{2}\end{array}$ & $\cdots$ & 355 \\
\hline $\begin{array}{l}\mathrm{Cr}\left(\mathrm{C}_{6} \mathrm{H}_{6}\right)_{2} \\
\text { electrons }\end{array}$ & $\begin{array}{l}\text { Bisbenzene } \\
\text { chromium }\end{array}$ & $\cdots$ & $\begin{array}{l}78-90 ; 49.5 \\
(485 \mathrm{~K})\end{array}$ & $\begin{array}{l}\text { Auger system: } 10 \mathrm{keV} \text {, } \\
8 \times 10^{-4} \mathrm{~A} / \mathrm{cm}^{2}, 2 \mathrm{C} / \mathrm{cm}^{2} \text {, } \\
\text { FEBID system: } 15 \mathrm{kV} \text {; } \\
40 \mathrm{pA}\end{array}$ & $\begin{array}{l}16 \text { at. } \% \mathrm{Cr}, 62 \text { at. } \% \\
\mathrm{C} ; 22 \text { at. } \% \mathrm{O}\end{array}$ & 10 \\
\hline $\mathrm{Fe}\left(\mathrm{C}_{5} \mathrm{H}_{5}\right)_{2}$ & Ferrocene & $\begin{array}{l}4.1 \times 10^{-3} \\
1.2 \times 10^{-2 \mathrm{a}}\end{array}$ & $\begin{array}{l}47.3 \\
(471 \mathrm{~K}) ; 73 \\
71\end{array}$ & No details & No details & 358 \\
\hline $\mathrm{Ni}\left(\mathrm{C}_{5} \mathrm{H}_{5}\right)_{2}$ & Nickelocene & $\begin{array}{l}8 \text { Torr at } \\
110^{\circ} \mathrm{C}\end{array}$ & & No details & No details & 358 \\
\hline $\mathrm{Co}\left(\mathrm{C}_{5} \mathrm{H}_{5}\right)_{2}$ & Cobaltocene & & & No details & No details & 358 \\
\hline $\begin{array}{l}\mathrm{Ni}(\mathrm{MeCp})_{2} \\
10-25 \mathrm{keV} \\
\text { electrons }\end{array}$ & $\begin{array}{l}\text { Bis- } \\
\text { methylcyclo } \\
\text { pentadienyl- } \\
\text { Nickel }\end{array}$ & $4.6 \times 10^{-2 b}$ & & $\begin{array}{l}P_{\text {res }}: 10^{-6} \text { mbar, } \\
P_{\text {dep }}: 3 \times 10^{-5} \text { mbar, } \\
10-250 \mathrm{nA}\end{array}$ & $\begin{array}{l}12 \text { at. } \% \mathrm{Ni} \\
6 \text { at. } \% \mathrm{O} ; \\
82 \text { at. } \% \mathrm{C}\end{array}$ & 330 \\
\hline $\begin{array}{l}\mathrm{CpPt}(\mathrm{Me})_{3} \\
20 \mathrm{keV} \\
\text { electrons }\end{array}$ & $\begin{array}{l}\text { Trimethylcyclo- } \\
\text { pentadienyl } \\
\text { platinum }\end{array}$ & $\begin{array}{l}54 \text { mTorr, } \\
0.045 \text { Torr }^{\mathrm{c}}\end{array}$ & $\begin{array}{l}77.8 \\
71.2\end{array}$ & $0.5-40 \mathrm{keV}, 1-2000 \mathrm{pA}$ & $13 \%-21.5 \% \mathrm{Pt}$ & 343 and 363 \\
\hline $\begin{array}{l}\mathrm{MeCpPt}(\mathrm{Me})_{3} \\
15 \mathrm{keV} \\
\text { electrons }\end{array}$ & $\begin{array}{l}\text { Trimethyl- } \\
\text { methylcyclopenta- } \\
\text { dienyl } \\
\text { platinum }\end{array}$ & $\begin{array}{l}0.2 \text { Torr } \\
\text { at } 35^{\circ} \mathrm{C} \\
0.053 \text { Torr }^{\mathrm{c}}\end{array}$ & $\begin{array}{l}71.5 \\
43.6\end{array}$ & $\begin{array}{l}\varnothing: 3 \mathrm{~nm} ; \\
P_{\text {res }}: 10^{-9} \text { Torr } \\
P_{\text {dep }}: 10^{-6} \text { Torr } \\
10^{18}-10^{19} \text { molecule } / \mathrm{cm}^{2} \mathrm{~s}\end{array}$ & $\begin{array}{l}21.5 \text { at. } \% \mathrm{Pt}, \\
73 \text { at. } \% \mathrm{C} \\
5.5 \text { at. } \% \mathrm{O} \\
30 \mu \Omega \mathrm{cm}\end{array}$ & 364 and 365 \\
\hline $\begin{array}{l}\mathrm{MeCpPt}(\mathrm{Me})_{3} \\
25 \mathrm{keV} \mathrm{Ga}^{+} \\
\text {ions }\end{array}$ & $\cdots$ & $\begin{array}{l}\text { heated } 26-55^{\circ} \mathrm{C} \\
(0.11-0.50 \text { Torr })\end{array}$ & $\cdots$ & & $\begin{array}{l}13-21.5 \text { at. } \% \\
\mathrm{Pt}\end{array}$ & 366 \\
\hline $\begin{array}{l}\mathrm{MeCpPt}(\mathrm{Me})_{3} \\
35 \mathrm{keV} \mathrm{Ga}^{+} \\
\text {ions }\end{array}$ & $\cdots$ & $\cdots$ & $\cdots$ & Total yield: $0.2-30$ atoms/ion & $\begin{array}{l}\mathrm{Pt}: \mathrm{C}: \mathrm{Ga}: \mathrm{O}, \\
46: 24: 28: 2, \\
70-140 \mu \Omega \mathrm{cm}\end{array}$ & 171 and 367 \\
\hline $\begin{array}{l}\mathrm{MeCpPt}(\mathrm{Me})_{3} \\
30 \mathrm{keV} \mathrm{Ga}^{+} \\
\text {ions }\end{array}$ & $\cdots$ & $\cdots$ & $\cdots$ & & $\begin{array}{l}\mathrm{Pt}: \mathrm{C}: \mathrm{Ga}: \mathrm{O} \\
45: 45: 5: 5\end{array}$ & 368 \\
\hline
\end{tabular}

${ }^{\mathrm{a}}$ Reference 361.

${ }^{\mathrm{b}}$ Estimated from Ref. 362.

${ }^{\mathrm{c}}$ Reference 360.

as shown above [see Sec. V A 3 (d)]. The very high enthalpies of formation of silicon carbide $(\mathrm{SiC})$, silicon nitride $\left(\mathrm{Si}_{3} \mathrm{~N}_{4}\right)$, and especially silicon oxide $\left(\mathrm{SiO}_{2}\right)$ are a strong driving force for obtaining these compounds (with highest probability $\mathrm{SiO}_{2}$ ) instead of other compounds. Under high electron irradiation doses, however, partial oxygen desorption might occur. This would explain the non-Ohmic electrical properties mentioned by Christy for very thin films. ${ }^{8}$ These depositions with condensed films of silicone oil resulted in poor electrically insulating materials. Comparable to this are the depositions with very low volatile compounds such as octavinylsilsesquioxane condensed as films on substrates and exposed like photeresists by electrons. ${ }^{378}$ The easily polymerizable vinyl groups in this molecule are responsible for lowering the needed electron dose in order to decrease the volatility so that by heating, the compound will decompose prior to evaporate from the surface. For the discussion of the $\mathrm{SiO}_{2}$ deposits from alkoxides or the silicon isocyanate with additional gases, we refer to the next section. FEB induced deposition from titaniumtetranitrate $\left[\mathrm{Ti}\left(\mathrm{NO}_{3}\right)_{4}\right]$ resulted in titanium oxide that still contained some nitrogen. ${ }^{379} 10 \%$ of nitrogen detected by XPS and EDX seems to be present as nonreacted nitrate units as they were also detected by IR spectroscopy.

\section{Precursors for oxide deposition (dielectrics)}

In this section we summarize precursors used for oxide deposition without additional gases. Generally, carbon con- 
TABLE XVI. FEB and FIB induced deposition using acetylacetonate precursors. Vapor pressures $P_{\text {vap }}$, deposition conditions, deposit composition, and electrical resistivity are given. Values for the evaporation and sublimation enthalpies $\Delta H_{\text {vap/subl }}$ were collected from Refs. 266, 268, and 269. Other symbols are $\varnothing$ (beam diameter), $P_{\text {res }}$ (residual vacuum pressure), $P_{\text {dep }}$ (pressure during deposition or estimated local pressure on sample surface), and $t_{\text {dep }}$ (deposition time). The abbreviations for acetylacetonate, trifluoroacetonate, hexafluoroacetylacetonate are acac, tfa, and hfa, respectively. VTMS=Vinyltrimethylsilane, MHY=2-methyl-1-hexen-3-yne, DMB=3,3-dimethyl-1-butene.

\begin{tabular}{|c|c|c|c|c|c|}
\hline $\begin{array}{l}\text { Precursor } \\
\text { irradiation }\end{array}$ & $\begin{array}{l}P_{\text {vap }} \text { at } \\
\text { RT } \\
(\text { mbar })\end{array}$ & $\begin{array}{l}\Delta H_{\mathrm{vap} / \mathrm{subl}} \\
\text { at } \mathrm{RT} \\
(\mathrm{kJ} / \mathrm{mol})\end{array}$ & Conditions & $\begin{array}{l}\text { Composition } \\
\text { (at. \%) } \\
\text { resistivity }\end{array}$ & Ref. \\
\hline $\begin{array}{l}\mathrm{Me}_{2} \mathrm{Au}(\mathrm{hfa}) \\
40 \mathrm{keV} \mathrm{Ga}\end{array}$ & 0.7 & & Total yield: $3-8$ atoms/ion & $\begin{array}{l}\mathrm{Au}: \mathrm{C}: \mathrm{Ga} \\
\text { 50:35:15 (RT), } \\
500-1500 \mu \Omega \mathrm{cm} \\
\text { 80:10:10 }\left(120^{\circ} \mathrm{C}\right) \\
3-10 \mu \Omega \mathrm{cm}\end{array}$ & 58,169 , and 370 \\
\hline $\begin{array}{l}\mathrm{Me}_{2} \mathrm{Au}(\mathrm{tfa}) \\
25 \mathrm{keV} \text { electrons }\end{array}$ & 0.07 & 83.5 & $100 \mathrm{pA}-1 \mathrm{nA}$ & $\begin{array}{l}25-40 \text { at. } \% \mathrm{Au} \\
10^{3}-10^{5} \mu \Omega \mathrm{cm}\end{array}$ & 371 \\
\hline $\begin{array}{l}\mathrm{Me}_{2} \mathrm{Au}(\mathrm{tfa}) \\
25 \mathrm{keV} \text { electrons }\end{array}$ & $\cdots$ & $\cdots$ & $\begin{array}{l}\mathrm{Si}_{3} \mathrm{~N}_{4} \text { membrane at }-3{ }^{\circ} \mathrm{C} \\
\varnothing: 0.15 \mu \mathrm{m}, 10^{8} \mathrm{~A}\end{array}$ & $\begin{array}{l}40 \text { at. } \% \mathrm{Au}, \\
55 \text { at. } \% \mathrm{C} \\
1.5 \text { at. } \% \mathrm{O}\end{array}$ & 11 \\
\hline $\begin{array}{l}\mathrm{Me}_{2} \mathrm{Au}(\mathrm{tfa}) \\
2-30 \mathrm{keV} \\
\text { electrons }\end{array}$ & $\cdots$ & $\cdots$ & $\begin{array}{l}\text { Substrate at } 5{ }^{\circ} \mathrm{C}, 50 \mathrm{pA}, \\
\varnothing: 10-50 \mathrm{~nm}\end{array}$ & 25 at. $\% \mathrm{Au}$ & 372 \\
\hline $\begin{array}{l}\mathrm{Me}_{2} \mathrm{Au}(\mathrm{tfa}) \\
25 \mathrm{keV} \text { electrons }\end{array}$ & $\cdots$ & $\cdots$ & Dep. rate: $4 \mathrm{~nm} / \mathrm{s}$ & $\mathrm{Au}_{0.2} \mathrm{C}_{0.8}$ & 258 \\
\hline $\begin{array}{l}\mathrm{Me}_{2} \mathrm{Au}(\mathrm{tfa}) \\
20 \mathrm{keV} \text { electrons }\end{array}$ & $\cdots$ & $\cdots$ & $1 \mathrm{pA}-10 \mathrm{nA}$ & $\begin{array}{l}25-40 \text { at. } \% \mathrm{Au} \\
10^{3}-10^{5} \mu \Omega \mathrm{cm}\end{array}$ & 373 \\
\hline $\begin{array}{l}\mathrm{Me}_{2} \mathrm{Au}(\mathrm{acac}) \\
200 \mathrm{keV} \text { electrons }\end{array}$ & $\cdots$ & $\cdots$ & $\begin{array}{l}50 \mathrm{~nm} \mathrm{Si}_{3} \mathrm{~N}_{4} \text { membrane, } \\
\varnothing: 1-4 \mathrm{~nm}, P_{\text {res }}: 10^{-7} \text { Torr, } \\
P_{\text {dep }}: 10^{-5} \text { Torr, } 1-5 \mathrm{pA}\end{array}$ & Contains carbon & 374 \\
\hline $\begin{array}{l}(\mathrm{tfa}) \mathrm{Cu}-\mathrm{VTMS} \\
25 \mathrm{keV} \text { electrons }\end{array}$ & 0.1 & $\cdots$ & $90 \mathrm{pA}$ & $\mathrm{Cu}_{0.1} \mathrm{C}_{0.5} \mathrm{O}_{0.1} \mathrm{Si}_{0.3}$ & 258 \\
\hline $\begin{array}{l}\text { (tfa)Cu-VTMS } \\
25 \mathrm{keV} \text { electrons }\end{array}$ & $\cdots$ & $\cdots$ & $\begin{array}{l}\text { Freestanding deposit, } \\
\varnothing: 132 \mathrm{~nm}, 500 \mathrm{pA}\end{array}$ & $\begin{array}{l}\text { Pure } \mathrm{Cu} \\
\text { (FEB heating) }\end{array}$ & 212 \\
\hline $\begin{array}{l}\text { (tfa)Cu-VTMS } \\
25 \mathrm{keV} \text { electrons }\end{array}$ & $\cdots$ & $\cdots$ & Si substrate, $0.5-1 \mathrm{nA}$ & $\begin{array}{l}20-45 \text { at. } \% \mathrm{Cu}, \\
35-70 \text { at. } \% \mathrm{C}, \\
8-14 \text { at. } \% \mathrm{O}, \\
2-10 \text { at. } \% \mathrm{Si}, \\
5-11 \text { at. } \% \mathrm{Cu}\end{array}$ & 182 and 240 \\
\hline $\begin{array}{l}\text { (hfa)Cu-VTMS } \\
30 \text { and } 50 \mathrm{keV} \text { electrons }\end{array}$ & $0.4 / 40{ }^{\circ} \mathrm{C}$ & $\cdots$ & $\cdots$ & $8-11$ at. $\% \mathrm{Cu}$ & 375 \\
\hline $\begin{array}{l}(\mathrm{hfa}) \mathrm{Cu}-\mathrm{VTMS} \\
25-35 \mathrm{keV} \mathrm{Ga}^{+} \text {ions }\end{array}$ & $\cdots$ & $\cdots$ & Total yield: $10-30$ atoms/ion & $\begin{array}{l}\mathrm{Cu}: \mathrm{C}, \\
55: 45\left(25^{\circ} \mathrm{C}\right), \\
100 \mu \Omega \mathrm{cm}, \\
95: 5\left(100{ }^{\circ} \mathrm{C}\right), \\
5 \mu \Omega \mathrm{cm}\end{array}$ & 376 \\
\hline $\begin{array}{l}(\mathrm{hfa}) \mathrm{Cu}-\mathrm{VTMS} \\
50 \mathrm{keV} \mathrm{Ga}{ }^{+} \text {ions }\end{array}$ & $\cdots$ & $\cdots$ & $\begin{array}{l}49-2070 \mathrm{pA}, 0.33 \mu \mathrm{m}^{3} / \mathrm{nC} \text {, best quality: } \\
0.18 \mu \mathrm{m}^{3} / \mathrm{nC}\end{array}$ & $\begin{array}{l}\mathrm{Cu}: \mathrm{C}: \mathrm{Ga} \\
60: 30: 10(\mathrm{RT}) \\
18.8 \mu \Omega \mathrm{cm} \\
\left(100^{\circ} \mathrm{C}\right)\end{array}$ & 377 \\
\hline $\begin{array}{l}\mathrm{Cu}(\mathrm{hfa})_{2} \\
\mathrm{Cu}(\mathrm{hfa})_{2} x \mathrm{H}_{2} \mathrm{O} \\
25 \mathrm{keV} \text { electrons }\end{array}$ & $4 \times 10^{-3}$ & $\begin{array}{l}97 \\
108 \pm 6\end{array}$ & Si substrate, $0.5-1 \mathrm{nA}$ & $\begin{array}{l}14 \text { at. } \% \mathrm{Cu}, \\
75 \text { at. } \% \mathrm{C}, \\
5 \text { at. } \% \mathrm{O}\end{array}$ & 240 \\
\hline $\begin{array}{l}\text { (hfa)CuMHY } \\
25 \mathrm{keV} \text { electrons }\end{array}$ & 0.2 & $\cdots$ & Si substrate, $0.5-1 \mathrm{nA}$ & $\begin{array}{l}13 \text { at. } \% \mathrm{Cu}, \\
82 \text { at. } \% \mathrm{C} \\
3 \text { at. } \% \mathrm{O}\end{array}$ & 240 \\
\hline $\begin{array}{l}\text { (hfa)CuDMB } \\
25 \mathrm{keV} \text { electrons }\end{array}$ & 1.3 & $\cdots$ & Si substrate, $0.5-1 \mathrm{nA}$ & $\begin{array}{l}25-60 \text { at. } \% \mathrm{Cu} \\
15-60 \text { at. } \% \mathrm{C} \\
5-25 \text { at. } \% \mathrm{O}\end{array}$ & 240 \\
\hline
\end{tabular}

tamination of the deposit is found for carbon containing precursors, which is, however, at an acceptable limit for certain applications, like the electrical insulating properties of the $\mathrm{SiO}_{2}$ containing deposits. Table XVII summarizes experiments performed for the oxides of $\mathrm{Si}$, $\mathrm{Ti}$, and $\mathrm{Ta}$.

\section{Postdeposition treatments}

Deposits can be post-treated; in most cases thermal annealing is carried out. The annealing can take place either in vacuum or in the presence of reactive gases that might be 
TABLE XVII. FEB and FIB induced deposition of oxides. Vapor pressures $P_{\text {vap }}$, deposition conditions, and the deposit compositions are given. Structural formulas are given for identification purposes. Other symbols are $\varnothing$ (beam diameter), $P_{\text {res }}$ (residual vacuum pressure), $P_{\text {dep }}$ (pressure during deposition or estimated local pressure on sample surface), and $t_{\text {dep }}$ (deposition time).

\begin{tabular}{|c|c|c|c|c|c|}
\hline $\begin{array}{l}\text { Precursor } \\
\text { formula } \\
\text { irradiation }\end{array}$ & $\begin{array}{l}\text { Structural } \\
\text { formula }\end{array}$ & $\begin{array}{l}P_{\text {vap }} \text { at RT } \\
\text { (mbar) }\end{array}$ & Conditions & $\begin{array}{l}\text { Composition } \\
\text { Resistivity }\end{array}$ & Ref. \\
\hline $\begin{array}{l}\text { TEOS } \\
\mathrm{Si}(\mathrm{OEt})_{4} \\
25 \mathrm{keV} \text { electrons }\end{array}$ & & $1.7 / 25^{\circ} \mathrm{C}$ & $\begin{array}{l}P_{\text {res }}: 5 \times 10^{-7} \text { mbar, } \\
90 \mathrm{pA}, \\
10^{18} \text { molecules } / \mathrm{cm}^{2} \mathrm{~s}\end{array}$ & $\mathrm{Si}_{0.15} \mathrm{C}_{0.45} \mathrm{O}_{0.4}$ & 258 \\
\hline $\begin{array}{l}\text { TEOS } \\
\mathrm{Si}(\mathrm{OEt})_{4} \\
30 \mathrm{keV} \mathrm{Ga}^{+} \text {ions }\end{array}$ & & $1.7 / 25^{\circ} \mathrm{C}$ & $\begin{array}{l}P_{\text {res }}: 10^{-6} \text { Torr } \\
40-2000 \mathrm{pA}\end{array}$ & $10^{8} \Omega \mathrm{cm}$ & 380 \\
\hline $\begin{array}{l}\text { Octavinylsilsesquioxane } \\
\left(\mathrm{C}_{2} \mathrm{H}_{3} \mathrm{SiO}_{1.5}\right)_{8}=\mathrm{Si}_{8} \mathrm{O}_{12} \mathrm{C}_{16} \mathrm{H}_{24} \\
5-50 \mathrm{keV} \text { electrons }\end{array}$ & & $\begin{array}{c}\text { At } 5 \times 10^{-5} \text { mbar } \\
\text { the resist } \\
\text { sublimes at } \\
180{ }^{\circ} \mathrm{C}\end{array}$ & & $\begin{array}{l}\text { Index of } \\
\text { refraction, } 1.48 \\
\text { (no wavelength } \\
\text { specified) }\end{array}$ & 378 \\
\hline $\begin{array}{l}\text { Octamethylcyclotetrasiloxane } \\
{\left[\left(\mathrm{CH}_{3}\right)_{2} \mathrm{SiO}\right]_{4}=\mathrm{Si}_{4} \mathrm{O}_{4} \mathrm{C}_{8} \mathrm{H}_{24}} \\
50 \mathrm{keV} \mathrm{Ga}^{+} \text {ions }\end{array}$ & & 1.4 & $\begin{array}{l}\text { Beam current, } 10 \mathrm{nA} \text {; } \\
\text { dwell time, } 100 \mathrm{~ns} \text {; } \\
\text { pixel spacing, } 400 \mathrm{~nm} \text {; } \\
\text { siloxane } \\
\text { pressure } \sim 0.3 \text { Torr } \\
\text { in the delivery line! } \\
\text { oxygen pressure } \\
3.5 \text { Torr, }\end{array}$ & $\begin{array}{c}\text { Si:O:Ga, } \\
27: 56: 17 \\
1.2 \times 10^{7} \Omega \mathrm{cm}\end{array}$ & 381,382 \\
\hline $\begin{array}{l}\text { Pentamethylcyclopentasiloxane } \\
{\left[\left(\mathrm{CH}_{3}\right) \mathrm{HSiO}_{5}=\mathrm{Si}_{5} \mathrm{O}_{5} \mathrm{C}_{5} \mathrm{H}_{20}\right.} \\
50 \mathrm{keV} \mathrm{Ga}{ }^{+} \text {ions }\end{array}$ & & $\begin{array}{c}13 \\
\text { at } 54^{\circ} \mathrm{C}\end{array}$ & $\cdots$ & $8 \times 10^{11} \Omega \mathrm{cm}$ & 382 \\
\hline $\begin{array}{l}\text { Dodecamethylpentasiloxane } \\
=\mathrm{Si}_{5} \mathrm{O}_{4} \mathrm{C}_{12} \mathrm{H}_{36} \\
50 \mathrm{keV} \mathrm{Ga}^{+} \text {ions }\end{array}$ & 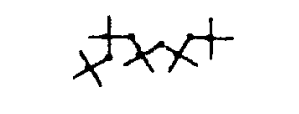 & $\begin{array}{c}0.007 \\
\text { (extrapol.) }\end{array}$ & $\cdots$ & & 382 \\
\hline $\begin{array}{l}\text { Tetrakis(dimethylsiloxy) silane } \\
{\left[\mathrm{HSi}\left(\mathrm{CH}_{3}\right)_{2} \mathrm{O}\right]_{4} \mathrm{Si}} \\
50 \mathrm{keV} \mathrm{Ga} \\
\text { ions }\end{array}$ & 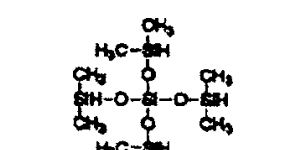 & Not found & $\cdots$ & $3 \times 10^{10} \Omega \mathrm{cm}$ & 382 \\
\hline $\begin{array}{l}\mathrm{H}_{3} \mathrm{C}\left(-\mathrm{O}-\mathrm{Si}\left(\mathrm{CH}_{3}\right)\left(\mathrm{C}_{6} \mathrm{H}_{5}\right)\right)_{x}-\mathrm{O}-\mathrm{CH}_{3} \\
0.225 \mathrm{keV} \text { electrons }\end{array}$ & $\begin{array}{l}\text { Methyl-, phenylsiloxane } \\
\text { (silicone oil, Dow } \\
\text { Corning 704) }\end{array}$ & $\begin{array}{l}\text { Estimation } \\
\text { in article } \\
2 \times 10^{-9} \text { Torr }\end{array}$ & $P_{\text {res }}: 4 \times 10^{-6}-2 \times 10^{-5}$ Torr & Not measured & 8 \\
\hline $\begin{array}{l}\mathrm{Ta}(\mathrm{OEt})_{5} \\
35 \mathrm{keV} \mathrm{Ga}{ }^{+} \text {ions, } \\
50 \mathrm{keV} \mathrm{He}^{+}, \mathrm{Ar}^{+}, \\
\mathrm{Xe}^{+}\end{array}$ & & $\begin{array}{l}2.6 \times 10^{-4} \text { mbar } \\
\quad(\text { extrapol. })\end{array}$ & $\begin{array}{l}\text { Focused beams: } 10-100 \mathrm{~mA} / \mathrm{cm}^{2} \text {, } \\
\text { broad beams: } 1 \mu \mathrm{A} / \mathrm{cm}^{2}\end{array}$ & $\begin{array}{l}10: 3: 5 \\
\mathrm{Ta}: \mathrm{C}: \mathrm{O}\end{array}$ & 383 \\
\hline $\begin{array}{l}\mathrm{Ti}(\mathrm{O}-i \text {-prop })_{4} \\
25 \mathrm{keV} \text { electrons }\end{array}$ & & $\begin{array}{l}0.27 \text { mbar } \\
\text { at } 30^{\circ} \mathrm{C}\end{array}$ & $\begin{array}{l}P_{\text {res }}: 10^{-6} \text { mbar, } \\
P_{\text {dep }}: 3 \times 10^{-5} \text { mbar, } \\
10-250 \mathrm{nA}\end{array}$ & $\begin{array}{c}\text { index of refraction } \\
\text { of } 3.3 \text { at } 500 \mathrm{~nm} \text {; } \\
\text { Ti } 34 \text { at. } \% \text {, O } 51 \text { at. } \% \text {, } \\
\text { C } 15 \text { at. } \%\end{array}$ & 384 \\
\hline $\begin{array}{l}\mathrm{Ti}\left(\mathrm{NO}_{3}\right)_{4} \\
25 \mathrm{keV} \text { electrons }\end{array}$ & & $\begin{array}{c}\text { Sublimes } \\
\text { easily at } 0.2 \text { Torr, } \\
50{ }^{\circ} \mathrm{C}\end{array}$ & $\begin{array}{l}P_{\text {res }}: 10^{-6} \text { mbar, } \\
P_{\text {dep }}: 3 \times 10^{-5} \text { mbar, } \\
10-250 \mathrm{nA}\end{array}$ & $\begin{array}{c}35 \% \mathrm{Ti} \\
57 \pm 5 \% \mathrm{O} \\
8.5 \pm 5 \% \mathrm{~N}\end{array}$ & 379 \\
\hline
\end{tabular}

oxidizing or reducing. This section will not cover all of the tests carried out in this field. Generally, annealing of FEB or FIB deposited materials follows in analogy to annealing of materials obtained by CVD or physical vapor deposition. As diffusion processes and chemical reactions on surfaces and in the deposit volume determine the "purification" and transformation, there is no difference to such annealing experiments reported and well established. As an example, thermal annealing under vacuum $\left(1 \mathrm{~h}, 600{ }^{\circ} \mathrm{C}\right.$ of FEB deposits from $\mathrm{Fe}(\mathrm{CO})_{5}$ containing amorphous iron and carbon resulted in a crystalline mixture of $\alpha$-iron and different carbides. ${ }^{339}$ An example of oxidative purification is shown in Fig. 37, where annealing in air results in a visible volume reduction and the appearance of gold particles from the composite material.

More voluminous deposits have been annealed at $500{ }^{\circ} \mathrm{C}$ in oxygen atmosphere and resulted in improved purity of platinum and gold from the commercially available precursors $\mathrm{MeCpPt}(\mathrm{Me})_{3}$ and $\mathrm{Me}_{2} \mathrm{Au}(\mathrm{acac}),{ }^{385}$ respectively.

Postannealing of $\mathrm{C}$ deposits from formic acid under vacuum reduced the film thickness by a factor of 2 and increased the carbon content to a final C:O ratio of 98:2. The film was more graphitic after the annealing. ${ }^{269}$ 

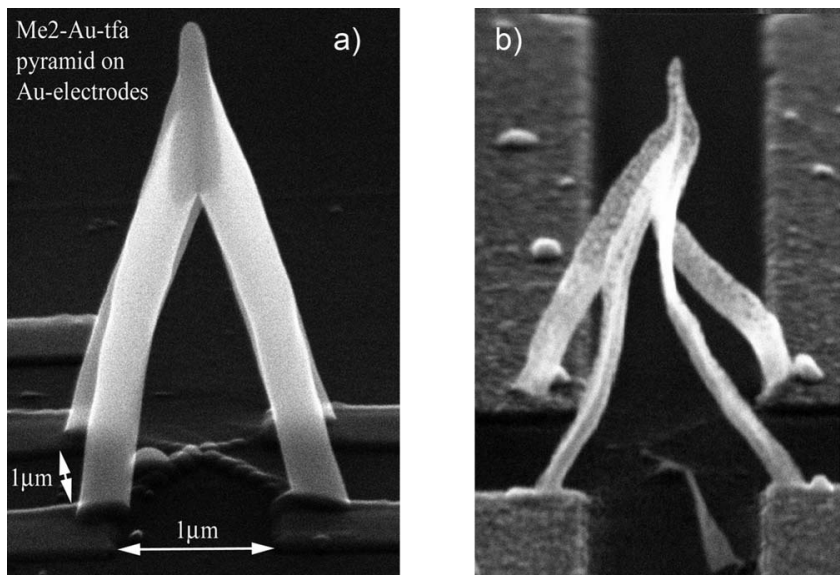

FIG. 37. SEM image of a FEB deposited pyramid connecting four predeposited $\mathrm{Au}$ electrodes. The precursor was $\mathrm{Me}_{2} \mathrm{Au}(\mathrm{tfa})$ and the electron beams $25 \mathrm{keV}$ and $200 \mathrm{pA}$. (a) As-deposited composite Au-carbonaceous matrix. (b) After thermal annealing at $400{ }^{\circ} \mathrm{C}$ for $30 \mathrm{~min}$ in air. Note the formation of large visible Au grains due to removal of carbon by oxidation. The distance of the electrodes is $1 \mu \mathrm{m}$.

\section{Precursors and additional reactive gases}

All carbon containing precursors result in carbonaceous codeposits as long as the substrate temperature is below the desorption temperature of the ligands. In the case of higher temperatures the carbon contamination is determined by the competition of ligand fragmentation rate and ligand desorption rate. As the precursor decomposition temperature and the ligand desorption temperature from the corresponding metal surface are generally very close, ligands from room temperature stable precursors will not readily desorb from substrates at room temperature. Therefore the contamination with carbon from carbon containing precursors is almost unavoidable with substrates kept at room temperature or lower. Organometallics, including compounds with aromatic ligands and the large family of $\beta$-diketonates with charged metal ions, generally result therefore in highly carbon contaminated deposits. Approaches to decrease the carbon content by adding gases or changing substrate temperature are partially successful for noble metals and pure oxide deposition. Thus the purity of the carbon containing deposits can be improved by adding chemically reactive gases to oxidize or reduce the carbon during the deposition process. As conceptually outlined in Sec. III F, the action of a reactive gas adsorbate will depend heavily on the process regime that can be installed for the reaction of oxidation or reduction with respect to the process regime of general molecule fragmentation (and fixation). Here the successful results for noble metals and for pure oxides and nitrides are presented.

\section{Metals, metal oxides, and metal nitrides}

Adding oxidizing gases for deposition of pure gold $(\mathrm{Au})$ works reasonably well as shown in an ESEM that has not been specifically cleaned before deposition with dimethylgold acetylacetonate $\left[\mathrm{Me}_{2} \mathrm{Au}(\mathrm{acac})\right]$ as precursor. ${ }^{386,387}$ Adding an $\mathrm{Ar} / \mathrm{O}_{2}$ mixture or water vapor both increased the gold purity up to 50 and 20 at. \%, respectively. A more detailed study revealed the appearance of polycrystalline pure gold nanoparticles in the central part of the deposits surrounded by a carbonaceous crust surrounding the pure gold deposits. The deposits were obtained in the presence of sufficient water vapor together with the precursor $\mathrm{Me}_{2} \mathrm{Au}(\mathrm{acac}) .^{388}$ For the deposition of almost pure copper, atomic hydrogen was used as additional gas to $\mathrm{Ar}$ ion FIBID with (hfa)Cu-VTMS ${ }^{389}$

The FEBID with the aromatic sandwich compound bis(methylcyclopentadienyl)-Ni(II) resulted in a strong decrease in the carbon content of the deposits from $80 \%$ down to about $50 \%$, but no significant improvement in electrical conductivity was obtained due to the formation of nickel oxides. The addition of hydrogen gas into the chamber mainly desorbed higher quantities of water from the microscope walls and again only an increased oxide content was observed but no reductive action of the added hydrogen gas. ${ }^{330}$ Magnetic oxide, such as magnetite, was deposited recently for data storage research. The addition of water vapor to iron carbonyl up to 1.5 times the flow of precursor resulted in the reduction of the carbon content in the FEBID product from $25 \%$ to $0 \%$. By this crystalline magnetite, $\mathrm{Fe}_{3} \mathrm{O}_{4}$ could be obtained. ${ }^{391}$

Using nitrogen radicals as reactive gas and gallane quinuclidine as precursor for FIB deposition, GaN semiconductor nanostructures could be obtained at $600{ }^{\circ} \mathrm{C} .{ }^{392}$ Below $300{ }^{\circ} \mathrm{C}$ the deposits show strong $\mathrm{C}$ contamination. The data are summarized in Table XVIII.

\section{Pure silicon dioxide $\left(\mathrm{SiO}_{2}\right)$}

Main group or transition metal oxides are either electrically insulating or semiconducting materials, and more or less optically transparent. Oxides, either as thin films (antireflexion coatings) or as ceramics (alumina and yttria) or monocrystalline (sapphire), are long term stable materials and are widely applied in industry. Historically silicon oxide is the most important electrically insulating material and also highly optically transparent in a wide range of optical wavelength (190-1600 nm). Higher index of refraction (higher dielectric constant) materials were also studied in beam induced deposition, but less frequently. Again as in the case of gas-assisted metal deposition, the deposit chemical composition is strongly influenced by the residual gases in the reaction chamber.

The deposition of high quality dielectrics has mainly been studied for DUV transparent materials and is therefore focused on $\mathrm{SiO}_{2}$. Three classes of precursors have been studied, silanes, alkoxysilanes, and alkyl-aryl silanes. All of them might result in pure $\mathrm{SiO}_{2}$ by adding sufficient "oxidizing" gases such as water, oxygen, or $\mathrm{N}_{2} \mathrm{O}$. Differences between these precursors are the sticking behavior on substrates and the sensitivity to water. For the alkoxides the combination of the water sensitivity and the resulting high sticking coefficient might explain the high FEBID yields and the large amounts of oxygen needed for complete oxidation of the carbonaceous codeposits. ${ }^{393}$ The unsaturated vinyl-Si precursor could additionally combine a polymerization-type reac- 
TABLE XVIII. FEB and FIB induced deposition of metals and metal oxides with additional reactive gases. Vapor pressures $P_{\text {vap }}$, deposition conditions, and the deposit compositions are given. ESEM stands for environmental scanning electron microscope and UHV-SEM for ultrahigh vacuum scanning electron microscope.

\begin{tabular}{|c|c|c|c|c|c|}
\hline $\begin{array}{l}\text { Precursor } \\
\text { irradiation }\end{array}$ & Added gas & $\begin{array}{l}P_{\text {vap at }} \text { at } \\
\text { RT } \\
\text { (mbar) }\end{array}$ & Conditions & Composition & Ref. \\
\hline $\begin{array}{l}\mathrm{Me}_{2} \mathrm{Au}(\mathrm{acac}) \\
10 \mathrm{keV} \text { electrons }\end{array}$ & $\begin{array}{c}\mathrm{H}_{2} \mathrm{O} \\
\mathrm{O}_{2}\end{array}$ & $0.013 / 25^{\circ} \mathrm{C}$ & ESEM & $\begin{array}{l}\text { Pure Au core } \\
\text { embedded in carbon } \\
\text { crust }\end{array}$ & 388 \\
\hline $\begin{array}{l}\mathrm{Me}_{2} \mathrm{Au}(\mathrm{hfa}) \\
10-30 \mathrm{keV} \\
\text { electrons }\end{array}$ & $\begin{array}{c}\left(80 \% \mathrm{Ar} / 20 \% \mathrm{O}_{2}\right) \\
\mathrm{H}_{2} \mathrm{O} \\
\mathrm{Ar}\end{array}$ & 0.933 & $\begin{array}{l}\text { Environmental subcell } \\
\text { inside SEM and } \\
\text { ESEM }\end{array}$ & $\begin{array}{l}<12 \text { at } \% \text { (no added gas) } \\
15 \text { at } \%(\mathrm{Ar}) \\
50 \text { at } \% \mathrm{Au} \\
\left(80 \% \mathrm{Ar} / 20 \% \mathrm{O}_{2}\right) \\
20 \text { at } \% \mathrm{Au}\left(\mathrm{H}_{2} \mathrm{O}\right)\end{array}$ & 386 and 387 \\
\hline $\begin{array}{l}\mathrm{Fe}(\mathrm{CO})_{5} \\
30 \mathrm{keV} \text { electrons }\end{array}$ & $\mathrm{Fe}\left(\mathrm{C}_{5} \mathrm{H}_{5}\right)_{2}$ & 47 & $\begin{array}{l}\text { UHV-SEM, ratio: } \\
\text { carbonyl/(carbonyl } \\
\text { +cyclopentadienyl): } \\
0.66-0.9\end{array}$ & $\begin{array}{l}\text { From } \\
\mathrm{Fe}_{0.7} \mathrm{O}_{0.1} \mathrm{C}_{0.2} \\
\text { to } 30 \text { at } \% \mathrm{Fe}\end{array}$ & 390 \\
\hline $\begin{array}{l}\mathrm{Fe}(\mathrm{CO})_{5} \\
30 \mathrm{keV} \text { electrons }\end{array}$ & $\mathrm{H}_{2} \mathrm{O}$ & 47 & $\begin{array}{l}\text { UHV-SEM, } \\
\text { ratio precursor/water: } \\
0-1.5\end{array}$ & $\begin{array}{l}\text { From } \\
\mathrm{Fe}_{1} \mathrm{O}_{0.55} \mathrm{C}_{0.45} \\
\text { to cryst. } \mathrm{Fe}_{3} \mathrm{O}_{4} \\
\text { (pure oxide!) }\end{array}$ & 391 \\
\hline $\begin{array}{l}\mathrm{Ni}(\mathrm{MeCp})_{2} \\
10-25 \mathrm{keV} \\
\text { electrons }\end{array}$ & $\mathrm{H}_{2}$ & $5 \times 10^{-2}$ & $\begin{array}{l}P_{\text {res }}: 10^{-6} \text { mbar, } \\
P_{\text {dep }}: 3 \times 10^{-5} \text { mbar, } \\
10-250 \mathrm{nA}\end{array}$ & $\begin{array}{l}12 \text { at } \% \mathrm{Ni} \\
6 \text { at } \% \mathrm{O} \\
82 \text { at } \% \mathrm{C}\end{array}$ & 330 \\
\hline $\begin{array}{l}\text { (tfa)Cu-VTMS } \\
0.5 \mathrm{keV} \mathrm{Ar}^{+}\end{array}$ & Atomic $\mathrm{H}$ & 0.1 & $\begin{array}{l}\text { UHV system, } \\
\text { ratio: } \mathrm{H} / \mathrm{Ar}=3000: 1\end{array}$ & $\begin{array}{l}99 \text { at } \% \mathrm{Cu} \text {, } \\
\text { carbon etch yield } \\
\text { enhancement: } 5\end{array}$ & 389 \\
\hline $\begin{array}{l}\mathrm{GaH}_{3}: \mathrm{NC}_{7} \mathrm{H}_{14} \\
25 \mathrm{keV} \mathrm{Ga}\end{array}$ & $\mathrm{N}$ radicals & & $600{ }^{\circ} \mathrm{C}$ substrate & $\mathrm{GaN}$ & 392 \\
\hline
\end{tabular}

tion that would privilege $\beta$-unsaturated silanes as precursors. Interestingly, tetramethylsilane (a nonsticking, relatively moisture stable, and oxygen insensitive compound) results in pure $\mathrm{SiO}_{2}$ if a coflux of oxygen is provided. Both FIB (Ref. 382) and FEB depositions are reported in literature and recently excellent quality of transparent and fully industrially reliable DUV mask compatible material deposits is obtained. $^{352,353}$ It was found that adding more oxidizing gases such as water or oxygen decrease generally the carbon content in the deposits. ${ }^{352}$ The amount of oxygen needed to obtain carbon-free deposits depends on the precursor molecules, the total precursor flux but less on the irradiation conditions. No carbon codeposition is obtained during FEB induced deposition of $\mathrm{SiO}_{2}$ from disilane as precursor assisted with oxygen as shown by Nakano. ${ }^{280}$ For comparison a hydrogen-free precursor was tested: the presence of water and large addition of oxygen resulted in FEB deposition of pure $\mathrm{SiO}_{2}$, still containing some hydrogen atoms as detected by ERDA. ${ }^{353}$ A Ti-based material containing no carbon with a high-refractive index of 2.1 was obtained by FEB induced deposition with titanium nitrate as precursor. ${ }^{379}$ This asdeposited nonabsorbing material obtained by FEBID is very attractive for nanophotonic structures designed for visible light (Table XIX).

\section{Etchants}

A limited number of precursors exists for FEB induced etching because the dissociation and reaction products should not result in any nonvolatile solid at room temperature. Therefore, the selection of the elements is limited to hydrogen, the halogens, oxygen, nitrogen, and the noble gases. Gas-assisted FIB etching on the other hand allows the use of carbon containing etchant gases because any probable nonvolatile carbon contamination will be simultaneously sputtered off by the ions. In the following two sections we discuss gas-assisted FEB and FIB induced etching.

\section{FEB gas-assisted etching}

FEB induced etching ideally triggers the release of an etchant compound by irradiation. The compound delivers atoms, ions, or fragments that react with the solid substrate material and result in volatile reaction products. Ideally the etch reaction starts when the adsorbed molecule is being dissociated or activated by the electron beam rather than that the introduced molecule spontaneously etches the substrate. With hydrogen several tests have been carried out with more or less convincing results. Hydrogen was added in an attempt to etch codeposited carbon from the $\mathrm{CpNi}$ precursor. ${ }^{330} \mathrm{How}-$ ever, due to the residual water background pressure of the microscope, the addition of hydrogen seems to desorb water from the microscope walls and instead oxidation of the nickel deposit occurred that is opposed to the intended reduction and higher purity of nickel deposition.

Historically, electron beam induced etching concerned mainly carbon. Carbon can be etched by electron irradiation in the presence of water, ${ }^{5}$ a fact that is detrimental for elec- 
TABLE XIX. FEB induced deposition of pure oxides with additional reactive gases. Vapor pressures $P_{\text {vap }}$, deposition conditions, and the deposit compositions are given. Compositions marked with (no g.) were obtained without additional gas. Other symbols are $\varnothing$ (beam diameter), $P_{\text {res }}$ (residual vacuum pressure), $P_{\text {dep }}$ (pressure during deposition or estimated local pressure on sample surface), and $t_{\text {dep }}$ (deposition time). UHV-SEM stands for ultrahigh vacuum scanning electron microscope.

\begin{tabular}{|c|c|c|c|c|c|}
\hline $\begin{array}{l}\text { Precursor } \\
\text { irradiation }\end{array}$ & Added gas & $\begin{array}{l}P_{\text {vap }} \text { at } \\
\text { RT } \\
\text { (mbar) }\end{array}$ & Conditions & Composition & Ref. \\
\hline $\begin{array}{l}\text { Disilane } \\
\mathrm{Si}_{2} \mathrm{H}_{6} \\
0.6-17 \mathrm{keV} \\
\text { electrons }\end{array}$ & $\begin{array}{c}\mathrm{O}_{2} \\
\mathrm{O}_{2} / \mathrm{Si}_{2} \mathrm{H}_{6} \\
0.5-2.5\end{array}$ & & $\begin{array}{l}\mathrm{Si} \text {, preheated to } 900^{\circ} \mathrm{C} \text {, } \\
\text { for } 5 \mathrm{~min}, 0.27-2.7 \mathrm{~mA} / \mathrm{cm}^{2} \text {, } \\
\varnothing: 180-250 \mu \mathrm{m} \text {, } \\
P_{\text {res }}: 2 \times 10^{-10} \text { Torr; } \\
P_{\text {dep }}: 1.2 \times 10^{-5} \text { Torr }\end{array}$ & No information & 280 \\
\hline $\begin{array}{l}\text { (Mono)silane } \\
\mathrm{SiH}_{4} \\
3 \mathrm{keV} \text { electrons }\end{array}$ & $\begin{array}{c}\mathrm{N}_{2} \mathrm{O} \\
\mathrm{SiH}_{4}: \mathrm{N}_{2} \mathrm{O}=1: 75\end{array}$ & 0.33 & $\begin{array}{l}400 \mathrm{~mA} / \mathrm{cm}^{2}, \varnothing: 180 \mu \mathrm{m}, \\
P_{\text {res }}: 10^{-6} \text { Torr; } \\
P_{\text {dep }}: 1.2 \times 10^{-5} \text { Torr }\end{array}$ & $\begin{array}{l}\text { Index of } \\
\text { refraction, } 1.462 ; \\
5 \% \text { close to } \\
\text { stoichiometric value; } \\
\text { BHF etch rate, } 3.6-6 \mathrm{~nm} / \mathrm{s} \text {, } \\
\text { three to six times } \\
\text { faster than thermally } \\
\text { grown oxide }\end{array}$ & 278 \\
\hline $\begin{array}{l}\mathrm{Si}(\mathrm{OEt})_{4} \\
\text { TEOS } \\
10 \mathrm{keV} \text { electrons }\end{array}$ & $\begin{array}{c}\mathrm{O}_{2} / \mathrm{TEOS}, \\
0-15\end{array}$ & 2.2 & $\begin{array}{l}P_{\text {res }}: 5 \times 10^{-7} \mathrm{mbar} \\
125 \mathrm{pA} ; \varnothing: 800 \mathrm{~nm}\end{array}$ & $\begin{array}{l}\mathrm{SiO}_{2.5} \mathrm{C}_{3.2} \text { (no g.), } \\
\mathrm{SiO}_{2}\end{array}$ & 352 and 393 \\
\hline $\begin{array}{l}\mathrm{Si}(\mathrm{OMe})_{4} \\
\text { TMOS } \\
10 \mathrm{keV} \text { electrons }\end{array}$ & $\begin{array}{l}\mathrm{O}_{2} / \mathrm{TEOS}, \\
\quad 0-0.6\end{array}$ & 16 & $\begin{array}{l}P_{\text {res }}: 5 \times 10^{-7} \mathrm{mbar} \\
125 \mathrm{pA} ; \varnothing: 800 \mathrm{~nm}\end{array}$ & $\begin{array}{l}\mathrm{SiO}_{2.5} \mathrm{C}_{3.2} \text { (no g.), } \\
\mathrm{SiO}_{2}\end{array}$ & 352 and 393 \\
\hline $\begin{array}{l}\mathrm{Si}(\mathrm{OMe})_{4} \\
\mathrm{TMOS} \\
60 \mathrm{keV} \mathrm{Si}{ }^{2+} \\
\text { ions }\end{array}$ & $\begin{array}{c}\mathrm{O}_{2} / \mathrm{TMOS} \\
1: 2\end{array}$ & 16 & $\begin{array}{l}P_{\text {res }}: 1.3 \times 10^{-4} \mathrm{~Pa} \text {; } \\
P_{\text {dep }}: 8 \times 10^{-4} \mathrm{~Pa} \text {; } \\
\text { substrate temp.: RT- } \\
200^{\circ} \mathrm{C}\end{array}$ & $\begin{array}{l}\mathrm{SiO}_{x}, \\
x=0.5-2 \text { (no g.), } \\
x=1-2 \text { with } \mathrm{O}_{2}\end{array}$ & 394 \\
\hline $\begin{array}{l}\text { Tetramethylsilane } \\
\mathrm{Si}(\mathrm{Me})_{4} \\
5,10 \text {, and } 25 \mathrm{keV} \\
\text { electrons }\end{array}$ & $\begin{array}{c}\mathrm{O}_{2} / \mathrm{TMS} \\
0-1.7\end{array}$ & 887 & $\begin{array}{l}P_{\text {res }}: 5 \times 10^{-7} \text { mbar, } \\
5 \mathrm{kV} ; 125 \mathrm{pA} ; \varnothing:>1 \mu \mathrm{m}, \\
10 \mathrm{kV} ; 125 \mathrm{pA} ; \varnothing: 800 \mathrm{~nm}, \\
25 \mathrm{kV} ; 125 \mathrm{pA} ; \varnothing: 80 \mathrm{~nm}\end{array}$ & $\begin{array}{l}\mathrm{SiO}_{1.8} \mathrm{C}_{3.7} \text { (no g.) } \\
\mathrm{SiO}_{2}\end{array}$ & 352 and 393 \\
\hline $\begin{array}{l}\text { Tetraisocyanatosilane } \\
\mathrm{Si}(\mathrm{NCO})_{4} \\
10 \mathrm{keV} \text { electrons }\end{array}$ & $\begin{array}{c}\mathrm{O}_{2} / \text { TIS } \\
0-470\end{array}$ & 1.3 & $\begin{array}{l}P_{\text {res }}: 5 \times 10^{-7} \mathrm{mbar} \\
125 \mathrm{pA} ; \varnothing: 800 \mathrm{~nm}\end{array}$ & $\begin{array}{l}\mathrm{SiC}_{2} \mathrm{NO}_{3} \text { (no g.) } \\
\mathrm{SiO}_{2}\end{array}$ & 352 and 393 \\
\hline
\end{tabular}

tron beam observation of (frozen) hydrated biological samples. ${ }^{395}$ Etching of carbon in the form of synthetic diamond was carried out with oxygen, hydrogen, water, and air as precursor. Oxygen proves to be the most efficient etchant for diamond; the formation of amorphous carbon does not take place. ${ }^{384,396,397}$

The most prominent and industrially applied etchant is the difluoride of xenon $\left(\mathrm{XeF}_{2}\right)$, one of the few at ambient temperature stable chemical compounds containing a noble gas. Its use for FEB induced etching of silicon based material has been reviewed in detail. ${ }^{19}$

Focused particle beam induced etching of chromium and chromium oxide has been one of the most important industrial applications in the past years. For photomask repair the removal of excess of absorbing chromium is essential. Chlorine-assisted FEB induced etching proved difficult due the the missing selectivity of the etch process with respect to $\mathrm{CrO}_{x}$ and $\mathrm{Cr}$ layers and the underlying $\mathrm{SiO}_{2}$ material. Overetching into the $\mathrm{SiO}_{2}$ material would induce unwanted light intensity changes in the photomask due to interference effects. Adding nitrogen in the form of ammonia improved the selectivity, but the breakthrough of high selectivity was obtained by using $\mathrm{NO}_{2} \mathrm{Cl}$ or $\mathrm{NOCl}$ gas that selectively etches
$\mathrm{CrO}_{x}$ and $\mathrm{Cr}$, but seems not to attack at all $\mathrm{SiO}_{2}{ }^{398}$ The presence of oxygen and chlorine atoms in the dissociation products upon irradiation helps to form the volatile $\mathrm{CrO}_{2} \mathrm{Cl}_{2}$ compound, whereas the presence of nitrogen helps to protect against $\mathrm{SiO}_{2}$ etching by reacting locally with the $\mathrm{Si}$ to $\mathrm{Si}_{3} \mathrm{~N}_{4}$, a better etch resistant material. The high polarity of the precursor results in a sufficiently long residence time for inducing the chemical reaction. Table XX summarizes the FEB induced etching results.

\section{FIB gas enhanced etching}

The same precursor compounds as described for FEB induced etching above can often be used for the enhancement of physical sputtering in FIB (milling). Compounds employed so far are very similar or identical to plasma etching gases developed for microelectronics industry. Indeed gasassisted FIB can be regarded as a local version of plasma etching or reactive ion etching, although the ion energy is much higher for FIB. The difference between the precursors for FEB and FIB induced etching is that for FIB, the precursor gas can contain nonvolatile elements (which can be physically sputtered away). However, it is generally better to 
TABLE XX. Etch precursor gases, materials to be etched, etching conditions, and results for focused electron beam induced etching.

\begin{tabular}{|c|c|c|c|c|}
\hline Precursor gas & $\begin{array}{l}\text { Material to be } \\
\text { etched }\end{array}$ & Conditions & Results & Ref. \\
\hline $\begin{array}{l}\mathrm{XeF}_{2} 1.5 \mathrm{keV} \\
\text { electrons }\end{array}$ & $\mathrm{SiC}, \mathrm{SiO}_{2}, \mathrm{Si}_{3} \mathrm{~N}_{4}$ & $\begin{array}{l}\text { current, } 45 \mu \mathrm{A} \text {; current } \\
\text { density: } 50 \mathrm{~mA} / \mathrm{cm}^{2} ; 8 \times 10^{-2} \mathrm{~Pa} \\
\text { (also studied irradiation with } \\
450 \mathrm{eV} \mathrm{Ar}^{+} \text {) }\end{array}$ & $\begin{array}{l}\text { Etch rate: } \\
\mathrm{Si}_{3} \mathrm{~N}_{4}: 60 \mathrm{~nm} / \mathrm{min} \\
\mathrm{SiO}_{2}: 20 \mathrm{~nm} / \mathrm{min}\end{array}$ & 399 \\
\hline $\begin{array}{l}\mathrm{XeF}_{2} \\
0.7 \mathrm{keV} \\
\text { electrons }\end{array}$ & $\mathrm{Si}$ & $(1-2) \times 10^{-4}$ Torr; $30-60 \mu \mathrm{A} / \mathrm{cm}^{2}$ & $\begin{array}{l}\text { Etch rate } \\
\text { enhancement: } 2.2\end{array}$ & 309 \\
\hline $\begin{array}{l}\mathrm{XeF}_{2} \\
10 \mathrm{keV} \\
\text { electrons }\end{array}$ & $\mathrm{Si}$ & $\begin{array}{l}100 \mathrm{pA} ; 0.1 \mu \mathrm{m} \text { diameter, } \\
100 \mathrm{Si} \text { atoms per electron }\end{array}$ & $\begin{array}{l}\text { Etch rate } \\
\text { enhancement: } 2\end{array}$ & 287 \\
\hline $\begin{array}{l}\mathrm{XeF}_{2} \\
3,5,7,9 \text {, and } 12 \mathrm{keV} \\
\text { electrons }\end{array}$ & $\mathrm{SiO}_{2}$ & $\begin{array}{l}12.7 \mathrm{nA} \text {, substrate: } \mathrm{SiO}_{2} \text { on } \\
\mathrm{Si}\end{array}$ & $\begin{array}{l}\text { Etch rate: } \\
1-4 \mathrm{ML} / \mathrm{s}\end{array}$ & 400 \\
\hline $\begin{array}{l}\mathrm{H}_{2} \mathrm{O} \\
5 \mathrm{keV} \text { electrons }\end{array}$ & $\begin{array}{l}\text { Carbon from } \\
\text { contamination }\end{array}$ & $\begin{array}{l}71 \text { pA, substrate: InGaAs; } \\
90 \mathrm{~Pa} \mathrm{H}_{2} \mathrm{O}\end{array}$ & $\begin{array}{l}\text { Competition between } \\
\text { etching and } \\
\text { deposition takes place } \\
\text { as a function of } \\
\text { contamination level } \\
\text { and water pressure }\end{array}$ & 234 \\
\hline $\begin{array}{l}\mathrm{H}_{2} \mathrm{O} \\
25 \mathrm{keV} \\
\text { electrons }\end{array}$ & Diamond & $\begin{array}{l}300 \mathrm{pA} \text {, water flux at nozzle } \\
\text { exit } 2 \times 10^{18} / \mathrm{cm}^{2} \mathrm{~s} \text {. Electron flux, } \\
1 \times 10^{19} / \mathrm{cm}^{2} \mathrm{~s}\end{array}$ & Etch rate: $0.02 \mathrm{~nm} / \mathrm{s}$ & 384 \\
\hline $\begin{array}{l}\mathrm{O}_{2} \\
10 \mathrm{keV} \\
\text { electrons }\end{array}$ & Diamond & $\begin{array}{l}80 \mathrm{nA} ; 800 \mathrm{~nm} \text { beam } \\
\text { diameter; rectangle etching; } \\
\text { comparison of etch rates } \mathrm{O}_{2} \\
\text { and } \mathrm{H}_{2}\end{array}$ & $\begin{array}{l}\text { Oxygen etches faster } \\
\text { than hydrogen }\end{array}$ & 396 \\
\hline $\begin{array}{l}\mathrm{H}_{2} \\
10 \mathrm{keV} \text { electrons }\end{array}$ & Diamond & $\begin{array}{l}80 \mathrm{nA} ; 800 \mathrm{~nm} \text { beam } \\
\text { diameter; rectangle etching } \\
10 \mathrm{nA} ; 250 \mathrm{~nm} \text { beam } \\
\text { diameter, dot and line etching }\end{array}$ & $\begin{array}{l}\text { Using both gases, } \mathrm{O}_{2} \\
\text { and } \mathrm{H}_{2} \text {, no amorphous } \\
\text { carbon residue }\end{array}$ & 396 \\
\hline $\begin{array}{l}\mathrm{O}_{2} \\
2 \mathrm{keV} \text { electrons }\end{array}$ & $\begin{array}{l}\text { Carbon } \\
\text { contamination }\end{array}$ & $\begin{array}{l}18000 \mathrm{nA} \text {; beam diameter } \\
<0.5 \mathrm{~mm}, 10^{-4} \text { Torr } \mathrm{O}_{2}\end{array}$ & $\begin{array}{l}3.3 \mathrm{~nm} \mathrm{C} \text { etched in } \\
150 \mathrm{~min}\end{array}$ & 276 \\
\hline $\begin{array}{l}\mathrm{H}_{2} \\
10 \mathrm{eV}-1 \mathrm{keV} \\
\text { electrons }\end{array}$ & $\mathrm{Si}$ & $\begin{array}{l}\text { LE4 process; low electron } \\
\text { energy enhanced etching; } \\
2000 \mathrm{nA} ; \varnothing<1 \mathrm{~mm} \text {, } \\
P_{\text {res }}: 5 \times 10^{-11} \text { mbar }\end{array}$ & $\begin{array}{l}\text { Molecular } \mathrm{H}_{2} \text { is first } \\
\text { dissociated into } \mathrm{H} \\
\text { atoms in the gas } \\
\text { phase and desorbs } \\
\text { then under electron } \\
\text { irradiation } \mathrm{SiH}_{x} \text { units }\end{array}$ & 401 \\
\hline $\begin{array}{l}\mathrm{H}_{2} \\
1-15 \mathrm{eV} \\
\text { electrons }\end{array}$ & $\mathrm{GaN}$ & $\begin{array}{l}\text { LE4 process; electrons } \\
\text { impinge together with } \\
\text { molecular or atomic hydrogen } \\
\text { on heated substrates }\end{array}$ & $\begin{array}{l}\text { Very efficient etching } \\
\text { enhancement due to } \\
\text { electron irradiation }\end{array}$ & 402 \\
\hline $\begin{array}{l}\mathrm{H}_{2} / \mathrm{Cl}_{2} \\
\text { electrons }\end{array}$ & $\begin{array}{l}\mathrm{Si} \\
\mathrm{GaAs} \\
\mathrm{GaN}\end{array}$ & $\begin{array}{l}\text { LE4 process; a plasma } \\
\text { produces slow atoms that etch } \\
\text { in presence of electrons the } \\
\text { substrates }\end{array}$ & $\begin{array}{l}\text { Etch rate of } \mathrm{GaN} \text { with } \\
\mathrm{H}_{2}: 7-52.5 \mathrm{~nm} / \mathrm{min} \\
\text { for } 50-250{ }^{\circ} \mathrm{C}, \\
160 \mathrm{meV} \\
\text { activation energy }\end{array}$ & $\begin{array}{l}402 \\
\text { and references } \\
\text { cited therein }\end{array}$ \\
\hline $\begin{array}{l}\mathrm{WF}_{6} \\
\text { electrons }\end{array}$ & $\mathrm{SiO}_{2}$ & $\begin{array}{l}\text { Auger system, } 10 \mathrm{kV}, 8 \times 10^{-4} \mathrm{~A} / \mathrm{cm}^{2} \text {; } \\
2 \mathrm{C} / \mathrm{cm}^{2} \text {, } \\
\text { FEBID system: } 15 \mathrm{kV} ; 40 \mathrm{pA} \\
65^{\circ} \mathrm{C} ; 130{ }^{\circ} \mathrm{C}, 500 \mathrm{~nm} \mathrm{SiO} 2 \text { on } \\
\mathrm{Si}\end{array}$ & $\begin{array}{l}\text { W deposition } \\
\text { accompanies the } \mathrm{Si} \\
\text { etching }\end{array}$ & 287 \\
\hline $\begin{array}{l}\mathrm{Cl}_{2} \\
10 \mathrm{keV} \text { electrons }\end{array}$ & GaAs & $\begin{array}{l}100 \mathrm{pA} ; 0.1 \mu \mathrm{m} \text { diameter, } 0.6 \mu \mathrm{m} \\
\text { GaAs, }\end{array}$ & $\begin{array}{l}\text { GaAs etching, } \\
\text { electron enhancement } \\
\text { as compared to } 2 \mu \mathrm{m} \\
\text { without irradiation } \\
\text { at } 90^{\circ} \mathrm{C}\end{array}$ & 287 \\
\hline $\begin{array}{l}\mathrm{Cl}_{2} \\
2-3 \mathrm{keV} \\
\text { electrons }\end{array}$ & $\mathrm{CrO}_{x}$ & $\begin{array}{l}10 \mathrm{~mA} / \mathrm{cm}^{2} ; 0.1 \mu \mathrm{m} \text { diameter, } \\
\mathrm{mTorr} \text { range; } 10^{-5} \mathrm{Torr} ; 10 \mathrm{~nm} \\
\mathrm{CrO}_{x} \text { on } \mathrm{Si} \text { and } \mathrm{Pd} / \mathrm{Ag} \text { alloy }\end{array}$ & Unselective etching & $303,304,403$, and 404 \\
\hline $\begin{array}{l}\mathrm{ClF}_{3} \\
10 \mathrm{keV} \text { electrons }\end{array}$ & PMMA & $100 \mathrm{pA} ; 0.1 \mu \mathrm{m}$ diameter & PMMA etching & 287 \\
\hline
\end{tabular}


avoid any nonvolatile element (metals, carbon, etc.) in the precursor compound. The ideal etchant is thus composed of halogens, noble gases, nitrogen, oxygen, or hydrogen. Table XXI gives a summary of various gas enhancement factors. The experimental enhancement factors range within 0.2-75 for different materials systems. Factors $<1$ indicate, in fact, a passivation reaction of the molecules with the substrate atoms upon irradiation inhibiting the formation of volatile reaction products. For an overview of gas-assisted FIB etching of diamond, we refer to Ref. 153.

\section{E. Nonvolatile compounds}

Most compounds, containing more than three metal atoms even with the lowest polarizable ligands, such as $\mathrm{CO}$ or $\mathrm{PF}_{3}$ are barely volatile without decomposition. There are much more metal containing complexes existing and continuously synthesized such as uncharged molecules that are nonvolatile, and probably even more complexes that are charged and therefore tend to exist as ionic crystals. These low volatile compounds and films containing them can be altered by irradiation with charged particles such as ions and electrons. Recent approaches of synthesizing polymers containing metal clusters or nanoparticles in the polymer chain are a novel class of promising metal-rich compounds. The difference of clusters and nanoparticles adopted from Schmid ${ }^{419}$ is that clusters are monodispersed entities, which are precisely known and characterized by the same number of metal atoms in each unit, and nanoparticles are small particles (2-100 $\mathrm{nm}$ ) but with a size distribution. Prominent examples are the stabilized gold clusters obtained with the same type of ligands as the high purity gold resulting trifluorophosphine gold chloride compound. ${ }^{333}$ The full two shells around one gold atom 55 cluster stabilized with 12 triphenylphosphine and $6 \mathrm{Cl}$ ligands is one of the first examples. ${ }^{419}$

It is straightforward that layers of such compounds have also been irradiated with charged particles in order to change the properties. Partially altered material results in nanocomposites with strongly changing electrical properties. ${ }^{420}$ full decomposition should result even in pure metal films. The main advantage of this solid film approach is the potentially high processing speed because the needed precursor amount is applied before beam irradiation; no replenishment time limitation can occur.

Decomposition of solid layers composed of or containing metal organic precursors has been studied for a large number of metals in the past 20 years. The layers have been obtained by spraying, spin coating, dip coating, condensation from the gas phase, hydrothermal growth, self-assembly, or other methods. The present review article does not aim to cover the large number of papers that have been published in this direction. Successful decomposition with laser beams, ion beams, and electron beams has been reported for some compounds. Gold lines have been obtained by electron beam irradiation of commercially available gold containing inks. ${ }^{421}$ A focused $\mathrm{Ga}^{+}$beam of $20 \mathrm{keV}$ energy induced the decom- position of palladium acetate films and resulted in conductive lines. ${ }^{422}$ Inspired by this work one of the authors tried to improve the geometrical limitations and the electrical properties of the metal lines by irradiation of cluster compounds that should improve the quantity of metal in the layer and therefore decrease the shrinkage of the film during decomposition. The magic number gold cluster compound $\mathrm{Au}_{55}\left(\mathrm{PPh}_{3}\right)_{12} \mathrm{Cl}_{6}$ (Ref. 319) and self-synthesized iridium cluster compounds such as the tetraethylammonium salt of undecacarbonylobromotetrairidium $\left[\mathrm{Ir}_{4}(\mathrm{CO})_{11} \mathrm{Br}\right] \mathrm{NEt}_{4}$ (Ref. 319) have been irradiated with Ga ions and resulted in conductive lines. The difficulty of such approaches is the limited line thickness that can be obtained. Independent of the compound, generally shrinkage in thickness of more than $80 \%$ takes place and the deposited thickness is limited by the originally applied layer thickness. Another problem of this approach is the limited adhesion of the obtained deposits on the substrate. The limited purity of the deposits and the weak adhesion are arguments against using these kinds of processes, but the strongest argument against the decomposition of metal containing films for pure metal structures is the similarity to well established, much faster, and good quality of metal deposit resulting lift-off processes. In comparison to these processes, metal organic layer approaches can be considered as low efficiency lithography because the applied electron, ion, or photon doses to completely decompose the layer to pure metal are generally much higher than those needed in optimized resist solubility change due to exposure. On the other hand, a revival of the metal containing film irradiation arises on the horizon, which is the specific patterned film production of functional nanocomposites for optical, magnetic, electrical, mechanical, and thermal properties.

\section{PROCESS CONTROL AND CHARACTERIZATION OF DEPOSITS}

\section{A. Time-resolved process control}

\section{Reflectometry}

Real-time in situ reflectometry during FEB deposition was reported in Ref. 379. The setup is shown in Fig. 38(a), which basically consists of a monochromatic laser focused on the growing transparent or semitransparent deposit inside the SEM chamber and a readout of the optical signal. The principle is such that the intensity of the reflected laser beam depends on the optical thickness of the deposit and its optical absorption. A periodic signal is obtained, which allows calculating the real and imaginary parts of the refractive index at the laser wavelength from the period and the amplitude decay after having performed a thickness calibration. Comparing the optical thickness to the thickness measured by profilometry at various exposure times reveals if the deposited material is homogeneous or if changes in composition, i.e., the refractive index, occurred during growth. This kind of "tomography" information acquired in situ would replace time-consuming FIB cross section preparation. However, 
TABLE XXI. Gas-assisted FIB etching. The rate enhancement refers to milling without precursor gas.

\begin{tabular}{|c|c|c|c|c|}
\hline Substrate & $\begin{array}{l}\text { Ion } \\
\text { (energy, keV) }\end{array}$ & $\begin{array}{l}\text { Gas (pressure on } \\
\text { substrate or flux) }\end{array}$ & $\begin{array}{l}\text { Rate } \\
\text { enhancement }\end{array}$ & Reference \\
\hline $\mathrm{Si}$ & $\mathrm{Ga}(30)$ & $\begin{array}{l}\mathrm{Cl}_{2} \\
\text { (4 mTorr) }\end{array}$ & 20 & 405 \\
\hline $\mathrm{Si}$ & $\mathrm{Ga}(25)$ & $\begin{array}{l}\mathrm{H}_{2} \mathrm{O} \\
\text { (70 mTorr) }\end{array}$ & 0.3 & 137 \\
\hline $\mathrm{Si}(100)$ & $\begin{array}{l}\mathrm{Ga}(20) \\
\mathrm{Ga}(5)\end{array}$ & $\begin{array}{l}\mathrm{Cl}_{2} \\
\left(3 \times 10^{14} \text { molecules } / \mathrm{cm}^{2} \mathrm{~s}\right)\end{array}$ & $\begin{array}{l}39^{\mathrm{b}} \\
18^{\mathrm{b}}\end{array}$ & 406 \\
\hline $\mathrm{Si}(111)$ & $\mathrm{Ga}(30)$ & $\begin{array}{l}\mathrm{Cl}_{2} \\
\left(4 \times 10^{17} \text { molecules } / \mathrm{cm}^{2} \mathrm{~s}\right)\end{array}$ & $11.8-15.8^{\mathrm{c}}$ & 407 \\
\hline $\begin{array}{l}\mathrm{Si} \\
\text { (off axis) }\end{array}$ & & $\begin{array}{l}\mathrm{I}_{2} \\
\left(10^{15} \text { molecules } / \mathrm{cm}^{2} \mathrm{~s}\right)\end{array}$ & 11 & 408 \\
\hline GaAs (100) & $\mathrm{Ga}(30)$ & $\begin{array}{l}\mathrm{Cl}_{2} \\
\left(4 \times 10^{17} \text { molecules } / \mathrm{cm}^{2} \mathrm{~s}\right)\end{array}$ & $\begin{array}{l}10 \\
(14.8 \text { for } \\
\text { optimized } \\
\text { conditions })\end{array}$ & 407 \\
\hline $\operatorname{GaAs}(100)$ & $\begin{array}{l}\mathrm{Ga} \\
(0.2-15)\end{array}$ & $\begin{array}{l}\mathrm{Cl}_{2} \\
\left(3 \times 10^{18} \text { molecules } / \mathrm{cm}^{2} \mathrm{~s}\right)\end{array}$ & $\begin{array}{l}20-10 \\
\text { (for } 0.2 \text { and } \\
15 \mathrm{keV} \text { ions) }\end{array}$ & 409 \\
\hline InP & $\mathrm{Ga}(35)$ & $\begin{array}{l}\mathrm{Cl}_{2} \\
(1.3 \text { mTorr })\end{array}$ & $\begin{array}{l}20-30 \\
\left(140^{\circ} \mathrm{C}\right)\end{array}$ & 405 \\
\hline InP & $\mathrm{Ga}(30)$ & $\mathrm{I}_{2}$ & $\begin{array}{l}10(\mathrm{RT}) \\
66\left(200{ }^{\circ} \mathrm{C}\right)\end{array}$ & 410 and 411 \\
\hline $\mathrm{SiO}_{2}$ & $\operatorname{Ar}(50)$ & $\begin{array}{l}\mathrm{XeF}_{2} \\
(20 \text { mTorr })\end{array}$ & 100 & 69 \\
\hline $\mathrm{SiO}_{2}$ & $\mathrm{Ga}(20)$ & $\begin{array}{l}\mathrm{XeF}_{2} \\
\left(2 \times 10^{19} \text { molecules } / \mathrm{cm}^{2} \mathrm{~s}\right)\end{array}$ & 10 & 230 \\
\hline $\mathrm{SiO}_{2}$ & $\mathrm{Ga}(30)$ & $\begin{array}{l}\mathrm{XeF}_{2} \\
\left(10^{-5} \text { Torr in chamber }\right)\end{array}$ & 12 & 412 \\
\hline $\mathrm{Si}_{3} \mathrm{~N}_{4}$ & $\operatorname{Ar}(50)$ & $\begin{array}{l}\mathrm{XeF}_{2} \\
(20 \text { mTorr })\end{array}$ & 40 & 69 \\
\hline W & $\mathrm{Ga}(20)$ & $\begin{array}{l}\mathrm{XeF}_{2} \\
\text { (2 Torr) }\end{array}$ & $\begin{array}{l}\text { 15-75 } \\
\text { (depending on } \\
\text { dwell time) }\end{array}$ & 413 \\
\hline $\mathrm{Al}$ & & $\mathrm{Cl}_{2}$ & $5-10$ & 414 \\
\hline $\mathrm{Al}$ & $\mathrm{Ga}(30)$ & $\begin{array}{l}\mathrm{Cl}_{2} \\
\left(4 \times 10^{17} \text { molecules } / \mathrm{cm}^{2} \mathrm{~s}\right)\end{array}$ & $\begin{array}{l}\sim 3 \\
\text { (27 for } \\
\text { optimized } \\
\text { conditions) }\end{array}$ & 407 \\
\hline $\mathrm{Cu}$ & $\mathrm{Ga}(45)$ & $\begin{array}{l}\mathrm{Cl}_{2}+\mathrm{NH}_{3} \\
1: 1,0.1 \text { Torr }\end{array}$ & $6-12$ & 415 \\
\hline $\mathrm{Ti}$ & $\mathrm{Ga}(30)$ & $\begin{array}{l}\mathrm{XeF}_{2} \\
1.3 \times 10^{20} \text { (at needle } \\
\text { outlet) }\end{array}$ & 7 & 232 \\
\hline $\mathrm{Nb}$ & $\mathrm{Ga}(30$ ?) & $\mathrm{XeF}_{2}$ & 7.2 & 416 \\
\hline $\mathrm{Nb}$ & $\mathrm{Ga}(30 ?)$ & $\mathrm{I}_{2}$ & 8.8 & 416 \\
\hline PMMA & $\mathrm{Ga}(25)$ & $\begin{array}{l}\mathrm{H}_{2} \mathrm{O} \\
\text { (70 mTorr) }\end{array}$ & 20 & 137 \\
\hline PMMA & $\begin{array}{l}\mathrm{He}, \mathrm{Ne}, \mathrm{Ar}, \mathrm{Kr} \\
\mathrm{Xe}(50)\end{array}$ & $\begin{array}{l}\text { Mixtures of } \mathrm{XeF}_{2} / \mathrm{O}_{2} \\
(10-100 \text { mTorr })\end{array}$ & $1-5$ & 417 \\
\hline $\mathrm{C}$ (diamond) & $\mathrm{Ga}(25)$ & $\begin{array}{l}\mathrm{H}_{2} \mathrm{O} \\
\text { (70 mTorr) }\end{array}$ & 10 & 137 \\
\hline $\begin{array}{l}\mathrm{C} \text { (amorphous } \\
\text { and diamond) }\end{array}$ & $\mathrm{Ga}(50)$ & $\mathrm{XeF}_{2}, \mathrm{NH}_{3} / \mathrm{Cl}_{2}$ & $4-7$ & 154 \\
\hline $\mathrm{Si}, \mathrm{SiO}_{2}, \mathrm{Si}_{3} \mathrm{~N}_{4}$ & $\mathrm{Ga}(25)$ & $\begin{array}{l}\mathrm{H}_{2} \mathrm{O} \\
\text { (70 mTorr) }\end{array}$ & $\sim 0.3^{\mathrm{d}}$ & 137 \\
\hline $\mathrm{Cr}$ & $\mathrm{Ga}(50)$ & $\begin{array}{l}\mathrm{XeF}_{2}, \mathrm{O}_{2}, \mathrm{H}_{2} \mathrm{O}, \mathrm{Cl}_{2}, \mathrm{Br}_{2} \\
\mathrm{NH}_{3} / \mathrm{Br}_{2}, \mathrm{CO} / \mathrm{Br}_{2}\end{array}$ & $2\left(\mathrm{Br}_{2}\right)$ & 418 \\
\hline
\end{tabular}

${ }^{\mathrm{a}}$ The gas pressure values may be very uncertain. The measurement and/or estimation procedures are not specified in all cases.

${ }^{\mathrm{b}}$ These enhancement factor values (as well as others in the table, no doubt) depend strongly on the milling rate with no gas present. The authors quote 1.5 atoms/ion at both 5 and $20 \mathrm{keV}$ but mention large variations from run to run. This value is low compared to what is quoted in Table $\mathrm{V}$ above. The discrepancy may be due to some of the reasons we discussed in Sec. II C 2 on milling yield.

${ }^{c}$ The difference between the two results on silicon etching (Refs. 406 and 407) may be due to the fact that in reference g the current density in the Ga beam was five orders of magnitude lower (the beam was deliberately defocused) than the current density in Ref. 407 . So the results in Ref. 407 of the etch rate may have been influenced by a depletion of precursor molecules.

${ }^{\mathrm{d}}$ All three materials, $\mathrm{Si}, \mathrm{SiO}_{2}$, and $\mathrm{Si}_{3} \mathrm{~N}_{4}$, "etch" more slowly by about a factor of 3 than they mill with no gas present. Presumably this is due to the fact that the material reoxidizes as it is being milled. 
a)

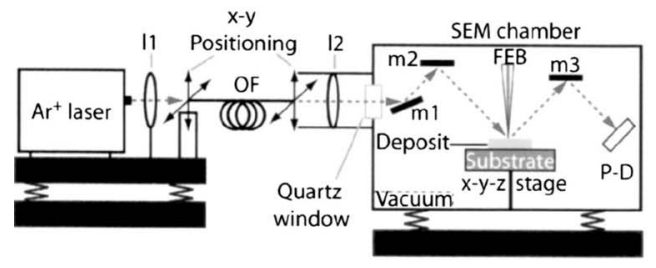

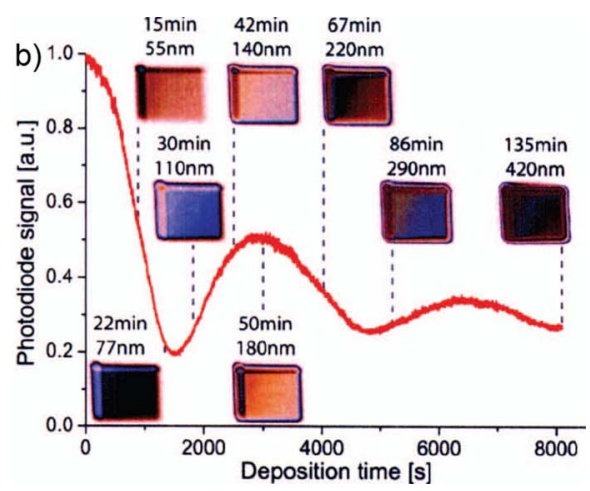

FIG. 38. (a) Simplified drawing of the reflectometry setup. Abbreviations stand for $\mathrm{m}=$ mirror, $1=$ lens, $\mathrm{OF}=$ optical fiber, and P-D=photodiode (from Ref. 379). (b) Reflectometry curves as a function of time for FEBID with $\mathrm{Si}\left(\mathrm{OCH}_{3}\right)_{4}$. Insets are optical microscope images of the deposits with corresponding deposition time and thickness (from Ref. 379).

thesize of the deposit must be at least somewhat larger than the focused laser beam.

\section{Monitoring of sample current and secondary electron signal}

End point detection during FIB milling can be carried out by monitoring the sample (stage) current or the secondary electron current or by imaging the milled area during the process. All of these methods depend on the fact that the secondary electron yield depends on the material, and so as one mills into a multilayer film material the current or the image will change as the milling reaches the boundary between films. This technique is limited to holes that do not have high-aspect ratio so that a significant fraction of the secondary electrons leave the surface. See Fig. 39 for a sample current result of milling through an $\mathrm{Al} / \mathrm{SiO}_{2}$,

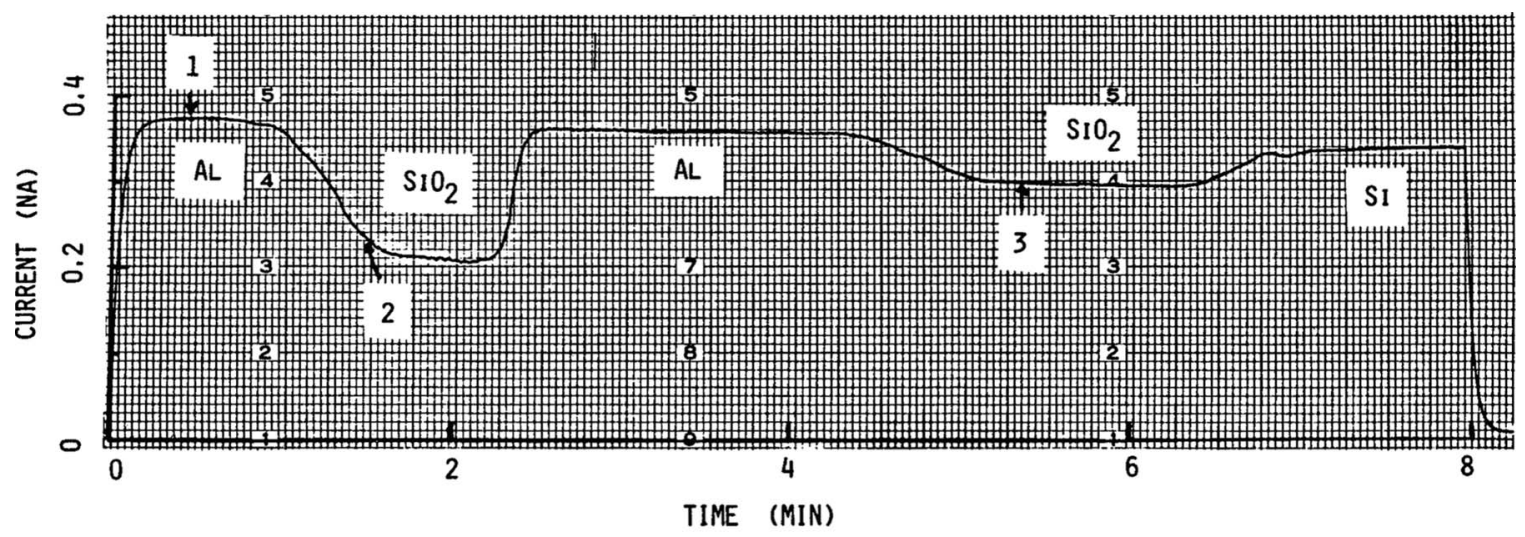

DISPLAY DURING MILLING

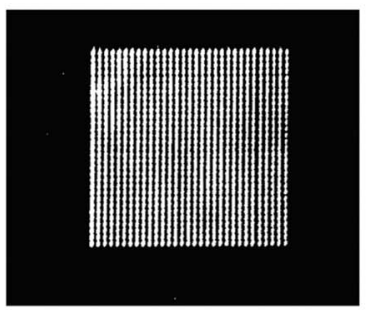

1

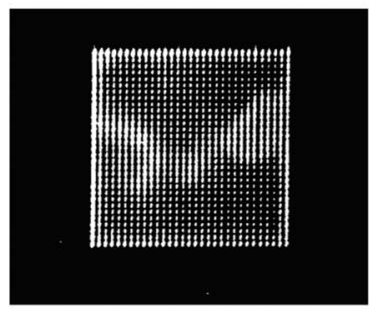

2

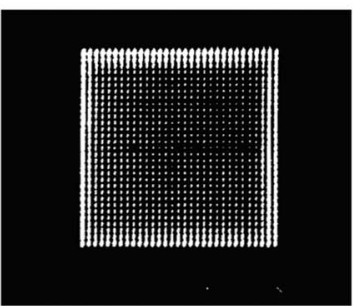

3

FIG. 39. Endpoint detection by time-resolved sample current measurements. The sample current is the difference of incident ion current and emitted electron (or ion) current. The emitted current is different for $\mathrm{SiO}_{2}$ and $\mathrm{Al}$ causing a variation as a function of milling time or via depth. The pit is $3 \times 3 \mu \mathrm{m}^{2}$. The lower part of the figure shows the display at three points (labeled on the curve) during milling. Note that because the pit being milled is becoming deeper, fewer secondary electrons escape at point 3 than at point 2 . At point 3 the milled pit is about $2 \mu \mathrm{m}$ deep. The Ga ion beam (68 keV) is stepped in $100 \mathrm{~nm}$ steps (from Ref. 157). 

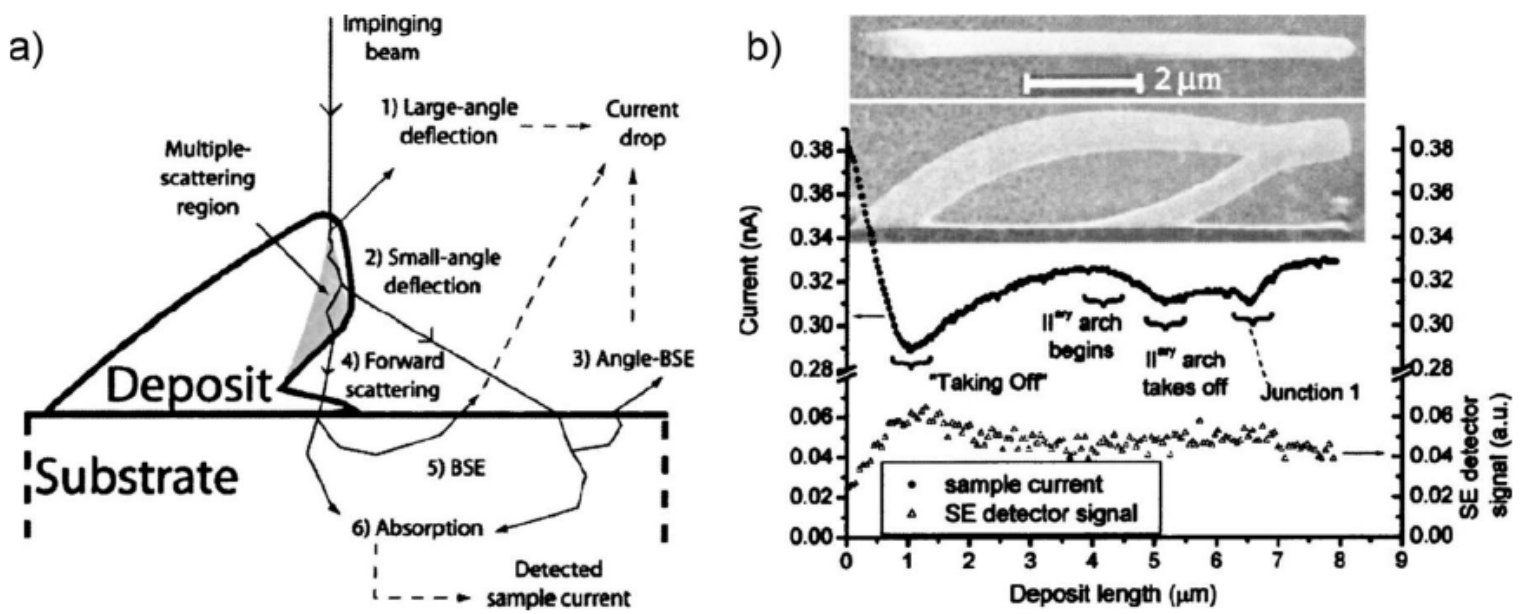

Fig. 40. (a) Schematic drawing of the electron paths in the deposits. The respective contributions of electrons trajectories to the measured current are highlighted (from Ref. 424). SEs generated at entrance and exit points of electrons are not shown. (b) In situ sample current and SE detector signal monitoring during deposition and correlation of the current features to the deposit geometry (top: top view; bottom: tilt view) (from Ref. 424).

$\mathrm{Al} / \mathrm{SiO}_{2}, \mathrm{Si}$ multilayer. ${ }^{157}$ Note that because $\mathrm{Al}$ is polycrystalline and the crystal grains mill at different rates, the transition from $\mathrm{Al}$ to $\mathrm{SiO}_{2}$ is not sharp. This is also seen in the image taken at the transition point.

Real-time monitoring of the sample current and the SE signal was applied and MC-simulated during FEB deposition of pillars, ${ }^{258,423}$ three dimensional periodic structures, ${ }^{424}$ and more complex three dimensional structures. ${ }^{336}$ The principle is shown in Fig. 40(a) from which it is seen that the sample current measures the balance of absorbed and exiting electrons in the sample. This signal offers for complicated three dimensional deposit geometries more sensitivity than the SE detector signal, as seen in Fig. 40(b). An ex situ calibration against geometry features is necessary to interpret the current-time curves properly. Once this precursor- and setupdependent calibration step (electron or ion beam acceleration, substrate, and deposit architecture) is performed, the current curve becomes a signature of the deposition process. This "fingerprint" is especially useful for in situ reproducibility checks, beam focus and astigmatism, or beam drifts.

A technique for end pointing the milling of bulk silicon from the backside of flip chip devices, using laser illumination to produce an optical beam induced current, is described in Ref. 425. The signal varies strongly with the thickness of the silicon above the bulk to well junction and can be used to reliably end point the bulk-well interface.

\section{Mass sensing}

Mass sensing during FEB or FIB deposition of large areas with quartz crystal microbalances was used since the 1960s for fundamental studies. ${ }^{110,188,236}$ Only recently, in situ realtime mass measurements during local FEB and FIB deposition and etching were reported. ${ }^{90}$ The principle is given in Fig. 41(a) and consists of a cantilever with piezoresistive readout being actuated by a piezoelectric actuator. The frequency response to mass changes must be calibrated due to the unknown cantilever force constant. ${ }^{426}$ From a typical measurement shown in Fig. 41(b), the adsorbed precursor
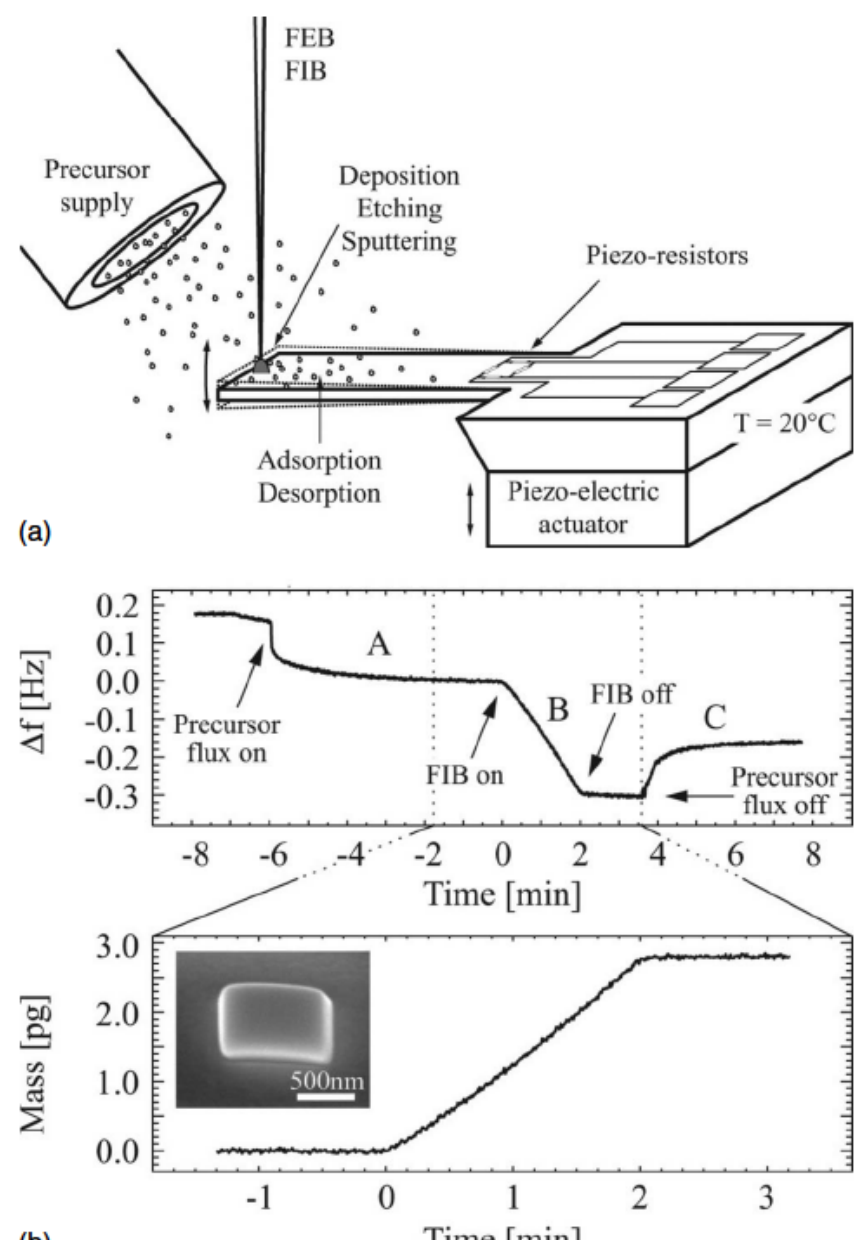

(b)

Time $[\mathrm{min}]$

FIg. 41. (a) Schematic diagram of the cantilever mass sensor for FIB/FEB induced process monitoring with local precursor supply from a microtube gas injection system. The mass added to or removed from the cantilever is detected as a negative or positive resonance frequency shift, respectively (from Ref. 90). (b) (Top) FIB induced deposition experiment from the precursor $\left(\mathrm{CH}_{3}\right)_{3} \mathrm{PtCpCH}$ : $\mathrm{A}=$ mass loading due to adsorption. $\mathrm{C}=$ mass loss due to desorption. $\mathrm{B}=\mathrm{FIB}$ exposure of a $1 \times 1 \mu \mathrm{m}^{2}$ rectangle. (Bottom) Evolution of the FIB $(30 \mathrm{kV}, 10 \mathrm{pA})$ deposited mass corresponding to part B. Inset: SEM tilt view $\left(52^{\circ}\right)$ of the FIB deposit (from Ref. 90). 

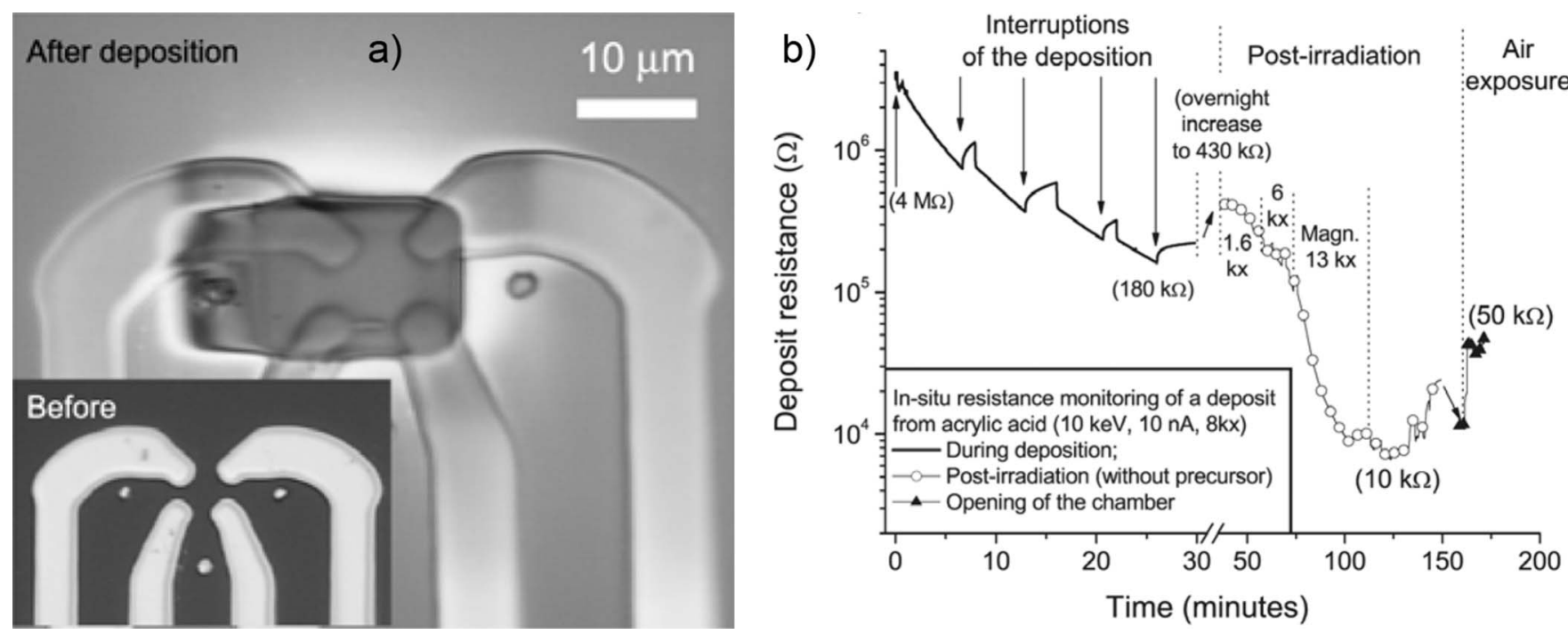

FIG. 42. (a) Four predeposited gold electrodes on $\mathrm{SiO}_{2} / \mathrm{Si}$ substrate (inset) connected by a rectangular FEB deposit from acrylic acid (from Ref. 269). The outer electrodes were used for a two point measurement. (b) Real-time two point resistance measurement corresponding to (a). Note that changes during interruptions, postirradiation, and air exposure can be monitored (from Ref. 269).

mass on the cantilever can be determined as well as the deposited (or etched) mass during FEB or FIB exposure.

Deposition or etch yields, in units of deposited or etched atoms per incident electron or ion, are precisely accessible according to the first part of Eq. (6.1)

$$
Y=\frac{N_{A} m / M}{I_{p} t / e_{0}}=R \frac{\rho}{M} N_{A} e_{0},
$$

where $I_{P} \cdot t$ is the incident dose during exposure time $t$ and beam current $I_{P}, m$ is the measured deposited or etched mass, and $M$ is the molar mass of the etched or deposited material. $N_{A}$ is the Avogadro constant and $e_{0}$ is the elementary charge. The molar mass of the etched material is generally known and etch yields can be derived in situ. For deposits the composition is generally unknown and the molar mass must be determined from composition measurements. Equation (6.1) represents a means to precisely determine yields since it does not require any assumption about the deposit density. In literature, frequently the deposition yields are calculated from deposition rates $R$ (here in units of volume per incident charge), assuming the density and composition of pure bulk material for the deposit. This has been shown incorrect. ${ }^{91}$ From mass measurements, the density $\rho_{\text {dep }}$ of the deposit can be obtained by measuring the deposit volume $V_{\text {dep }}$ and $\rho_{\text {dep }}$ $=m_{\text {dep }} / V_{\text {dep }}$. Finally, knowing the deposit density allows calculating the unknown specific volume of the deposited molecule entering into Eq. (2.1a) and (2.1b) via its molar mass: $V=M_{\text {dep }} / \rho_{\text {dep }}$. For precursors containing no hydrogen, the density can be estimated directly from the deposit composition. ${ }^{91}$

\section{In situ electrical resistance measurements}

Time-resolved resistance measurements during FEB deposition with $\mathrm{W}(\mathrm{CO})_{6}$ and acrylic acid were reported in Refs. 65 and 269. In situ measurements during FIB induced deposition with $\mathrm{W}(\mathrm{CO})_{6}$ are reported in Ref. 427. An ex- ample of an electrical resistance real-time measurement is shown in Fig. 42.

The in situ measurements permit to study the "electrical thickness" of the deposit. For constant length, width, and electrical resistivity, the resistance is inversely proportional to the deposited wire thickness. Hence, for a constant deposition rate an inverse relationship between the resistance and the deposition time is obtained. Any deviation from this relationship would point to changes affecting the charge carrier generation or transport and deposition kinetics such as charging effects or formation of intermediate species ${ }^{65,269}$ under irradiation during FEB and FIB processing. Also postirradiation and air exposure phenomena can be studied and related to eventual oxidation processes [see Fig. 42(b)]. Two point mesurements can be uniquely attributed to the deposit only if the contact resistance between electrodes and deposit is low compared to the deposit resistance. Alternatively, four point probe measurements would cancel contact resistance effects.

\section{In situ observation studies}

Dual or cross beam machines possessing a focused electron and ion beam allow in situ observation of the process. Information regarding the shape and process rate can be obtained in one experiment, which would otherwise necessitate a series of experiments stopped at differing intervals of the deposition or etch process. ${ }^{336}$ Due to its (almost) nondestructive interaction, generally, the focused electron beam is used for monitoring the FIB induced milling or deposition process. $^{428}$

Recently, in situ monitoring of the annular dark field signal during electron beam induced deposition on electron transparent membranes was used to feed back control the deposit's thickness homogeneity. ${ }^{429}$ This is especially useful for avoiding enhanced deposition on (predeposited) structures having a tilt to the incident electron beam (FEBID proximity effect). It was suggested that this method is ca- 

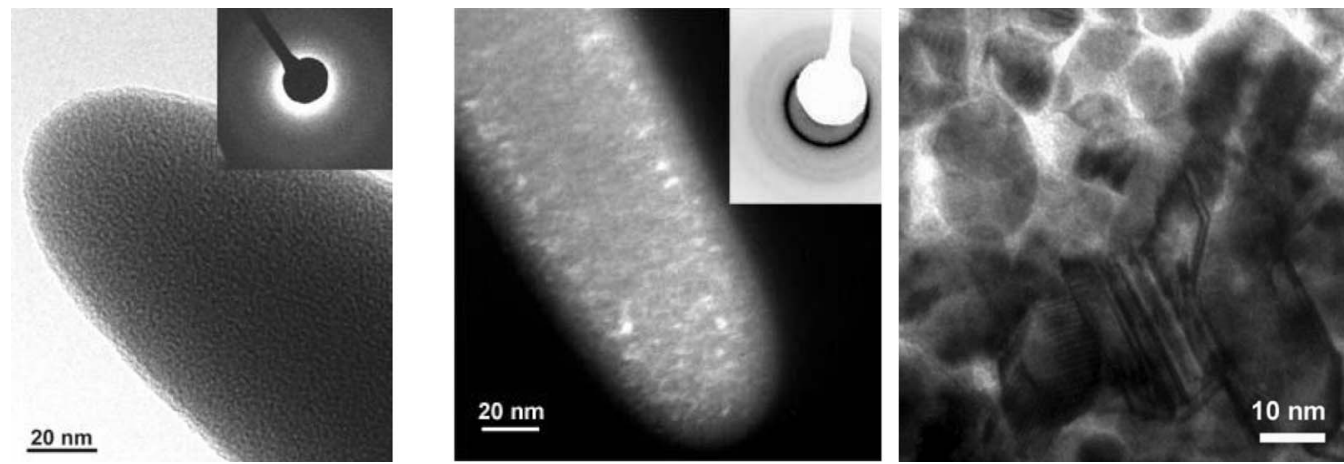

FIG. 43. TEM of FEB deposit substructures from differing precursors. Typical electron beam conditions were $25 \mathrm{keV}, 100 \mathrm{pA}$, and an electron flux of about $10^{19}$ electrons $/ \mathrm{cm}^{2} \mathrm{~s}$. (a) Amorphous dielectric $\mathrm{Si}-\mathrm{O}-\mathrm{C}$ tip from tetraethoxysilane (TEOS). (b) Nanocomposite tip (dark field TEM) from $\mathrm{Co}_{2}(\mathrm{CO})_{8}$. The

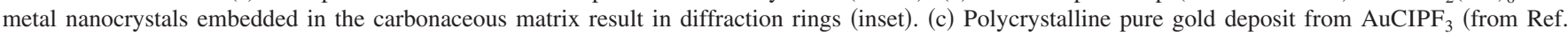
384).

pable of resolving the deposition of one single molecule, which would be the highest resolution of all the in situ techniques employed today.

\section{B. Composition and substructure}

The most commonly used methods comprise energy dispersive x-ray spectroscopy (EDXS), Raman spectroscopy, fast Fourier infrared (FTIR) spectroscopy, Auger electron spectroscopy (AES), and FIB cross section preparation. Although EDXS is well known we would like to point out that the standard quantification software of spectra often only allows for bulk samples. In other words, the excitation volume from which the x-rays escape should be as small as the deposit being probed. Estimations of this range can be found in Appendix A. A major limitation of EDXS is that hydrogen and the bonding states of carbon cannot be detected. FTIR measurements ${ }^{190}$ of FEB deposits obtained from organic precursors show that hydrogen is mainly bonded to $s p^{3} \mathrm{C}$ atoms, less to olefinic $\left(s p^{2}\right) \mathrm{C}$ atoms, and negligibly to aromatic $\mathrm{C}$ atoms in the deposit. Furthermore, the composition could be quantified to $\mathrm{C}_{9} \mathrm{H}_{2} \mathrm{O}$. Raman measurements allow quantification of the $s p^{3}$ and $s p^{2}$ contents in organic deposits ${ }^{190}$ or nanocomposite deposits containing a carbonaceous matrix. Depth composition profiling with AES in conjunction with ion sputtering may cause a postdecomposition of incompletely dissociated molecule fragments and may give, as a result, unreliable composition values. ${ }^{240}$

The internal structure of charged particle induced deposits can be generally classified into amorphous, nanocomposite (metal nanocrystals-carbon matrix), and polycrystalline (see Fig. 43 and 44). For FIB induced deposition similar internal structures can be observed and, additionally, gallium contamination (see Fig. 44).

Also composed crust-core structures were reported for FEB, when adding reactive gases ${ }^{388}$ or when beam heating effects $^{204}$ are involved. The physical properties of the deposits differ considerably from pure material (metal or dielectrics) when carbon from the precursor (or chamber) is incorporated. However, the resulting nanocomposite structure composed of metal nanocrystals embedded in a carbonaceous matrix proves in some cases very successful since it combines the functionality of the metal (electric and magnetic) with the mechanical stability and chemical protection of the carbonaceous matrix. Other applications require pure metal or dielectric deposits. For instance, the carbon content degrades optical transmissions or electrical conductivity at contacts.

The composition and microstructure of copper $^{376}$ (see Fig. 45) and gold ${ }^{430}$ films obtained by focused ion beam induced deposition using organometallic precursors have also been studied. In the case of gold the precursor was dimethylgold hexafluroacetylacetonate, and in the case of copper it was (hfa)CuVTMS [copper(I)-hexafluroacetylacetonate trimethylvinylsilane]. The films consist of either metal particles $50-100 \mathrm{~nm}$ diameter in a carbon matrix, or columns of

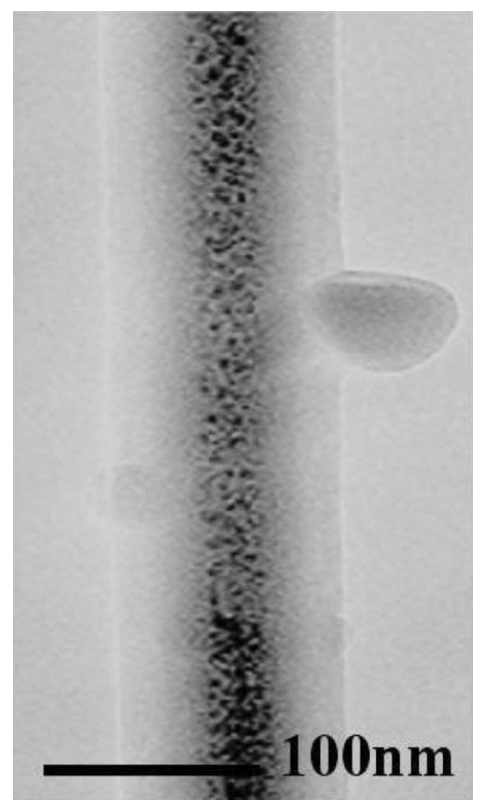

FIG. 44. TEM of an amorphous DLC pillar showing core $\mathrm{Ga}$ implantation (dark grains). Deposited with phenantrene and $30 \mathrm{keV} \mathrm{Ga}{ }^{+}$ions (taken from Ref. 27). 


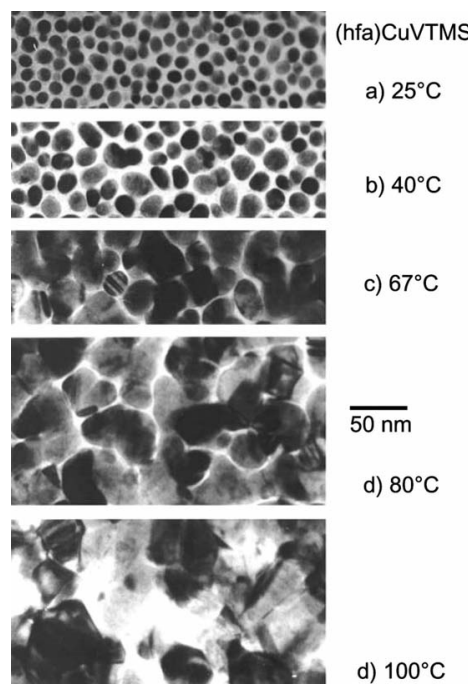

FIG. 45. Top view TEM micrographs of 50-100 nm thick copper containing films deposited at various temperatures using a $25 \mathrm{keV} \mathrm{Ga}^{+}$ion beam at an average current density of $20 \mu \mathrm{A} / \mathrm{cm}^{2}$. The local (hfa)CuVTMS gas pressure was $1.5 \mathrm{mTorr}$ and the ion beam dwell time was $100 \mathrm{~ns}$. The ion beam current was $60 \mathrm{pA}$ into a scanned area of $10 \times 30 \mu \mathrm{m}^{2}$ (Ref. 376). Chiang (Ref. 236) noted that thermal decomposition of the (hfa)CuVTMS molecule starts at $64{ }^{\circ} \mathrm{C}$

metal (similar diameters) oriented in the growth direction, or polycrystalline metal film with some carbon contamination. In general lower carbon content and lower resistivity films were obtained at lower ion beam current densities or higher substrate temperatures. Care should be taken that the global substrate temperature is below the temperature of thermal decomposition; otherwise the local resolution of the beam deposition is lost.

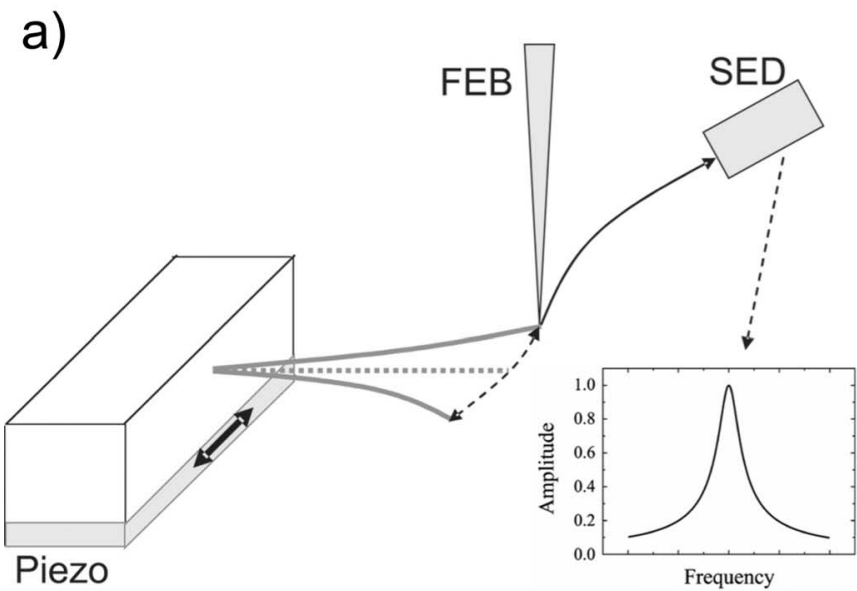

\section{SEM integrated mechanical measurements}

These measurements involve integration of nanomanipulation setups into the SEM. An overview on cantilever based force sensors and piezodriven vibration stages and their applications in scanning electron/ion microscopes can be found in Ref. 426. The determination of density and Young's modulus of FIB pillar deposits from phenanthrene and $\mathrm{W}(\mathrm{CO})_{6}$ was demonstrated in Refs. 431 and 432. They used a vibrational stage for resonance vibration of pillar deposits (see Fig. 46), and a second setup with a cantilever force sensor to bend the nanopillar. The mechanical properties as function of deposition parameters were investigated and showed a dependency with the flux ratio of precursor molecules and incident ions. Tensile strengths in the order of $1 \mathrm{GPa}$ were measured for FEB deposits from $\mathrm{Co}_{2}(\mathrm{CO})_{8}$ and $\mathrm{W}(\mathrm{CO})_{6} \cdot{ }^{433}$ SEM integrated atomic force microscopy measurements were reported in Refs. 426 and 434 and are very useful for topography information not accessible from the projected view in a scanning electron or ion microscope.

\section{APPLICATION FIELDS IN RESEARCH AND INDUSTRY}

The preceding discussion has shown that FEB and FIB processing is highly flexible for achieving nanoscale stuctures by using both material addition and material removal. Obviously, with any new precursor molecule more functional structures can be deposited or etched with tailored dielectric, electrical, mechanical, and optical properties. Presently, three industrial applications can be identified for FEB: photomask repair of the $65 \mathrm{~nm}$ node and below, high-aspect ratio diamondlike atomic force microscopy (AFM) sensors, and circuit editing of multilevel architectures. For FIB the most common industrial applications are TEM lamella prepara-
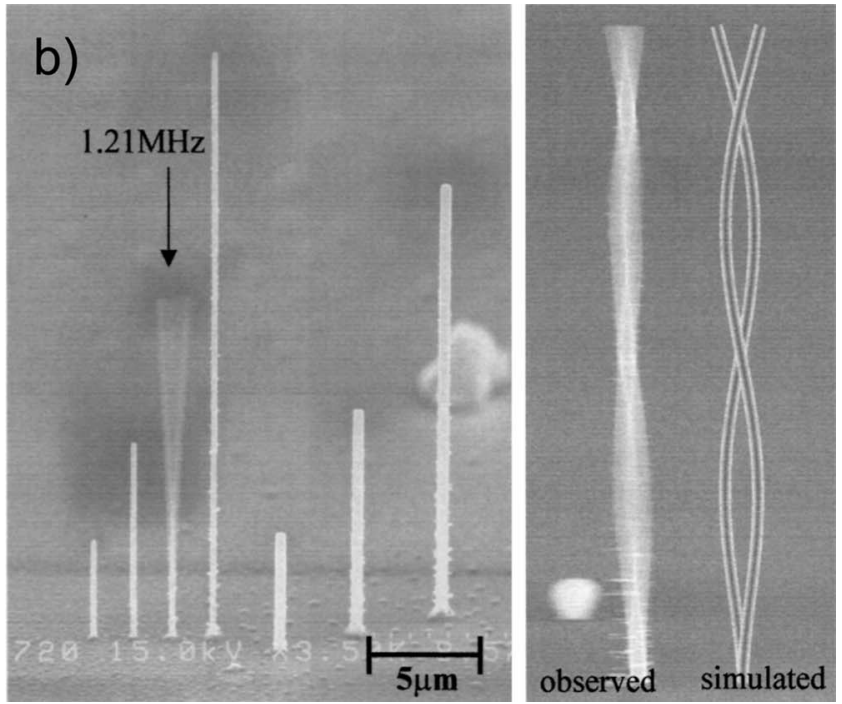

FIG. 46. (a) Principle of resonance experiment. When the resonance frequency of the pillar is approached, the secondary electron detector (SED) measures the peak curve since the pillar vibration intersects the focused electron beam (FEB) from (Ref. 426). (b) Tilt view of FIB deposited carbon pillars from phenanthrene precursor. Excitation of the fundamental resonance mode (left) and the second order resonance mode (right) from (Ref. 431). 

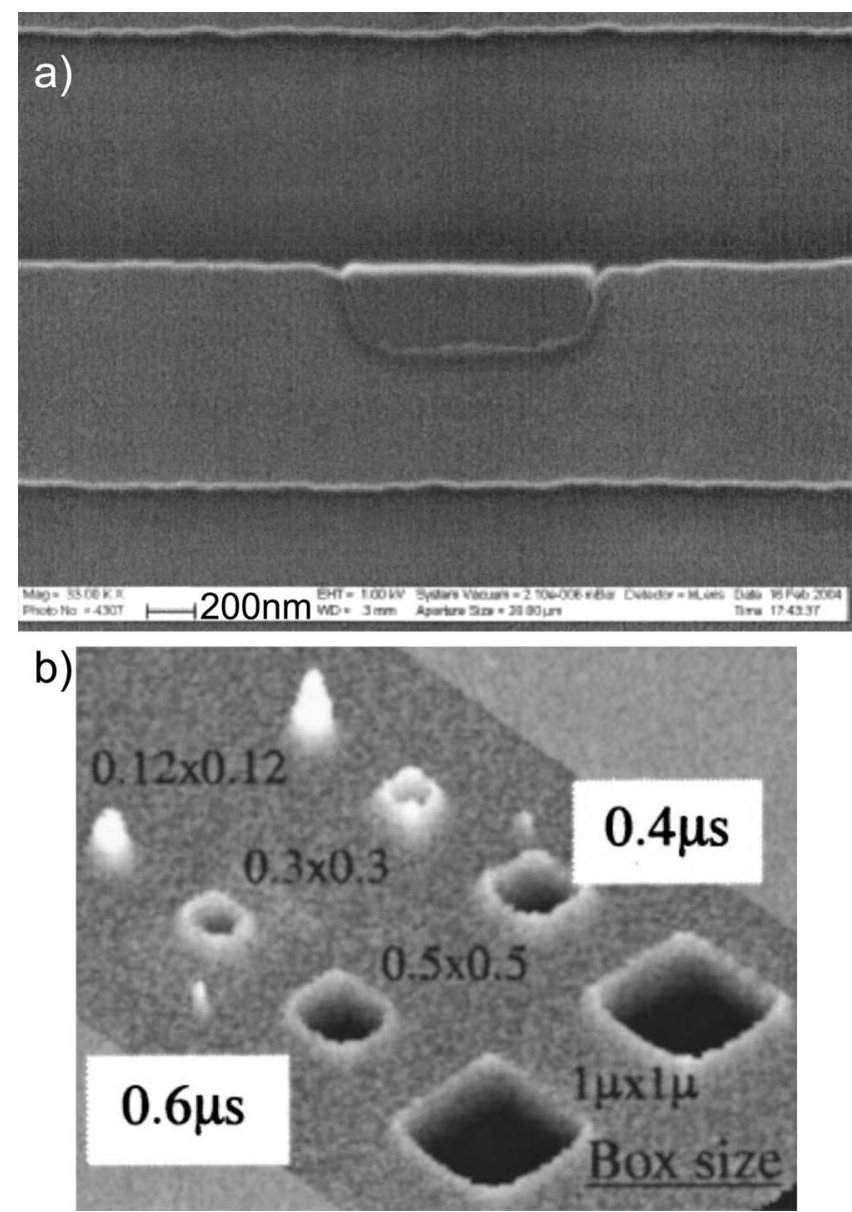

FIG. 47. High resolution FEB and FIB photomask repair. (a) SEM image of a FEB repaired mouse-bite-type clear mask failure using an organometallic Pt precursor and $1 \mathrm{keV}$ electrons at 10-250 pA (from Ref. 18). (b) AFM image of gas assisted FIB etching. Boxes were etched into TiN using $\mathrm{Br}_{2}$ gas and $\mathrm{Ga}^{+}$ions $(20 \mathrm{keV}, 22 \mathrm{pA})$ (from Ref. 435).

tion, tomography (e.g., failure analysis in integrated circuits), integrated circuit rewiring in the prototype stage, and write head trimming.

In this section we attempt to give a compact overview of the variety of FEB and FIB fabricated devices and industrial applications with a main focus on gas-assisted processing. Activities in device prototyping were and are increasingly numerous so that this list may be incomplete.

\section{A. Repair of photomasks}

Repair of defects in masks for extreme ultraviolet and advanced phase shift masks requires to deposit opaque or transparent material or to remove photomask material with high selectivity and without damage to the underlying material (Fig. 47). This application field was formerly a FIB domain, ${ }^{435}$ but due to the degradation of optical properties by $\mathrm{Ga}$ implantation and introduction of defects, the field increasingly becomes a FEB domain. ${ }^{17,436}$ FIB-related issues such as staining of quartz, overetching into sublayers, halos, layer intermixing, and riverbedding are avoided. ${ }^{18,437}$
A commercial tool for mask repair is sold since 2003 by Nawotec (now Zeiss) ${ }^{438}$ for the $65 \mathrm{~nm}$ node and below. Ongoing research in this field is mainly related to new precursor chemistries with high etch rate and selectivity and the avoidance of electrical charge buildup on insulating quartz masks. Competition in this field is high and FEB precursor chemistries are rarely published. Pure $\mathrm{SiO}_{2}$ FEB deposits using silane precursors together with oxygen as additional gas were reported. ${ }^{439}$ Optical measurements at $248 \mathrm{~nm}$ showed a 50\% transmission loss, which might be due to internal deposit inhomogeneity. FEB deposition of $\mathrm{SiO}_{2}$ obtained from TMOS and TMS by adding oxygen as reactive gas is reported. ${ }^{352}$ Optical transmission at $193 \mathrm{~nm}$ wavelength was measured to $80 \%$ and $99 \%$, respectively. The latter value fulfills the photomask repair requirements. Using the hydrogen-free tetraisocyanatosilane $\mathrm{Si}(\mathrm{NCO})_{4}$ molecule together with additional oxygen, light absorption at $193 \mathrm{~nm}$ wavelength was not detectable in $100 \mathrm{~nm}$ FEB deposited $\mathrm{SiO}_{2}{ }^{353}$ The irradiation induced reaction paths were investigated in detail. ${ }^{393}$

Other scientific studies comprise a comprehensive comparison of mask repair technologies including FIB, FEB, SPM, and laser. ${ }^{440}$ Without revealing the precursor compounds Edinger et al. ${ }^{441}$ reported on FEB $(1 \mathrm{keV}) \mathrm{MoSi}$ etching with an etch time of 4 min for a $70 \mathrm{~nm}$ deep and $1 \mu \mathrm{m}^{2}$ large box, a selectivity to quartz of $3: 1$, and a depth control of $3 \mathrm{~nm}$; FEB Cr etching with $11 \mathrm{~nm}$ resolution on phase mask; almost vertical $\mathrm{FEB} \mathrm{SiC}$ etching through a $300 \mathrm{~nm}$ thick membrane with about $40 \mathrm{~nm}$ linewidth; FEB TaN etching with $38 \mathrm{~nm}$ linewidth; and $\mathrm{Pt} / \mathrm{C}$ deposits with $4 \mathrm{~nm}$ critical dimension control. Liang et al. ${ }^{440}$ reported on FEB Pt/C deposition with $27 \mathrm{~nm}$ linewidth and $100 \mathrm{~nm}$ line thickness using $\mathrm{MeCpPt}(\mathrm{Me})_{3}$; FEB TaBN etching with a linewidth of $11 \mathrm{~nm}$ in a $70 \mathrm{~nm}$ film and a selectivity of 30:1; and FEB quartz etching with a linewidth of $25 \mathrm{~nm}$ in $100 \mathrm{~nm}$ depth and a depth control better than $5 \mathrm{~nm}$ using $\mathrm{XeF}_{2}$. Jinhua et al. ${ }^{442}$ reported a five times higher FEB etch rate for $\mathrm{Cr}$ with $\mathrm{XeF}_{2}$ using $10 \mathrm{keV}$ instead of $20 \mathrm{keV}$ electrons. Further details on $\mathrm{Cr}$ etching with FEB and FIB can be found in Tables XX and XXI, respectively. Gallium-FIB etching of $\mathrm{Cr}$ with the precursor mixtures $\mathrm{Br}_{2} / \mathrm{NH}_{3}, \mathrm{Br}_{2} / \mathrm{CO}_{2}$, and $\mathrm{Br}_{2}$ was studied in Ref. 418 and optical transmission rates found to range between $60 \%$ and $95 \%$ at $193 \mathrm{~nm}$.

\section{B. Scanning probe sensors}

Commercial cantilevers with ultrasharp high-aspect-ratio tips are used in AFM configuration to sense a variety of properties with nanometer resolution, such as topography, magnetic polarization, ferroelectric domains, and temperature. High resolution, high-aspect-ratio tips can be made by FEB induced deposition in a simple single step process ${ }^{256,444}$ by focusing the primary beam on the cantilever and depositing a pillar of the desired material (Fig. 48).

Mechanically resistant, high-aspect-ratio, high resolution AFM tips of diamondlike carbon are a commercial product from Nanotools. ${ }^{445}$ The mechanical properties are close 

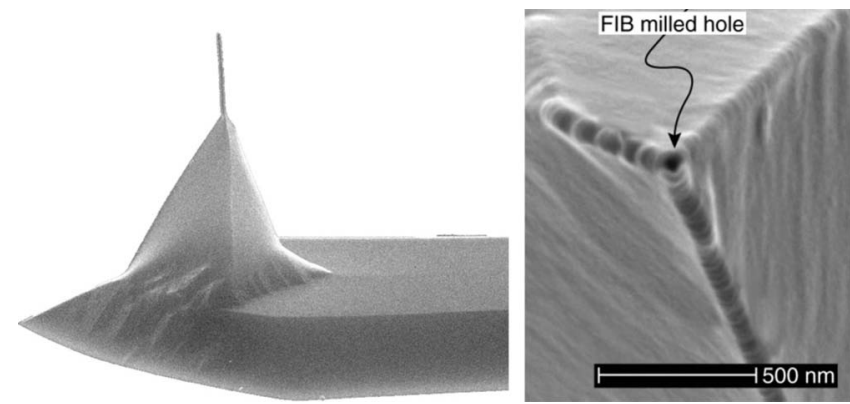

FIG. 48. High resolution FEB and FIB functionalized sensors. (a) FEB deposited MFM tip on commercial silicon AFM sensor. (b) A $40 \mathrm{~nm}$ diameter FIB milled hole into a magnetic coating of commercial silicon sensor (taken from Ref. 443).

to diamond; the elastic modulus is $0.5 \mathrm{TPa}^{446}$ Their use as a mechanical high resolution patterning tool was demonstrated. ${ }^{447}$

Scientific studies on FEB tip deposition investigated the role of electron beam energy, ${ }^{211,239,423}$ precursor pressure, ${ }^{448}$ secondary electron emission, ${ }^{61}$ different precursors, ${ }^{258}$ and electron beam size. ${ }^{321}$ As extensively discussed in the previous sections on modeling, it should be kept in mind that the tip apex resolution depends on the balance of precursor replenishment and depletion for a given beam size. This balance is very difficult to quantify experimentally. Experimental work concerning ultimate resolution of freestanding rod deposits by FEB can be found in Refs. 287 and 449-452.

The potential of automated FEB processing of cantilever wafers (image recognition, positioning, and autofocus) was investigated in Ref. 453. Apart from commercialized hard, high-aspect ratio, and sharp AFM tips, several functional scanning probe sensors were fabricated and analyzed at laboratory scale: magnetic FEB tips were characterized in Refs. 322 and 324, and magnetic properties of FEB structures measured. ${ }^{454,455}$ Optical fibers for scanning near field microscopy were functionalized with FEB deposited plasmonic gold structures ${ }^{456}$ and carbon waveguides. ${ }^{457}$ Scanning thermal sensors were shown in Refs. 458 and 459. FEB and FIB deposited silicon oxide nanostructures were used to reduce fluorescence quenching in apertureless scanning near field optical microscopy and create surface plasmon resonant tips, which avoids far field illumination and related background noise signals. ${ }^{460}$ The sidewall roughness with a specially FEB designed AFM tip was measured in Ref. 461. FIB deposition of tips suffers from comparatively "bad" resolution. The physical sputtering inherent to FIB is predominantly used in trimming of sensors, such as recording heads in magnetic harddisk storage, ${ }^{462-464}$ AFM sensors, ${ }^{443,465}$ or nanodispensers. ${ }^{466}$

\section{Circuit editing}

Focused ion beams, and in some cases also electron beams, are used to rewire integrated circuits in the prototyping phase. If an integrated circuit does not operate as expected after the initial design and fabrication, it can be altered and retested or diagnosed with these techniques.
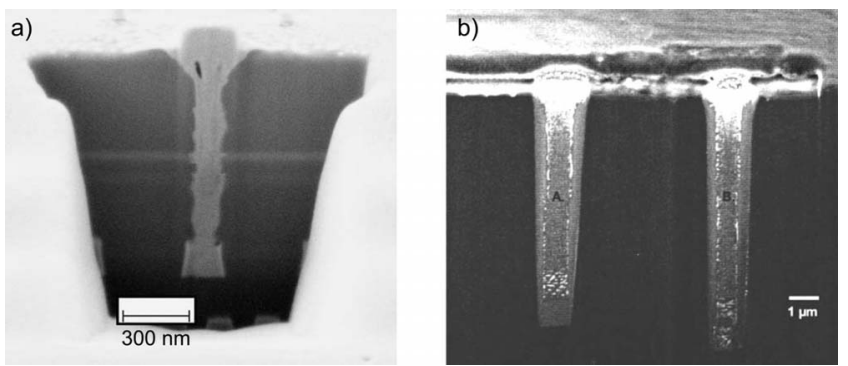

FIG. 49. (a) Cross section of a gas-assisted FEB processed via hole penetrating through several dielectric layers to a metal layer. The aspect ratio is 10 (depth: $1 \mu \mathrm{m}$; diameter: $100 \mathrm{~nm}$ ). After via hole etch the metal layer was connected by gas-assisted FEB metal deposition (courtesy of Nawotec/Carl Zeiss SMT). (b) FIB image of cross-sectioned FIB-Cu filled via holes using the precursor (hfa)Cu-VTMS. Left: $1 \times 1 \times 8.2 \mu \mathrm{m}^{3}$ via (may be incomplete cross section). Right: $1 \times 1 \times 9.2 \mu \mathrm{m}^{3}$ via (from Ref. 377 ).

Connections can be cut or made. These processes can be quite challenging in integrated circuits with multilevels of metallization. Alternatively vias can be etched (see Figs. 49 and 50) and metal pads can be deposited and connected to various points in the wiring so that the circuit can be tested with external probes. The technological challenge is to etch high-aspect-ratio holes through different layer materials at high resolution and to stop the process at the desired metal layer. Here end point detection methods as discussed in Sec. VI are in use. The other challenge is to connect the "buried" metal layer with other metal layers or top layer electronics by depositing an electrically conductive pillar inside the via hole.

A particularly challenging situation occurs when a connection needs to be made to an underlying metal layer, which is covered by metal layers above it. This requires deposition of both a metal and an insulator as illustrated in Fig. $50{ }^{380}$

The FEB repair of a transistor gate on GaAs using $\mathrm{W}(\mathrm{CO})_{6}$ was demonstrated. ${ }^{65}$ The deposit formed a Schottky barrier to the underlying GaAs substrate with an ideality factor of 1.4. Ion beam deposited contacts using $\mathrm{W}(\mathrm{CO})_{6}$ and $\mathrm{Ar}^{+}$and $\mathrm{H}_{2}^{+}$ions showed Ohmic behavior at $2 \mathrm{keV}$ incident energy due to ion induced damage and Schottky behavior at $0.5 \mathrm{keV}^{467}$

\section{Nanophotonics}

Nanophotonic structures realized by FEB and FIB comprise mainly two dimensional photonic crystals and plasmonic resonance structures in metal membranes. An overview on FEB and FIB deposition and etching for sub-100-nm fabrication of nanophotonic structures is given in Ref. 384 . Photonic crystals consist of an array of diffractive cylinders either realized by deposition of highly refractive material or by drilling holes into highly refractive material, namely, semiconductors. In both cases the refractive index change is against air. The scientific challenges for photonic band gap structure fabrication are (a) to deposit high-refractive index material with low absorption coefficient, (b) to deposit or etch high-aspect-ratio cylinders with length $\approx 3 \lambda$, and diameters $\approx(0.1-0.3) \lambda$ and pitches $\approx \lambda$ depending on the refrac- 


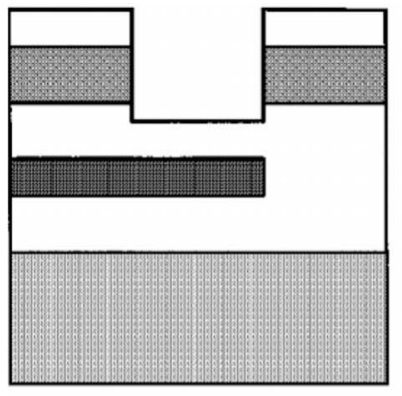

(a)

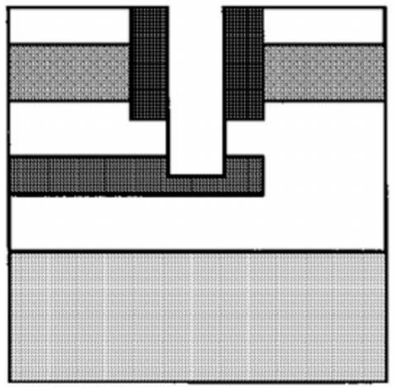

(c)

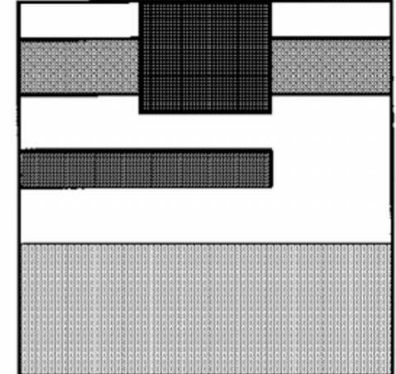

(b)

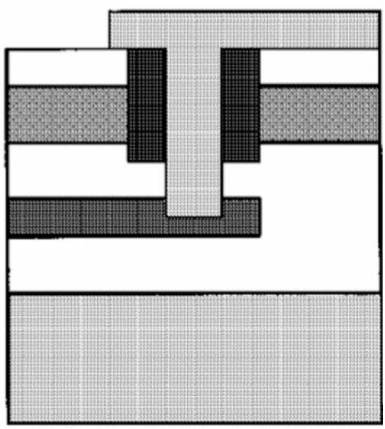

(d)

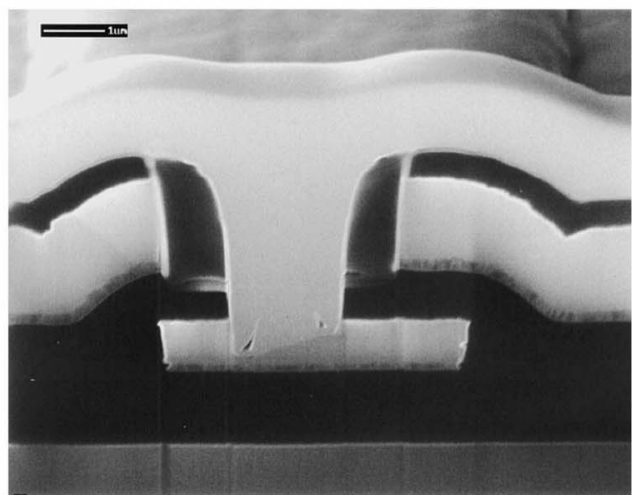

FIG. 50. Schematic of the fabrication sequence for making a connection to an underlying metallic layer covered by another layer. (a) Cut through the upper metal layer, (b) deposition of silicon dioxide insulator, (c) cutting of new hole down to lower metal layer, and (d) deposition of metal connection (from Ref. 380). (e) Focused ion beam image of the completed structure fabricated as shown in (d) (from Ref. 380). tive index, and (c) to deposit or etch with low surface roughness $<2 \mathrm{~nm}$ and constant diameter cylinder geometry. For plasmonic structures the metal purity is most important to keep damping losses low. The high addressability with FEB and FIB gives large flexibility in PBG and plasmonic structure design.

Refractive index measurements of FEB deposits from the precursors $\mathrm{Ti}$ tetraisopropoxide (TTIP) and TEOS in the spectral range from 300 to $900 \mathrm{~nm}$ are reported in Refs. 384 and 470. Using the precursors $\mathrm{Ti}\left(\mathrm{NO}_{3}\right)_{4}$ and TMOS, complex refractive indices of $n_{\mathrm{TiO}_{2}}=2.19+i 0.013$ at $514 \mathrm{~nm}$ and $n_{\mathrm{SiO}_{2} \mathrm{C}_{x}}=1.56+i 0.14$ at $514 \mathrm{~nm}$ were reported in Ref. 379 .

A FEB fabricated band gap filter in the 1.25-1.65 $\mu \mathrm{m}$ wavelength region using a Pt precursor was reported in Ref. 468 [see Fig. 51(a)]. The plasmonic resonance of a $60 \mathrm{~nm}$ gold FEB deposit from $\mathrm{Me}_{2} \mathrm{Au}$ (tfa) and on top of a SNOM tip was shown to homogenize and to enhance its light field. $^{456}$ FEB deposition of gold/carbon dot arrays from $\mathrm{Me}_{2}-\mathrm{Au}(\mathrm{acac})$ precursor was also reported. ${ }^{471}$ After ex situ annealing at $400{ }^{\circ} \mathrm{C}$ for $30 \mathrm{~min}$ in air, the dot arrays showed support of localized plasmon resonances. Figure 51(b) shows an example of FIB iodine-enhanced etching. A cylindrical hole is etched into InP to form a specific photonic band gap structure in a Y splitter. ${ }^{410}$ Special care was taken to prevent the InP from stray ions from the beam skirts by depositing a protective $\mathrm{W}$ or Pt layer (which was removed after local FIB etching). The addition of iodine and the use of a heat stage allowed for straight cylindrical holes with an aspect ratio of $>10$ and a surface roughness of $12.6 \mathrm{~nm}$ (root mean square), which is comparable to results obtained so far only by conventional dry etching methods.

Plasmonic resonance structures in metallic membranes fabricated by FIB milling are reported [Fig. 51(c)]. As a result of the coupling between the surface plasmons in metal structures and light waves, various interesting optical phenomena have been observed. Because of their lower plasmon frequencies, gold and silver are frequently used. These two metals are difficult to pattern at nanometer dimensions except by focused ion beam milling. For example, extraordinary optical transmission is observed through films of silver that have arrays of holes milled in them where the period of
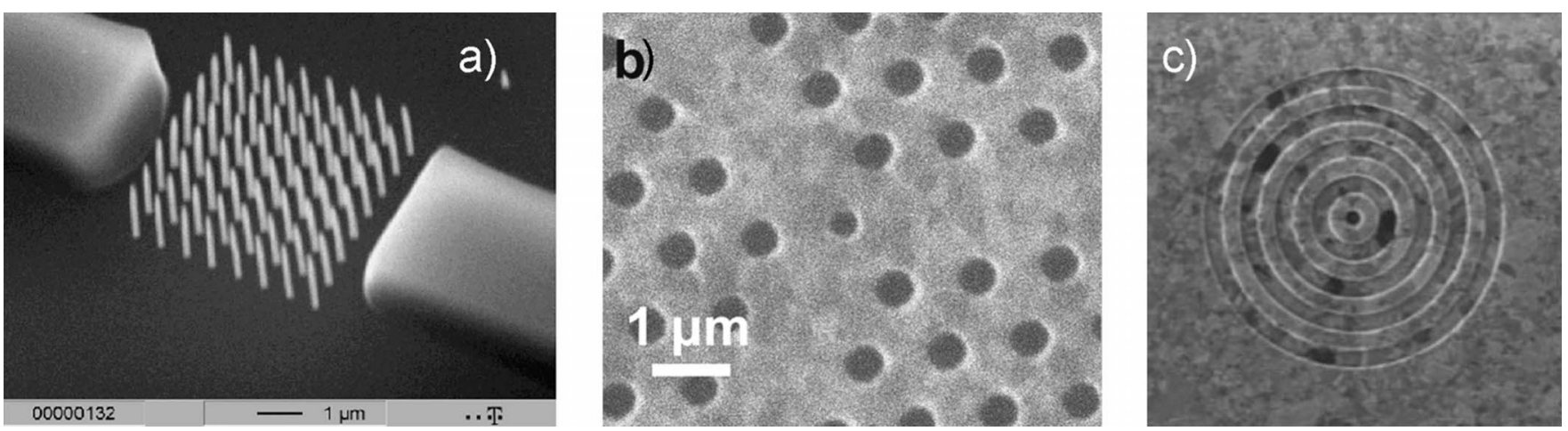

FIG. 51. FEB and FIB fabricated photonic crystals. (a) FEB deposited band gap filter (Ref. 468). (b) FIB (Ga ${ }^{+}$) iodine-enhanced etching of InP. The central hole was FIB etched into a photonic crystal structure (Y splitter) (from Ref. 410). (c) A FIB milled plasmonic resonance structure in a 300 nm thick silver membrane; the groove periodicity is $500 \mathrm{~nm}$, groove depth is $60 \mathrm{~nm}$, and hole diameter is $250 \mathrm{~nm}$ (Ref. 469 ). 
the array is less than the wavelength of the light. ${ }^{472}$ Another surprising effect was observed when a $300 \mathrm{~nm}$ thick silver membrane had a $250 \mathrm{~nm}$ hole focused ion beam milled through it and surrounded on both front and back by a concentric ring grating. When illuminated at normal incidence the transmission through this hole is totally unexpected. The transmitted light is forward collimated, wavelength dependent, and far more intense than would be expected for such a small hole. ${ }^{469}$ FIB milling was also used to fabricate specific aperture geometries into a $50 \mathrm{~nm}$ thick gold layer on top of an optical fiber ${ }^{473}$ and to fabricate Fresnel lenses at the front end of glass fibers and photonic band gap structures in a $\mathrm{LiNbO}_{3}$ waveguide. ${ }^{474,475}$

\section{E. Micro- and nanoelectronics}

FEB and FIB are used in this field as a versatile threedimensional single step fabrication, which does not require multistep processing as needed in e-beam lithography. There are a large number of laboratory demonstrators of electric circuit "LEGO" units such as resistors, wires, bonds, transistors, and sensors. Furthermore, failure inspection can be followed by a "just in place" repair or modification of electrical units can be performed very locally, for instance, in nanoelectromechanical system devices.

\section{Insulators and resistors}

Requirements for insulators are high resistance, high breakthrough voltage fields, and low intrinsic charges. Insulator deposition by FIB was reported for different silane precursors. ${ }^{382,394}$ For instance, with pentamethyl cyclopentasiloxane the best resistivity of $\rho_{\text {FIB }}=8 \times 10^{11} \Omega \mathrm{cm}$ and break down fields of $650 \mathrm{~V} / \mu \mathrm{m}$ were achieved. ${ }^{382}$ A comparison between FIB and FEB deposited insulators using TMOS showed $\rho_{\text {FIB }}=1 \times 10^{3} \Omega \mathrm{cm}$ and $\rho_{\text {FEB }}=1 \times 10^{6} \Omega \mathrm{cm}^{231}$ The "low" FIB deposit resistance is attributed to Ga contamination. A resistivity $\rho_{\mathrm{FEB}}=6 \times 10^{13} \Omega \mathrm{cm}$ and a leakage current of $2 \times 10^{-13} \mathrm{~A}$ is reported using an organosilicon precursor with additional $\mathrm{O}_{2}$ reactive gas supply for FEB. ${ }^{63}$ The addition of oxygen during deposition removes codeposited hydrocarbons, which are the cause of leakage currents in $\mathrm{SiO}_{2}$. As alternative to oxygen cosupply, annealing steps at $600-800{ }^{\circ} \mathrm{C}$ for $60 \mathrm{~s}$ in forming gas $\left(\mathrm{N}_{2} / \mathrm{H}_{2}\right.$ mixture) were applied, reducing the number of negative oxide charges in 2 nm thick FEB deposits to zero. ${ }^{476}$ The above values compare to near room temperature CVD fabricated $25 \mathrm{~nm}$ thick films from TEOS having a resistivity $\rho=1 \times 10^{13} \Omega \mathrm{cm}$ and a leakage current density of $1.8 \times 10^{-10} \mathrm{~A} / \mathrm{cm}^{2}$ (at $1 \mathrm{MV} / \mathrm{cm}$ ), and a breakdown field of $7.2 \mathrm{MV} / \mathrm{cm}^{477} \mathrm{~A}$ metal-insulator-metal diode, where the insulator was fabricated by FEB contamination with a resistivity of $\rho=1$ $\times 10^{11} \Omega \mathrm{cm}$, was shown in Ref. 478 .

FEB deposited resistors from hydrocarbon backpressure gas and precursors of $\mathrm{MeCpPt}(\mathrm{Me})_{3}, \mathrm{Me}_{2} \mathrm{Au}(\mathrm{tfa}) \mathrm{W}(\mathrm{CO})_{6}$, and $\mathrm{Me}_{2} \mathrm{Au}(\mathrm{acac})$ are reported in Refs. 259, 373, and 479482. The carbon to metal content in these deposits is used to tune the resistance in the ohm to megaohm range. Hoyle et $a .^{233}$ developed a two stage deposition model involving a high resistivity intermediate dissociation product and a final low resistivity product to explain deposit thickness and resistivity measurements for FEB with $\mathrm{W}(\mathrm{CO})_{6}$. For FEB deposition with the precursor $\mathrm{AuClPF}_{3}$, rapid repetitive line scans gave a better electrical conductance, which can be attributed to the desorption of ligand fragments. ${ }^{333}$ FEB deposits from $\mathrm{W}(\mathrm{CO})_{6}$ with low incident energy (down to $60 \mathrm{eV}$ ) were four times better conducting than with $20 \mathrm{keV}$. $^{64}$

Resistance versus temperature measurements indicate an electron-hopping conduction mechanism due to the deposit substructure consisting of metal nanocrystals embedded in a carbonaceous matrix. A superconducting behavior below $5.5 \mathrm{~K}$ of FIB deposited wires from $\mathrm{W}(\mathrm{CO})_{6}$ was shown. $^{346}$ The resistance versus temperature behavior of wires from $\mathrm{MeCpPt}(\mathrm{Me})_{3}$ precursor was shown to rely on a variable range hopping mechanism with Coulomb oscillations for FEB deposits, whereas the resistance of FIB deposited wires hardly depended on temperature. ${ }^{483}$ In one case measurements with wires obtained from the same precursor were shown to be metallic for FEB deposition, whereas it was semiconducting for the FIB deposition due to $\mathrm{Ga}$ contamination. $^{484}$

\section{Electrical contacts}

Requirements for electrical contacts are low resistance and a work function match in case of contacts to semiconductors, often nanowires, to avoid Schottky barriers.

Delocalized (halo) deposits around the FEB or FIB written contact line can lead to an unwanted contamination or decoration of the nanowire or nanotube surface and might influence electrical measurements. The reason for the halo deposits with sizes in the micrometer range around the incident beam is due to incident stray ions and redeposition for FIB and due to back and forward scattered electrons for FEB processing. A comparison of halo deposits generated by focused beams of $30 \mathrm{keV}$ Ga ions and $10 \mathrm{keV}$ electrons during $\mathrm{MeCpPt}(\mathrm{Me})_{3}$ deposition showed a $3 \mu \mathrm{m}$ sized $\mathrm{Ga}$ and $\mathrm{Pt}$ halo and a $10 \mu \mathrm{m}$ carbon halo around the deposits. ${ }^{485}$ The electrical leakage resistance of such halo deposits was analyzed. ${ }^{486}$ In the case of FEB fabricated Hall sensors from $\mathrm{Co}_{2}(\mathrm{CO})_{8}$, the halo deposits were shown to be insulating and not to increase the active sensor area. ${ }^{323}$ Halo deposits during FEB induced deposition contact lines from $\mathrm{AuClPF}_{3}$ could be drastically diminished using low electron beam energies. ${ }^{334}$ Basically, the backscattered electron range (see Appendix A) gives the halo size in case of low-aspect-ratio $\leqslant 1$ FEB deposits. Thus a reduction of the incident electron energy to $1 \mathrm{keV}$ would reduce the halo size around the deposit below $40 \mathrm{~nm}$.

Contact resistances lower than with conventional $\mathrm{Cr} / \mathrm{Au}$ lift-off technique were obtained by FEB wiring single wall carbon nanotubes with the inorganic precursor $\mathrm{AuClPF}_{3}$ (Ref. 349) [see Fig. 52(a)]. The resulting pure metallic Au deposit has still a resistivity of 10-20 times of bulk gold due to the percolative grain structure. ${ }^{334,371}$ Pure Au core deposits surrounded by a carbon crust were obtained by adding water or oxygen as reactive gas during $\mathrm{FEB}$ with $\mathrm{Me}_{2} \mathrm{Au}(\mathrm{acac})$ 

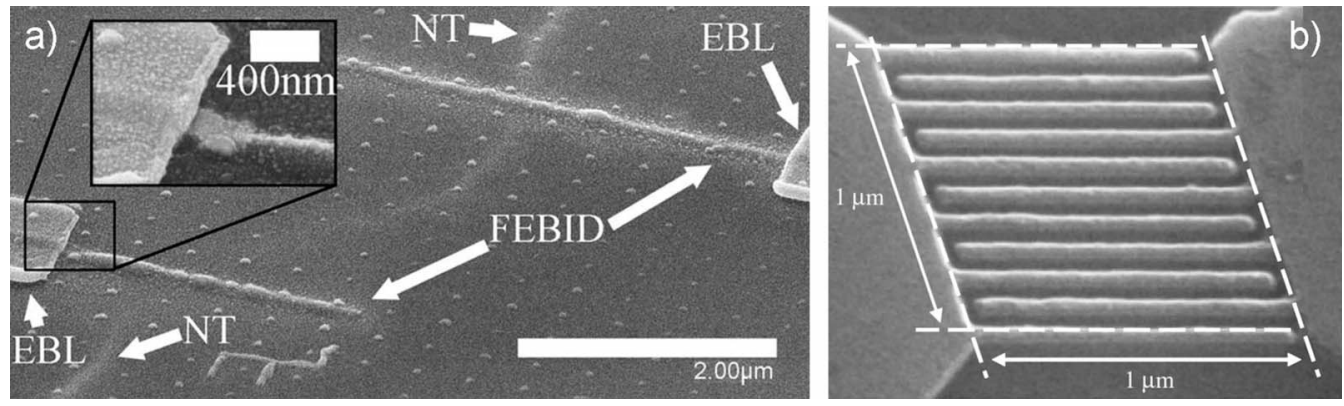

FIG. 52. FEB and FIB fabricated napoelectronic units. (a) FEB deposited gold lines (FEBID) to connect a single wall carbon nanotube (NT) to lift-off prefabricated gold electrodes (EBL) (from Ref. 349). (b) FIB $\left(\mathrm{Ga}^{+}\right) \mathrm{XeF}_{2}$-enhanced etching of Ti for interdigitated electrodes (from Ref. 232).

precursor. With the assumption that all current is carried in the core, resistivities of 2-30 times of bulk gold were estimated. $^{388,487}$ FEB deposited electrical contacts from $\mathrm{W}(\mathrm{CO})_{6}$ were also realized. ${ }^{344}$ FEB deposited contacts to gold nanowires from a $\mathrm{Pt}$ precursor (presumably $\mathrm{MeCpPt}(\mathrm{Me})_{3}$ showed worse specifications compared to standard e-beam lithography. ${ }^{488}$ High contact resistances in the order of $10 \mathrm{M} \Omega$ of FEB deposited contacts from $\mathrm{MeCpPt}(\mathrm{Me})_{3}$ to $\mathrm{SnO}_{2}$ nanowires were also reported. ${ }^{489}$

For $\mathrm{MeCpPt}(\mathrm{Me})_{3}$ a comparison gives $\rho_{\mathrm{FIB}}=20 \mu \Omega \mathrm{cm}$ and $\rho_{\mathrm{FEB}}=200 \mu \Omega \mathrm{cm}^{490}$ However, the contamination and the damage caused by incident $\mathrm{Ga}$ ions in nanowires or carbon nanotubes with FIB are detrimental for investigations of electrical properties. On the other hand, for $\mathrm{GaN}$ nanowires it was found that FIB deposited Pt contacts, consisting of nanocrystalline $\mathrm{Pt}$ embedded in an amorphous matrix of $\mathrm{C}$ and $\mathrm{Ga}$, have a metal-like conductivity and low intrinsic resistivities. ${ }^{491}$ A roughly $2-3 \mathrm{~nm}$ amorphous contact interface was detected and attributed to create interface states that could pin the Fermi level within the nanowire, which in turn results in low contact resistances and low Schottky-barrier heights. Another study on GaN nanowires showed that FIB deposited contacts were initially nonlinear and quite resistive, but after annealing the contact resistance became quite linear and four orders of magnitude lower than before. ${ }^{492}$

Thermal annealing generally increases the metal content in the deposit at the expense of oxidized (and thus removed) carbon, which improves the electrical conductivity of FEB deposits. This was discussed in Sec. V B 9. Annealing FIB deposited tungsten wires by injecting a high electrical current density of up to $6 \times 10^{7} \mathrm{~A} / \mathrm{cm}^{2}$ leads to segregation and evaporation of FIB implanted Ga material. ${ }^{493}$

The potential of FIB induced deposition for free space wiring with nanoscale control was demonstrated. ${ }^{494}$ The deposited diamondlike carbon material showed a relatively high resistivity of $100 \Omega \mathrm{cm}$ at room temperature.

We can conclude that low resistance electrical contacts by FEB deposition can be obtained using carbon-free precursors or adding additional reactive gases to organometallic precursors. For specific materials, for example, GaN, FIB induced damage is not detrimental during contact deposition.

\section{Laboratory prototype devices}

Various micro and nanoelectronic devices have been fabricated using FEB and FIB techniques. The following are some examples.

(1) Interdigitated titanium nanoelectrodes, which can be used as gas or biosensors, were fabricated with $\mathrm{XeF}_{2}$ assisted FIB milling having a $50 \mathrm{~nm}$ width and spacing and a resistance of $>1 \mathrm{G} \Omega$ (Ref. 232) [see Fig. 52(b)].

(2) A lateral tunnel junction device showing FowlerNordheim characteristics and a single electron transistor using $\mathrm{WF}_{6}$ and FEB is reported in Refs. 288, 289, and 495 .

(3) Submicron Hall sensors made from $\mathrm{Co}_{2}(\mathrm{CO})_{8}$ precursor with FEB having a sensitivity of $1 \mathrm{~V} / \mathrm{AT}$, a resistance of a few kilo-ohms, a maximum bias current of $1 \mathrm{~mA}$, and room temperature magnetic-field resolution of $10^{-6} \mathrm{~T} / \mathrm{Hz}^{1 / 2}$ were shown in Ref. 323. FIB milling of nano-Hall sensors from doped semiconductor material GaAs:Si and $\mathrm{Au}$ films down to 100 $\times 100 \mathrm{~nm}^{2}$ active areas were demonstrated in Refs. 496 and 497.

(4) Combined FIB milling and ion implantation were used for the fabrication of high temperature superconductor Josephson junctions. ${ }^{498}$

(5) GaN nanostructures fabricated by FIB deposition at $600{ }^{\circ} \mathrm{C}$ using gallane quinuclidine precursor and reactive nitrogen radical gas showed near-band-edge emission from $\mathrm{GaN}$ and other luminescence attributed to defects and/or impurities. An improved fabrication method showed strong near-band-edge emission at $3.37 \mathrm{eV}$ from $\mathrm{GaN}^{392}$

(6) The feasibility of quantum wire fabrication was shown by FEB etching with $\mathrm{Cl}_{2}$ into GaAs/AlGaAs (Ref. 499) and by FEB deposition with $\mathrm{GaMe}_{3}$ and $\mathrm{AsH}_{3}$. ${ }^{500}$

An interesting application of electron and ion beams is the irradiation of nanostructured carbon materials. (This area has been reviewed in a recent article. ${ }^{501}$ ) Electron beam irradiation of carbon nanotubes and graphene films can cause them to be reconfigured. In addition when the single walled carbon 


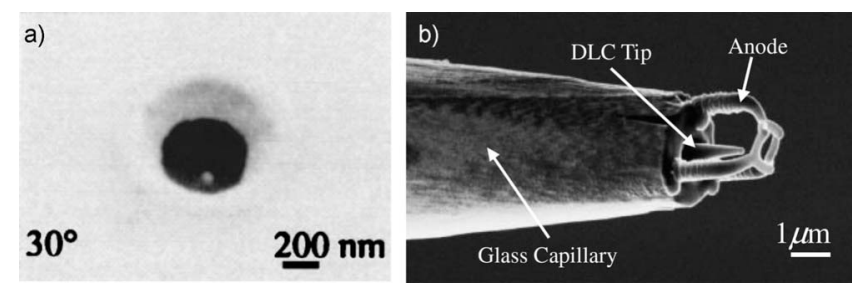

FIG. 53. FEB and FIB fabricated emitters. (a) FEB deposited emitter in a plate anode arrangement (Ref. 503). (b) FIB deposited emitter-anode arrangement on glass fiber (from Ref. 504).

nanotubes are FIB irradiated with doses between $10^{12}$ and $10^{15}$ ions $/ \mathrm{cm}^{2}$, the conductance of the carbon nanotube drops monotonically to zero. This was exploited by scanning two lines across a carbon nanotube in close proximity, thus forming a quantum dot showing Coulomb blockade behavior. ${ }^{502}$

\section{F. Field emitters}

The advantages of electron field emitters compared to thermal and Schottky emitters are high brightness, small energy spread, and good coherence. In applications such as flat panel displays, the emission characteristic of an individual emitter is not critical, whereas it becomes critical when (addressable) emitter arrays are used in future massive parallel electron beam lithography (or FEB deposition and etching) tool.

Due to its capability to deposit high resolution tips, FEB was predominantly applied in fabrication laboratory prototypes of field emitters. Specifications of field emitters obtained with different precursors can be found in numerous articles. ${ }^{364,365,505-520}$ It was found that FEB deposited emitters show high field enhancement, high emission currents at low voltage, and good beam confinement. Electron-hopping processes instead of Ohmic conduction allow for extraordinarily high current densities within these wires reaching more than $2 \mathrm{MA} / \mathrm{cm}^{2}$ (Ref. 343) to $20 \mathrm{MA} / \mathrm{cm}^{2} .{ }^{521}$

More recent work focused on emitter reproducibility in addressable field arrays, ${ }^{503,522}$ [see Fig. 53(a)], free electron lasers ${ }^{521,523}$ electron wave interference, ${ }^{524}$ and long term stability of emitters. ${ }^{525}$ A fully FEB deposited field emitter was presented in Ref. 508 and 526. The feasibility of producing cold field emitters using FIB induced deposition with differing precursors was demonstrated in Ref. 504 [see Fig. 53(b)].

\section{G. Mask fabrication for pattern transfer}

The requirements for FEB or FIB processed masks are the same as for lithography resists: stability against wet or dry etch. The advantage is that three dimensional profiles can be etched [see Fig. 54(a)].

Already in the 1990s FEB contamination deposits were used as resist for the fabrication of $8 \mathrm{~nm}$ tungsten line features ${ }^{7}$ and continue to be used as local anticorrosion masks for iron ${ }^{528}$ or as electrodeposition masks ${ }^{529,530}$ and wet etch masks for (110) Si (Ref. 531) or dry etch masks. ${ }^{272}$ FEB deposits from TTIP precursor were used as chlorine dry etch
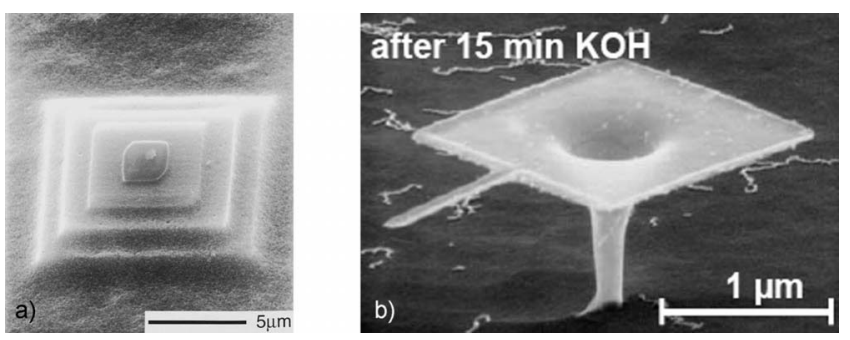

FIG. 54. Structures obtained after etching FEB and FIB masked substrates. (a) $\mathrm{Si}$ structure formed by $\mathrm{CF}_{4}$ plasma etching using a $\mathrm{FEB}$ deposited mask from $\mathrm{W}(\mathrm{CO})_{6}$ (Ref. 65). (b) Ga doped Si structure obtained by $\mathrm{Ga}^{+}-\mathrm{FIB}$ implantation and successive $\mathrm{KOH}$ wet etch (Ref. 527).

mask of GaAs. ${ }^{532}$ An etch rate of $0.16 \mathrm{~nm} / \mathrm{s}$ of FEB deposits from $\mathrm{W}(\mathrm{CO})_{6}$ in $\mathrm{CF} 4$ plasma was demonstrated, ${ }^{65}$ which is a 38 times slower etch rate than $\mathrm{Si}$ and gives a good selectivity. FEB etching with chlorine was used to locally remove the (natural) oxide layer on GaAs to allow for a subsequent etch process. ${ }^{53-535}$

A method of patterning $n$-type GaAs, InP, InGaAs, and InGaAsP by photoelectrochemical etching in conjunction with a $20 \mathrm{kV} \mathrm{Ga}^{+}$-FIB is reported. ${ }^{49}$ The FIB is used to produce a desired damage pattern on the surface, which is more etch resistant than the nondamaged material due to introduced electron-hole pair recombination centers. The etch stop appears at ion densities of $(3-5) \times 10^{10} \mathrm{ions} / \mathrm{cm}^{2}$, i.e., the sensitivity of this process was extremely high such that writing rates of nearly $1 \mathrm{~mm}^{2} / \mathrm{s}$ were obtained. FIB Ga implantation into boron doped $\mathrm{Si}$ at a concentration of 1 $\times 10^{15}$ ions $/ \mathrm{cm}^{2}$ or higher creates an etch stop to $\mathrm{KOH}$ wet etching. ${ }^{536}$ Such concentrations are also reached in the sidewalls of FIB milled structures, which makes fabrication of tailored three dimensional nanostructures possible ${ }^{527}$ [see Fig. 54(b)]. FIB deposition from phenanthrene precursor was used to fabricate three dimensional molds for nanoimprint lithography into hydrogen silsequioxane. ${ }^{537,538}$ FIB milled pits in $\mathrm{Al}$ were used to guide the anodization growth process of nanochannels. By this means a hexagonal close packed array of $12 \mathrm{~nm}$ holes in an alumina film with $100 \mathrm{~nm}$ spacing has been created. ${ }^{539}$

\section{H. Mechanical applications}

Gas-assisted FEB and FIB are often used as attachment tools for carbon nanotubes or nanowires onto AFM tips or microelectromechanical system structures ${ }^{540-546}$ [see Fig. 55(a)]. Cutting of carbon nanotubes with FEB and water vapor was reported in Ref. 547.

Often FIB induced deposition and milling is used for TEM lamella lift-off. Tweezers and grippers as shown in Figs. 55(b) and 55(c) were fabricated by FIB (Ref. 549) and FEB ${ }^{548}$ A FIB encapsulated pressure sensor is reported in Ref. 31. A small mechanical rotor unit was produced by FIB deposition $^{550}$ [see Fig. 55(d)] and an electromechanical switch consisting of a free space wired coil and a nanowire were reported. ${ }^{551}$ The small interaction volume of Ga ions at 

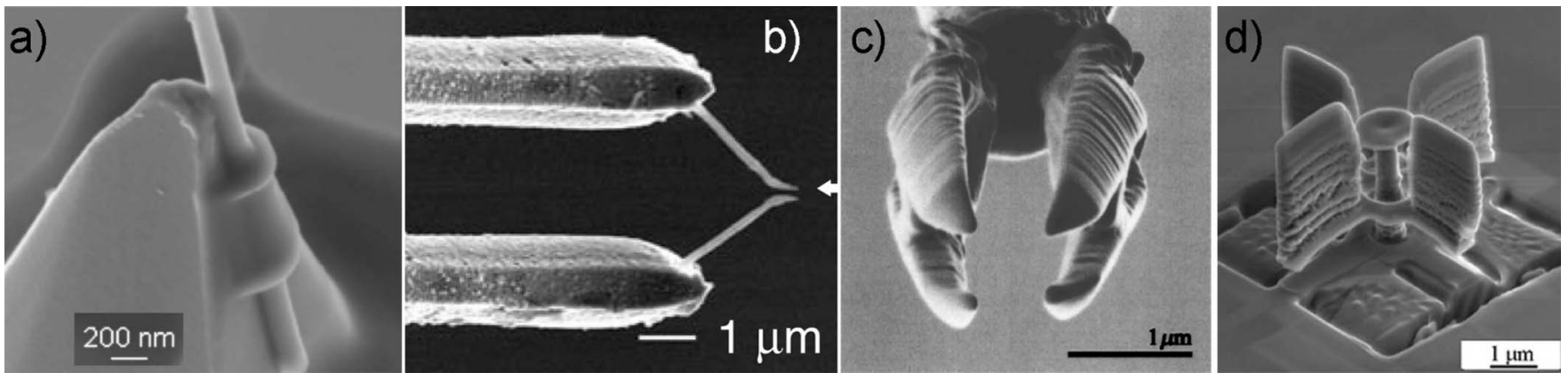

FIG. 55. FEB and FIB fabricated mechanical structures. (a) FEB deposited clamp to fix a nanowire to a AFM cantilever pyramid (Ref. 543). (b) Nanotweezers with $25 \mathrm{~nm}$ resolution (Ref. 548). (c) FIB deposited electrostatically actuated gripper (Ref. 549). (d) FIB deposited four wing rotor (Ref. 550).

high energies and the high deposition rates are quite favorable for the fabrication of these complex three dimensional structures.

Generally, FEB or FIB attachments of carbon nanotubes or nanowires can be made large enough to be stable against mechanical constraints applied to the attached structures. The mechanical quality of the fixation for vibration experiments was investigated in Ref. 552. Young's moduli of FEB and FIB deposits from phenanthrene were found to have an opposite dependency on incident beam energy. For $\mathrm{Ga}^{+}$-FIB deposits Young's modulus increased with increasing energy (from 20 to $90 \mathrm{GPa}$ for 5-30 keV), whereas for FEB deposits Young's modulus decreased (from 55 to $19 \mathrm{GPa}$ for $5-30 \mathrm{keV}) .{ }^{553}$ The density and Young's modulus of FIB deposits from phenanthrene depend on the deposition rate; the highest value was $600 \mathrm{GPa} .^{431,554}$ The mechanical properties of FIB deposits from $\mathrm{W}(\mathrm{CO})_{6}$ gave a Young's modulus of $300 \mathrm{GPa}$ and a density of $13 \mathrm{~g} / \mathrm{cm}^{3} .{ }^{432}$ FEB deposits from paraffin were investigated: the typical deposit was found to be hydrogenated amorphous carbon having more $s p^{2}$-than $s p^{3}$-bonded carbon. Nanoindentation tests revealed a hardness of about $4 \mathrm{GPa}$ and an elastic modulus of 30-60 GPa, depending on the accelerating voltage. ${ }^{543}$ Tensile strength measurements of FEB deposits from $\mathrm{W}(\mathrm{CO})_{6}$ and $\mathrm{Co}_{2}(\mathrm{CO})_{8}$ gave values in the order of $1 \mathrm{GPa}^{433} \mathrm{FEB}$ pillar deposits from (hfa)Cu-VTMS showed an increase in density with increasing dose (from 2 to around $4 \mathrm{~g} / \mathrm{cm}^{3}$ ), whereas FEB deposits from TMOS showed a constant density of $1.9 \pm 0.3 \mathrm{~g} / \mathrm{cm}^{3}$ with electron dose. Densities of deposits from hydrogen-free precursors, such as $\mathrm{Co}_{2}(\mathrm{CO})_{8}$ and $\mathrm{Rh}_{2} \mathrm{Cl}_{2}\left(\mathrm{PF}_{3}\right)_{4}$, are linearly related to their composition. ${ }^{91}$

\section{Biorelated applications}

Employment of gas-assisted FEB and FIB processes in biology are relatively new. Applications comprise nanopore membranes, which are being pursued as a potential method for high-speed DNA sequencing.

Nilsson ${ }^{555}$ reported a FIB process, which includes FIB milling of pores into silicon nitride membranes and their successive diameter reduction by gas-assisted FIB deposition using tetraethoxysilane and water [see Fig. 56(a)]. Danelon $^{557}$ used a subnanometer accuracy FEB deposition process for nanopore diameter reduction with real-time con- trol. Sub-5-nm diameter milling into $\mathrm{SiC}$ membranes with a highly focused $\mathrm{Ga}^{+}$beam ( $35 \mathrm{keV}, 2 \mathrm{pA}, 5 \mathrm{~nm}$ FWHM) was demonstrated [see Fig. 56(c) ]. ${ }^{55}$ A robust electronic detector consisting of a single nanopore in a $\mathrm{Si}_{3} \mathrm{~N}_{4}$ membrane, capable of registering single DNA molecules in aqueous solution, was fabricated: ${ }^{558}$ A $3 \mathrm{keV}$ Ar ion beam was used for controlled closing of nanopores down to $1.8 \mathrm{~nm}$ by creating diffusive adatoms.

Subelectron-beam diameter etching of a carbon model membrane using water or $\mathrm{XeF}_{2}$ was demonstrated using time-resolved stage current measurements. ${ }^{559}$ Other applications include neural interface systems. Hoshino ${ }^{556}$ presented the FIB deposition of a regenerative electrode made of diamondlike carbon [see Fig. 56(b)]. Although Ga contamination is an issue in FIB deposition, the deposits showed a sufficiently low cytotoxicity for growing nerves in vitro. A FIB deposited cell wall cutting tool for subcellular cutting operations without damaging underlying organelles as well as capture tool for organelles were demonstrated. ${ }^{560}$

\section{SUMMARY AND OUTLOOK}

\section{A. FEB versus FIB}

In certain respects FEB fabrication and FIB fabrication are complimentary. The situation for gas-assisted processing can be summarized as follows.

FIB has generally a higher yield in deposition and etching but generally damages the area addressed and contaminates it with gallium. Also selectivity is difficult to achieve. FEB on the other hand produces no (or substantially less) damage or

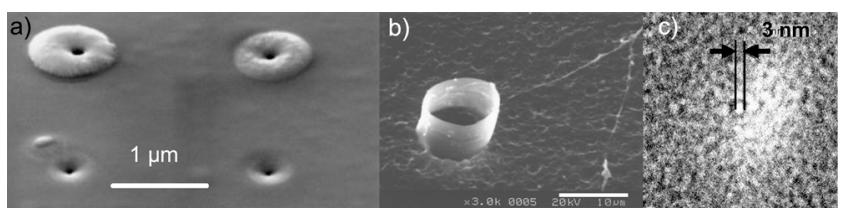

FIG. 56. FEB and FIB fabricated biological structures. (a) Nanopore diameter tuning with FIB deposition from tetraethoxysilane (TEOS). Nanopores were FIB milled into membrane (Ref. 555). (b) Biocompatible FIB deposited diamondlike carbon microtube with an in vitro grown nerve (Ref. 556). (c) Hole of $3 \mathrm{~nm}$ diameter milled by FIB into a $20 \mathrm{~nm}$ thick SiC membrane (from Ref. 55). 
contamination and can have high selectivity, but is slower, produces low metal content deposits, and cannot be used to remove inert materials such as gold.

How inherent are the disadvantages? For FEB it was shown that smart chemistry can improve the metal content close to $100 \%$ by using inorganic precursors and additional reactive gases. The same approach should allow finding etchants for most materials. In how far the yield can be improved with this concept remains an open question, but clearly the exposure dwell time of the electron or ion beams has to be reduced to a minimum (see Table IX). Especially, for small structures the throughput is often limited by gas replenishment and not by the yield [Fig. 30(b)]. It can be concluded that for FEB the disadvantages are not inherent but rather represent the present state of the art of precursor research. For $\mathrm{Ga}^{+}-\mathrm{FIB}$ the disadvantages are coupled to the heavy mass of ions and are thus inherent. However, replacing the predominantly used Ga ions by lighter ions such as protons or helium or other noble gases might diminish the extent of damage and contamination, where they prove detrimental in nanofabrication.

\section{B. Process regimes}

The understanding of the concept of process regimes in gas-assisted FEB and FIB is of utmost importance. As discussed in Secs. III and IV they govern the resolution, composition, and throughput of the process. The understanding and control of process regimes are prerequisites for the further technological development of FEB and FIB technologies as well as the correct scientific interpretation of experimental results. Correct interaction parameters of charged particles with molecules and the (maximum) process yield will be only obtained when working in the charged particle limited regime, the conditions of which were specified in Sec. III E. Furthermore, the beam current and dwell time should be reduced to avoid unwanted side reactions (like organic fragment fixation before desorption) to take place.

The molecule limited regime becomes important in FEB processes involving several species of adsorbates, either deliberately introduced as gases, formed as intermediates, or present as background contamination (hydrocarbons and water). What matters is to find the irradiation conditions for which one of the molecule species becomes limited with respect to the others. Then deposit properties can be changed as well as switching between deposition and etching be observed. For FIB processing switching between deposition and etching can also occur for one single molecule species due to the physical sputter action of the incident ions representing the second "species."

Regime transitions from molecule limited to electron/ion limited with increasing distance from the beam center occur within the incident beam profile and its spatially related emitted energy spectrum (although passing often unnoticed). The geometry of the resulting structures (rings or holes for stationary beam) is self-limiting, meaning that no precise exposure control is needed to reproducibly fabricate such structures. On the other hand it also means that deposits can be composed of a core and mantle (crust) both having different physical properties due to different composition. Finding exposure conditions (beam parameters, vacuum conditions, or molecules) that avoid this substructure is very important for depositing pure material.

\section{Precursor molecules and deposit purity}

A large variety of molecules is used in gas-assisted FEB and FIB processing as was shown in Sec. V. For the majority of FEB and FIB experiments, the energies of the incident beam and the energy spectrum of the emitted electrons or surface excited atoms will not lead to breaking of one specific bond (for instance, desirably the metal-ligand bond) in the adsorbed molecule but will quite unselectively break other bonds, too. However, the very rapid energy redistribution in excited molecules often favors dissociation of the weakest bonds (if other electronic relaxation can be avoided). This redistribution also limits the process window for selective bond scission of higher energy bonds in the molecule. In fact, the dissociation of any bond is accomplished by the electronic excitation of the whole molecule. The resonant dissociative electron attachment discussed in Sec. II B was shown to be selective with respect to the molecule energy states of alcohols at incident energies below $10 \mathrm{eV}$. If this state selectivity could be proven for metal organic molecules, one would be able to tune the molecule fragmentation and thus the deposit composition.

Presently, sub-1-keV scanning beam systems can be only achieved at very low lateral resolution. In principle, scanning tunneling microscopes could provide such low-energy electron beams at $10-50 \mathrm{pA}$ tunneling current. In any case, the dissociative electron attachment mechanism, which is employed at such low energies for molecule dissociation, would still need further investigation for its specific dissociation action on the desired ligand bond to produce pure material.

Presently, there are three conceptual approaches followed for obtaining pure deposit material with $\mathrm{keV}$ beams. They comprise the use of (1) inorganic molecules, (2) additional reactive gases, and (3) UHV systems.

(1) Inorganic molecules are presently limited in variety to a few metal hydrides, metal halides, and metal trifluorophosphines (see Secs. V B 2, V B 3, and V B 5), respectively. The stability and the handling of these precursors are delicate and it would be desirable for the future to have less sensitive (moisture, light, and spontaneous decomposition) and less toxic molecules at hand. As simple rule of thumb, the precursor with highest probability of obtaining the desired product is a poorly stable compound, which decomposes thermally at a temperature slightly above the substrate temperature to the wanted product. This precursor should not contain polymerizable ligand atoms such as $\mathrm{C}, \mathrm{S}$, and, to a lower extent, $\mathrm{P}$.

(2) Adding reactive gases forms volatile reaction products with unwanted codeposits, mostly hydrocarbons. Using an oxidizing reactive gas additionally removes oxygen deficiencies of the deposited material and 
worked very well for oxide deposition. A reducing reactive gas should be used if metal oxides must be avoided. Examples were given in Sec. V C.

The use of UHV systems for pure metal deposition proved very recently successful for iron [see Sec. V B 4 (a)]. It will be the issue of further research if this is the case also for other molecules.

There are also methods for reducing the carbon content: a simple method for reducing hydrocarbon contamination in gold and copper during the deposition process was shown to be the deposition on a heated stage to help volatilize the reaction products (see Sec. VI B). When heating occurred by the charged particle beam, pure metal deposits could be also obtained. However, the lateral process resolution is then given by the thermally activated volume being in general much larger than the finely focused beam (see Sec. II F). Another method to obtain pure material is ex situ treatment at elevated temperatures with or without reactive gases, which was discussed in Sec. V B 9.

Finally, we would also like to note that functional deposit material can be obtained using carbon containing metal precursors. The resulting nanocomposite material, mostly metal nanocrystals embedded in a carbonaceous matrix, has properties within one to three orders of magnitude close to that of pure metal and the stabilizing function of the carbon matrix against mechanical load or chemical attack. Many of the laboratory prototype applications presented in Sec. VII are operative with this type of nanocomposite material.

\section{Fragmentation channels and reaction paths}

Although a variety of impressive applications has been brought about by gas-assisted FEB and FIB processes as discussed in Sec. VII, there is, from a scientific point of view, an important lack in understanding of the beam induced chemistry. On the one hand there is a deep fundamental insight into electron stimulated chemistry in adsorbed molecules (and even more in the gas phase). This knowledge is however very specific with respect to very few molecules and experimental conditions, which are highly idealized (ultrahigh vacuum, crystallographic and clean surfaces, and low-energy electron beams below $100 \mathrm{eV}$ ). On the other hand there are gas-assisted FEB and FIB processes, which mostly proceed above $1 \mathrm{keV}$ incident energy (involving a secondary low-energy spectrum due to interaction), perform on surfaces being continuously deposited or etched, and operate with local pressures far from ultrahigh vacuum. There is some similarity to chemical vapor deposition (CVD); however, there is a major difference: classical low pressure or atmospheric pressure chemical vapor deposition operates at temperatures above the thermal decomposition temperature of the precursor molecule in order to guarantee rapid desorption of ligands or other undesired fragments. The temperature range of FEBIP and FIBIP is often such that dissociation fragments are irreversibly adsorbed and immobile. Furthermore, the pressure range is low so that there is no equilibrium between gas and solid phase. Hence, the question is how can the concepts of surface science and CVD help to understand the gas-assisted FEB and FIB process? This is a challenge for scientists from all fields. One step forward into this direction has been done by using ultrahigh vacuum FEB setups, which allow operating under well defined atmospheres (mainly no residual $\mathrm{H}_{2} \mathrm{O}$ partial pressure, see Secs. II C 2, II D 1, and V A 1). Another step has been done in using low-energy beams (although less focused), which are in the energy range of the secondary electron emitted spectrum.

We feel that work in this direction is about to start in a systematic way, which will lead to a deeper understanding of the mechanism the gas-assisted FEB and FIB processes rely on. Probably, this will be in detail very much molecule dependent but will result in future in a general understanding improving the purity of the deposits, the resolution, and also the throughput of the process.

\section{E. Resolution}

The best lateral resolutions are reported for aspect ratio structures below or near 1. sub-5-nm resolution of lowaspect-ratio deposits by FEB on bulk $\mathrm{Si}$ was demonstrated in a (high resolution) SEM. ${ }^{243}$ The current world record is a $0.72 \mathrm{~nm}$ small deposit (full width at half maximum) obtained on a membrane in a scanning transmission electron microscope. ${ }^{92}$ Deposition at this scale was found to be determined by a random process: deposits do not exactly nucleate at the irradiated spot. The possibility to deposit one single molecule using the molecule limited regime and an in situ feedback control signal was suggested. ${ }^{561}$ MC simulations also suggest that $1 \mathrm{~nm}$ deposits could be obtained on bulk substrates. $^{237}$

For high-aspect-ratio $(>1)$ deposition, the generation of secondary electrons (along the scatter trajectories of incident electrons) exiting the deposit volume leads to pillar diameters in the order of 5-20 nm (for coaxial exposure) according to simulations performed in the electron-limited regime. Experimentally, best pillar diameters obtained are around $50-80 \mathrm{~nm}$ and best apex diameters were around $10 \mathrm{~nm}$. Depositing freestanding rods by moving the electron beam laterally in one direction from the sample's edge into empty space produced at best $5 \mathrm{~nm}$ rod and apex diameters. Presently, the best etch resolutions were in the order of 10-40 nm for metal membranes as discussed in Sec. VII A. Generally, gas-assisted FIB has a lower resolution than FEB. Apart from the larger FIB diameter, we attribute this to the molecule-limited regime in the central beam irradiated region.

\section{F. Models}

Models for process rates as well as for deposit and etch shapes can be classified into continuum models and Monte Carlo simulations (or a mixture of both). The beauty of Monte Carlo models is that incident and secondary electrons or incident ions and cascade ions can be traced anywhere in space with their remaining energy. Some assumptions and 
limits with regard to secondary electron generation were discussed in Sec. II A. Things become really complicated when the interaction with adsorbed molecules must be described quantitatively. First, it is difficult to decide which dissociation channel (see Sec. II B) is responsible for deposition and, second, in most cases data on the related cross section are missing. A pragmatic approach is followed, which takes available data from the gas phase. There is also a lack of data for the surface diffusion coefficients and residence times. As a consequence, the simulations should not be overinterpreted in terms of generality or quantity. However, relative trends in three dimensional shape evolutions can be qualitatively predicted. Continuum models have the beauty of simplicity (without being oversimplifying). Their applicability limit is near the $1 \mathrm{~nm}$ scale where matter starts to behave discontinuously. Simple, low-aspect geometries can be treated reliably. They allow for easy implementation of exposure parameters (dwell time, refresh time, electron/ion flux, beam profile, etc.) and are very useful in determining unknown parameters such as the deposition (or etch) cross section and the process yield (both at a given incident energy), molecule adsorption, desorption, and diffusion. General scaling laws could be predicted as well as the classification into the three process regimes performed (see Sec. III).

For the future, we would like to suggest that any simulation or calculation should be carefully checked against real experiments and that underlying assumptions together with their consequences should be stated. This is not always easy since gas-assisted FEB and FIB deposition and etching comprises many physicochemical processes.

\section{G. Fundamental issues}

Although many FIB and FEB fabrication techniques have been developed and the fundamental aspects of the processes have been studied, a number of scientific as well as technological challenges still remain.

(1) For many of the precursor molecules, the irradiation chemistry is unknown. This includes dissociation channels, polymerization channels, and intermediate production. Since FEB and FIB interaction with matter produces an energy spectrum, these reactions can also occur simultaneously.

(2) For many precursor gases the role of surface kinetics (adsorption, desorption, and diffusion) is not understood nor characterized. Dissociative adsorption with ligand transfer to the substrate might play a dominant role in deposition reactions.

(3) The role of high-energy beams or focused low-energy beams has been explored only to a limited degree.

(4) The role of possible gas phase reactions and charging effects has been explored only to a limited degree.

(5) The role of substrate temperature has also only been exploited in a couple of instances.

The fundamental understanding and the potential of gasassisted FEB and FIB induced deposition and etching would be greatly improved starting investigations of these issues.

\section{H. Future prospects}

There are two limitations to the practical applications of ion beam and electron beam direct processing, which are implicit in what we have discussed so far: (a) because of the particle substrate interaction, the dimensions of high-aspectratio structures one can fabricate are larger than the beam diameter, and (b) because the fabrication by the beams is serial, the time needed to fabricate any structures is long and mass-production-type applications are impractical. There are two recent developments which may to some degree overcome these limitations.

\section{Helium ion beam}

Although the gas field ion source mentioned in Table II has been studied for many years and impressive performance has been demonstrated, its operation at cryogenic temperatures has always been difficult, and no practical helium ion column had been developed. A few years ago Alis Corp. (now part of Carl Zeiss SMT) in Peabody, MA announced a helium ion beam system with imaging capabilities superior to that of a scanning electron microscope. In this ion source the ions are emitted from a single tungsten atom of an oriented single crystal tip, thus enabling a subnanometer beam diameter. $^{40,562,563}$

The ion/substrate interaction of a helium ion can be thought of as intermediate between that of the electron and the heavier $\mathrm{Ga}^{+}$ion. It also loses energy mainly to the electrons in the substrate and does not produce collision cascades of the kind produced by gallium ions except near the end of its travel when it has lost most of its energy. Because of the vast mass difference between a helium ion and an electron, one may speculate that the area from which secondary electrons are emitted in the case of the helium ions is smaller than the area from which secondary electrons are emitted when an electron beam is incident. Moreover, the secondary electron yield of the ions is between 3 and 10 for most materials and is higher than that of electrons. ${ }^{40}$ The nanofabrication potential of He ions has, to our knowledge, not been explored. However, beam induced deposition has been observed with a broad $5 \mathrm{keV}$ He ion beam. ${ }^{57}$ The net deposition yield (dissociation yield minus sputter yield) from dimethylgold hexafuoroacetylacetonate was 0.24 , while the sputter yield was 0.15 . For heavier ions, for example, krypton (close in mass to $\mathrm{Ga}$ ), these numbers are 2.8 and 9.3 at $5 \mathrm{keV}$. Even just sputtering data for $\mathrm{He}$ ions are rare. Sputter yield of $\mathrm{Ag}$ at $10 \mathrm{keV}$ is 0.4 for $\mathrm{He}$ ions compared to 14.8 for $\mathrm{Kr}$ ions. ${ }^{68}$ Clearly sputtering and adsorbate dissociation do occur and there may be reason to expect that the surface/ion interaction may be more localized and therefore the fabricated structures may be closer to the beam diameter.

\section{Projection maskless patterning}

Ion projection lithography was actively considered and developed as one of the "next generation lithographies" to replace optical lithography for chip manufacturing. In this technology a broad beam of ions back illuminates a stencil 
mask, and the image of the mask is demagnified by an ion optical column onto the substrate to expose resist. ${ }^{564}$ For a review of this topic, see Ref. 565.

A potentially useful outgrowth from the ion projection lithography has recently been demonstrated. ${ }^{566}$ The stencil mask is replaced by a programable aperture mask, which produces a large number of beamlets each of which can be turned on or off. The image of this aperture mask is demagnified by $200 \times$ onto the sample. Thus $3.5 \mu \mathrm{m}$ mask openings produce $17.5 \mathrm{~nm}$ spots on the sample. For $10 \mathrm{keV}$ argon ions the total maximum current is quoted as $90 \mathrm{nA}$ and is limited by ion-ion repulsion. The beamlets are turned on and off in a programed fashion so as to produce the desired pattern as the wafer moves under the beam-sort of like an ion beam dot matrix printer. To produce a comparable dimension spot with a FIB system, the current in a single beam would have to be two to three orders of magnitude smaller. Thus the throughput in fabricating a given pattern with the multibeam system would be 100 to 1000 times higher. At higher beam energies and with lighter ions, a higher total current can be passed down the column before space charge effects come into play. For example, an earlier analysis of multibeam writing suggests that the total current of $3 \mu \mathrm{A}$ can be used. For electrons, of course, the current can be even higher. ${ }^{567}$

Projection maskless patterning, if developed, may well make many of the electron and ion beam fabrication processes we have discussed usable in commercial production of nanodevices.

\section{ACKNOWLEDGMENTS}

The authors would like to thank V. Friedli and T. Bret for a critical reading of the article and K. Edinger, J. Mulders, V. Callegari and S. Babin for material and data they provided. I.U. acknowledges financial support from the EU project NanoHand. To direct inquiries from readers and for proper attribution: the fundamentals and models of the processes, the organization of all the data, and descriptions of many of the applications are due to I.U., the gas chemistries are due to P.H., and J.M. contributed to the overall content and conception of the article, particularly the FIB material. In spite of search engines and the large number of references, the authors may still have missed important work. The authors apologize if this is the case and ask to be informed.

\section{APPENDIX A: RANGES OF ELECTRONS AND IONS}

Typical ranges of interaction and distributions will be presented via analytical expressions and/or Monte Carlo simulations. Although the analytical expressions are often referred to as gray numbers, ${ }^{53}$ they allow for a more physical understanding of the scaling of the excitation volume with respect to incident energy and target composition (elements).

Due to the energy loss electrons and ions are decelerated.

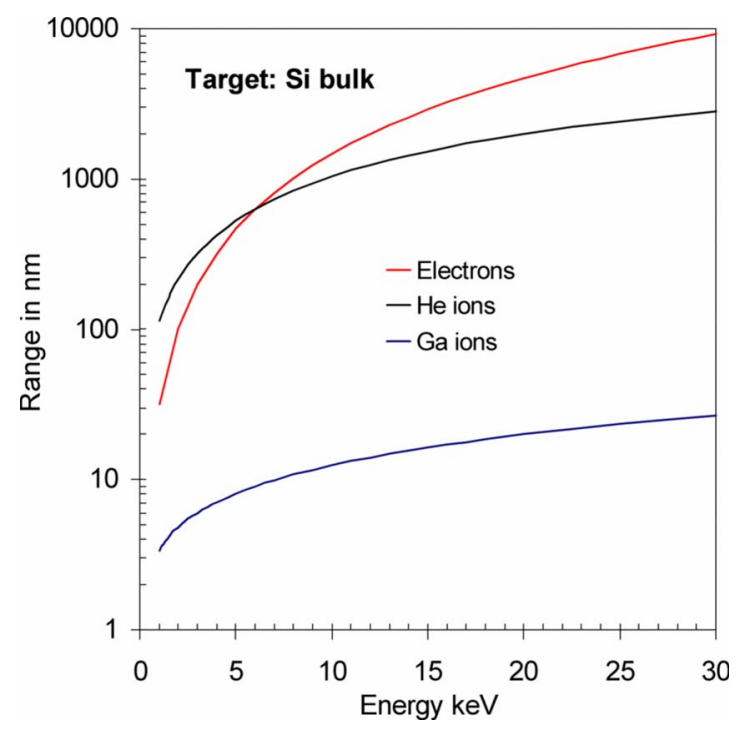

FIG. A1. Electron range $R_{E}$ [Eq. (A1)] and projected ranges of ions in bulk silicon according to SRIM (Ref. 73).

Electrons come to rest within a volume, the dimension of which can be estimated by the parametrized KanayaOkayama electron range $R_{E}$, (see also Fig. A1), ${ }^{568}$

$$
R_{E}=2.76 \times 10^{-4} \frac{M(\mathrm{~g} / \mathrm{mol}) E^{5 / 3}(\mathrm{eV})}{Z^{8 / 9} \rho\left(\mathrm{g} / \mathrm{cm}^{3}\right)} \quad(\mathrm{nm}) .
$$

This equation shows a good fit with experimental data down to $5 \mathrm{keV}$. For lower energies a dependence $R_{E}(E) \sim E^{1.3}$ was found. ${ }^{569}$ For bulk silicon the electron range is presented in Fig. A1 and extends from approximately $30 \mathrm{~nm}$ at $1 \mathrm{keV}$ to $10 \mu \mathrm{m}$ for $30 \mathrm{keV}$. Ranges of ions depend heavily on the ion/target elements. Generally, Ga ions have up to two to three orders of magnitude smaller range than electrons or low mass ions such as $\mathrm{He}$.

For the maximum depth of x-ray generation, Eq. (A1) can be adapted by introducing a reduced energy term $\left(E-E_{x}\right)^{5 / 3}$, where $E_{x}$ represents the specific energy to generate an x-ray photon in a given material. This energy ranges approximately between 0.5 and $10 \mathrm{keV}$ for $K, L$, and $M$ shell transitions.

An analytical expression for lateral electron straggling is known as electron beam broadening due to forward scattering and given in parametrized form as ${ }^{45}$

$$
x_{\mathrm{rms}}(\mathrm{nm})=3.32\left(\frac{\rho\left(\mathrm{g} / \mathrm{cm}^{3}\right)}{M(\mathrm{~g} / \mathrm{mol})}\right)^{1 / 2} \frac{Z}{E(\mathrm{eV})} t(\mathrm{~nm})^{3 / 2},
$$

with $t$ as the film thickness. This analytical expression holds within the first 25 collisions.

The dimension of the exit surface of backscattered electrons can be characterized by the radius $R_{\mathrm{BSE}},{ }^{568}$

$$
R_{\mathrm{BSE}}=1.1 R_{E} \gamma /(1+\gamma),
$$


with $\gamma=0.19 Z^{2 / 3}$ and $R_{E}$ as defined in Eq. (A1).

A unique formula for the range of a given ion species impinging on a target can not be found in literature. ${ }^{68}$ Instead Monte Carlo range algorithms ${ }^{80,570}$ are frequently applied to calculate ranges (see Fig. A1).

\section{APPENDIX B: ELECTRON AND ION ENERGY LOSSES AND STOPPING POWERS}

For electrons the total energy loss per trajectory path unit $d E / d s$ can be calculated from the modified Bethe-loss formula for energies $0.05 \mathrm{keV}<E<100 \mathrm{keV},{ }^{87}$

$$
\frac{d E}{d s}=\left\{\begin{array}{c}
\frac{2 \pi e_{0}^{4}}{\left(4 \pi \varepsilon_{0}\right)^{2}} \frac{N_{A} \rho Z}{M} \frac{1}{E} \ln \left(\frac{e^{1 / 2}}{2^{1 / 2}} \frac{(E+0.85 J)}{J}\right) \\
7.85 \times 10^{3} \frac{\rho\left(\mathrm{g} / \mathrm{cm}^{3}\right) Z}{M(\mathrm{~g} / \mathrm{mol}) E(\mathrm{eV})} \ln \left(1.166 \frac{(E(\mathrm{eV})+0.85 J(\mathrm{eV}))}{J(\mathrm{eV})}\right) \quad(\mathrm{eV} / \mathrm{nm}),
\end{array}\right.
$$

where $Z$ is the atomic number, $M$ is the molar mass, $\rho$ is the density, $N_{A}$ is Avogadro's number, $J$ is the mean ionization potential $\quad J=\left(9.76 Z+58.5 Z^{-0.19}\right) \mathrm{eV}, \quad \varepsilon_{0}=8.85$ $\times 10^{-12} \mathrm{~A} \mathrm{~s} / \mathrm{V} \mathrm{m}$, and $e_{0}=1.6 \times 10^{-19} \mathrm{~A} \mathrm{~s}$. The total stopping power $S(E)$ is obtained from the energy loss via the atomic density of the target $N=N_{A} \rho / M, d E / d s=N \cdot S(E)$.

For a compound $A_{m} B_{n}$ an average atomic number $Z_{\text {av }}$ $=\left(m Z_{A}+n Z_{B}\right)^{1 /(n+m)}$ is proposed to calculate its stopping power. $^{87}$

The analytical expressions for the electronic and nuclear stopping powers of ions are given in terms of the reduced energy $\varepsilon(E$ in $\mathrm{eV})$,

$$
\varepsilon=\frac{0.03255}{Z_{1} Z_{2}\left(Z_{1}^{2 / 3}+Z_{2}^{2 / 3}\right)^{1 / 2}} \frac{M_{2}}{M_{1}+M_{2}} E,
$$

where $Z_{1}$ and $M_{1}$ are the atomic number and mass of the ion and $Z_{2}$ and $M_{2}$ similarly for the target atoms. The nuclear stopping power is given by ${ }^{133,160,161}$ and references therein,

$$
S_{n}(E)=\frac{84.78 Z_{1} Z_{2}}{\left(Z_{1}^{2 / 3}+Z_{2}^{2 / 3}\right)^{1 / 2}} \frac{M_{1}}{M_{1}+M_{2}} s_{n}(\varepsilon) \quad\left(\mathrm{eV} \AA^{2}\right),
$$

where

$$
s_{n}(\varepsilon)=\frac{3.441 \varepsilon^{1 / 2} \ln (\varepsilon+2.718)}{1+6.355 \varepsilon^{1 / 2}+\varepsilon\left(6.882 \varepsilon^{1 / 2}-1.708\right)} .
$$

The electronic stopping power is given by

$$
S_{e}(E)=k \varepsilon^{1 / 2} .
$$

Which energy loss mechanism dominates depends on the mass of the ion. In Fig. B1 the energy loss of electrons, Ga ions, and $\mathrm{He}$ ions in silicon are presented graphically. Ions are stopped orders of magnitude more efficiently than electrons with the same energy. Basically, for electrons the total stopping power $S(E) \sim \ln (E) / E$, and for ions the electronic stopping power $S_{e}(E) \sim E^{1 / 2}$ and the nuclear stopping power $S_{n}(E) \sim E^{1 / 2} \ln (E) /\left(E^{3 / 2}+E^{1 / 2}-E\right)$.

\section{APPENDIX C: SKIRT DISTRIBUTION}

The integrated radial skirt distribution due to gas phase scattering is given as ${ }^{45}$

$$
R(r)=\int_{0}^{r} 2 \pi f_{s}(r) r d r / \int_{0}^{\infty} 2 \pi f_{s}(r) r d r,
$$

where $f_{s}(r)$ is the (MC-simulated) distribution of scattered electrons (or ions) arriving on the planar substrate after having passed the gas phase. Figure $\mathrm{C} 1$ shows such an integrated radial skirt distribution for a $20 \mathrm{keV}$ electron beam (with zero diameter), which scattered when passing through a $1 \mathrm{~mm}$ thick gas phase of $\mathrm{Co}_{2}(\mathrm{CO})_{8}$ molecules at its vapor pressure of 0.4 mbar. The scattered fraction amounts to $21 \%$ of the incident primary electrons. In comparison a Gaussian distribution having the same full width containing $50 \%$ of the scattered electrons is shown.

\section{APPENDIX D: DEPOSITED ENERGY}

There are different presentations of the energy deposited by focused incident electron or ion beams. Figure D1 shows

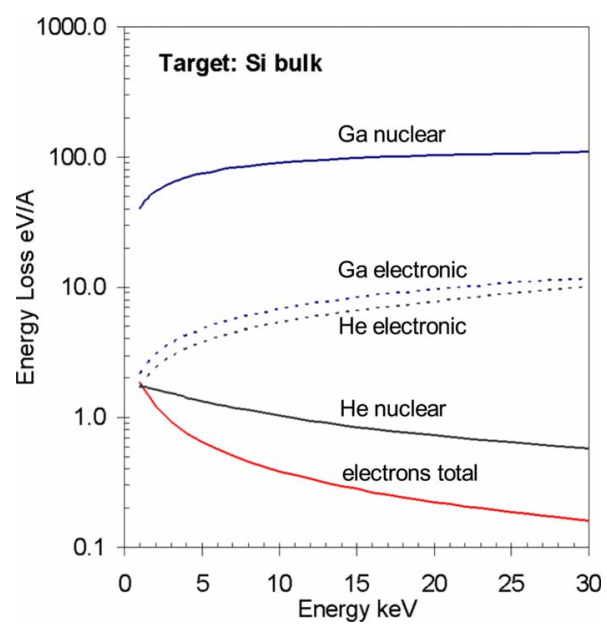

FIG. B1. Energy loss for electrons according to Eq. (B1) and nuclear/ electronic losses of $\mathrm{Ga}$ and $\mathrm{He}$ ions according to SRIM (Ref. 570). 


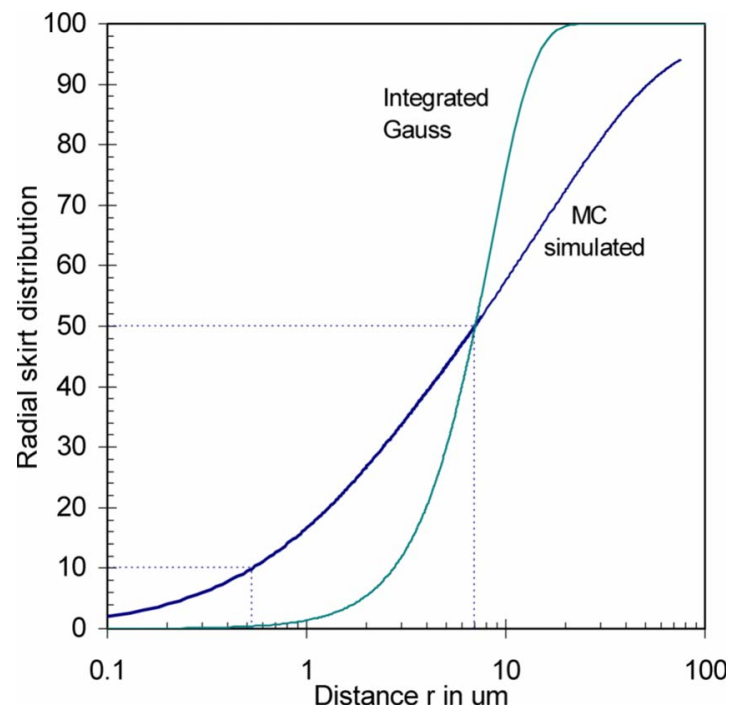

FIG. C1. Integrated radial distribution of scattered electrons incident on the substrate from a $20 \mathrm{keV}$ zero diameter beam passìng through $1 \mathrm{~mm}$ $\mathrm{Co}_{2}(\mathrm{CO})_{8}$ at $P=0.4$ mbar obtained from MC simulations (Ref. 81). The curve approaches $100 \%$ at larger distances. The scattered fraction is $21 \%$ of the PE. In comparison a Gaussian with the same FW50 is shown.

the radial energy distribution in a planar bulk $\mathrm{Si}$ substrate caused by an incident $20 \mathrm{keV}$ electron beam with zero diameter, which can be used as input for the heat term $H(r, z)$ in Eq. (2.15).

The energy distribution with depth $H(z)$ is obtained by integrating $H(r, z)$ over $r$, thus representing all the deposited energies in a plane slice at depth $z$ (changing the unit to eV/nm). Figure D2 shows a comparison between electrons and the ions $\mathrm{Ga}$ and He. For $H(z)$ parametrized expressions for the depth position of the lost energy maximum were established as a function of incident electron energy and material, ${ }^{568}$

$$
z_{E}=\frac{R_{E}}{2}\left(1-\left(\frac{1.1 \gamma}{1+\gamma}\right)^{2}\right)
$$

with $\gamma=0.19 Z^{2 / 3}$ and $R_{E}$ defined as in Eq. (A1). Equation (D1) gives a $20 \%$ agreement with MC simulations at low

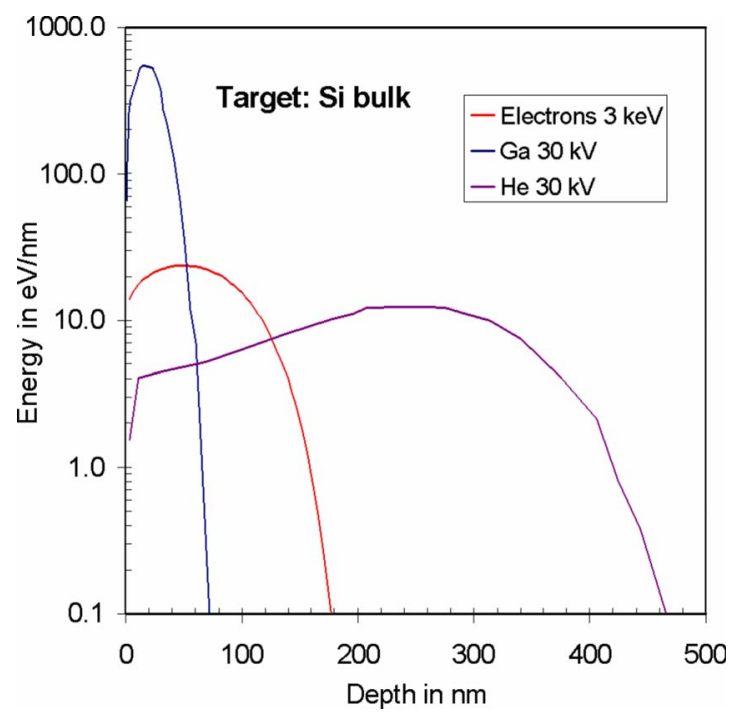

FIG. D2. MC simulation of dissipated energy $\mathrm{H}(\mathrm{z})$ for $3 \mathrm{keV}$ electrons (Ref. 81) and $30 \mathrm{keV} \mathrm{Ga}$ and $\mathrm{He}$ ions [SRIM (Ref. 73)]. Note the differences in energy and depth for the two ion species.

energy and 5\% agreement at $25 \mathrm{keV}$. An analytic expression for the deposited electron energy as a function of target depth is given in Ref. 569. As was the case for ion ranges, Monte Carlo simulations are frequently applied to calculate the distribution of deposited ion energy in the target (see Fig. D2).

\section{APPENDIX E: THE DIFFUSION PARAMETER $\boldsymbol{c}_{\mathbf{2}}$}

The diffusion contribution $C_{2}$ in Eq. (3.5) is given ${ }^{219}$

$$
C_{2}=K_{1}\left(\widetilde{\rho}_{\text {out }}^{-1}\right) \frac{1-\widetilde{\tau}^{-1}}{I_{0}\left(\widetilde{\rho}_{\text {in }}^{-1}\right) K_{1}\left(\widetilde{\rho}_{\text {out }}^{-1}\right)+\widetilde{\tau} I_{1}\left(\widetilde{\rho}_{\text {in }}^{-1}\right) K_{0}\left(\widetilde{\rho}_{\text {out }}^{-1}\right)},
$$

where $K_{n}$ and $I_{n}$ are modified Bessel functions and $\tilde{\rho}$ $=2 \rho / \mathrm{FWHM}_{B}$ represents the diffusion lengths normalized to the FWHM of $f(r)$. The surface diffusion length of molecules outside the irradiated area is defined as $\rho_{\text {out }}=\left(D \tau_{\text {out }}\right)^{1 / 2}$ and at the beam center $\rho_{\text {in }}=\left(D \tau_{\text {in }}\right)^{1 / 2}$. The dimensionless depletion is defined as $\tilde{\tau}=\tau_{\text {out }} / \tau_{\text {in }}$, i.e., as the ratio of the effective residence times outside the irradiated area and in the irradia-

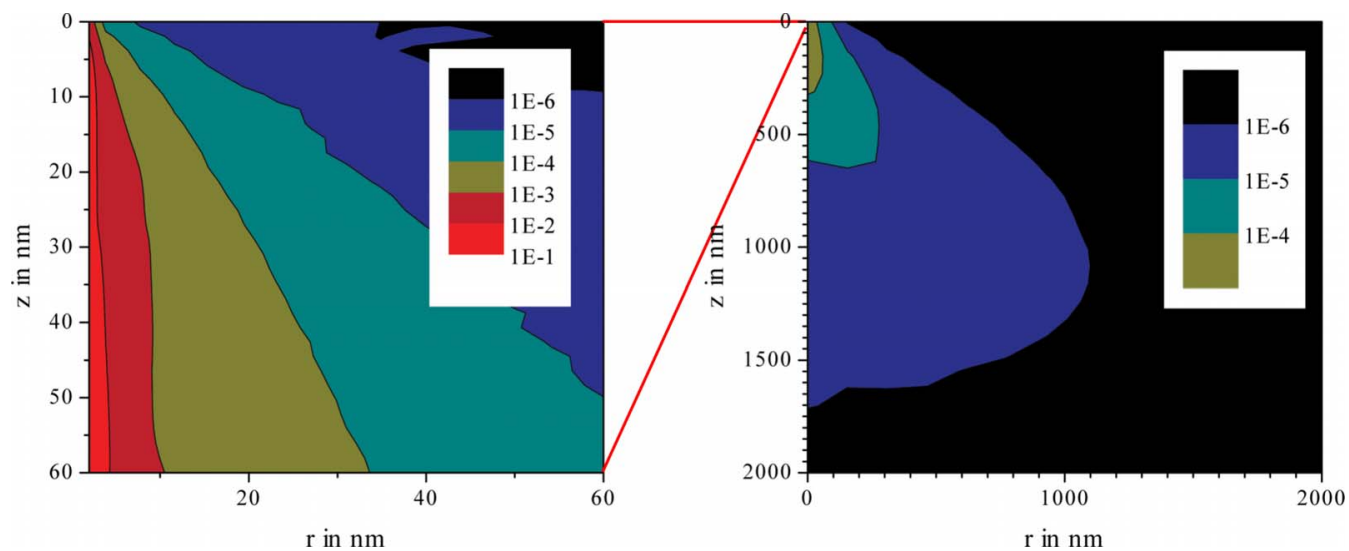

FIG. D1. MC simulation (Ref. 81) of the deposited energy density distribution in units of eV/nm ${ }^{3} / \mathrm{PE}$ into a Si bulk substrate by a $20 \mathrm{keV}$ electron beam with zero tilt and diameter. The electron beam incidence is at $r=z=0$. The left hand side of the figure represents a zoom into the vicinity of the incident electron beam. 


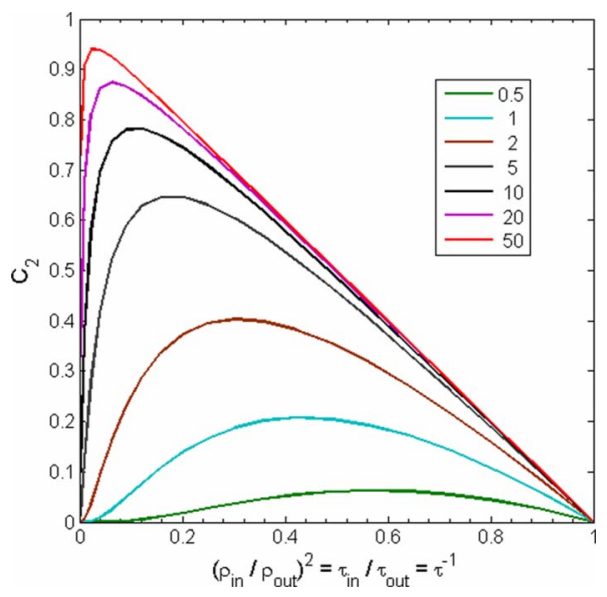

FIG. E1. Plot of $C_{2}$ vs the inverse of irradiative depletion $\left(\widetilde{\tau}^{-1}\right)$ according to Eq. (E1). The legend shows values of the normalized diffusion path outside the irradiated area: $2 \rho_{\text {out }} / \mathrm{FWHM}_{B}$.

tion center, respectively (see Sec. III A). The dependence $C_{2}\left(\widetilde{\tau}, \widetilde{\rho}_{\text {out }}\right)$ is shown in Fig. E1. The maximum of the diffusion contribution $C_{2}$ increases with mobility of the molecules (longer diffusion path).

The diffusion contribution tends to zero for very high depletions $\left(\widetilde{\tau}^{-1} \rightarrow 0\right)$ since the molecules are dissociated and fixed at very high rate leaving no time for diffusion. It also tends to zero at zero depletion $\left(\tilde{\tau}^{-1} \rightarrow 1\right)$ since there is no concentration gradient between the irradiated area and the surrounding. Furthermore, the diffusion contribution stays weak when the average diffusion path of the molecules on the surface is low compared to the beam size.

${ }^{1}$ D. J. Ehrlich et al., J. Vac. Sci. Technol. B 6, 895 (1988).

${ }^{2}$ R. L. Stewart, Phys. Rev. 45, 0488 (1934).

${ }^{3}$ K. M. Poole, Proc. Phys. Soc. London, Sect. B 66, 542 (1953).

${ }^{4}$ A. E. Ennos, Br. J. Appl. Phys. 5, 27 (1954).

${ }^{5}$ H. G. Heide, Lab. Invest. 14, 1134 (1965).

${ }^{6} \mathrm{H}$. G. Heide, Lab. Invest. 14, 1140 (1965).

${ }^{7}$ A. N. Broers et al., Appl. Phys. Lett. 29, 596 (1976).

${ }^{8}$ R. W. Christy, J. Appl. Phys. 31, 1680 (1960).

${ }^{9}$ S. Matsui and K. Mori, Jpn. J. Appl. Phys., Part 2 23, L706 (1984).

${ }^{10}$ S. Matsui and K. Mori, J. Vac. Sci. Technol. B 4, 299 (1986).

${ }^{11}$ H. W. P. Koops et al., J. Vac. Sci. Technol. B 6, 477 (1988).

${ }^{12}$ R. L. Seliger and W. P. Fleming, J. Vac. Sci. Technol. 10, 1127 (1973).

${ }^{13}$ J. H. Orloff and L. W. Swanson, J. Vac. Sci. Technol. 12, 1209 (1975).

${ }^{14}$ R. Levi-Setti, Scanning Electron Microscopy Part I, Proceedings of the Seventh Annual Scanning Electron Microscopy Symposium, IIT Research Institute, Chicago, Illinois 1974 (unpublished), p. 125.

${ }^{15}$ R. Clampitt, K. L. Aitken, and D. K. Jefferies, J. Vac. Sci. Technol. 12, 1208 (1975).

${ }^{16}$ R. Clampitt and D. K. Jefferies, Nucl. Instrum. Methods 149, 739 (1978).

${ }^{17}$ T. Liang and A. Stivers, Proc. SPIE 4688, 375 (2002).

${ }^{18}$ K. Edinger et al., J. Vac. Sci. Technol. B 22, 2902 (2004).

${ }^{19}$ S. J. Randolph, J. D. Fowlkes, and P. D. Rack, Crit. Rev. Solid State Mater. Sci. 31, 55 (2006).

${ }^{20}$ N. Silvis-Cividjian and C. W. Hagen, in Electron-Beam-Induced Nanometer-Scale Deposition Advances in Imaging and Electron Physics vol. 143, (Elsevier Academic, San Diego, 2006).

${ }^{21}$ M. D. Uchic et al., MRS Bull. 32, 408 (2007).

${ }^{22}$ M. A. Volkert and A. M. Minor, MRS Bull. 32, 389 (2007).

${ }^{23}$ R. M. Langford et al., MRS Bull. 32, 417 (2007).

${ }^{24}$ J. Mayer et al., MRS Bull. 32, 400 (2007).

${ }^{25}$ W. J. MoberlyChan et al., MRS Bull. 32, 424 (2007).
${ }^{26}$ S. Dhara, Crit. Rev. Solid State Mater. Sci. 32, 1 (2007).

${ }^{27}$ S. Matsui and R. Kometani, IEICE Trans. Electron. E90C, 25 (2007).

${ }^{28}$ P. M. Nellen, V. Callegari, and U. Sennhauser, Chimia 60, A735 (2006).

${ }^{29}$ A. A. Tseng, Small 1, 924 (2005).

${ }^{30}$ J. Gierak et al., Microelectron. Eng. 78-79, 266 (2005).

${ }^{31}$ S. Reyntjens and R. Puers, J. Micromech. Microeng. 11, 287 (2001).

${ }^{32}$ J. Melngailis, J. Vac. Sci. Technol. B 5, 469 (1987).

${ }^{33}$ L. A. Giannuzzi and F. A. Stevie, Introduction to Focused Ion Beams: Instrumentation, Theory, Techniques and Practice (Springer, New York, 2005).

${ }^{34}$ J. H. Orloff, M. Utlaut, and L. W. Swanson, High Resolution Focused Ion Beams (Kluwer, New York, 2003).

${ }^{35}$ L. Frey, C. Lehrer, and H. Ryssel, Appl. Phys. A: Mater. Sci. Process. 76, 1017 (2003).

${ }^{36}$ J. Y. Igaki et al., Microelectron. Eng. 83, 1225 (2006).

${ }^{37}$ E. Miyauchi et al., J. Vac. Sci. Technol. B 4, 189 (1986).

${ }^{38}$ C. Wilbertz et al., Nucl. Instrum. Methods Phys. Res. B 63, 120 (1992).

${ }^{39}$ R. Borret et al., J. Phys. D: Appl. Phys. 21, 1835 (1988).

${ }^{40}$ B. W. Ward, J. A. Notte, and N. P. Economou, J. Vac. Sci. Technol. B 24, 2871 (2006).

${ }^{41}$ L. Scipioni (private communication).

${ }^{42}$ L. Scipioni et al., J. Vac. Sci. Technol. B 18, 3194 (2000).

${ }^{43}$ K. Yamazaki and H. Namatsu, Jpn. J. Appl. Phys., Part 1 43, 3767 (2004).

${ }^{44}$ S. A. Rishton, S. P. Beaumont, and C. D. W. Wilkinson, J. Phys. E 17, 296 (1984).

${ }^{45}$ L. Reimer, Scanning Electron Microscopy: Physics of Image Formation and Microanalysis, 2nd ed. (Springer, Berlin, 1998).

${ }^{46}$ S. Babin et al., J. Vac. Sci. Technol. B 24, 2956 (2006).

${ }^{47}$ S. Babin et al., Abstracts of EIPBN'07, Denver, 2007 (unpublished), Paper No. 21.

${ }^{48}$ D. C. Joy, J. Microsc. 208, 24 (2002).

${ }^{49}$ K. D. Cummings et al., Appl. Phys. Lett. 48, 659 (1986).

${ }^{50}$ S. Shukuri et al., J. Electrochem. Soc. 134, 1536 (1987).

${ }^{51}$ J. W. Ward, R. L. Kubena, and M. W. Utlaut, J. Vac. Sci. Technol. B 6, 2090 (1988).

${ }^{52}$ V. Callegari and P. M. Nellen, Phys. Status Solidi A 204, 1665 (2007).

${ }^{53}$ J. Goldstein et al., Scanning Electron Microscopy and X-ray Microanalysis, 3rd ed. (Springer, New York, 2003), p. 586.

${ }^{54}$ J. J. L. Mulders (personal communication).

${ }^{55}$ J. Gierak et al., Microelectron. Eng. 84, 779 (2007).

${ }^{56}$ T. E. Allen, R. R. Kunz, and T. M. Mayer, J. Vac. Sci. Technol. B 6, 2057 (1988).

${ }^{57}$ A. D. Dubner et al., J. Appl. Phys. 70, 665 (1991).

${ }^{58}$ P. G. Blauner et al., J. Vac. Sci. Technol. B 7, 609 (1989).

${ }^{59}$ K. Edinger and T. Kraus, J. Vac. Sci. Technol. B 18, 3190 (2000).

${ }^{60}$ S. Lipp et al., Microelectron. Reliab. 36, 1779 (1996).

${ }^{61}$ N. Silvis-Cividjian et al., Microelectron. Reliab. 61-62, 693 (2002).

${ }^{62}$ R. R. Kunz and T. M. Mayer, J. Vac. Sci. Technol. B 5, 427 (1986).

${ }^{63}$ M. Fischer et al., Microelectron. Eng. 83, 784 (2006).

${ }^{64}$ P. C. Hoyle, J. R. A. Cleaver, and H. Ahmed, Appl. Phys. Lett. 64, 1448 (1994).

${ }^{65}$ P. C. Hoyle, J. R. A. Cleaver, and H. Ahmed, J. Vac. Sci. Technol. B 14, 662 (1996).

${ }^{66}$ J. D. Fowlkes, S. J. Randolph, and P. D. Rack, J. Vac. Sci. Technol. B 23, 2825 (2005).

${ }^{67}$ J. S. Ro, C. V. Thompson, and J. Melngailis, J. Vac. Sci. Technol. B 12, 73 (1994).

${ }^{68}$ G. Carter and J. S. Colligon, Ion Bombardment of Solids (Elsevier, Amsterdam, 1968), p. 41.

${ }^{69}$ Z. Xu, K. Gamo, and S. Namba, J. Vac. Sci. Technol. B 6, 1039 (1988).

${ }^{70}$ J. Schaefer and J. Hoelzl, Thin Solid Films 13, 81 (1972).

${ }^{71}$ M. S. Chung and T. E. Everhart, J. Appl. Phys. 45, 707 (1974).

${ }^{72}$ H. Farhang, E. Napchan, and B. H. Blott, J. Phys. D 26, 2266 (1993).

${ }^{73}$ J. F. Ziegler, http://www.srim.org/

${ }^{74}$ R. Kollath, in Handbuch der Physik, edited by S. Flügge (Springer, Berlin, 1956), pp. 232-303.

${ }^{75}$ Z. J. Ding, X. D. Tang, and R. Shimizu, J. Appl. Phys. 89, 718 (2001).

${ }^{76}$ Y. Mizuhara et al., J. Appl. Phys. 92, 6128 (2002).

${ }^{77}$ J. Cazaux, J. Appl. Phys. 59, 1418 (1986).

${ }^{78}$ D. C. Joy, http://www.mc-set.com/bse/index.htm

${ }^{79}$ K. S. Sim and J. D. White, J. Microsc. 217, 235 (2005).

${ }^{80}$ J. P. Biersack and L. G. Haggmark, Nucl. Instrum. Methods 174, 257 
(1980).

${ }^{81}$ L. Reimer, M. Kassens, and L. Wiese, Mikrochim. Acta, Suppl. 13, 485 (1996).

${ }^{82}$ M. Gryzinski, Phys. Rev. 138, A336 (1965).

${ }^{83}$ M. Gryzinski, Phys. Rev. 138, A305 (1965).

${ }^{84}$ M. Gryzinski, Phys. Rev. 138, A322 (1965).

${ }^{85}$ C. Moller, Ann. Phys. 14, 531 (1932).

${ }^{86}$ D. C. Joy, J. Microsc. 147, 51 (1987).

${ }^{87}$ D. C. Joy, Monte Carlo Modeling for Electron Microscopy and Microanalysis (Oxford University Press, New York, 1995).

${ }^{88}$ H. C. Petzold and P. J. Heard, J. Vac. Sci. Technol. B 9, 2664 (1991).

${ }^{89} \mathrm{~K}$. Edinger and T. Kraus, Microelectron. Eng. 57-58, 263 (2001).

${ }^{90}$ V. Friedli et al., Appl. Phys. Lett. 90, 053106 (2007).

${ }^{91}$ I. Utke et al., Appl. Phys. Lett. 88, 031906 (2006).

${ }^{92}$ W. F. van Dorp et al., Nano Lett. 5, 1303 (2005).

${ }^{93}$ L. G. Christophorou and J. K. Olthoff, Fundamental Electron Interactions with Plasma Processing Gases, 1st ed. (Kluwer Academic, New York/ Plenum, New York, 2004).

${ }^{94}$ B. C. Ibanescu et al., Phys. Chem. Chem. Phys. 9, 3163 (2007).

${ }^{95}$ T. Nakano and H. Sugai, Jpn. J. Appl. Phys., Part 1 31, 2919 (1992).

${ }^{96}$ Y.-K. Kim, K. K. Irikura et al., http://physics.nist.gov/PhysRefData/ Ionization/index. html

${ }^{97}$ D. A. Alman, D. N. Ruzic, and J. N. Brooks, Phys. Plasmas 7, 1421 (2000).

${ }^{98}$ R. E. Winters and R. W. Kiser, J. Phys. Chem. 69, 1618 (1965).

${ }^{99}$ R. E. Winters and R. W. Kiser, Inorg. Chem. 3, 699 (1964).

${ }^{100}$ R. E. Winters and R. W. Kiser, J. Organomet. Chem. 4, 190 (1965).

${ }^{101}$ R. E. Winters and R. W. Kiser, J. Phys. Chem. 69, 3198 (1965).

${ }^{102}$ R. E. Winters and R. W. Kiser, Inorg. Chem. 4, 157 (1965).

${ }^{103}$ R. W. Kiser, M. A. Krassoi, and R. J. Clark, J. Am. Chem. Soc. 89, 3653 (1967).

${ }^{104}$ I. Utke et al., Microelectron. Eng. 73-74, 553 (2004).

${ }^{105}$ P. Seuret et al., Phys. Chem. Chem. Phys. 5, 268 (2003).

${ }^{106}$ R. Balog et al., Int. J. Mass. Spectrom. 233, 267 (2004).

${ }^{107}$ D. Menzel, Surf. Interface Anal. 38, 1702 (2006).

${ }^{108}$ A. D. Bass and L. Sanche, Low Temp. Phys. 29, 202 (2003).

${ }^{109}$ P. Swiderek et al., J. Micro/Nanolith. MEMS MOEMS 111, 303 (2007).

${ }^{110}$ D. Kunze, O. Peters, and G. Sauerbrey, Z. Angew. Math. Phys. 22, 69 (1967).

${ }^{111}$ S. Mezhenny et al., J. Appl. Phys. 85, 3368 (1999).

${ }^{112}$ F. Y. C. Hui, G. Eres, and D. C. Joy, Appl. Phys. Lett. 72, 341 (1998).

${ }^{113}$ R. D. Ramsier, M. A. Henderson, and J. T. Yates, Surf. Sci. 257, 9 (1991).

${ }^{114}$ T. E. Madey, Science 234, 316 (1986).

${ }^{115}$ J. W. Coburn, Surf. Sci. 11, 61 (1968)

${ }^{116}$ M. L. Knotek and P. J. Feibelman, Phys. Rev. Lett. 40, 964 (1978).

${ }^{117}$ P. J. Feibelman and M. L. Knotek, Phys. Rev. B 18, 6531 (1978).

${ }^{118}$ D. Menzel and R. Gomer, J. Chem. Phys. 41, 3311 (1964).

${ }^{119}$ P. A. Redhead, Can. J. Phys. 42, 886 (1964).

${ }^{120}$ A. Muray et al., J. Vac. Sci. Technol. B 3, 367 (1985).

${ }^{121}$ A. Muray, M. Isaacson, and I. Adesida, Appl. Phys. Lett. 45, 589 (1984).

${ }^{122}$ J. A. Kelber and M. L. Knotek, Phys. Rev. B 30, 400 (1984).

${ }^{123}$ J. Cazaux, Microsc. Microanal. Microstruct. 6, 345 (1995).

${ }^{124}$ C. J. Humphreys et al., Scanning Microsc. Suppl. 4, 185 (1990).

${ }^{125}$ T. E. Madey, Surf. Sci. 300, 824 (1994).

${ }^{126}$ P. A. Redhead, Vacuum 48, 585 (1997).

${ }^{127}$ R. F. Egerton, P. Li, and M. Malac, Micron 35, 399 (2004).

${ }^{128}$ R. F. Egerton and C. J. Rossouw, J. Phys. D 9, 659 (1976).

${ }^{129}$ X. W. Du et al., Appl. Phys. Lett. 82, 1108 (2003).

${ }^{130}$ A. Kis et al., Nat. Mater. 3, 153 (2004).

${ }^{131}$ I. Jang et al., Nano Lett. 4, 109 (2004).

${ }^{132}$ S. Frabboni, G. C. Gazzadi, and A. Spessot, Appl. Phys. Lett. 89, 113108 (2006).

${ }^{133}$ M. P. Seah et al., Surf. Interface Anal. 37, 444 (2005).

${ }^{134}$ Y. Yamamura and H. Tawara, At. Data Nucl. Data Tables 62, 149 (1996).

${ }^{135}$ H. E. Roosendaal, in Sputtering by Particle Bombardment. Physical Sputtering of Single Element Solids (Springer, New York, 1981), Vol. 1, p. 145.

${ }^{136}$ X. Xu et al., J. Vac. Sci. Technol. B 10, 2675 (1992).

${ }^{137}$ T. J. Stark et al., J. Vac. Sci. Technol. B 13, 2565 (1995).

${ }^{138}$ J. B. Wang, A. Datta, and Y. L. Wang, Appl. Surf. Sci. 135, 129 (1998).

${ }^{139}$ K. P. Mueller and H. C. Petzold, Proc. SPIE 1263, 12 (1990).

${ }^{140}$ D. Santamore et al., J. Vac. Sci. Technol. B 15, 2346 (1997).
${ }^{141}$ A. Lugstein et al., Appl. Phys. A: Mater. Sci. Process. 76, 545 (2003).

${ }^{142}$ H. B. Kim et al., Nanotechnology 18, 265307 (2007).

${ }^{143}$ H. B. Kim et al., Nanotechnology 18, 245303 (2007).

${ }^{144}$ A. Lugstein et al., Nucl. Instrum. Methods Phys. Res. B 255, 309 (2007).

${ }^{145}$ A. Datta et al., Nanotechnology 16, 2764 (2005).

${ }^{146}$ C. Schoendorfer et al., J. Appl. Phys. 102, 044308 (2007).

${ }^{147} \mathrm{G}$. Dearnale et al., Can. J. Phys. 46, 587 (1968).

${ }^{148} \mathrm{~A}$. Lugstein et al. (unpublished).

${ }^{149}$ J. D. Casey et al., J. Vac. Sci. Technol. B 20, 2682 (2002).

${ }^{150}$ H. H. Anderson and H. L. Bay, in Sputtering by Particle Bombardment. Physical Sputtering of Single Element Solids (Springer, New York, 1981), Vol. 1, p. 145.

${ }^{151}$ H. Yamaguchi, J. Phys. Colloq. 48, 65 (1987).

${ }^{152}$ J. G. Pellerin et al., J. Vac. Sci. Technol. B 7, 1810 (1989).

${ }^{153}$ D. P. Adams et al., J. Vac. Sci. Technol. B 21, 2334 (2003).

${ }^{154}$ A. Stanishevsky, Thin Solid Films 398, 560 (2001).

${ }^{155}$ A. J. Steckl and I. Chyr, J. Vac. Sci. Technol. B 17, 362 (1999).

${ }^{156} \mathrm{~J}$. R. Riordon and J. Melngailis (unpublished).

${ }^{157}$ J. Melngailis et al., J. Vac. Sci. Technol. B 4, 176 (1986).

${ }^{158}$ J. J. L. Mulders, D. A. M. de Winter, and W. J. H. C. P. Duinkerken, Microelectron. Eng. 84, 1540 (2007).

${ }^{159}$ V. Ignatova et al., Prog. Surf. Sci. 81, 247 (2006).

${ }^{160}$ M. P. Seah, Nucl. Instrum. Methods Phys. Res. B 229, 348 (2005).

${ }^{161}$ M. P. Seah, Nucl. Instrum. Methods Phys. Res. B 239, 286 (2005).

${ }^{162}$ S. Agostinelli et al., Nucl. Instrum. Methods Phys. Res. A 506, 250 (2003).

${ }^{163}$ Ionshaper,http://www.ims.co.at/products_services_ionshaper.html

${ }^{164}$ D. P. Adams and M. J. Vasile, J. Vac. Sci. Technol. B 24, 836 (2006).

${ }^{165}$ D. P. Adams, M. J. Vasile, and T. M. Mayer, J. Vac. Sci. Technol. B 24, 1766 (2006).

${ }^{166}$ M. A. Henderson, R. D. Ramsier, and J. T. Yates, J. Vac. Sci. Technol. A 9, 2785 (1991).

${ }^{167}$ B. B. Dayton, in Foundations of Vacuum Science and Technology, edited by J. M. Lafferty (Wiley, New York, 1998).

${ }^{168}$ T. T. Kodas and M. J. Hampden-Smith, The Chemistry of Metal CVD (VCH, Weinheim, 1994).

${ }^{169}$ T. Ohta et al., Chem. Vap. Deposition 7, 33 (2001).

${ }^{170}$ R. Chellappa and D. Chandra, J. Chem. Thermodynamics 37, 377 (2005).

${ }^{171}$ T. Tao et al., J. Vac. Sci. Technol. B 8, 1826 (1990).

${ }^{172}$ K. K. Choi and S. W. Rhee, Thin Solid Films 409, 147 (2002).

${ }^{173}$ W. Wagner and A. Pruss, J. Phys. Chem. Ref. Data 22, 783 (1993).

${ }^{174}$ M. G. M. Vandervis and E. H. P. Cordfunke, Thermochim. Acta 265, 129 (1995).

${ }^{175}$ SigmaAldrich, http://www.sigmaaldrich.com/

${ }^{176}$ A. Berman, Vacuum Engineering Calculations, Formulas, and Solved Exercises (Academic, San Diego, 1992).

${ }^{177}$ M. Knudsen, Ann. Phys. 28, 75 (1909).

${ }^{178}$ R. J. Cole, Rarefied Gas Dyn. 10, 261 (1976)

${ }^{179}$ I. Utke et al., Microelectron. Eng. 83, 1499 (2006).

${ }^{180}$ M. S. El-Morsi et al., Proc. SPIE 5567, 467 (2004).

${ }^{181} \mathrm{~V}$. Friedli et al., Abstracts of EIPBN'06, Baltimore 2006 (unpublished).

${ }^{182}$ T. Bret, I. Utke, and P. Hoffmann, Microelectron. Eng. 78-79, 307 (2005).

${ }^{183}$ X. Tang and D. C. Joy, Scanning 27, 293 (2005).

${ }^{184}$ C. J. Chen, J. Vac. Sci. Technol. A 5, 3386 (1987).

${ }^{185}$ P. A. Crozier et al., Appl. Phys. Lett. 84, 3441 (2004).

${ }^{186}$ M. Tanaka et al., Appl. Phys. A: Mater. Sci. Process. 78, 543 (2004).

${ }^{187}$ V. Scheuer, H. Koops, and T. Tschudi, Microelectron. Eng. 5, 423 (1986),

${ }^{188}$ A. D. Dubner and A. Wagner, J. Appl. Phys. 66, 870 (1989).

${ }^{189}$ J. Y. Tsao, H. J. Zeiger, and D. J. Ehrlich, Surf. Sci. 160, 419 (1985).

${ }^{190}$ T. Bret et al., Microelectron. Eng. 78-79, 300 (2005).

${ }^{191}$ Y. S. Chung et al., Surf. Sci. 482, 312 (2001).

${ }^{192}$ S. Brunauer, P. H. Emmett, and E. Teller, J. Am. Chem. Soc. 60, 309 (1938).

${ }^{193}$ E. G. Seebauer and C. E. Allen, Prog. Surf. Sci. 49, 265 (1995).

${ }^{194}$ M. U. Kislyuk, React. Kinet. Catal. Lett. 39, 229 (1998).

${ }^{195}$ D. W. Bjarneson and N. O. Petersen, J. Am. Chem. Soc. 112, 988 (1990).

${ }^{196}$ X. Tang and D. C. Joy, Scanning 26, 226 (2004).

${ }^{197}$ F. Banhart, Phys. Rev. E 52, 5156 (1995).

${ }^{198} \mathrm{~F}$. Banhart, http://www.staff.uni-mainz.de/banhart/c-nanostructures/ filaments.htm

${ }^{199}$ I. Lyubinetsky et al., J. Appl. Phys. 86, 4949 (1999).

${ }^{200}$ I. Lyubinetsky et al., J. Vac. Sci. Technol. A 17, 1445 (1999). 
${ }^{201}$ I. Lyubinetsky et al., Surf. Sci. 459, L451 (2000).

${ }^{202}$ A. D. Kent et al., Science 262, 1249 (1993).

${ }^{203}$ J. Cazaux, Ultramicroscopy 60, 411 (1995).

${ }^{204}$ I. Utke et al., Adv. Eng. Mater. 7, 323 (2005).

${ }^{205}$ P. Kern et al., Appl. Phys. Lett. 89, 02102 (2006).

${ }^{206}$ T. Ishitani and H. Kaga, J. Electron Microsc. 44, 331 (1995).

${ }^{207}$ D. Chu et al., Proc. SPIE 4689, 206 (2002).

${ }^{208}$ D. C. Chu et al., J. Vac. Sci. Technol. B 20, 3044 (2002).

${ }^{209}$ D. C. Chu et al., J. Vac. Sci. Technol. B 21, 2985 (2003).

${ }^{210}$ S. J. Randolph, J. D. Fowlkes, and P. D. Rack, J. Appl. Phys. 97, 124312 (2005).

${ }^{211}$ M. Weber, J. Phys. D: Appl. Phys. 27, 1363 (1994).

${ }^{212}$ I. Utke et al., Appl. Phys. Lett. 81, 3245 (2002).

${ }^{213}$ P. Buffat and J. P. Borel, Phys. Rev. A 13, 2287 (1976)

${ }^{214}$ P. A. Buffat, Philos. Trans. R. Soc. London, Ser. A 361, 291 (2003).

${ }^{215}$ W. Zhang et al., Adv. Eng. Mater. 8, 711 (2006).

${ }^{216}$ W. Zhang et al., J. Mater. Sci. 41, 2577 (2006).

${ }^{217}$ M. Rueb, H. W. P. Koops, and T. Tschudi, Microelectron. Eng. 9, 251 (1989).

${ }^{218}$ I. Utke et al., J. Vac. Sci. Technol. B 25, 2219 (2007).

${ }^{219}$ K. H. Mueller, Optik (Jena) 33, 296 (1971).

${ }^{220}$ P. Hirsch et al., Scanning 16, 101 (1994).

${ }^{221}$ L. Reimer and M. Wachter, Ultramicroscopy 3, 169 (1978).

${ }^{222}$ V. V. Aristov, N. A. Kislov, and I. I. Hodos, Izv. Akad. Nauk. SSSR, Fiz. Zemli 55, 1523 (1991).

${ }^{223}$ N. A. Kislov, Scanning 15, 212 (1993).

${ }^{224}$ I. Haller and P. White, J. Phys. Chem. 67, 1784 (1963).

${ }^{225}$ S. Haraichi and M. Komuro, Jpn. J. Appl. Phys., Part 1326168 (1993).

${ }^{226}$ D. Lichtman, in Surface Science: Recent Progress and Perspectives, edited by T. S. Jayadevaiah and R. Vanselow (CRC, Cleveland, 1974), pp. 104-135.

${ }^{227}$ W. Li and D. C. Joy, J. Vac. Sci. Technol. A 24, 431 (2006).

${ }^{228}$ C. R. Fritzsche, J. Appl. Phys. 60, 2182 (1986).

${ }^{229}$ F. G. Rudenauer, W. Steiger, and D. Schrottmayer, J. Vac. Sci. Technol. B 6, 1542 (1988).

${ }^{230}$ L. R. Harriott, J. Vac. Sci. Technol. B 11, 2012 (1993).

${ }^{231}$ S. Lipp et al., J. Vac. Sci. Technol. B 143920 (1996).

${ }^{232}$ C. Santschi et al., Nanotechnology 17, 2722 (2006).

${ }^{233}$ P. C. Hoyle et al., Appl. Phys. Lett. 62, 3043 (1993).

${ }^{234}$ M. Toth et al., J. Appl. Phys. 101, 054309 (2007).

${ }^{235}$ C. J. Lobo et al., Nanotechnology 19, 025303 (2008).

${ }^{236}$ T. P. Chiang, H. H. Sawin, and C. V. Thompson, J. Vac. Sci. Technol. A 15, 3104 (1997).

${ }^{237}$ C. W. Hagen, N. Silvis-Cividjian and P. Kruit, Scanning 28, 204 (2006).

${ }^{238}$ N. Silvis-Cividjian, C. W. Hagen, and P. Kruit, J. Appl. Phys. 98, 084905 (2005).

${ }^{239}$ K. I. Schiffmann, Nanotechnology 4, 163 (1993).

${ }^{240}$ A. Luisier et al., J. Electrochem. Soc. 151, C535 (2004).

${ }^{241}$ A. Y. Kasumov et al., Mikroelektronika 23, 287 (1994).

${ }^{242}$ N. Silvis-Cividjian et al., Appl. Phys. Lett. 82, 3514 (2003).

${ }^{243}$ O. Guise et al., Appl. Phys. Lett. 85, 2352 (2004).

${ }^{244}$ M. Tanaka et al., Surf. Interface Anal. 37, 261 (2005).

${ }^{245}$ M. Shimojo et al., J. Microsc. 214, 76 (2004).

${ }^{246}$ M. Shimojo et al., Appl. Surf. Sci. 241, 56 (2005).

${ }^{247}$ K. Mitsuishi et al., Appl. Phys. Lett. 83, 2064 (2003).

${ }^{248}$ Y. Ochiai et al., Jpn. J. Appl. Phys., Part 1 30, 3266 (1991).

${ }^{249}$ W. F. van Dorp et al., Microelectron. Eng. 83, 1468 (2006).

${ }^{250}$ W. F. van Dorp et al., J. Vac. Sci. Technol. B 24, 618 (2006)

${ }^{251}$ D. A. Smith, J. D. Fowlkes, and P. D. Rack, Nanotechnology 18, 265308 (2007).

${ }^{252}$ K. Rykaczewski, W. B. White, and A. G. Fedorov, J. Appl. Phys. 101, 054307 (2007).

${ }^{253}$ W. F. van Dorp and C. W. Hagen, J. Appl. Phys. (submitted).

${ }^{254}$ K. T. Kohlmann-von Platen et al., J. Vac. Sci. Technol. B 11, 2219 (1993).

${ }^{255}$ W. H. Brunger and K. T. Kohlmann, J. Microelectromech. Syst. 2, 30 (1993).

${ }^{256}$ B. Hubner, H. W. P. Koops, H. Pagnia et al., Ultramicroscopy 42, 1519 (1992).

${ }^{257}$ U. Hubner et al., Microelectron. Eng. 57-58, 953 (2001).

${ }^{258}$ T. Bret et al., Appl. Phys. Lett. 83, 4005 (2003).

${ }^{259}$ H. W. P. Koops et al., Jpn. J. Appl. Phys., Part 1 33, 7099 (1994).

${ }^{260}$ R. Sharma et al., Microsc. Microanal. 9, 912 (2003).
${ }^{261}$ S. Ketharanathan et al., J. Vac. Sci. Technol. B 24, 678 (2006).

${ }^{262}$ H. G. Heide, Z. Angew. Phys. 15, 116 (1963).

${ }^{263}$ R. Dobrozemsky, S. Menhart, and K. Buchtela, J. Vac. Sci. Technol. A 25, 551 (2007)

${ }^{264}$ R. L. Jackson and M. R. Trusheim, J. Am. Chem. Soc. 104, 6590 (1982).

${ }^{265}$ M. W. Grinstaff et al., Ultrasonics 30, 168 (1992).

${ }^{266}$ B. D. Fahlman and A. R. Barron, Adv. Mater. Opt. Electron. 10, 223 (2000).

${ }^{267}$ J. S. Chickos and W. E. Acree, J. Phys. Chem. Ref. Data 31, 537 (2002).

${ }^{268}$ J. S. Chickos and W. E. Acree, J. Phys. Chem. Ref. Data 32, 519 (2003).

${ }^{269}$ T. Bret, Ph.D. thesis, EPFL, 2005.

${ }^{270}$ H. Adachi, H. Nakane, and M. Katamoto, Appl. Surf. Sci. 76, 11 (1994).

${ }^{271}$ S. Kiyohara, H. Takamatsu, and K. Mori, Semicond. Sci. Technol. 17, 1096 (2002).

${ }^{272}$ Y. Ochiai et al., Jpn. J. Appl. Phys., Part 1 32, 6147 (1993).

${ }^{273}$ M. J. Vasile and L. R. Harriott, J. Vac. Sci. Technol. B 7, 1954 (1989).

${ }^{274}$ O. Guise et al., MRS Symposia Proceedings No. 803 (Materials Research Society, Pittsburgh, 2004), pp. 3-8.

${ }^{275}$ O. Guise et al., Surf. Sci. 571, 128 (2004).

${ }^{276}$ I. Jungwirthova et al., Rev. Sci. Instrum. 73, 3302 (2002).

${ }^{277}$ S. Matsui, Nucl. Instrum. Methods Phys. Res. B 257, 758 (2007).

${ }^{278}$ L. R. Thompson et al., Appl. Phys. Lett. 43, 777 (1983).

${ }^{279}$ F. Hirose and H. Sakamoto, Jpn. J. Appl. Phys., Part 1 34, 5904 (1995).

${ }^{280}$ K. Nakano, T. Horie, and H. Sakamoto, Jpn. J. Appl. Phys., Part 1 35, 6570 (1996).

${ }^{281}$ F. Bozso and P. Avouris, Phys. Rev. B 38, 3943 (1988).

${ }^{282}$ L. Torrison et al., Thin Solid Films 434, 106 (2003).

${ }^{283}$ J. McMurran et al., Inorg. Chem. 37, 6638 (1998).

${ }^{284}$ J. McMurran et al., J. Am. Chem. Soc. 120, 5233 (1998).

${ }^{285}$ R. J. Hamers, Surf. Sci. 583, 1 (2005).

${ }^{286}$ Y. Pauleau, Thin Solid Films 122, 243 (1984).

${ }^{287}$ S. Matsui, T. Ichihashi, and M. Mito, J. Vac. Sci. Technol. B 7, 1182 (1989).

${ }^{288}$ M. Komuro and H. Hiroshima, J. Vac. Sci. Technol. B 15, 2809 (1997).

${ }^{289}$ M. Komuro and H. Hiroshima, Microelectron. Eng. 35, 273 (1997).

${ }^{290} \mathrm{H}$. Hiroshima and M. Komuro, Jpn. J. Appl. Phys., Part 1 36, 7686 (1997).

${ }^{291}$ H. Hiroshima and M. Komuro, Nanotechnology 9, 108 (1998).

${ }^{292}$ H. Hiroshima et al., Jpn. J. Appl. Phys., Part 1 38, 7135 (1999).

${ }^{293}$ R. B. Jackman and J. S. Foord, Appl. Phys. Lett. 49, 196 (1986)

${ }^{294}$ D. A. Bell et al., J. Vac. Sci. Technol. B 12, 2976 (1994).

${ }^{295}$ H. O. Funsten, J. W. Boring, and R. E. Johnson, J. Appl. Phys. 71, 1475 (1992).

${ }^{296}$ S. H. Kim and G. A. Somorjai, J. Phys. Chem. B 106, 1386 (2002).

${ }^{297}$ P. Hoffmann (unpublished).

${ }^{298}$ A. Landsberg and C. L. Hoatson, J. Less-Common Met. 22, 327 (1970).

${ }^{299}$ T. Ichihashi and S. Matsui, J. Vac. Sci. Technol. B 6, 1869 (1988).

${ }^{300}$ S. Matsui and M. Mito, Appl. Phys. Lett. 53, 1492 (1988).

${ }^{301}$ Z. Xu et al., J. Vac. Sci. Technol. B 7, 1959 (1989).

${ }^{302}$ K. Gamo and S. Namba, MRS Symposia Proceedings No. 131 (Materials Research Society, Pittsburgh, 1989), p. 531.

${ }^{303}$ S. Wang, Y. M. Sun, and J. M. White, Appl. Surf. Sci. 249, 110 (2005).

${ }^{304}$ H. H. Madden, J. Vac. Sci. Technol. 13, 228 (1976).

${ }^{305}$ D. R. Sandstrom, J. H. Leck, and E. E. Donaldson, J. Appl. Phys. 38, 2851 (1967).

${ }^{306}$ P. A. Redhead, Appl. Phys. Lett. 4, 166 (1964).

${ }^{307}$ M. L. Shek, S. P. Withrow, and W. H. Weinberg, Surf. Sci. 72, 678 (1978).

${ }^{308}$ R. R. Kunz and T. M. Mayer, Appl. Phys. Lett. 50, 962 (1987).

${ }^{309}$ R. R. Kunz and T. M. Mayer, J. Vac. Sci. Technol. B 5, 427 (1987).

${ }^{310}$ R. R. Kunz and T. M. Mayer, J. Vac. Sci. Technol. B 6, 1557 (1988).

${ }^{311}$ K. Mitsuishi et al., Jpn. J. Appl. Phys., Part 1 44, 5627 (2005).

${ }^{312}$ M. Takeguchi, M. Shimojo, and K. Furuya, Jpn. J. Appl. Phys., Part 1 44, 5631 (2005).

${ }^{313}$ M. Tanaka et al., J. Mater. Sci. 41, 2667 (2006).

${ }^{314}$ M. Tanaka et al., Jpn. J. Appl. Phys., Part 1 44, 5635 (2005).

${ }^{315}$ T. Lukasczyk et al., Small 6, 841 (2008).

${ }^{316}$ M. A. Bruk et al., High Energy Chem. 39, 65 (2005)

${ }^{317}$ P. Hoffmann et al., Appl. Surf. Sci. 43, 54 (1989).

${ }^{318}$ P. Hoffmann et al., J. Appl. Phys. 74, 7588 (1993).

${ }^{319}$ P. Hoffmann et al., J. Vac. Sci. Technol. B 9, 3483 (1991).

${ }^{320}$ T. Tanabe et al., Vib. Spectrosc. 18, 141 (1998).

${ }^{321}$ I. Utke et al., Appl. Phys. Lett. 80, 4792 (2002). 
${ }^{322}$ I. Utke et al., MRS Symposia Proceedings No. 706 (Materials Research Society, Pittsburgh, 2002), pp. 307-312.

${ }^{323}$ G. Boero et al., Appl. Phys. Lett. 86, 042503 (2005).

${ }^{324}$ Y. M. Lau et al., J. Vac. Sci. Technol. A 20, 1295 (2002).

${ }^{325}$ H. Papp, Surf. Sci. 149, 460 (1985).

${ }^{326}$ K. A. Prior, K. Schwaha, and R. M. Lambert, Surf. Sci. 77, 193 (1978).

${ }^{327}$ U. Bardi, P. Tiscione, and G. Rovida, Appl. Surf. Sci. 27, 299 (1986).

${ }^{328}$ M. E. Bridge, C. M. Comrie, and R. M. Lambert, Surf. Sci. 67, 393 (1977).

${ }^{329}$ T. Kruck, Angew. Chem. 6, 53 (1967).

${ }^{330}$ A. Perentes et al., J. Vac. Sci. Technol. B 25, 2228 (2007).

${ }^{331}$ S. Wang et al., J. Vac. Sci. Technol. B 22, 1803 (2004).

${ }^{332}$ J. D. Barry et al., J. Vac. Sci. Technol. B 24, 3165 (2006).

${ }^{333}$ I. Utke et al., J. Vac. Sci. Technol. B 18, 3168 (2000).

${ }^{334}$ P. Hoffmann et al., MRS Symposia Proceedings No. 624 (Materials Research Society, Pittsburgh, 2000), pp. 171-177.

${ }^{335} \mathrm{~F}$. Cicoira et al., J. Cryst. Growth 265, 619 (2004).

${ }^{336}$ T. Bret et al., Microelectron. Eng. 83, 1482 (2006).

${ }^{337}$ J. C. Hierso et al., Appl. Organomet. Chem. 12, 161 (1998).

${ }^{338}$ R. R. Kunz, T. E. Allen, and T. M. Mayer, J. Vac. Sci. Technol. B 5, 1427 (1987).

${ }^{339}$ M. Shimojo et al., Appl. Phys. A: Mater. Sci. Process. 79, 1869 (2004).

${ }^{340} \mathrm{~K}$. Mitsuishi et al., Ultramicroscopy 103, 17 (2005).

${ }^{341}$ M. Tanaka et al., Appl. Phys. Lett. 86, 183104 (2005).

${ }^{342}$ N. A. Kislov et al., Scanning 18, 114 (1996).

${ }^{343}$ M. Weber et al., J. Vac. Sci. Technol. B 13, 1364 (1995).

${ }^{344}$ S. Bauerdick et al., J. Vac. Sci. Technol. B 24, 3144 (2006).

${ }^{345}$ D. K. Stewart, L. A. Stern, and J. C. Morgan, Proc. SPIE 1089, 18 (1989).

${ }^{346}$ I. J. Luxmoore et al., Thin Solid Films 515, 6791 (2007).

${ }^{347}$ F. Cicoira et al., Appl. Surf. Sci. 242, 107 (2005).

${ }^{348}$ G. Wilkinson, J. Am. Chem. Soc. 73, 5501 (1951).

${ }^{349}$ T. Brintlinger et al., J. Vac. Sci. Technol. B 23, 3174 (2005).

${ }^{350}$ M. E. Gross, L. R. Harriott, and R. L. Opila, J. Appl. Phys. 68, 4820 (1990).

${ }^{351}$ I. I. Grinvald et al., Bull. Acad. Sci. USSR, Phys. Ser. (Engl. Transl.) 37, 2068 (1988)

${ }^{352}$ A. Perentes and P. Hoffmann, Chem. Vap. Deposition 13, 176 (2007).

${ }^{353}$ A. Perentes, P. Hoffmann, and F. Munnik, Proc. SPIE 6533, 65331Q (2007).

${ }^{354}$ A. Ishibashi, K. Funato, and Y. Mori, J. Vac. Sci. Technol. B 9, 169 (1991).

${ }^{355}$ H. O. Funsten, Nucl. Instrum. Methods Phys. Res. B 72, 183 (1992).

${ }^{356}$ E. O. Fischer and W. Hafner, Z. Naturforsch. B 10, 665 (1955).

${ }^{357}$ K. W. Choi et al., J. Phys. Chem. A 108, 11292 (2004).

${ }^{358}$ J. G. Clabes et al., U.S. Patent No. 5,171,992, (1992).

${ }^{359}$ W. W. Pai et al., J. Vac. Sci. Technol. B 15, 785 (1997).

${ }^{360}$ Z. Xue et al., Chem. Mater. 4, 162 (1992).

${ }^{361}$ V. N. Emel'yanenko et al., J. Chem. Thermodyn. 39, 594 (2007).

${ }^{362}$ M. Ishikawa et al., Jpn. J. Appl. Phys., Part 1 43, 1833 (2004).

${ }^{363}$ L. Rotkina, J. F. Lin, and J. P. Bird, Appl. Phys. Lett. 83, 4426 (2003)

${ }^{364}$ O. Yavas et al., Appl. Phys. Lett. 76, 3319 (2000).

${ }^{365}$ O. Yavas et al., J. Vac. Sci. Technol. B 18, 976 (2000).

${ }^{366}$ J. Puretz and L. W. Swanson, J. Vac. Sci. Technol. B 10, 2695 (1992).

${ }^{367}$ T. Tao, W. Wilkinson, and J. Melngailis, J. Vac. Sci. Technol. B 9, 162 (1991).

${ }^{368}$ R. M. Langford, T. X. Wang, and D. Ozkaya, Microelectron. Eng. 84, 784 (2007).

${ }^{369}$ M. A. V. R. Dasilva and M. L. C. C. H. Ferrao, Pure Appl. Chem. 60, 1225 (1988).

${ }^{370}$ P. G. Blauner et al., J. Vac. Sci. Technol. B 7, 1816 (1989).

${ }^{371}$ I. Utke et al., Microelectron. Eng. 53, 261 (2000).

${ }^{372}$ K. L. Lee and M. Hatzakis, J. Vac. Sci. Technol. B 7, 941 (1989).

${ }^{373}$ H. W. P. Koops et al., J. Vac. Sci. Technol. B 11, 2386 (1993).

${ }^{374}$ S. Ketharanathan, R. Sharma, and J. Drucker, J. Vac. Sci. Technol. B 23, 2403 (2005)

${ }^{375}$ Y. Ochiai, J. Fujita, and S. Matsui, J. Vac. Sci. Technol. B 14, 3887 (1996).

${ }^{376}$ A. D. Dellaratta, J. Melngailis, and C. V. Thompson, J. Vac. Sci. Technol. B 11, 2195 (1993).

${ }^{377}$ T. J. Gannon et al., J. Vac. Sci. Technol. B 22, 3000 (2004).

${ }^{378}$ H. W. P. Koops et al., Microelectron. Eng. 30, 539 (1996).

${ }^{379}$ A. Perentes et al., J. Vac. Sci. Technol. B 24, 587 (2006).
${ }^{380}$ R. J. Young and J. Puretz, J. Vac. Sci. Technol. B 13, 2576 (1995).

${ }^{381}$ A. N. Campbell et al., Proceedings of the 23rd International Symposium for Testing and Failure Analysis, Santa Clara, CA, 1997 (unpublished), p. 223.

${ }^{382}$ K. Edinger, J. Melngailis, and J. Orloff, J. Vac. Sci. Technol. B 16, 3311 (1998).

${ }^{383}$ Kenji Gamo et al., Microelectron. Eng. 5, 163 (1986).

${ }^{384} \mathrm{P}$. Hoffmann et al., Proc. SPIE 5925, 1 (2005).

${ }^{385}$ A. Botman et al., Nanotechnology 17, 3779 (2006).

${ }^{386}$ A. Folch et al., J. Vac. Sci. Technol. B 14, 2609 (1996).

${ }^{387}$ A. Folch et al., Appl. Phys. Lett. 66, 2080 (1995).

${ }^{388}$ K. Molhave et al., Nano Lett. 3, 1499 (2003).

${ }^{389}$ T. P. Chiang, H. H. Sawin, and C. V. Thompson, J. Vac. Sci. Technol. A 15, 2677 (1997).

${ }^{390}$ M. Takeguchi et al., J. Mater. Sci. 41, 4532 (2006)

${ }^{391}$ M. Shimojo, M. Takeguchi, and K. Furuya, Nanotechnology 17, 3637 (2006).

${ }^{392}$ T. Nagata et al., Appl. Phys. Lett. 87, 013103 (2005).

${ }^{393}$ A. Perentes and P. Hoffman, J. Vac. Sci. Technol. B 25, 2233 (2007).

${ }^{394}$ H. Komano, Y. Ogawa, and T. Takigawa, Jpn. J. Appl. Phys., Part 128 , 2372 (1989).

${ }^{395}$ Y. Talmon et al., J. Microsc. 117, 321 (1979).

${ }^{396}$ J. Taniguchi et al., Jpn. J. Appl. Phys., Part 1 36, 7691 (1997).

${ }^{397}$ J. Niitsuma et al., Jpn. J. Appl. Phys., Part 2 45, L71 (2006).

${ }^{398}$ T. Bret et al., U.S. Patent No. 2,006,228,634 (2005).

${ }^{399}$ J. W. Coburn and H. F. Winters, J. Appl. Phys. 50, 3189 (1979).

${ }^{400}$ S. J. Randolph, J. D. Fowlkes, and P. D. Rack, J. Appl. Phys. 98, 034902 (2005).

${ }^{401}$ H. P. Gillis, J. L. Clemons, and J. P. Chamberlain, J. Vac. Sci. Technol. B 10, 2729 (1992).

${ }^{402}$ H. P. Gillis et al., MRS Internet J. Nitride Semicond. Res. 4, (1999).

${ }^{403}$ S. Wang et al., J. Vac. Sci. Technol. B 23, 206 (2005).

${ }^{404}$ Y. M. Sun et al., Appl. Surf. Sci. 252, 311 (2005).

${ }^{405}$ Y. Ochiai et al., J. Vac. Sci. Technol. B 5, 423 (1987).

${ }^{406}$ M. Komuro, N. Watanabe, and H. Hiroshima, Jpn. J. Appl. Phys., Part 1 29, 2288 (1990).

${ }^{407}$ R. J. Young, J. R. A. Cleaver, and H. Ahmed, J. Vac. Sci. Technol. B 11, 234 (1993).

${ }^{408}$ Y. K. Park et al., Jpn. J. Appl. Phys., Part 1 36, 7712 (1997).

${ }^{409}$ T. Kosugi et al., J. Vac. Sci. Technol. B 9, 2660 (1991).

${ }^{410}$ V. Callegari et al., J. Vac. Sci. Technol. B 25, 2175 (2007).

${ }^{411}$ V. Callegari et al., Appl. Surf. Sci. 253, 8969 (2007).

${ }^{412}$ H. Nakamura, H. Komano, and M. Ogasawara, Jpn. J. Appl. Phys., Part 1 31, 4465 (1992).

${ }^{413}$ R. R. Kola, G. K. Celler, and L. R. Harriott, MRS Symposia Proceedings No. 279 (Materials Research Society, Pittsburgh, 1993), pp. 593-598.

${ }^{414}$ J. D. Casey et al., Microelectron. Eng. 24, 43 (1994).

${ }^{415}$ K. Edinger, J. Vac. Sci. Technol. B 17, 3058 (1999).

${ }^{416}$ X. L. Fu et al., Microelectron. Eng. 78-79, 29 (2005).

${ }^{417}$ K. Gamo et al., J. Vac. Sci. Technol. B 6, 989 (1988).

${ }^{418}$ A. Stanishevsky et al., J. Vac. Sci. Technol. B 21, 3067 (2003).

${ }^{419}$ G. Schmid, Angew. Chem., Int. Ed. 47, 3496 (2008).

${ }^{420}$ B. F. G. Johnson et al., Chem. Commun. 14, 1317 (2000).

${ }^{421}$ H. G. Craighead and L. M. Schiavone, Appl. Phys. Lett. 48, 1748 (1986).

${ }^{422}$ L. R. Harriott et al., Appl. Phys. Lett. 49, 1661 (1986).

${ }^{423}$ P. D. Rack, J. D. Fowlkes, and S. J. Randolph, Nanotechnology 18, 465602 (2007).

${ }^{424}$ T. Bret et al., J. Vac. Sci. Technol. B 22, 2504 (2004).

${ }^{425}$ N. Antoniou et al., J. Vac. Sci. Technol. B 20, 2695 (2002).

${ }^{426}$ V. Friedli et al., in Applied Scanning Probe Methods VIII, edited by B. Bushan, H. Fuchs, and M. Tomitori (Springer, Berlin, 2008).

${ }^{427}$ M. Prestigiacomo et al., Microelectron. Eng. 76, 175 (2004).

${ }^{428}$ R. Kometani, Proceedings of the MNE, Copenhague, 2007 (unpublished).

${ }^{429}$ W. F. van Dorp et al., J. Vac. Sci. Technol. B 25, 2210 (2007).

${ }^{430}$ J. S. Ro, C. V. Thompson, and J. Melngailis, Thin Solid Films 258, 333 (1995).

${ }^{431}$ J. Fujita et al., J. Vac. Sci. Technol. B 19, 2834 (2001).

${ }^{432}$ M. Ishida et al., J. Vac. Sci. Technol. B 21, 2728 (2003).

${ }^{433}$ I. Utke et al., Adv. Eng. Mater. 8, 155 (2006).

${ }^{434}$ S. Bauerdick et al., Microelectron. Eng. 67-68, 963 (2003).

${ }^{435}$ T. Liang et al., J. Vac. Sci. Technol. B 18, 3216 (2000).

${ }^{436}$ T. Liang et al., J. Vac. Sci. Technol. B 23, 3101 (2005).

${ }^{437}$ P. D. Prewett et al., J. Vac. Sci. Technol. B 11, 2427 (1993). 
${ }^{438}$ Nawotec, http://www.smt.zeiss.com/nawotec.

${ }^{439}$ H. D. Wanzenboeck et al., J. Vac. Sci. Technol. B 24, 2755 (2006).

${ }^{440}$ T. Liang et al., Proc. SPIE 5567, 456 (2004).

${ }^{441}$ K. Edinger et al., Proc. SPIE 5130, 383 (2003).

${ }^{442}$ W. Jianhua et al., Semicond. Sci. Technol. 18, 199 (2003).

${ }^{443}$ L. Folks et al., Appl. Phys. Lett. 76, 909 (2000).

${ }^{444}$ Y. Akama et al., J. Vac. Sci. Technol. A 8, 429 (1990).

${ }^{445}$ Nanotools, http://www.nano-tools.com/

${ }^{446} \mathrm{G}$. Janchen et al., Appl. Phys. Lett. 80, 4623 (2002).

${ }^{447}$ M. Wendel, H. Lorenz, and J. P. Kotthaus, Appl. Phys. Lett. 67, 3732 (1995).

${ }^{448}$ Y. R. Choi et al., Scanning 28, 311 (2006).

${ }^{449}$ S. Matsui and T. Ichihashi, Appl. Phys. Lett. 53, 842 (1988)

${ }^{450}$ Z. Q. Liu, K. Mitsuishi, and K. Furuya, J. Appl. Phys. 96, 3983 (2004).

${ }^{451}$ J. Fujita et al., J. Vac. Sci. Technol. B 21, 2990 (2003).

${ }^{452}$ V. V. Ristov, A. Y. Kasumov, N. A. Kislov et al., Nanotechnology 6, 35 (1995).

${ }^{453}$ J. H. Kindt et al., Nanotechnology 15, 1131 (2004).

${ }^{454}$ M. Takeguchi et al., J. Mater. Sci. 41, 2627 (2006).

${ }^{455}$ M. Takeguchi, M. Shimojo, and K. Furuya, Nanotechnology 16, 1321 (2005).

${ }^{456}$ O. Sqalli et al., J. Appl. Phys. 92, 1078 (2002).

${ }^{457}$ M. Castagne et al., Ultramicroscopy 76, 187 (1999).

${ }^{458}$ I. W. Rangelow et al., Microelectron. Eng. 57-58, 737 (2001).

${ }^{459}$ K. Edinger, T. Gotszalk, and I. W. Rangelow, J. Vac. Sci. Technol. B 19, 2856 (2001).

${ }^{460}$ E. J. Sanchez, J. T. Krug, and X. S. Xie, Rev. Sci. Instrum. 73, 3901 (2002).

${ }^{461}$ S. Matsui et al., in Science and Technology of Mesoscopic Structures, edited by S. Namba, C. Hamaguchi, and T. Ando (Springer, Tokyo, 1992).

${ }^{462}$ D. Litvinov and S. Khizroev, Nanotechnology 13, 179 (2002).

${ }^{463}$ S. K. Khizroev et al., IEEE Trans. Magn. 35, 2544 (1999).

${ }^{464}$ T. Koshikawa et al., IEEE Trans. Magn. 34, 1471 (1998).

${ }^{465}$ M. R. Koblischka, U. Hartmann, and T. Sulzbach, Mater. Sci. Eng., C 23, 747 (2003).

${ }^{466}$ A. Meister et al., Appl. Phys. Lett. 85, 6260 (2004).

${ }^{467}$ Y. B. Koh, K. Gamo, and S. Namba, J. Vac. Sci. Technol. B 9, 2648 (1991).

${ }^{468}$ H. W. P. Koops et al., Microelectron. Eng. 57-58, 995 (2001).

${ }^{469}$ H. J. Lezec et al., Science 297, 820 (2002).

${ }^{470}$ A. Perentes et al., Microelectron. Eng. 73-74, 412 (2004).

${ }^{471}$ S. Graells et al., Appl. Phys. Lett. 91, 121112 (2007).

${ }^{472}$ T. W. Ebbesen et al., Nature (London) 391, 667 (1998)

${ }^{473}$ P. M. Nellen and R. Bronnimann, Meas. Sci. Technol. 17, 943 (2006).

${ }^{474}$ P. M. Nellen, V. Callegari, and R. Bronnimann, Microelectron. Eng. 83, 1805 (2006)

${ }^{475}$ M. Roussey et al., Appl. Phys. Lett. 87, 241101 (2005)

${ }^{476}$ W. Brezna et al., Appl. Phys. Lett. 88, 122116 (2006).

${ }^{477}$ J. J. Senkevich and S. B. Desu, Chem. Vap. Deposition 4, 92 (1998).

${ }^{478}$ N. Miura et al., Jpn. J. Appl. Phys., Part 2 36, 1619 (1997).

${ }^{479}$ N. Miura et al., Appl. Surf. Sci. 113-114, 269 (1997).

${ }^{480}$ H. W. P. Koops, A. Kaya, and M. Weber, J. Vac. Sci. Technol. B 13, 2400 (1995).

${ }^{481}$ H. W. P. Koops et al., J. Vac. Sci. Technol. B 14, 4105 (1996).

${ }^{482}$ F. Arai et al., 2003 Third IEEE Conference on Nanotechnology: IEEENANO 20032003 (unpublished), pp. 811-814.

${ }^{483}$ Y. Tsukatani et al., Jpn. J. Appl. Phys., Part 1 44, 5683 (2005).

${ }^{484}$ P. G. Li, A. Z. Jin, and W. H. Tang, Phys. Status Solidi A 203, 282 (2006).

${ }^{485}$ Y. K. Park et al., Nucl. Instrum. Methods Phys. Res. B 148, 25 (1999).

${ }^{486}$ V. Gopal et al., Appl. Phys. Lett. 85, 49 (2004).

${ }^{487}$ K. Molhave et al., Nanotechnology 15, 1047 (2004).

${ }^{488}$ R. M. Langford et al., J. Vac. Sci. Technol. B 24, 2306 (2006).

${ }^{489}$ A. Romano-Rodriguez and F. Hernandez-Ramirez, Microelectron. Eng. 84, 789 (2007)

${ }^{490}$ V. Gopal et al., Nano Lett. 4, 2059 (2004).

${ }^{491}$ D. Tham, C. Y. Nam, and J. E. Fischer, Adv. Mater. (Weinheim, Ger.) 18, 290 (2006).

${ }^{492}$ A. Motayed et al., J. Appl. Phys. 100, 024306 (2006).

${ }^{493}$ M. Prestigiacomo et al., Appl. Phys. Lett. 86, 192112 (2005)

${ }^{494}$ T. Morita et al., J. Vac. Sci. Technol. B 21, 2737 (2003).

${ }^{495}$ M. Komuro, H. Hiroshima, and A. Takechi, Nanotechnology 9, 104 (1998).
${ }^{496}$ G. C. Gazzadi and S. Frabboni, J. Vac. Sci. Technol. B 23, L1 (2005).

${ }^{497}$ A. Candini et al., J. Magn. Magn. Mater. 310, 2752 (2007).

${ }^{498}$ C. H. Chen et al., J. Vac. Sci. Technol. B 16, 2898 (1998).

${ }^{499}$ S. Kohmoto et al., J. Vac. Sci. Technol. B 12, 3699 (1994).

${ }^{500}$ T. Takahashi et al., Appl. Phys. Lett. 60, 68 (1992).

${ }^{501}$ A. V. Krasheninnikov and F. Banhart, Nat. Mater. 6, 723 (2007).

${ }^{502}$ K. Maehashi et al., Appl. Phys. Lett. 90, 023103 (2007).

${ }^{503}$ S. Bauerdick et al., J. Vac. Sci. Technol. B 22, 3539 (2004).

${ }^{504}$ R. Kometani et al., Jpn. J. Appl. Phys., Part 2 45, L711 (2006).

${ }^{505}$ C. Schoessler and H. W. P. Koops, J. Vac. Sci. Technol. B 16, 862 (1998),

${ }^{506}$ C. Schossler, J. Urban, and H. W. P. Koops, J. Vac. Sci. Technol. B 15, 1535 (1997).

${ }^{507}$ C. Schossler et al., Microelectron. Eng. 30, 471 (1996).

${ }^{508}$ K. Murakami and M. Takai, J. Vac. Sci. Technol. B 22, 1266 (2004).

${ }^{509}$ C. Lehrer et al., J. Vac. Sci. Technol. B 22, 1402 (2004).

${ }^{510}$ W. Jarupoonphol et al., J. Vac. Sci. Technol. B 21, 1598 (2003).

${ }^{511}$ C. Ochiai et al., J. Vac. Sci. Technol. B 19, 933 (2001).

${ }^{512}$ C. Ochiai et al., IDW'00, Proceedings of the Seventh International Display Workshops, 2000 (unpublished), pp. 967-970.

${ }^{513}$ M. Takai et al., Microelectron. Eng. 41-42, 453 (1998).

${ }^{514}$ M. Takai et al., IVMC'96, Ninth International Vacuum Microelectronics Conference, 1996 (unpublished), p. 657.

${ }^{515}$ H. Morimoto et al., Jpn. J. Appl. Phys., Part 1 35, 6623 (1996).

${ }^{516}$ J. Kretz et al., Microelectron. Eng. 23, 477 (1994).

${ }^{517}$ H. W. P. Koops et al., Nucl. Instrum. Methods Phys. Res. A 363, 1 (1995).

${ }^{518}$ H. W. P. Koops, E. Dobisz, and J. Urban, J. Vac. Sci. Technol. B 15, 1369 (1997).

${ }^{519}$ M. Weber, et al., J. Vac. Sci. Technol. B 13, 461 (1995).

${ }^{520}$ H. W. P. Koops and C. Schossler, IVMC'96, Ninth International Vacuum Microelectronics Conference, 1996 (unpublished), pp. 458-462.

${ }^{521}$ F. Floreani, H. W. Koops, and W. Elsasser, Microelectron. Eng. 57-58, 1009 (2001).

${ }^{522}$ X. Yang et al., Appl. Phys. Lett. 86, 183106 (2005).

${ }^{523}$ F. Floreani, H. W. Koops, and W. Elsasser, Nucl. Instrum. Methods Phys. Res. A 483, 488 (2002).

${ }^{524}$ K. Murakami, F. Wakaya, and M. Takai, J. Vac. Sci. Technol. B 25, 1310 (2007).

${ }^{525}$ J. Sellmair, K. Edinger, and H. W. P. Koops, J. Vac. Sci. Technol. B 23, 781 (2005)

${ }^{526}$ K. Murakami et al., J. Vac. Sci. Technol. B 23, 759 (2005).

${ }^{527}$ J. Brugger et al., Microelectron. Eng. 35, 401 (1997).

${ }^{528}$ I. Sieber et al., Electrochem. Solid-State Lett. 6, C1 (2003).

${ }^{529}$ T. Djenizian, L. Santinacci, and P. Schmuki, J. Electrochem. Soc. 151, G175 (2004).

${ }^{530}$ T. Djenizian, J. Macak, and P. Schmuki, MRS Symposia Proceedings No. 741 (Materials Research Society, Pittsburgh, 2003), pp. 79-83.

${ }^{531}$ T. Djenizian et al., Nanotechnology 17, 5363 (2006).

${ }^{532}$ W. J. Mitchell and E. L. Hu, Appl. Phys. Lett. 74, 1916 (1999).

${ }^{533}$ Y. Hiratani et al., Jpn. J. Appl. Phys., Part 2 29, L1360 (1990).

${ }^{534}$ M. Taneya et al., Jpn. J. Appl. Phys., Part 2 28, L515 (1989).

${ }^{535}$ K. Akita et al., Semicond. Sci. Technol. 6, 699 (1991).

${ }^{536}$ A. J. Steckl, H. C. Mogul, and S. Mogren, Appl. Phys. Lett. 60, 1833 (1992).

${ }^{537}$ K. Watanabe et al., J. Vac. Sci. Technol. B 22, 22 (2004).

${ }^{538}$ T. Morita et al., Jpn. J. Appl. Phys., Part 1 42, 3874 (2003).

${ }^{539}$ M. N. Lin et al., Appl. Phys. Lett. 87, 173116 (2005).

${ }^{540}$ L. X. Dong, F. Arai, and T. Fukuda, Appl. Phys. Lett. 81, 1919 (2002).

${ }^{541}$ P. A. Williams et al., Appl. Phys. Lett. 80, 2574 (2002).

${ }^{542}$ M.-F. Yu et al., Science 287, 637 (2000).

${ }^{543}$ W. Ding et al., J. Appl. Phys. 98, 014905 (2005).

${ }^{544}$ S. Hoffmann et al., Nanotechnology 18, 205503 (2007).

${ }^{545}$ S. Orso et al., Adv. Mater. (Weinheim, Ger.) 18, 874 (2006).

${ }^{546}$ M. Becker et al., Nano Lett. 7, 75 (2007).

${ }^{547}$ T. D. Yuzvinsky et al., Appl. Phys. Lett. 86, 053109 (2005).

${ }^{548}$ P. Boggild et al., Nanotechnology 12, 331 (2001).

${ }^{549}$ R. Kometani et al., J. Vac. Sci. Technol. B 23, 298 (2005).

${ }^{550}$ J. Y. Igaki et al., Microelectron. Eng. 83, 1221 (2006).

${ }^{551}$ T. Morita et al., J. Vac. Sci. Technol. B 22, 3137 (2004).

${ }^{552}$ W. Q. Ding et al., Compos. Sci. Technol. 66, 1112 (2006).

${ }^{553}$ S. Okada et al., Jpn. J. Appl. Phys., Part 1455556 (2006)

${ }^{554}$ J. Fujita et al., Nucl. Instrum. Methods Phys. Res. B 206, 472 (2003).

${ }^{555}$ J. Nilsson et al., Adv. Mater. (Weinheim, Ger.) 18, 427 (2006) 
${ }^{556}$ T. Hoshino et al., J. Vac. Sci. Technol. B 24, 2538 (2006).

${ }^{557}$ C. Danelon et al., Langmuir 22, 10711 (2006).

${ }^{558} \mathrm{~J}$. Li et al., Nature (London) 412166 (2001).

${ }^{559}$ H. Miyazoe et al., Appl. Phys. Lett. 92, 043124 (2008).

${ }^{560}$ R. Kometani et al., Microelectron. Eng. 83, 1642 (2006).

${ }^{561}$ W. F. van Dorp et al., Nanotechnology 19, 225305 (2008).

${ }^{562}$ B. Ward, J. A. Notte, and N. P. Economou, Photonics Spectra 41, 68 (2007).

${ }^{563}$ J. Notte and B. Ward, Scanning 28, 63 (2006).
${ }^{564}$ H. Loeschner et al., J. Vac. Sci. Technol. B 19, 2520 (2001).

${ }^{565}$ J. Melngailis et al., J. Vac. Sci. Technol. B 16, 927 (1998).

${ }^{566}$ E. Platzgummer, H. Loeschner, and G. Gross, Proc. SPIE 6730, 6730033 (2007).

${ }^{567}$ I. L. Berry et al., J. Vac. Sci. Technol. B 15, 2382 (1997).

${ }^{568}$ K. Kanaya and S. Okayama, J. Phys. D: Appl. Phys. 5, 43 (1972).

${ }^{569}$ H. J. Fitting, J. Electron Spectrosc. Relat. Phenom. 136, 265 (2004).

${ }^{570}$ J. F. Ziegler, Nucl. Instrum. Methods Phys. Res. B 219, 1027 (2004). 